\title{
Synthesis of Acyl Phosphoramidates Employing a Modified Staudinger Reaction
}

Iain Currie, Brad E. Sleebs

The Walter and Eliza Hall Institute of Medical Research, Parkville, Victoria, 3052, Australia.

Department of Medical Biology, The University of Melbourne, Parkville, Victoria, 3010 Australia.

Index

Page

S2 - S5 General methods

S6 - S27 Procedures

S28 References

S29 - S113 NMR spectra and LC chromatograms. 


\section{General Methods.}

Solvents were obtained commercially and used without further purification. $H$-Phosphonate starting materials are known compounds and were purchased from Sigma-Aldrich or CombiBlocks and used without further purification. Chromatography was performed using SiliCycle SiliaSep silica gel flash cartridges $60 \AA$ (particle size $40-63 \mu \mathrm{m}$ ) in combination with an automated purification system unless otherwise stated. Heating of reaction mixture was performed using a hotplate fitted with a DrySyn heating block. NMR spectra were recorded on a Bruker Avance DRX 300. Chemical shifts are reported in parts per million (ppm) and referenced to the appropriate solvent peak. $\mathrm{MeOD}, \mathrm{DMSO}-\mathrm{d}_{6}$, Acetone- $\mathrm{D}_{6}$ and $\mathrm{CDCl}_{3}$ contain $\mathrm{H}_{2} \mathrm{O} .{ }^{31} \mathrm{P}$ NMR spectra were referenced to $85 \% \mathrm{H}_{3} \mathrm{PO}_{4}$ as an external standard. LCMS analysis was performed using an Agilent LCMS system composed of an Agilent G6120B Mass Detector, 1260 Infinity G1312B Binary pump, 1260 Infinity G1367E HiPALS autosampler and 1260 Infinity G4212B Diode Array Detector, Poroshell 120 EC-C18, 2.1 x 50 mm $2.7 \mu \mathrm{m}$ column, injection volume $2 \mu \mathrm{L}$, gradient: 5 - 100\% B over $3 \min$ (solvent A: water $0.1 \%$ formic acid; solvent B: $\mathrm{MeCN}$ 0.1\% formic acid), flow rate: $0.8 \mathrm{~mL} / \mathrm{min}$, detection: $214 \mathrm{~nm}$, acquisition time: $5 \mathrm{~min}$. LCMS conditions used to assess purity of final compounds were as follows, column: Phenomenex Gemini C18, 2.0 x $50 \mathrm{~mm}$; injection volume $20 \mu \mathrm{L}$; gradient: 0-100\% Buffer B over 6 min (buffer A: 0.1\% formic acid in autoclaved MilliQ water; buffer B: $0.1 \%$ formic acid in $100 \%$ acetonitrile), flow rate: $1.0 \mathrm{~mL} / \mathrm{min}$, detection: 214 or $254 \mathrm{~nm}$. High resolution mass spectra were acquired through The Bio21 Mass Spectrometry and Proteomics Facility using a Thermo Scientific ${ }^{\mathrm{TM}}$ nano-LC Quadrupole Exactive ${ }^{\mathrm{TM}}$ Plus Orbitrap mass spectrometer. Acyl azides were prepared using standard procedures by reaction of acyl chlorides with sodium azide $(\mathbf{3 a}, \mathbf{g}, \mathbf{h}, \mathbf{m}, \mathbf{p}, \mathbf{q}, \mathbf{r}, \mathbf{s}, \mathbf{t})$ or via reaction of carboxylic acids with DPPA (3i, $\mathbf{j}, \mathbf{k}, \mathbf{l}, \mathbf{n})$. The acyl azides were isolated without purification and stored at -20 ${ }^{\circ} \mathrm{C}$.

CAUTION: Azido compounds may represent an explosion hazard when being concentrated under vacuum or stored neat. A safety shield and appropriate handling procedures are recommended. Additional safety precautions should be considered for reactions greater than $10 \mathrm{mmol} \mathrm{scale.}$ 


\section{General Procedures}

General procedure A - Synthesis of acyl phosphoramidates from acyl azide.

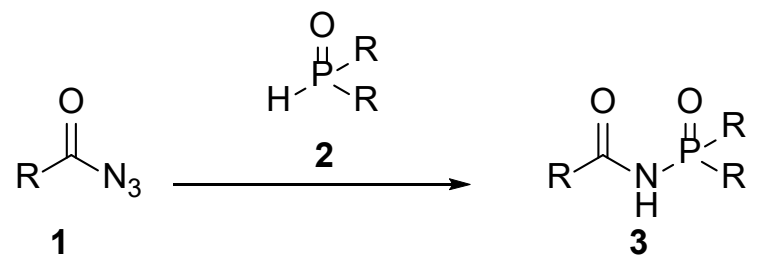

Trimethylsilyl chloride $(0.15 \mathrm{~mL}, 1.2 \mathrm{mmol})$ was added to a solution of the acyl azide $(1.0$ $\mathrm{mmol}), H$-phosphonate $(0.262 \mathrm{~g}, 1.0 \mathrm{mmol})$ and triethylamine $(0.21 \mathrm{~mL}, 1.5 \mathrm{mmol})$ in DCM $(10 \mathrm{~mL})$ and stirred for $18 \mathrm{~h}$ at room temperature. The reaction mixture was then purified by column chromatography eluting with a gradient from 100\% DCM to 5\% $\mathrm{MeOH} / \mathrm{DCM}$. In most of the examples, the purified product crystallized on standing or precipitation was induced by trituration with $\mathrm{Et}_{2} \mathrm{O}$.

General procedure B - Synthesis of imidodiphosphates from phosphoryl azide.

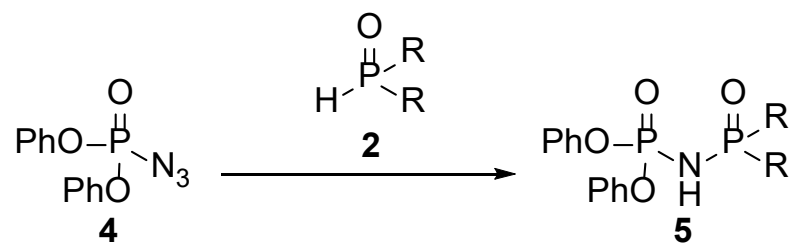

Trimethylsilyl chloride $(0.15 \mathrm{~mL}, 1.2 \mathrm{mmol})$ was added to a solution of DPPA $(0.215 \mathrm{~mL}, 1.0$ $\mathrm{mmol}), H$-phosphonate $(1.0 \mathrm{mmol})$ and triethylamine $(0.21 \mathrm{~mL}, 1.5 \mathrm{mmol})$ in DCM $(10 \mathrm{~mL})$. the reaction mixture was stirred for $18 \mathrm{~h}$ at room temperature. The reaction mixture was then washed with saturated $\mathrm{KHSO}_{4}(20 \mathrm{~mL})$, dried over $\mathrm{MgSO}_{4}$, filtered and then concentrated. The residue was then purified by column chromatography gradient eluting with $100 \%$ DCM to 5\% $\mathrm{MeOH} / \mathrm{DCM}$ to give $\mathbf{5}$. 


\section{General procedure C - Synthesis of acyl phosphoramidates from carboxylic acid.}

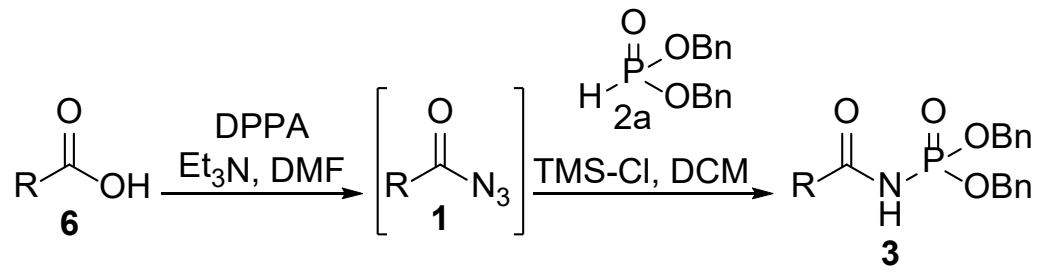

A solution of the carboxylic acid $(1.1 \mathrm{mmol})$ and triethylamine $(0.56 \mathrm{~mL}, 4.0 \mathrm{mmol})$ in DMF $(5 \mathrm{~mL})$ was cooled on ice and DPPA $(0.21 \mathrm{~mL}, 1.0 \mathrm{mmol})$ was added. The reaction mixture was stirred for $1 \mathrm{~h}$ at $0{ }^{\circ} \mathrm{C}$. The reaction mixture was then removed from the ice bath. After complete consumption of DPPA, the mixture was diluted with DCM $(20 \mathrm{~mL})$ and the $H$-phosphonate $(1.0 \mathrm{mmol})$ was added followed by trimethylsilyl chloride $(0.42 \mathrm{~mL}, 3.3$ mmol). The reaction mixture was stirred at room temperature for $18 \mathrm{~h}$. The reaction mixture was concentrated to dryness then diluted with EtOAc $(100 \mathrm{~mL})$ then washed successively with saturated $\mathrm{KHSO}_{4}(20 \mathrm{~mL})$, water $(2 \times 100 \mathrm{~mL})$, saturated $\mathrm{NaHCO}_{3}(20 \mathrm{~mL})$ and brine $(50 \mathrm{~mL})$. The organic layer was then dried over $\mathrm{MgSO}_{4}$, filtered and concentrated. The residue was then purified by column chromatography gradient eluting with $100 \%$ heptane to $100 \%$ EtOAc to give 3 .

\section{General procedure C - 10 mmol scale}<smiles>O=C(O)NC(Cc1ccccc1)C(=O)O</smiles>

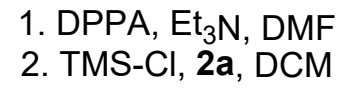<smiles>O=C(N[C@@H](Cc1ccccc1)C(=O)NP(=O)(OCc1ccccc1)OCc1ccccc1)c1ccccc1</smiles>

A solution of Boc-L-Phe-OH (2.918 g, $11.0 \mathrm{mmol})$ and triethylamine $(5.58 \mathrm{~mL}, 40.0 \mathrm{mmol})$ in DMF (20 mL) was cooled on ice and DPPA $(2.15 \mathrm{~mL}, 10.0 \mathrm{mmol})$ was added, and the reaction mixture stirred for $1 \mathrm{~h}$ at $0{ }^{\circ} \mathrm{C}$. The reaction mixture was then removed from the ice bath and after complete consumption of DPPA, the mixture was diluted with DCM (200 mL) and dibenzyl phosphite $(2.21 \mathrm{~mL}, 10.0 \mathrm{mmol})$ was added followed by trimethylsilyl chloride $(4.19$ $\mathrm{mL}, 33.0 \mathrm{mmol}$ ). The reaction mixture was stirred for $18 \mathrm{~h}$ at room temperature. The reaction mixture was then concentrated to dryness, diluted in EtOAc $(300 \mathrm{~mL})$ then washed successively with saturated $\mathrm{KHSO}_{4}(50 \mathrm{~mL})$, water $(2 \times 200 \mathrm{~mL})$, saturated $\mathrm{NaHCO}_{3}(2 \times 20$ 
$\mathrm{mL})$ and brine $(20 \mathrm{~mL})$. The organic layer was then dried over $\mathrm{MgSO}_{4}$, filtered and concentrated. The residue was then purified by column chromatography eluting with $100 \%$ heptane to $100 \%$ EtOAc to yield 3ab as a white solid (4.18 g, 80\%). ${ }^{1} \mathrm{H}$ NMR (300 MHz, $\left.\mathrm{CDCl}_{3}\right) \delta 8.44(\mathrm{~d}, J 10.9 \mathrm{~Hz}, 1 \mathrm{H}), 7.40-7.27(\mathrm{~m}, 10 \mathrm{H}), 7.24-7.13(\mathrm{~m}, 5 \mathrm{H}), 5.23-4.96(\mathrm{~m}$, $5 \mathrm{H}), 4.51-4.31(\mathrm{~m}, 1 \mathrm{H}), 3.07(\mathrm{dd}, J 14.0,5.6 \mathrm{~Hz}, 1 \mathrm{H}), 2.97-2.81(\mathrm{~m}, 1 \mathrm{H}), 1.36(\mathrm{~s}, 9 \mathrm{H}) ;{ }^{13} \mathrm{C}$ NMR (75 MHz, DMSO-d6) $\delta 174.4$ (d, J4.4 Hz), 155.6, 137.7, 136.1 (dd, J 8.0, 2.6 Hz), 129.3, 128.4, 128.2, 128.0, 127.9, 127.7, 126.3, 78.2, 68.3 - 67.7 (m), 56.8 (d, J 11.7 Hz), 36.3, 28.1; ${ }^{31} \mathrm{P}$ NMR (121 MHz, $\left.\mathrm{CDCl}_{3}\right) \delta-2.02$; HRMS (ESI) m/z: $[\mathrm{M}+\mathrm{H}]^{+}$Calcd for $\mathrm{C}_{28} \mathrm{H}_{34} \mathrm{~N}_{2} \mathrm{O} 66$ : 525.2155, found: 525.2152 .

Note: Additional aromatic signal in ${ }^{13} \mathrm{C}-\mathrm{NMR}$ spectrum in the range of $130-125 \mathrm{ppm}$. Attributed to the presence of a doublet which cannot be assigned unambiguously.

General procedure D - Synthesis of acyl phosphoramidate from hydrazide.

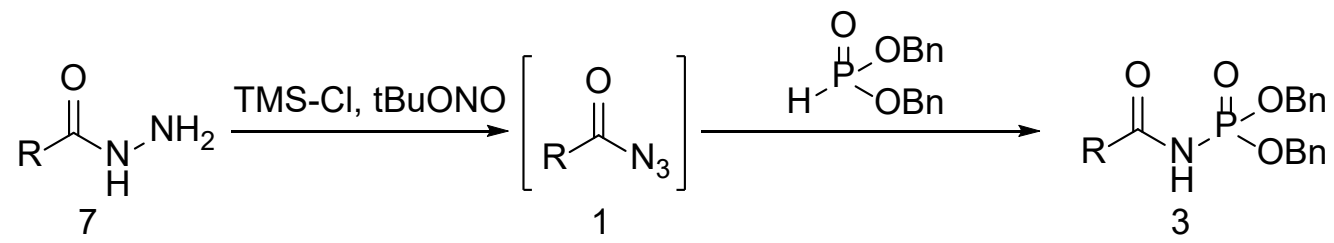

Trimethylsilyl chloride ( $3 \mathrm{mmol}$ ) was added to a solution of the hydrazide $(1.0 \mathrm{mmol})$ in DMF $(5 \mathrm{~mL})$ and the solution was cooled on ice. tert-Butyl nitrite $(1.0 \mathrm{mmol})$ was then added and after $1 \mathrm{~h}$, the reaction mixture was removed from the ice bath. The reaction mixture was then diluted with DCM (30 mL) and triethylamine (4 eq) was added, followed by the $H$-phosphonate $(1.0 \mathrm{mmol})$. The reaction mixture was then stirred for $18 \mathrm{~h}$ at room temperature. The reaction mixture was concentrated to dryness then diluted with EtOAc $(100 \mathrm{~mL})$ then washed successively with saturated $\mathrm{KHSO}_{4}(20 \mathrm{~mL})$, water $(2 \times 100 \mathrm{~mL})$, saturated $\mathrm{NaHCO}_{3}(20 \mathrm{~mL})$ and brine $(50 \mathrm{~mL})$. The organic layer was then dried over $\mathrm{MgSO}_{4}$, filtered and concentrated. The residue was then purified by column chromatography eluting with $100 \%$ heptane to $100 \%$ EtOAc to give $\mathbf{3}$. 


\section{Dibenzyl benzoylphosphoramidate (3a)}<smiles>O=C(NP(=O)(OCc1ccccc1)OCc1ccccc1)c1ccccc1</smiles>

General procedure A was followed. Trimethylsilyl chloride $(0.15 \mathrm{~mL}, 1.2 \mathrm{mmol})$ was added to a solution of benzoyl azide $(0.147 \mathrm{~g}, 1.0 \mathrm{mmol})$, dibenzyl phosphite $(0.262 \mathrm{~g}, 1.0 \mathrm{mmol})$ and triethylamine $(0.21 \mathrm{~mL}, 1.5 \mathrm{mmol})$ in DCM $(10 \mathrm{~mL})$, and the reaction mixture stirred for $18 \mathrm{~h}$ at room temperature. The reaction mixture was purified using column chromatography gradient eluting from $100 \% \mathrm{DCM}$ to $5 \% \mathrm{MeOH} / \mathrm{DCM}$ to yield $3 \mathrm{a}$ as a white solid $(0.335 \mathrm{~g}, 88 \%)$. ${ }^{1} \mathrm{H}$ NMR (300 MHz, $\left.\mathrm{CDCl}_{3}\right) \delta 9.02(\mathrm{~d}, J 9.4 \mathrm{~Hz}, 1 \mathrm{H}), 8.00-7.92(\mathrm{~m}, 2 \mathrm{H}), 7.60-7.50(\mathrm{~m}, 1 \mathrm{H})$, $7.46-7.24(\mathrm{~m}, 12 \mathrm{H}), 5.32-5.14(\mathrm{~m}, 4 \mathrm{H}) ;{ }^{13} \mathrm{C} \mathrm{NMR}\left(75 \mathrm{MHz}, \mathrm{CDCl}_{3}\right) \delta 168.0(\mathrm{~d}, J 2.6 \mathrm{~Hz})$, 135.8 (d, J 6.9 Hz), 132.9, 132.6 (d, $J 10.5 \mathrm{~Hz}), 128.7,128.6,128.4,128.1,69.8$ (d, J 5.7 Hz); ${ }^{31} \mathrm{P}$ NMR (121 MHz, $\left.\mathrm{CDCl}_{3}\right) \delta-0.71$; HRMS (ESI) m/z: $[\mathrm{M}+\mathrm{H}]^{+}$Calcd. for $\mathrm{C}_{21} \mathrm{H}_{21} \mathrm{NO}_{4} \mathrm{P}$ 382.1208; Found 382.1204.

Note: 1x aromatic signal not observed in ${ }^{13} \mathrm{C}-\mathrm{NMR}$ spectrum.

\section{Diphenyl benzoylphosphoramidate (3b)}<smiles>O=C(NP(=O)([18OH])Oc1ccccc1)c1ccccc1</smiles>

General procedure A was followed using diphenyl phosphite $(0.312 \mathrm{~g}, 1.0 \mathrm{mmol})$. The reaction mixture was purified using column chromatography gradient eluting from 100\% DCM to 5\% $\mathrm{MeOH} / \mathrm{DCM}$ to give $\mathbf{3 b}$ as a white solid (0.339 g, 96\%). ${ }^{1} \mathrm{H}$ NMR (300 $\left.\mathrm{MHz}, \mathrm{CDCl}_{3}\right) \delta 9.63$ (d, J 10.4 Hz, 1H), $8.01-7.93$ (m, 2H), $7.59-7.50$ (m, 1H), 7.43 - 7.34 (m, 2H), 7.29 - 7.20 $(\mathrm{m}, 8 \mathrm{H}), 7.20-7.09(\mathrm{~m}, 2 \mathrm{H}) ;{ }^{13} \mathrm{C}$ NMR $\left(75 \mathrm{MHz}, \mathrm{CDCl}_{3}\right) \delta 167.7$ (d, J 3.6 Hz), $150.2(\mathrm{~d}, J$ $6.7 \mathrm{~Hz}), 133.1,132.3$ (d, $J 11.1 \mathrm{~Hz}), 129.8$ (d, $J 1.1 \mathrm{~Hz}), 128.7,128.6,125.7$ (d, $J 1.5 \mathrm{~Hz})$, 120.7 (d, J 4.7 Hz); ${ }^{31} \mathrm{P}$ NMR (121 MHz, $\left.\mathrm{CDCl}_{3}\right) \delta-8.55 ; \mathrm{HRMS}(\mathrm{ESI}) \mathrm{m} / \mathrm{z}$ : $[\mathrm{M}+\mathrm{H}]^{+} \mathrm{Calcd}$. for $\mathrm{C}_{19} \mathrm{H}_{17} \mathrm{NO}_{4} \mathrm{P} 354.0895$; Found 354.0888. 
Matches previous reported spectral data. ${ }^{1}$

\section{Di-tert-butyl benzoylphosphoramidate (3c)}<smiles>CCCCOP(=O)(NC(=O)c1ccccc1)OCCC</smiles>

General procedure A was followed using di-tert-butyl phosphite $(0.194 \mathrm{~g}, 1.0 \mathrm{mmol})$. The reaction mixture was purified using column chromatography gradient eluting from $100 \%$ DCM to $5 \% \mathrm{MeOH} / \mathrm{DCM}$ to give $3 \mathrm{c}$ as a white solid $(0.292 \mathrm{~g}, 93 \%) .{ }^{1} \mathrm{H} \mathrm{NMR}\left(300 \mathrm{MHz}, \mathrm{CDCl}_{3}\right) \delta$ $9.31(\mathrm{~d}, J 10.0 \mathrm{~Hz}, 1 \mathrm{H}), 8.20-8.10(\mathrm{~m}, 2 \mathrm{H}), 7.57-7.48(\mathrm{~m}, 1 \mathrm{H}), 7.46-7.37(\mathrm{~m}, 2 \mathrm{H}), 1.53$ $(\mathrm{d}, J 0.6 \mathrm{~Hz}, 18 \mathrm{H}) ;{ }^{13} \mathrm{C} \mathrm{NMR}\left(75 \mathrm{MHz}, \mathrm{CDCl}_{3}\right) \delta 167.4$ (d, $\left.J 3.4 \mathrm{~Hz}\right), 133.8$ (d, J $\left.10.7 \mathrm{~Hz}\right)$, 132.3, 128.5, 128.4, 83.8 (d, J 7.6 Hz), 30.1 (d, J 4.4 Hz); ${ }^{31} \mathrm{P}$ NMR (121 MHz, $\left.\mathrm{CDCl}_{3}\right) \delta$ 10.94 (s); HRMS (ESI) m/z: [M+H] $]^{+}$Calcd. for $\mathrm{C}_{15} \mathrm{H}_{25} \mathrm{NO}_{4} \mathrm{P} 314.1521$; Found 314.1517.

\section{Dimethyl benzoylphosphoramidate (3d)}<smiles>COP(=O)(NC(=O)c1ccccc1)OC</smiles>

General procedure A was followed using dimethyl phosphite $(0.110 \mathrm{~g}, 1.0 \mathrm{mmol})$. The reaction mixture was purified using column chromatography gradient eluting from 100\% DCM to 5\% $\mathrm{MeOH} / \mathrm{DCM}$ to give 3d as a white solid (0.206 g, 90\%). ${ }^{1} \mathrm{H}$ NMR (300 MHz, $\left.\mathrm{CDCl}_{3}\right) \delta 9.44$ $(\mathrm{d}, J 9.0 \mathrm{~Hz}, 1 \mathrm{H}), 8.17-8.04(\mathrm{~m}, 2 \mathrm{H}), 7.62-7.50(\mathrm{~m}, 1 \mathrm{H}), 7.50-7.41(\mathrm{~m}, 2 \mathrm{H}), 3.88(\mathrm{~d}, J 11.7$ $\mathrm{Hz}, 6 \mathrm{H}) .{ }^{13} \mathrm{C} \mathrm{NMR}\left(75 \mathrm{MHz}, \mathrm{CDCl}_{3}\right) \delta 168.2(\mathrm{~d}, J 2.8 \mathrm{~Hz}), 133.0,132.4$ (d, J 10.6 Hz), 128.7, 128.5, 54.6 (d, J 5.9 Hz). ${ }^{31} \mathrm{P}$ NMR (121 MHz, $\left.\mathrm{CDCl}_{3}\right) \delta 2.25$. HRMS (ESI) m/z: $[\mathrm{M}+\mathrm{H}]^{+}$ Calcd. for $\mathrm{C}_{9} \mathrm{H}_{13} \mathrm{NO}_{4} \mathrm{P} 230.0582$; Found 230.0579. 


\section{$N$-(Diphenylphosphoryl)benzamide (3e)}<smiles>O=C(NP(=O)(c1ccccc1)c1ccccc1)c1ccccc1</smiles>

General procedure A was followed using diphenylphosphine oxide $(0.202 \mathrm{~g}, 1.0 \mathrm{mmol})$. The reaction mixture was purified using column chromatography gradient eluting from $100 \%$ DCM to $5 \% \mathrm{MeOH} / \mathrm{DCM}$ to give $3 \mathrm{e}$ as a white solid (339 mg, 94\%). ${ }^{1} \mathrm{H} \mathrm{NMR}\left(300 \mathrm{MHz}, \mathrm{CDCl}_{3}\right) \delta$ $9.68(\mathrm{~d}, J 4.0 \mathrm{~Hz}, 1 \mathrm{H}), 8.13-8.02(\mathrm{~m}, 2 \mathrm{H}), 7.98-7.85(\mathrm{~m}, 4 \mathrm{H}), 7.60-7.31(\mathrm{~m}, 9 \mathrm{H}) ;{ }^{13} \mathrm{C} \mathrm{NMR}$ $\left(75 \mathrm{MHz}, \mathrm{CDCl}_{3}\right) \delta 169.0$ (d, $\left.J 1.8 \mathrm{~Hz}\right), 132.8,132.6$ (d, $\left.J 2.9 \mathrm{~Hz}\right), 132.2,132.0,130.0,128.6$, 128.4; ${ }^{31} \mathrm{P} \mathrm{NMR}\left(121 \mathrm{MHz}, \mathrm{CDCl}_{3}\right) \delta 28.94 ; \mathrm{HRMS}(\mathrm{ESI}) \mathrm{m} / \mathrm{z}$ : $[\mathrm{M}+\mathrm{H}]^{+}$Calcd. for $\mathrm{C}_{19} \mathrm{H}_{17} \mathrm{NO}_{2} \mathrm{P}$ 322.0997; Found 322.0991.

Matches previously reported spectral data. ${ }^{2}$

\section{Ethyl $N$-benzoyl- $P$-phenylphosphonamidate (3f)}<smiles>CCOP(=O)(NC(=O)c1ccccc1)c1ccccc1</smiles>

General procedure A was followed using ethyl phenylphosphinate $(0.170 \mathrm{~g}, 1.0 \mathrm{mmol})$. The reaction mixture was purified using column chromatography gradient eluting from 100\% DCM to $5 \% \mathrm{MeOH} / \mathrm{DCM}$ to give $3 \mathrm{f}$ as a white solid $(0.267 \mathrm{~g}, 92 \%) .{ }^{1} \mathrm{H} \mathrm{NMR}\left(300 \mathrm{MHz}, \mathrm{CDCl}_{3}\right) \delta$ $9.66(\mathrm{~d}, J 6.1 \mathrm{~Hz}, 1 \mathrm{H}), 8.17$ - 7.99 (m, 4H), 7.60 - 7.35 (m, 6H), 4.45 - 4.15 (m, 2H), 1.49 $1.37(\mathrm{~m}, 3 \mathrm{H}) ;{ }^{13} \mathrm{C}$ NMR $\left(75 \mathrm{MHz}, \mathrm{CDCl}_{3}\right) \delta 168.1(\mathrm{~d}, J 1.8 \mathrm{~Hz}), 132.9$ - $132.6(\mathrm{~m}), 132.3$, 132.1, 130.5, 128.6, 128.6 - 128.4 (m), 128.3, 128.1, 62.1 (d, J $6.8 \mathrm{~Hz}), 16.4$ (d, J 6.8 Hz); ${ }^{31} \mathrm{P}$ NMR (121 MHz, $\left.\mathrm{CDCl}_{3}\right) \delta$ 17.58; HRMS (ESI) m/z: $[\mathrm{M}+\mathrm{H}]^{+}$Calcd. for $\mathrm{C}_{15} \mathrm{H}_{17} \mathrm{NO}_{3} \mathrm{P}$ 290.0946; Found 290.0941. 


\section{Dibenzyl diphenyl imidodiphosphate (5a)}<smiles>O=P(NP(=O)(Oc1ccccc1)Oc1ccccc1)(OCc1ccccc1)Oc1ccccc1</smiles>

General procedure B was followed using dibenzyl phosphite $(0.262 \mathrm{~g}, 1.0 \mathrm{mmol})$. The reaction mixture was purified using column chromatography gradient eluting from 100\% DCM to 5\% $\mathrm{MeOH} / \mathrm{DCM}$ to give 5a as a pale yellow oil $(0.447 \mathrm{~g}, 88 \%) .{ }^{1} \mathrm{H} \mathrm{NMR}\left(300 \mathrm{MHz}, \mathrm{CDCl}_{3}\right) \delta$ $7.32-7.07$ (m, 20H), $5.03-4.84(\mathrm{~m}, 4 \mathrm{H}) ;{ }^{13} \mathrm{C} \mathrm{NMR}\left(75 \mathrm{MHz}, \mathrm{CDCl}_{3}\right) \delta 150.5$ (d, J $\left.6.9 \mathrm{~Hz}\right)$, 135.7 (d, J 8.2 Hz), 129.7 (d, J 1.1 Hz), 128.5, 128.4, 128.0, 125.4 (d, J 1.4 Hz), 120.8 (d, J 4.8 $\mathrm{Hz}), 69.1$ (d, $J 5.4 \mathrm{~Hz}) ;{ }^{13} \mathrm{C}$ NMR $\left(75 \mathrm{MHz}, \mathrm{CDCl}_{3}\right) \delta 150.45$ (d, $\left.J 6.9 \mathrm{~Hz}\right), 135.67$ (d, $J 8.2$ Hz), 129.73 (d, J 1.1 Hz), 128.53, 128.42, 127.98, 125.44 (d, J 1.4 Hz), 120.80 (d, J 4.8 Hz), 69.08 (d, J 5.4 Hz); ${ }^{31} \mathrm{P}$ NMR (121 MHz, CDCl 3$) \delta-0.58$ (d, J $\left.6.1 \mathrm{~Hz}\right),-8.50$ (d, J $\left.6.4 \mathrm{~Hz}\right)$; HRMS (ESI) m/z: [M+H] $]^{+}$Calcd. for $\mathrm{C}_{26} \mathrm{H}_{26} \mathrm{NO}_{6} \mathrm{P}_{2}$ 510.1235; Found 510.1236.

\section{Tetraphenyl imidodiphosphate (5b)}<smiles>O=P(NP(=O)(Oc1ccccc1)Oc1ccccc1)(Oc1ccccc1)Oc1ccccc1</smiles>

General procedure B was followed using diphenyl phosphite $(75 \%)(0.312 \mathrm{~g}, 1.0 \mathrm{mmol})$. The reaction mixture was purified using column chromatography gradient eluting from 100\% DCM to $5 \% \mathrm{MeOH} / \mathrm{DCM}$ to give $\mathbf{5 b}$ as a white solid (0.406 g, 84\%). ${ }^{1} \mathrm{H} \mathrm{NMR}\left(300 \mathrm{MHz}, \mathrm{CDCl}_{3}\right) \delta$ $7.25-7.08(\mathrm{~m}, 20 \mathrm{H}) ;{ }^{13} \mathrm{C}$ NMR $\left(75 \mathrm{MHz}, \mathrm{CDCl}_{3}\right) \delta 150.50-150.15(\mathrm{~m}), 129.72,125.43$, $120.76-120.44(\mathrm{~m}) ;{ }^{31} \mathrm{P}$ NMR $\left(121 \mathrm{MHz}, \mathrm{CDCl}_{3}\right) \delta-9.83$.

Matches previously reported spectral data. ${ }^{2}$ 


\section{Di-tert-butyl diphenyl imidodiphosphate (5c)}<smiles>CC(C)(C)OP(=O)(NP(=O)(Oc1ccccc1)Oc1ccccc1)OC(C)(C)C</smiles>

General procedure B was followed using di-tert-butyl phosphite $(0.194 \mathrm{~g}, 1.0 \mathrm{mmol})$. The reaction mixture was purified using column chromatography gradient eluting from $100 \% \mathrm{DCM}$ to $5 \% \mathrm{MeOH} / \mathrm{DCM}$ to give $\mathbf{5 c}$ as a pale yellow oil $(0.441 \mathrm{~g}, 89 \%) .{ }^{1} \mathrm{H} \mathrm{NMR}\left(300 \mathrm{MHz}, \mathrm{CDCl}_{3}\right)$ $\delta 7.38-7.23(\mathrm{~m}, 8 \mathrm{H}), 7.19-7.07(\mathrm{~m}, 2 \mathrm{H}), 1.42(\mathrm{~s}, 18 \mathrm{H}) ;{ }^{13} \mathrm{C} \mathrm{NMR}\left(75 \mathrm{MHz}, \mathrm{CDCl}_{3}\right) \delta 150.8$ (d, J 7.3 Hz), 129.5, 124.9, 120.7 (d, J 4.8 Hz), 29.9 (d, J4.1 Hz); ${ }^{31} \mathrm{P}$ NMR (121 MHz, CDCl $)$ $\delta$-8.7, -9.4; HRMS (ESI) m/z: [M+H] ${ }^{+}$Calcd. For $\mathrm{C}_{20} \mathrm{H}_{30} \mathrm{NO}_{6} \mathrm{P}_{2} 442.1548$; Found 442.1542.

Note: Product was found to be unstable after prolonged storage at room temperature.

\section{Dimethyl diphenyl imidodiphosphate (5d)}<smiles>COP(=O)(NP(=O)(OC)Oc1ccccc1)Oc1ccccc1</smiles>

General procedure B was followed using dimethyl phosphite $(0.110 \mathrm{~g}, 1.0 \mathrm{mmol})$. The reaction mixture was purified using column chromatography gradient eluting from 100\% DCM to 5\% $\mathrm{MeOH} / \mathrm{DCM}$ to give $\mathbf{5 d}$ as a white solid (0.303 g, 85\%). ${ }^{1} \mathrm{H}$ NMR (300 $\left.\mathrm{MHz}, \mathrm{CDCl}_{3}\right) \delta 7.34-$ $7.27(\mathrm{~m}, 4 \mathrm{H}), 7.22-7.14(\mathrm{~m}, 1 \mathrm{H}), 3.63(\mathrm{~d}, J 11.7 \mathrm{~Hz}, 3 \mathrm{H}) ;{ }^{13} \mathrm{C} \mathrm{NMR}\left(75 \mathrm{MHz}, \mathrm{CDCl}_{3}\right) \delta$ 150.50 (d, J 7.0 Hz), 129.79 (d, J 1.0 Hz), 125.53 (d, J 1.5 Hz), 120.74 (d, J 4.9 Hz), 54.10 (d, $J 5.7 \mathrm{~Hz}) ;{ }^{31} \mathrm{P}$ NMR $\left(121 \mathrm{MHz}, \mathrm{CDCl}_{3}\right) \delta 2.03$ (d, $\left.J 6.4 \mathrm{~Hz}\right),-8.72$ (d, $\left.J 6.4 \mathrm{~Hz}\right)$; HRMS (ESI) $\mathrm{m} / \mathrm{z}:[\mathrm{M}+\mathrm{H}]^{+}$Calcd. For $\mathrm{C}_{14} \mathrm{H}_{18} \mathrm{NO}_{6} \mathrm{P}_{2}$ 358.0609; Found 358.0602 . 


\section{Diphenyl diphenylphosphorylphosphoramidate (5e)}<smiles>O=P(NP(=O)(c1ccccc1)c1ccccc1)(Oc1ccccc1)c1ccccc1</smiles>

General procedure B was followed using diphenylphosphine oxide (0.202 g, $1.0 \mathrm{mmol})$. The reaction mixture was purified using column chromatography gradient eluting from $100 \%$ DCM to $5 \% \mathrm{MeOH} / \mathrm{DCM}$ to give $5 \mathrm{e}$ as a beige solid $(0.414 \mathrm{~g}, 92 \%) .{ }^{1} \mathrm{H} \mathrm{NMR}\left(300 \mathrm{MHz}, \mathrm{CDCl}_{3}\right) \delta$ $8.7(\mathrm{~s}, 1 \mathrm{H}), 7.8-7.6(\mathrm{~m}, 4 \mathrm{H}), 7.5-7.4(\mathrm{~m}, 2 \mathrm{H}), 7.4-7.2(\mathrm{~m}, 4 \mathrm{H}), 7.2-7.0(\mathrm{~m}, 10 \mathrm{H}) ;{ }^{13} \mathrm{C}$ NMR (75 MHz, $\left.\mathrm{CDCl}_{3}\right) \delta 150.7$ (d, J 7.1 Hz), 132.1 (d, J 2.9 Hz), 132.0 (dd, J 131.2, $2.8 \mathrm{~Hz}$ ), 131.9 (d, $J 11.1 \mathrm{~Hz}), 129.5,128.3$ (d, J $13.7 \mathrm{~Hz}), 125.0$ (d, J 1.5 Hz), 120.8 (d, J 4.9 Hz); ${ }^{31} \mathrm{P}$ NMR (121 MHz, $\left.\mathrm{CDCl}_{3}\right) \delta 24.4,-6.2$; HRMS (ESI) m/z: $[\mathrm{M}+\mathrm{H}]^{+}$Calcd. for $\mathrm{C}_{24} \mathrm{H}_{22} \mathrm{NO}_{4} \mathrm{P}_{2}$ 450.1024; Found 450.1025.

\section{Diphenyl ethoxy(phenyl)phosphorylphosphoramidate (5f)}<smiles>CCOP(=O)(NP(=O)(Oc1ccccc1)Oc1ccccc1)c1ccccc1</smiles>

General procedure B was followed using ethyl phenylphosphinate $(0.170 \mathrm{~g}, 1.0 \mathrm{mmol})$. The reaction mixture was purified using column chromatography gradient eluting from $100 \%$ DCM to $5 \% \mathrm{MeOH} / \mathrm{DCM}$ to give $\mathbf{5 f}$ as a white solid $(0.383 \mathrm{~g}, 92 \%) .{ }^{1} \mathrm{H} \mathrm{NMR}\left(300 \mathrm{MHz}, \mathrm{CDCl}_{3}\right) \delta$ $7.91-7.69(\mathrm{~m}, 2 \mathrm{H}), 7.56-6.92(\mathrm{~m}, 13 \mathrm{H}), 4.23-3.81(\mathrm{~m}, 2 \mathrm{H}), 1.24(\mathrm{t}, J 7.1 \mathrm{~Hz}, 3 \mathrm{H}) ;{ }^{13} \mathrm{C}$ NMR (75 MHz, $\left.\mathrm{CDCl}_{3}\right) \delta 150.5(\mathrm{dd}, J 20.1,6.9 \mathrm{~Hz}), 132.3(\mathrm{~d}, J 3.1 \mathrm{~Hz}), 131.6(\mathrm{~d}, J 10.6 \mathrm{~Hz})$, 129.6 (d, $J 11.2 \mathrm{~Hz}), 128.2$ (d, J 15.4 Hz), 125.6 - 124.9 (m), 120.9 (d, J 4.7 Hz), 120.5 (d, J $4.9 \mathrm{~Hz}), 61.9$ (d, J 6.4 Hz), 16.2 (d, J 7.0 Hz); ${ }^{31} \mathrm{P}$ NMR (121 MHz, $\left.\mathrm{CDCl}_{3}\right) \delta$ 16.24, -8.44; HRMS (ESI) m/z: [M+H] $]^{+}$Calcd. for $\mathrm{C}_{20} \mathrm{H}_{22} \mathrm{NO}_{5} \mathrm{P}_{2}$ 418.0973; Found 418.0969. 


\section{Dibenzyl thiophene-2-carbonylphosphoramidate (3g)}<smiles>O=C(NP(=O)(OCc1ccccc1)OCc1ccccc1)c1ccccc1</smiles>

General procedure A was followed using thiophene-2-carbonyl azide (0.153 g, $1.0 \mathrm{mmol})$. The reaction mixture was purified using column chromatography gradient eluting from 100\% DCM to $5 \% \mathrm{MeOH} / \mathrm{DCM}$ to give $\mathbf{3 g}$ as a white solid $(0.375 \mathrm{~g}, 96 \%) .{ }^{1} \mathrm{H} \mathrm{NMR}\left(300 \mathrm{MHz}, \mathrm{CDCl}_{3}\right) \delta$ $9.68(\mathrm{~d}, J 9.1 \mathrm{~Hz}, 1 \mathrm{H}), 8.01(\mathrm{dd}, J 3.9,1.1 \mathrm{~Hz}, 1 \mathrm{H}), 7.62-7.55(\mathrm{~m}, 1 \mathrm{H}), 7.43-7.34(\mathrm{~m}, 4 \mathrm{H})$, $7.34-7.22(\mathrm{~m}, 6 \mathrm{H}), 7.05(\mathrm{dd}, J 5.0,3.8 \mathrm{~Hz}, 1 \mathrm{H}), 5.33-5.16(\mathrm{~m}, 4 \mathrm{H}) ;{ }^{13} \mathrm{C} \mathrm{NMR}(75 \mathrm{MHz}$, $\left.\mathrm{CDCl}_{3}\right) \delta 162.6(\mathrm{~d}, J 1.9 \mathrm{~Hz}), 138.1$ (d, $\left.J 13.1 \mathrm{~Hz}\right), 135.6$ (d, $\left.J 7.0 \mathrm{~Hz}\right), 132.9,131.6,128.5$, 128.5, 128.1, 128.0, 69.9 (d, J 5.9 Hz); $\left.{ }^{31} \mathrm{P} \mathrm{NMR} \mathrm{(121} \mathrm{MHz,} \mathrm{CDCl}_{3}\right) \delta-1.41 ; \mathrm{HRMS}(\mathrm{ESI}) \mathrm{m} / \mathrm{z}$ : $[\mathrm{M}+\mathrm{H}]^{+}$Calcd. for $\mathrm{C}_{19} \mathrm{H}_{19} \mathrm{NO}_{4} \mathrm{PS}$ 388.0772; Found 388.0769.

\section{Dibenzyl furan-2-carbonylphosphoramidate (3h)}<smiles>O=C(NP(=O)(OCc1ccccc1)OCc1ccccc1)c1ccccc1</smiles>

General procedure A was followed using furan-2-carbonyl azide $(0.137 \mathrm{~g}, 1.0 \mathrm{mmol})$. The reaction mixture was purified using column chromatography gradient eluting from $100 \% \mathrm{DCM}$ to $5 \% \mathrm{MeOH} / \mathrm{DCM}$ to give $\mathbf{3 h}$ as a white solid $(0.335 \mathrm{~g}, 90 \%) .{ }^{1} \mathrm{H} \mathrm{NMR}\left(300 \mathrm{MHz}, \mathrm{CDCl}_{3}\right) \delta$ 8.42 (d, J 9.6 Hz, 1H), 7.49 (dd, $J 1.7,0.8 \mathrm{~Hz}, 1 \mathrm{H}), 7.44-7.23(\mathrm{~m}, 11 \mathrm{H}), 6.50$ (dd, $J 3.6,1.7$ $\mathrm{Hz}, 1 \mathrm{H}), 5.32-5.15(\mathrm{~m}, 4 \mathrm{H}) ;{ }^{13} \mathrm{C} \mathrm{NMR}\left(75 \mathrm{MHz}, \mathrm{CDCl}_{3}\right) \delta 158.0(\mathrm{~d}, J 1.1 \mathrm{~Hz}), 146.4(\mathrm{~d}, J$ 12.7 Hz), 145.8, 135.6 (d, J 7.2 Hz), 128.6, 128.6, 128.1, 117.3, 112.7, 69.8 (d, J 5.7 Hz); ${ }^{31} \mathrm{P}$ $\mathrm{NMR}\left(121 \mathrm{MHz}, \mathrm{CDCl}_{3}\right) \delta$-1.79; HRMS (ESI) m/z: $[\mathrm{M}+\mathrm{H}]^{+}$Calcd. for $\mathrm{C}_{19} \mathrm{H}_{19} \mathrm{NO}_{5} \mathrm{P} 372.1001$; Found 372.0997. 
<smiles>O=C(/C=C/c1ccccc1)NP(=O)(Cc1ccccc1)OCc1ccccc1</smiles>

General procedure A was followed using cinnamoyl azide $(0.173 \mathrm{~g}, 1.0 \mathrm{mmol})$. The reaction mixture was purified using column chromatography gradient eluting from 100\% DCM to 5\% $\mathrm{MeOH} / \mathrm{DCM}$ to give 3i as a white solid (0.391 g, 96\%). ${ }^{1} \mathrm{H}$ NMR (300 $\left.\mathrm{MHz}, \mathrm{CDCl}_{3}\right) \delta 9.25$ (d, J 10.3 Hz, 1H), $7.73(\mathrm{~d}, J 15.8 \mathrm{~Hz}, 1 \mathrm{H}), 7.55-7.47(\mathrm{~m}, 2 \mathrm{H}), 7.44-7.34(\mathrm{~m}, 7 \mathrm{H}), 7.34-$ $7.23(\mathrm{~m}, 6 \mathrm{H}), 6.62(\mathrm{~d}, J 15.8 \mathrm{~Hz}, 1 \mathrm{H}), 5.32-5.14(\mathrm{~m}, 4 \mathrm{H}) ;{ }^{13} \mathrm{C} \mathrm{NMR}\left(75 \mathrm{MHz}, \mathrm{CDCl}_{3}\right) \delta 167.1$ (d, J 2.2 Hz), 144.4 (d, J2.1 Hz), 135.7 (d, J 7.1 Hz), 134.5, 130.5, 129.0, 128.7, 128.6, 128.4, 128.1, 119.8 (d, $J 11.9 \mathrm{~Hz}), 69.8$ (d, $J 5.7 \mathrm{~Hz}) ;{ }^{31} \mathrm{P}$ NMR (121 MHz, $\left.\mathrm{CDCl}_{3}\right) \delta-0.61$; HRMS (ESI) m/z: $[\mathrm{M}+\mathrm{H}]^{+}$Calcd. for $\mathrm{C}_{23} \mathrm{H}_{23} \mathrm{NO}_{4} \mathrm{P}$ 408.1365; Found 408.1362.

\section{Dibenzyl picolinoylphosphoramidate (3j)}<smiles>O=C(NP(=O)(OCc1ccccc1)OCc1ccccc1)c1ccccn1</smiles>

General procedure A was followed using picolinoyl azide $(0.148 \mathrm{~g}, 1.0 \mathrm{mmol})$. The reaction mixture was purified using column chromatography gradient eluting from 100\% DCM to 5\% $\mathrm{MeOH} / \mathrm{DCM}$ to give $\mathbf{3 j}$ as a beige solid $(0.360 \mathrm{~g}, 94 \%) .{ }^{1} \mathrm{H} \mathrm{NMR}\left(300 \mathrm{MHz}, \mathrm{CDCl}_{3}\right) \delta 9.83$ $(\mathrm{d}, J 8.8 \mathrm{~Hz}, 1 \mathrm{H}), 9.21(\mathrm{dd}, J 2.4,0.9 \mathrm{~Hz}, 1 \mathrm{H}), 8.76$ (dd, $J 4.9,1.6 \mathrm{~Hz}, 1 \mathrm{H}), 8.30$ (ddd, $J$ 8.0, $2.3,1.6 \mathrm{~Hz}, 1 \mathrm{H}), 7.41-7.33(\mathrm{~m}, 4 \mathrm{H}), 7.33-7.23(\mathrm{~m}, 6 \mathrm{H}), 5.33-5.14(\mathrm{~m}, 4 \mathrm{H}) ;{ }^{13} \mathrm{C} \mathrm{NMR}(75$ $\left.\mathrm{MHz}, \mathrm{CDCl}_{3}\right) \delta 166.52(\mathrm{~d}, J 2.7 \mathrm{~Hz}), 152.85,149.69,136.48,135.50$ (d, $\left.J 6.6 \mathrm{~Hz}\right), 128.72$, 128.70, 128.52 (d, $J 11.0 \mathrm{~Hz}), 128.18,123.43,70.11$ (d, $J 5.9 \mathrm{~Hz}) ;{ }^{31} \mathrm{P}$ NMR $\left(121 \mathrm{MHz}, \mathrm{CDCl}_{3}\right)$ $\delta$-1.29; HRMS (ESI) m/z: [M+H] $]^{+}$Calcd. for $\mathrm{C}_{20} \mathrm{H}_{20} \mathrm{~N}_{2} \mathrm{O}_{4} \mathrm{P}$ 383.1161; Found: 383.1164 . 


\section{Dibenzyl $1 H$-indole-2-carbonylphosphoramidate (3k)}<smiles>O=C(NP(=O)(OCc1ccccc1)OCc1ccccc1)c1cc2ccccc2[nH]1</smiles>

General procedure A was followed using $1 H$-indole-2-carbonyl azide $(0.186 \mathrm{~g}, 1.0 \mathrm{mmol})$. The reaction mixture was purified using column chromatography gradient eluting from $100 \%$ DCM to $5 \% \mathrm{MeOH} / \mathrm{DCM}$ to give $3 \mathrm{k}$ as a beige solid (0.405 g, 96\%). ${ }^{1} \mathrm{H} \mathrm{NMR}\left(300 \mathrm{MHz}, \mathrm{CDCl}_{3}\right) \delta$ 10.05 (s, 1H), 9.69 (d, J9.0 Hz, 1H), $7.73-7.66(\mathrm{~m}, 1 \mathrm{H}), 7.54-7.49$ (m, 1H), $7.44-7.21$ (m, $12 \mathrm{H}), 7.16(\mathrm{ddd}, J 8.0,6.6,1.4 \mathrm{~Hz}, 1 \mathrm{H}), 5.38-5.22(\mathrm{~m}, 4 \mathrm{H}) ;{ }^{13} \mathrm{C} \mathrm{NMR}\left(75 \mathrm{MHz}, \mathrm{CDCl}_{3}\right) \delta$ 161.9 (d, J 2.0 Hz), 137.5, 135.4 (d, J 6.7 Hz), 129.4 (d, J 13.2 Hz), 128.7, 128.7, 128.2, 127.5, 125.6, 122.9, 120.9, 112.3, 109.0, 70.3 (d, $J 5.8 \mathrm{~Hz}$ ); ${ }^{31} \mathrm{P}$ NMR (121 MHz, $\left.\mathrm{CDCl}_{3}\right) \delta-0.93$; HRMS (ESI) m/z: [M+H] $]^{+}$Calcd. for $\mathrm{C}_{23} \mathrm{H}_{22} \mathrm{~N}_{2} \mathrm{O}_{4} \mathrm{P} 421.1317$; Found 421.1313.

\section{Dibenzyl $1 H$-indole-3-carbonylphosphoramidate (31)}<smiles>O=C(NP(=O)(OCc1ccccc1)OCc1ccccc1)c1c[nH]c2ccccc12</smiles>

General procedure A was followed using $1 H$-indole-3-carbonyl azide $(0.186 \mathrm{~g}, 1.0 \mathrm{mmol})$. The reaction mixture was purified using column chromatography gradient eluting from $100 \% \mathrm{DCM}$ to $5 \% \mathrm{MeOH} / \mathrm{DCM}$ to give $3 \mathrm{l}$ as a beige solid (0.403 g, 96\%). ${ }^{1} \mathrm{H}$ NMR (300 MHz, DMSO- $d 6$ ) $\delta 9.63(\mathrm{~s}, 1 \mathrm{H}), 8.43(\mathrm{~d}, J 2.8 \mathrm{~Hz}, 1 \mathrm{H}), 8.22-8.12(\mathrm{~m}, 1 \mathrm{H}), 7.54-7.27(\mathrm{~m}, 11 \mathrm{H}), 7.24-7.12$ $(\mathrm{m}, 2 \mathrm{H}), 5.24-5.09(\mathrm{~m}, 4 \mathrm{H}) ;{ }^{13} \mathrm{C}$ NMR $\left(75 \mathrm{MHz}, \mathrm{DMSO}-d_{6}\right) \delta 165.0(\mathrm{~d}, J 1.6 \mathrm{~Hz}), 136.5(\mathrm{~d}$, $J 7.5 \mathrm{~Hz}), 136.3,131.2,128.4,128.1,127.7,126.4$ (d, $J 2.1 \mathrm{~Hz}), 122.5,121.2,121.0,112.1$, 108.9 (d, $J 11.9 \mathrm{~Hz}), 67.9$ (d, $J 5.3 \mathrm{~Hz}) ;{ }^{31} \mathrm{P}$ NMR (121 MHz, DMSO-d6) $\delta 1.5$; HRMS (ESI) $\mathrm{m} / \mathrm{z}:[\mathrm{M}+\mathrm{H}]^{+}$Calcd. for $\mathrm{C}_{23} \mathrm{H}_{22} \mathrm{~N}_{2} \mathrm{O}_{4} \mathrm{P}$ 421.1317; Found 421.1312. 


\section{Dibenzyl (4-nitrobenzoyl)phosphoramidate (3m)}<smiles>O=C(NP(=O)(Cc1ccccc1)OCc1ccccc1)c1ccc([N+](=O)[O-])cc1</smiles>

General procedure A was followed using 4-nitrobenzoyl azide (0.192 g, $1.0 \mathrm{mmol})$. The reaction mixture was purified using column chromatography gradient eluting from $100 \% \mathrm{DCM}$ to $5 \% \mathrm{MeOH} / \mathrm{DCM}$ to give $\mathbf{3 m}$ as a white solid (0.396 g, 93\%). ${ }^{1} \mathrm{H}$ NMR (300 $\left.\mathrm{MHz}, \mathrm{CDCl}_{3}\right)$ $\delta 9.75(\mathrm{~d}, J 8.8 \mathrm{~Hz}, 1 \mathrm{H}), 8.25-8.16(\mathrm{~m}, 2 \mathrm{H}), 8.16-8.07$ (m, 2H), $7.42-7.26(\mathrm{~m}, 10 \mathrm{H}), 5.33$ - $5.16(\mathrm{~m}, 4 \mathrm{H}) ;{ }^{13} \mathrm{C} \mathrm{NMR}\left(75 \mathrm{MHz}, \mathrm{CDCl}_{3}\right) \delta 166.3$ (d, J2.4 Hz), 150.4, 137.8 (d, J $\left.11.0 \mathrm{~Hz}\right)$, 135.4 (d, $J 6.2 \mathrm{~Hz}), 129.8,128.9,128.8,128.2,123.7,70.3$ (d, $J 6.0 \mathrm{~Hz}) ;{ }^{31} \mathrm{P}$ NMR $(121 \mathrm{MHz}$, $\left.\mathrm{CDCl}_{3}\right) \delta$-1.68; HRMS (ESI) m/z: $[\mathrm{M}+\mathrm{H}]^{+}$Calcd. for $\mathrm{C}_{21} \mathrm{H}_{20} \mathrm{~N}_{2} \mathrm{O}_{6} \mathrm{P}$ 427.1059; Found: 427.1054 .

\section{Dibenzyl (4-(dimethylamino)benzoyl)phosphoramidate (3n)}<smiles>CN(C)c1ccc(C(=O)NP(=O)(OCc2ccccc2)Oc2ccccc2)cc1</smiles>

General procedure A was followed using 4-(dimethylamino)benzoyl azide (0.190 g, $1.0 \mathrm{mmol})$. The reaction mixture was purified using column chromatography gradient eluting from $100 \%$ DCM to $5 \% \mathrm{MeOH} / \mathrm{DCM}$ to give $3 \mathrm{n}$ as a beige solid (0.395 g, 93\%). ${ }^{1} \mathrm{H}$ NMR (300 MHz, $\left.\mathrm{CDCl}_{3}\right) \delta 8.50(\mathrm{~d}, J 9.6 \mathrm{~Hz}, 1 \mathrm{H}), 7.91-7.81(\mathrm{~m}, 2 \mathrm{H}), 7.43-7.34(\mathrm{~m}, 4 \mathrm{H}), 7.34-7.24(\mathrm{~m}, 6 \mathrm{H})$, $6.73-6.63(\mathrm{~m}, 2 \mathrm{H}), 5.31-5.15(\mathrm{~m}, 4 \mathrm{H}), 3.03(\mathrm{~s}, 6 \mathrm{H}) ;{ }^{13} \mathrm{C} \mathrm{NMR}\left(75 \mathrm{MHz}, \mathrm{CDCl}_{3}\right) \delta 167.5(\mathrm{~d}$, $J 2.4 \mathrm{~Hz}), 153.0,136.0$ (d, J 7.3 Hz), 130.2, 128.6, 128.4, 128.1, 111.5, 69.6 (d, J 5.7 Hz), 40.5; ${ }^{31} \mathrm{P}$ NMR (121 MHz, $\left.\mathrm{CDCl}_{3}\right) \delta$ 0.09; HRMS (ESI) m/z: [M+H] ${ }^{+}$Calcd. for $\mathrm{C}_{23} \mathrm{H}_{26} \mathrm{~N}_{2} \mathrm{O}_{4} \mathrm{P}$ 425.1630; Found 425.1626.

Note: 1x aromatic signals not observed in ${ }^{13} \mathrm{C}-\mathrm{NMR}$ spectrum. 


\section{Dibenzyl (3-acetylbenzoyl)phosphoramidate (3p)}<smiles>CC(=O)c1cccc(C(=O)NP(=O)(Cc2ccccc2)OCc2ccccc2)c1</smiles>

General procedure A was followed using 3-acetylbenzoyl azide (0.189 g, $1.0 \mathrm{mmol})$. The reaction mixture was purified using column chromatography gradient eluting from $100 \%$ DCM to $5 \% \mathrm{MeOH} / \mathrm{DCM}$ to give $3 \mathrm{p}$ as a white solid (0.395 g, 93\%). $\left.{ }^{1} \mathrm{H} \mathrm{NMR} \mathrm{(300} \mathrm{MHz}, \mathrm{CDCl}_{3}\right) \delta$ $9.81(\mathrm{~d}, J 8.9 \mathrm{~Hz}, 1 \mathrm{H}), 8.65-8.58(\mathrm{~m}, 1 \mathrm{H}), 8.27$ - $8.19(\mathrm{~m}, 1 \mathrm{H}), 8.19-8.12(\mathrm{~m}, 1 \mathrm{H}), 7.59-$ $7.49(\mathrm{~m}, 1 \mathrm{H}), 7.42-7.32(\mathrm{~m}, 4 \mathrm{H}), 7.32-7.21(\mathrm{~m}, 6 \mathrm{H}), 5.33-5.18(\mathrm{~m}, 4 \mathrm{H}), 2.57(\mathrm{~s}, 3 \mathrm{H}) ;{ }^{13} \mathrm{C}$ NMR (75 MHz, $\left.\mathrm{CDCl}_{3}\right) \delta 197.6,167.4(\mathrm{~d}, J 2.6 \mathrm{~Hz}), 137.5,135.6$ (d, J 6.7 Hz), 133.2, 133.0 (d, $J 10.7 \mathrm{~Hz}), 132.2,129.1,128.7,128.7,128.6,128.1,70.1$ (d, $J 6.0 \mathrm{~Hz}), 26.9 ;{ }^{31} \mathrm{P}$ NMR (121 $\mathrm{MHz}, \mathrm{CDCl}_{3}$ ) $\delta$-1.22; HRMS (ESI) m/z: $[\mathrm{M}+\mathrm{H}]^{+}$Calcd. for $\mathrm{C}_{23} \mathrm{H}_{23} \mathrm{NO}_{5} \mathrm{P}$ 424.1314; Found 424.1312.

\section{Dibenzyl (4-benzoylbenzoyl)phosphoramidate (3q)}<smiles>O=C(NP(=O)(OCc1ccccc1)OCc1ccccc1)c1ccccc1</smiles>

General procedure A was followed using 4-benzoylbenzoyl azide $(0.251 \mathrm{~g}, 1.0 \mathrm{mmol})$. The reaction mixture was purified using column chromatography gradient eluting from 100\% DCM to $5 \% \mathrm{MeOH} / \mathrm{DCM}$ to yield $\mathbf{3 q}$ as a beige solid (0.475 g, 96\%). ${ }^{1} \mathrm{H} \mathrm{NMR}\left(300 \mathrm{MHz}, \mathrm{CDCl}_{3}\right) \delta$ $9.41(\mathrm{~d}, J 9.2 \mathrm{~Hz}, 1 \mathrm{H}), 8.07$ (d, J 8.1 Hz, 2H), $7.84-7.76$ (m, 4H), $7.68-7.59$ (m, 1H), 7.57 $7.47(\mathrm{~m}, 2 \mathrm{H}), 7.44-7.19(\mathrm{~m}, 10 \mathrm{H}), 5.34-5.16(\mathrm{~m}, 4 \mathrm{H}) ;{ }^{13} \mathrm{C} \mathrm{NMR}\left(75 \mathrm{MHz}, \mathrm{CDCl}_{3}\right) \delta 196.5$, 167.9 (d, J 2.7 Hz), 141.6, 137.6, 136.0 (d, J 7.9 Hz), 133.5, 130.6, 130.4, 129.1, 129.0, 128.9, 128.5, 70.5 (d, $J 5.9 \mathrm{~Hz}) ;{ }^{31} \mathrm{P}$ NMR (121 MHz, $\left.\mathrm{CDCl}_{3}\right) \delta-1.01$; HRMS (ESI) m/z: $[\mathrm{M}+\mathrm{H}]^{+}$ Calcd. for $\mathrm{C}_{28} \mathrm{H}_{25} \mathrm{NO}_{5} \mathrm{P}$ 486.1470; Found 486.1472.

Note: $2 \mathrm{x}$ aromatic signals not observed in ${ }^{13} \mathrm{C}-\mathrm{NMR}$ spectrum. 


\section{Dibenzyl (4-(methylsulfonyl)benzoyl)phosphoramidate (3r)}<smiles>COS(=O)(=O)c1ccc(C(=O)NP(=O)(Cc2ccccc2)OCc2ccccc2)cc1</smiles>

General procedure A was followed using 4-methylsulfonylbenzoyl azide (0.225 g, $1.0 \mathrm{mmol})$. The reaction mixture was purified using column chromatography gradient eluting from $100 \%$ DCM to $5 \% \mathrm{MeOH} / \mathrm{DCM}$ to give $3 \mathbf{r}$ as a beige solid (412 mg, 90\%). ${ }^{1} \mathrm{H}$ NMR (300 MHz, $\left.\mathrm{CDCl}_{3}\right) \delta 9.82(\mathrm{~d}, J 8.9 \mathrm{~Hz}, 1 \mathrm{H}), 8.23-8.12(\mathrm{~m}, 2 \mathrm{H}), 8.00-7.90(\mathrm{~m}, 2 \mathrm{H}), 7.41-7.24(\mathrm{~m}$, $10 \mathrm{H}), 5.33-5.15(\mathrm{~m}, 4 \mathrm{H}), 3.06(\mathrm{~s}, 3 \mathrm{H}) ;{ }^{13} \mathrm{C} \mathrm{NMR}\left(75 \mathrm{MHz}, \mathrm{CDCl}_{3}\right) \delta 166.6(\mathrm{~d}, J 2.4 \mathrm{~Hz})$, 144.1, 137.2 (d, $J 10.9 \mathrm{~Hz}), 135.4$ (d, $J 6.5 \mathrm{~Hz}), 129.6,128.9,128.8,128.1,127.6,70.3$ (d, $J$ $6.0 \mathrm{~Hz}), 44.4 ;{ }^{31} \mathrm{P}$ NMR $\left(121 \mathrm{MHz}, \mathrm{CDCl}_{3}\right) \delta$-1.54; HRMS (ESI) m/z: $[\mathrm{M}+\mathrm{H}]^{+}$Calcd. For $\mathrm{C}_{22} \mathrm{H}_{23} \mathrm{NO}_{6} \mathrm{PS}$ 460.0984; Found 460.0977.

\section{Dibenzyl (3-(methylcarboxy)benzoyl)phosphoramidate (3s)}<smiles>COC(=O)c1cccc(C(=O)NP(=O)(OCc2ccccc2)OCc2ccccc2)c1</smiles>

General procedure A was followed using 3-(methylcarboxy)benzoyl azide (0.205 g, $1.0 \mathrm{mmol})$. The reaction mixture was purified using column chromatography gradient eluting from $100 \%$ DCM to $5 \% \mathrm{MeOH} / \mathrm{DCM}$ to give $3 \mathrm{~s}$ as a white solid (0.388 g, 88\%). ${ }^{1} \mathrm{H}$ NMR (300 MHz, $\left.\mathrm{CDCl}_{3}\right) \delta 9.61(\mathrm{~d}, J 9.0 \mathrm{~Hz}, 1 \mathrm{H}), 8.74-8.69(\mathrm{~m}, 1 \mathrm{H}), 8.26-8.17(\mathrm{~m}, 2 \mathrm{H}), 7.55-7.46(\mathrm{~m}, 1 \mathrm{H})$, $7.43-7.33(\mathrm{~m}, 4 \mathrm{H}), 7.32-7.21(\mathrm{~m}, 6 \mathrm{H}), 5.34-5.17(\mathrm{~m}, 4 \mathrm{H}), 3.87(\mathrm{~s}, 3 \mathrm{H}) ;{ }^{13} \mathrm{C}$ NMR $(75$ $\left.\mathrm{MHz}, \mathrm{CDCl}_{3}\right) \delta 167.3$ (d, $\left.J 2.6 \mathrm{~Hz}\right), 166.3,135.7$ (d, $\left.J 6.9 \mathrm{~Hz}\right), 133.7,133.0$ (d, $\left.J 10.9 \mathrm{~Hz}\right)$, 132.9, 130.8, 129.6, 128.8, 128.6, 128.6, 128.1, 69.9 (d, J 5.7 Hz), 52.4; ${ }^{31} \mathrm{P}$ NMR (121 MHz, $\left.\mathrm{CDCl}_{3}\right) \delta$-0.99; HRMS (ESI) m/z: [M+H] ${ }^{+}$Calcd. for $\mathrm{C}_{23} \mathrm{H}_{23} \mathrm{NO}_{6} \mathrm{P} 440.1263$; Found 440.1256. 


\section{Dibenzyl (4-methoxybenzoyl)phosphoramidate (3t)}<smiles>COc1ccc(C(=O)NP(=O)(OCc2ccccc2)OCc2ccccc2)cc1</smiles>

General procedure A was followed using 4-methoxybenzoyl azide $(0.177 \mathrm{~g}, 1.0 \mathrm{mmol})$. The reaction mixture was purified using column chromatography gradient eluting from $100 \%$ DCM to $5 \% \mathrm{MeOH} / \mathrm{DCM}$ to give $3 \mathrm{t}$ as a white solid (0.376 g, 91\%). ${ }^{1} \mathrm{H} \mathrm{NMR}\left(300 \mathrm{MHz}, \mathrm{CDCl}_{3}\right) \delta$ $9.04(\mathrm{~d}, J 5.9 \mathrm{~Hz}, 1 \mathrm{H}), 8.01-7.93(\mathrm{~m}, 2 \mathrm{H}), 7.43-7.34(\mathrm{~m}, 4 \mathrm{H}), 7.34-7.24(\mathrm{~m}, 6 \mathrm{H}), 6.92-$ $6.84(\mathrm{~m}, 2 \mathrm{H}), 5.32-5.14(\mathrm{~m}, 4 \mathrm{H}), 3.85(\mathrm{~s}, 3 \mathrm{H}) ;{ }^{13} \mathrm{C} \mathrm{NMR}\left(75 \mathrm{MHz}, \mathrm{CDCl}_{3}\right) \delta 167.5(\mathrm{~d}, J 2.6$ Hz), 163.4, 135.9 (d, J 7.0 Hz), 130.5, 128.6, 128.5, 128.1, 124.9 (d, J 10.7 Hz), 113.9, 69.7 (d, J 5.7 Hz), 55.6; ${ }^{31} \mathrm{P}$ NMR (121 MHz, $\left.\mathrm{CDCl}_{3}\right) \delta-0.32$; HRMS (ESI) m/z: [M+H] ${ }^{+}$Calcd. for $\mathrm{C}_{22} \mathrm{H}_{23} \mathrm{NO}_{5} \mathrm{P}$ 412.1314; Found 412.1307.

\section{Dibenzyl (2-fluorobenzoyl)phosphoramidate (3u)}<smiles>O=C(NP(=O)(OCc1ccccc1)OCc1ccccc1)c1ccccc1F</smiles>

General procedure $\mathrm{C}$ was followed using 2-fluorobenzoic acid (0.140 g, $1.0 \mathrm{mmol})$. The reaction mixture was purified using column chromatography gradient eluting from 100\% DCM to $5 \% \mathrm{MeOH} / \mathrm{DCM}$ to give $3 \mathbf{u}$ as a white solid (289 $\mathrm{mg}, 72 \%) .{ }^{1} \mathrm{H} \mathrm{NMR}\left(300 \mathrm{MHz}, \mathrm{CDCl}_{3}\right) \delta$ $8.07-7.96(\mathrm{~m}, 1 \mathrm{H}), 7.89-7.74(\mathrm{~m}, 1 \mathrm{H}), 7.59-7.47(\mathrm{~m}, 1 \mathrm{H}), 7.44-7.20(\mathrm{~m}, 11 \mathrm{H}), 7.17-$ $7.05(\mathrm{~m}, 1 \mathrm{H}), 5.32$ - $5.16(\mathrm{~m}, 4 \mathrm{H}) ;{ }^{13} \mathrm{C} \mathrm{NMR}\left(75 \mathrm{MHz}, \mathrm{CDCl}_{3}\right) \delta 163.8-163.5(\mathrm{~m}), 160.9(\mathrm{~d}$, C-F, $1 J$ C-F 249.7 Hz), 135.6 (d, J 7.0 Hz), 135.0 (d, J 9.5 Hz), 132.6 - 132.1 (m), 128.6 128.6 (m), 128.2, 125.0 (d, J 3.3 Hz), 120.0 - 119.5 (m), 116.4 (d, J 24.5 Hz), 69.8 (d, J 5.6 $\mathrm{Hz}$ ); ${ }^{31} \mathrm{P}$ NMR $\left(121 \mathrm{MHz}, \mathrm{CDCl}_{3}\right) \delta$-2.85; HRMS (ESI) m/z: $[\mathrm{M}+\mathrm{H}]^{+}$Calcd. for $\mathrm{C}_{21} \mathrm{H}_{20} \mathrm{FNO}_{4} \mathrm{P}$ 400.1114; Found 400.1117.

Note: $1 \mathrm{x}$ aromatic signal not observed in ${ }^{13} \mathrm{C}-\mathrm{NMR}$ spectrum. 


\section{Dibenzyl (2-(methylthio)benzoyl)phosphoramidate (3v)}<smiles>COc1ccccc1C(=O)NP(=O)(OCc1ccccc1)OCc1ccccc1</smiles>

General procedure $\mathrm{C}$ was followed using 2-(methylthio)benzoic acid (0.168 g, $1.0 \mathrm{mmol})$. The crude product was purified by column chromatography gradient eluting with $100 \%$ heptane to $100 \%$ EtOAc to give 3v as a pale yellow oil $(0.390 \mathrm{~g}, 91 \%) .{ }^{1} \mathrm{H}$ NMR $\left(300 \mathrm{MHz}, \mathrm{CDCl}_{3}\right) \delta$ 8.65 (d, J 10.1 Hz, 1H), 7.65 (dd, $J 7.8,1.5 \mathrm{~Hz}, 1 \mathrm{H}), 7.47-7.20$ (m, 13H), $7.20-7.10$ (m, 1H), 5.31 - $5.16(\mathrm{~m}, 4 \mathrm{H}), 2.43(\mathrm{~s}, 3 \mathrm{H}) ;{ }^{13} \mathrm{C} \mathrm{NMR}\left(75 \mathrm{MHz}, \mathrm{CDCl}_{3}\right) \delta 168.2$ (d, J2.3 Hz), 139.0, 135.8 (d, J 7.2 Hz), 132.1, 129.7, 128.6, 128.6, 128.2, 127.6, 125.2, 69.9 (d, J 5.6 Hz), 17.03; ${ }^{31} \mathrm{P}$ NMR (121 MHz, $\left.\mathrm{CDCl}_{3}\right) \delta$-1.64; HRMS (ESI) m/z: $[\mathrm{M}+\mathrm{H}]^{+}$Calcd. for $\mathrm{C}_{22} \mathrm{H}_{23} \mathrm{NO}_{4} \mathrm{PS}$ 428.1085; Found 428.1082.

\section{Dibenzyl (2-nitrobenzoyl)phosphoramidate (3w)}<smiles>O=C(NP(=O)(OCc1ccccc1)Oc1ccccc1)c1ccccc1[N+](=O)[O-]</smiles>

General procedure $\mathrm{C}$ was followed using 2-nitrobenzoic acid (0.167 g, $1.0 \mathrm{mmol})$. The crude product was purified by column chromatography gradient eluting with $100 \%$ heptane to $100 \%$ EtOAc to give 3w as a white solid (0.322 g, 76\%). ${ }^{1} \mathrm{H}$ NMR $\left(300 \mathrm{MHz}, \mathrm{CDCl}_{3}\right) \delta 9.36(\mathrm{~d}, J$ $9.8 \mathrm{~Hz}, 1 \mathrm{H}), 8.12-8.01(\mathrm{~m}, 1 \mathrm{H}), 7.62-7.51(\mathrm{~m}, 2 \mathrm{H}), 7.43-7.27(\mathrm{~m}, 11 \mathrm{H}), 5.17(\mathrm{~d}, J 8.3 \mathrm{~Hz}$, $4 \mathrm{H}) ;{ }^{13} \mathrm{C} \mathrm{NMR}\left(75 \mathrm{MHz}, \mathrm{CDCl}_{3}\right) \delta 167.6(\mathrm{~d}, J 2.0 \mathrm{~Hz}), 146.2,135.6(\mathrm{~d}, J 7.3 \mathrm{~Hz}), 133.8,131.9$ (d, $J 10.7 \mathrm{~Hz}), 131.0,128.9,128.7,128.3,124.5,69.9$ (d, $J 5.7 \mathrm{~Hz}) ;{ }^{31} \mathrm{P}$ NMR (121 MHz, $\left.\mathrm{CDCl}_{3}\right) \delta$-2.85; HRMS (ESI) $\mathrm{m} / \mathrm{z}:[\mathrm{M}+\mathrm{H}]^{+}$Calcd. for $\mathrm{C}_{21} \mathrm{H}_{20} \mathrm{~N}_{2} \mathrm{O}_{6} \mathrm{P}$ 427.1059; Found 427.1054 .

Note: 1x aromatic signal not observed in ${ }^{13} \mathrm{C}-\mathrm{NMR}$ spectrum. 


\section{Dibenzyl (4-cyanobenzoyl)phosphoramidate (3x)}<smiles>N#Cc1ccc(C(=O)NP(=O)(OCc2ccccc2)Oc2ccccc2)cc1</smiles>

General procedure $\mathrm{C}$ was followed using 4-cyanobenzoic acid (0.189 g, $1.0 \mathrm{mmol})$. The crude product was purified by column chromatography gradient eluting with $100 \%$ heptane to $100 \%$ EtOAc to give $3 \mathbf{x}$ as a white solid $(0.398 \mathrm{~g}, 95 \%)$. ${ }^{1} \mathrm{H} \mathrm{NMR}\left(300 \mathrm{MHz}, \mathrm{CDCl}_{3}\right) \delta 9.83(\mathrm{~d}, J 8.8$ $\mathrm{Hz}, 1 \mathrm{H}), 8.12-8.02(\mathrm{~m}, 2 \mathrm{H}), 7.69-7.59(\mathrm{~m}, 2 \mathrm{H}), 7.44-7.21(\mathrm{~m}, 10 \mathrm{H}), 5.33-5.13(\mathrm{~m}, 4 \mathrm{H})$; ${ }^{13} \mathrm{C}$ NMR $\left(75 \mathrm{MHz}, \mathrm{CDCl}_{3}\right) \delta 166.6(\mathrm{~d}, J 2.6 \mathrm{~Hz}), 136.2(\mathrm{~d}, J 11.0 \mathrm{~Hz}), 135.4(\mathrm{~d}, J 6.3 \mathrm{~Hz})$, 132.3, 129.2, 128.9, 128.8, 128.1, 118.0, 116.2, 70.3 (d, $J 6.1 \mathrm{~Hz}) ;{ }^{31} \mathrm{P} \mathrm{NMR}\left(121 \mathrm{MHz}, \mathrm{CDCl}_{3}\right)$ $\delta$-1.53; HRMS (ESI) m/z: [M+H] $]^{+}$Calcd. for $\mathrm{C}_{22} \mathrm{H}_{20} \mathrm{~N}_{2} \mathrm{O}_{4} \mathrm{P} 407.1161$; Found 407.1158.

\section{Dibenzyl (4-ethynylbenzoyl)phosphoramidate (3y)}<smiles>C#Cc1ccc(C(=O)NP(=O)(OCc2ccccc2)Oc2ccccc2)cc1</smiles>

General procedure $\mathrm{C}$ was followed using 4-ethynylbenzoic acid $(0.146 \mathrm{~g}, 1.0 \mathrm{mmol})$. The crude product was purified by column chromatography gradient eluting with $100 \%$ heptane to $100 \%$ EtOAc to give $3 \mathbf{y}$ as a pale yellow oil $(0.356 \mathrm{~g}, 88 \%)$ which crystallized after standing for two weeks. ${ }^{1} \mathrm{H}$ NMR $\left(300 \mathrm{MHz}, \mathrm{CDCl}_{3}\right) \delta 9.24(\mathrm{~d}, J 9.2 \mathrm{~Hz}, 1 \mathrm{H}), 7.94(\mathrm{~d}, J 8.0 \mathrm{~Hz}, 2 \mathrm{H}), 7.52(\mathrm{~d}, J$ $8.0 \mathrm{~Hz}, 2 \mathrm{H}), 7.46-7.17(\mathrm{~m}, 10 \mathrm{H}), 5.34-5.14(\mathrm{~m}, 4 \mathrm{H}), 3.27(\mathrm{~s}, 1 \mathrm{H}) ;{ }^{13} \mathrm{C} \mathrm{NMR}(75 \mathrm{MHz}$, $\left.\mathrm{CDCl}_{3}\right) \delta 167.3(\mathrm{~d}, J 2.7 \mathrm{~Hz}), 135.6(\mathrm{~d}, J 6.8 \mathrm{~Hz}), 132.5,132.3,128.7,128.4,128.2,126.8$, 82.8, 80.3, 70.0 (d, $J 5.8 \mathrm{~Hz}) ;{ }^{31} \mathrm{P}$ NMR (121 MHz, $\left.\mathrm{CDCl}_{3}\right) \delta$-1.01; HRMS (ESI) m/z: [M+H] ${ }^{+}$ Calcd. for $\mathrm{C}_{23} \mathrm{H}_{21} \mathrm{NO}_{4} \mathrm{P}$ 406.1208; Found 406.1204.

Note: 1x aromatic signal not observed in ${ }^{13} \mathrm{C}-\mathrm{NMR}$ spectrum. 


\section{Dibenzyl (4-carbamoylbenzoyl)phosphoramidate (3z)}<smiles>NC(=O)c1ccc(C(=O)NP(=O)(OCc2ccccc2)Oc2ccccc2)cc1</smiles>

General procedure $\mathrm{C}$ was followed using 4-carbamoylbenzoic acid (0.165 g, $1.0 \mathrm{mmol})$. The crude product was purified by trituration with $\mathrm{Et}_{2} \mathrm{O}(2 \mathrm{x} 5 \mathrm{~mL})$ due to poor solubility in DCM. ${ }^{1} \mathrm{H}$ NMR (300 MHz, DMSO-d6) $\delta 10.29$ (s, 1H), 8.14 (s, 1H), 8.06 - 7.93 (m, 4H), 7.57 (s, 1H), $7.44-7.28(\mathrm{~m}, 10 \mathrm{H}), 5.24-5.08(\mathrm{~m}, 4 \mathrm{H}) ;{ }^{13} \mathrm{C}$ NMR (75 MHz, DMSO-d6) $\delta 167.8(\mathrm{~d}, J$ $1.8 \mathrm{~Hz}), 167.0,137.7,136.2$ (d, $J 7.3 \mathrm{~Hz}), 134.9$ (d, $J 11.0 \mathrm{~Hz}), 128.4,128.3,128.2,127.8$, 127.4, 68.3 (d, J 5.3 Hz); ${ }^{31} \mathrm{P}$ NMR (121 MHz, DMSO-d6) $\delta 0.33$; HRMS (ESI) m/z: $[\mathrm{M}+\mathrm{H}]^{+}$ Calcd. for $\mathrm{C}_{22} \mathrm{H}_{22} \mathrm{~N}_{2} \mathrm{O}_{5} \mathrm{P} 425.1266$; Found 425.1269.

\section{tert-Butyl ((2S,3R)-1-((bis(benzyloxy)phosphoryl)amino)-3-hydroxy-1-oxobutan-2-} yl)carbamate (3aa)<smiles>CC(C)[C@H](NC(=O)OCc1ccccc1)C(=O)NP(=O)(OCc1ccccc1)OCc1ccccc1</smiles>

General procedure $\mathrm{C}$ was followed using Boc-Thr-OH $(0.219 \mathrm{~g}, 1.0 \mathrm{mmol})$. The crude product was purified by column chromatography gradient eluting with $100 \%$ heptane to $100 \%$ EtOAc to give 3aa as a colourless oil (0.118 g, 25\%). ${ }^{1} \mathrm{H}$ NMR $\left(300 \mathrm{MHz}, \mathrm{CDCl}_{3}\right) \delta 8.40(\mathrm{~d}, J 11.5$ $\mathrm{Hz}, 1 \mathrm{H}), 7.44-7.17(\mathrm{~m}, 10 \mathrm{H}), 5.57(\mathrm{~d}, J 7.9 \mathrm{~Hz}, 1 \mathrm{H}), 5.25-5.03(\mathrm{~m}, 4 \mathrm{H}), 4.36-4.22(\mathrm{~m}$, $1 \mathrm{H}), 4.18-4.06(\mathrm{~m}, 1 \mathrm{H}), 3.20(\mathrm{~s}, 1 \mathrm{H}), 1.43(\mathrm{~s}, 9 \mathrm{H}), 1.13(\mathrm{~d}, J 6.3 \mathrm{~Hz}, 3 \mathrm{H}) ;{ }^{13} \mathrm{C} \mathrm{NMR}(75 \mathrm{MHz}$, $\left.\mathrm{CDCl}_{3}\right) \delta 173.1,156.4,135.5(\mathrm{dd}, J 7.2,4.4 \mathrm{~Hz}), 128.7,128.7,128.2(\mathrm{~d}, J 1.3 \mathrm{~Hz}), 80.7,70.1$ - 69.5 (m), 66.8, 59.7 (d, J 7.3 Hz), 28.4, 18.6; ${ }^{31} \mathrm{P} \mathrm{NMR}\left(121 \mathrm{MHz}, \mathrm{CDCl}_{3}\right) \delta-1.95 ; \mathrm{HRMS}$ (ESI) m/z: $[\mathrm{M}+\mathrm{H}]^{+}$Calcd. for $\mathrm{C}_{23} \mathrm{H}_{32} \mathrm{~N}_{2} \mathrm{O}_{7} \mathrm{P}$ 479.1947; Found 479.1953. 
(S)-tert-Butyl (1-((bis(benzyloxy)phosphoryl)amino)-1-oxo-3-phenylpropan-2yl)carbamate (3ab)<smiles>O=C(OCc1ccccc1)[C@H](Cc1ccccc1)NC(=O)P(=O)(OCc1ccccc1)OCc1ccccc1</smiles>

General procedure $\mathrm{C}$ was followed using Boc-Phe-OH $(0.265 \mathrm{~g}, 1.0 \mathrm{mmol})$. The crude product was purified by column chromatography gradient eluting with $100 \%$ heptane to $100 \%$ EtOAc to give 3ab as a white solid (0.442 g, 84\%). ${ }^{1} \mathrm{H}$ NMR (300 MHz, $\left.\mathrm{CDCl}_{3}\right) \delta 8.44(\mathrm{~d}, J 10.9 \mathrm{~Hz}$, 1H), $7.40-7.27(\mathrm{~m}, 10 \mathrm{H}), 7.24-7.13(\mathrm{~m}, 5 \mathrm{H}), 5.23$ - $4.96(\mathrm{~m}, 5 \mathrm{H}), 4.51-4.31(\mathrm{~m}, 1 \mathrm{H}), 3.07$ $(\mathrm{dd}, J 14.0,5.6 \mathrm{~Hz}, 1 \mathrm{H}), 2.97-2.81(\mathrm{~m}, 1 \mathrm{H}), 1.36(\mathrm{~s}, 9 \mathrm{H}) ;{ }^{13} \mathrm{C}$ NMR $\left(75 \mathrm{MHz}, \mathrm{DMSO}-d_{6}\right) \delta$ 174.4 (d, J 4.4 Hz), 155.6, 137.7, 136.1 (dd, $J$ 8.0, 2.6 Hz), 129.3, 128.4, 128.2, 128.0, 127.9, 127.7, 126.3, 78.2, $68.3-67.7(\mathrm{~m}), 56.8$ (d, J11.7 Hz), 36.3, 28.1; ${ }^{31} \mathrm{P} \mathrm{NMR}\left(121 \mathrm{MHz}, \mathrm{CDCl}_{3}\right)$ $\delta$-2.02; HRMS (ESI) m/z: [M+H $]^{+}$Calcd. for $\mathrm{C}_{28} \mathrm{H}_{34} \mathrm{~N}_{2} \mathrm{O}_{6} \mathrm{P}$ 525.2155; Found 525.2152.

Note: Additional aromatic signal in ${ }^{13} \mathrm{C}-\mathrm{NMR}$ spectrum in the range of $130-125 \mathrm{ppm}$. Attributed to the presence of a doublet which can not be assigned unambiguously.

(S)-tert-Butyl (1-((bis(benzyloxy)phosphoryl)amino)-3-(1H-indol-3-yl)-1-oxopropan-2yl)carbamate (3ac)<smiles>O=C(N[C@@H](Cc1c[nH]c2ccccc12)C(=O)NP(=O)(OCc1ccccc1)OCc1ccccc1)c1ccccc1</smiles>

General procedure $\mathrm{C}$ was followed using Boc-Trp-OH $(0.304 \mathrm{~g}, 1.0 \mathrm{mmol})$. The crude product was purified by column chromatography gradient eluting with $100 \%$ heptane to $100 \%$ EtOAc to give 3ac as a beige solid (0.223, 40\%). ${ }^{1} \mathrm{H}$ NMR $\left(300 \mathrm{MHz}, \mathrm{CDCl}_{3}\right) \delta 8.42(\mathrm{~d}, J 11.0 \mathrm{~Hz}$, $1 \mathrm{H}), 8.22(\mathrm{~s}, 1 \mathrm{H}), 7.64(\mathrm{~d}, J 7.9 \mathrm{~Hz}, 1 \mathrm{H}), 7.38-7.24(\mathrm{~m}, 11 \mathrm{H}), 7.19-7.11(\mathrm{~m}, 1 \mathrm{H}), 7.11-$ $7.02(\mathrm{~m}, 1 \mathrm{H}), 6.95(\mathrm{~s}, 1 \mathrm{H}), 5.39-4.93(\mathrm{~m}, 5 \mathrm{H}), 4.54(\mathrm{~s}, 1 \mathrm{H}), 3.31-3.10(\mathrm{~m}, 2 \mathrm{H}), 1.38(\mathrm{~s}, 9 \mathrm{H})$; ${ }^{13} \mathrm{C} \mathrm{NMR}\left(75 \mathrm{MHz}, \mathrm{CDCl}_{3}\right) \delta 173.9(\mathrm{~d}, J 3.6 \mathrm{~Hz}), 155.6,136.2,135.7$ (d, $\left.J 7.5 \mathrm{~Hz}\right), 128.6$, 128.2, 128.1, 127.6, 123.6, 122.3, 119.8, 118.8, 111.4, 109.7, 80.4, 69.7 (t, J 4.9 Hz), 56.1, 
28.3, 27.6; ${ }^{31} \mathrm{P}$ NMR (121 MHz, $\left.\mathrm{CDCl}_{3}\right) \delta$-1.9; HRMS (ESI) m/z: $[\mathrm{M}+\mathrm{H}]^{+}$Calcd. for $\mathrm{C}_{30} \mathrm{H}_{35} \mathrm{~N}_{3} \mathrm{O}_{6} \mathrm{P}$ 564.2264; Found: 564.2269.

\section{Dibenzyl tridecanoylphosphoramidate (3ad)}<smiles>CCCCC(=O)NP(=O)(OCc1ccccc1)Oc1ccccc1</smiles>

General procedure $\mathrm{C}$ was followed using tridecanoic acid $(0.214 \mathrm{~g}, 1.0 \mathrm{mmol})$. The crude product was purified by column chromatography gradient eluting with $100 \%$ heptane to $100 \%$ EtOAc to give 3ad as a white solid $(0.323 \mathrm{~g}, 68 \%) .{ }^{1} \mathrm{H}$ NMR $\left(300 \mathrm{MHz}, \mathrm{CDCl}_{3}\right) \delta 8.20(\mathrm{~d}, J$ $10.2 \mathrm{~Hz}, 1 \mathrm{H}), 7.42-7.29$ (m, 10H), $5.25-5.07$ (m, 4H), 2.23 (t, J 7.5 Hz, 2H), $1.62-1.48$ $(\mathrm{m}, 2 \mathrm{H}), 1.37-1.14(\mathrm{~m}, 18 \mathrm{H}), 0.93-0.81(\mathrm{~m}, 3 \mathrm{H}) ;{ }^{13} \mathrm{C} \mathrm{NMR}\left(75 \mathrm{MHz}, \mathrm{CDCl}_{3}\right) \delta 175.0(\mathrm{~d}, J$ $3.3 \mathrm{~Hz}), 135.7$ (d, J 7.0 Hz), 128.7, 128.7, 128.1, 69.7 (d, J 5.7 Hz), 36.9 (d, J 8.4 Hz), 32.1, 29.8, 29.8, 29.8, 29.6, 29.5, 29.2, 24.8, 22.8, 14.2; ${ }^{31} \mathrm{P} \mathrm{NMR}\left(121 \mathrm{MHz}, \mathrm{CDCl}_{3}\right) \delta$-1.34; HRMS (ESI) m/z: $[\mathrm{M}+\mathrm{H}]^{+}$Calcd. for $\mathrm{C}_{27} \mathrm{H}_{41} \mathrm{NO}_{4} \mathrm{P}$ 474.2773; Found 474.2772.

\section{Dibenzyl (2-methyl-2-phenylpropanoyl)phosphoramidate (3ae)}

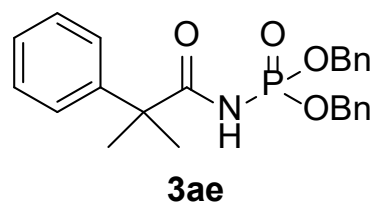

General procedure $\mathrm{C}$ was followed using 2-methyl-2-phenyl-propanoic acid $(0.164 \mathrm{~g}, 1.0$ $\mathrm{mmol}$ ). The crude product was purified by column chromatography gradient eluting with $100 \%$ heptane to $100 \%$ EtOAc to give 3ae as a white solid (0.398 g, 94\%). ${ }^{1} \mathrm{H}$ NMR (300 MHz, $\left.\mathrm{CDCl}_{3}\right) \delta 7.42-7.16(\mathrm{~m}, 15 \mathrm{H}), 6.43(\mathrm{~d}, J 10.9 \mathrm{~Hz}, 1 \mathrm{H}), 5.17-5.02(\mathrm{~m}, 4 \mathrm{H}), 1.49(\mathrm{~s}, 6 \mathrm{H}) ;{ }^{13} \mathrm{C}$ NMR (75 MHz, $\left.\mathrm{CDCl}_{3}\right) \delta 178.16(\mathrm{~d}, J 4.5 \mathrm{~Hz}), 143.44,135.81$ (d, $\left.J 6.9 \mathrm{~Hz}\right), 129.13,128.67$, 128.59, 127.96, 127.68, 126.17, 69.48 (d, $J 5.6 \mathrm{~Hz}), 48.44$ (d, $J 8.3 \mathrm{~Hz}), 26.41 ;{ }^{31} \mathrm{P}$ NMR (121 $\left.\mathrm{MHz}, \mathrm{CDCl}_{3}\right) \delta$-1.24; HRMS (ESI) m/z: $[\mathrm{M}+\mathrm{H}]^{+}$Calcd. for $\mathrm{C}_{24} \mathrm{H}_{27} \mathrm{NO}_{4} \mathrm{P}$ 424.1678; Found 424.1676 . 
<smiles>O=C(NP(=O)(OCc1ccccc1)OCc1ccccc1)c1ccccc1</smiles>

General procedure D was followed using benzhydrazide $(0.136 \mathrm{~g}, 1.0 \mathrm{mmol})$. The crude product was purified by column chromatography gradient eluting with $100 \%$ heptane to $100 \%$ EtOAc to give 3a as a white solid $(0.317 \mathrm{~g}, 83 \%)$. Spectral data matches compound synthesised from acyl azide using general procedure A.

(S)-tert-Butyl (1-((bis(benzyloxy)phosphoryl)amino)-1-oxo-3-phenylpropan-2yl)carbamate (3ab) - from hydrazide<smiles>O=C(N[C@@H](Cc1ccccc1)C(=O)NP(=O)(OCc1ccccc1)OCc1ccccc1)Oc1ccccc1</smiles>

General procedure D was followed using Boc-Phe- $\mathrm{NHNH}_{2}(0.279 \mathrm{~g}, 1.0 \mathrm{mmol})$. The crude product was purified by column chromatography gradient eluting with $100 \%$ heptane to $100 \%$ EtOAc to give $\mathbf{3 a b}$ as a white solid $(0.423 \mathrm{~g}, 81 \%)$. Spectral data matches compound synthesised from carboxylic acid using general procedure $\mathrm{C}$.

\section{Dibenzyl furan-2-carbonylphosphoramidate (3h) - from hydrazide}<smiles>O=C(NP(=O)(OCc1ccccc1)OCc1ccccc1)c1ccco1</smiles>

$3 \mathrm{~h}$

General procedure D was followed using 2-furoic hydrazide $(0.126 \mathrm{~g}, 1.0 \mathrm{mmol})$. The crude product was purified by column chromatography gradient eluting with $100 \%$ heptane to $100 \%$ EtOAc to give $3 \mathrm{~h}$ as a beige solid $(0.301 \mathrm{~g}, 81 \%)$. Spectral data matches compound synthesised from carboxylic acid using general procedure $\mathrm{C}$. 
tert-Butyl-N-[3-[[(3aR,4R,6R,6aR)-4-(6-aminopurin-9-yl)-2,2-dimethyl-3a,4,6,6atetrahydrofuro[3,4-d][1,3] dioxol-6-yl]methoxyphosphonoyloxy]propyl]carbamate (9)<smiles>[2H]P(=O)(OCCCNC(=O)OC(C)(C)C)OCC1[C@@H]2OC(C)(C)[C@H]2O[C@@H]1n1cnc2c(N)ncnc21</smiles>

2',3'-O-Isopropylidene adenosine 8 (3.07 g, $10.0 \mathrm{mmol})$ was dissolved in pyridine $(20 \mathrm{~mL})$ with heating to $60{ }^{\circ} \mathrm{C}$. The solution was then cooled to room temperature and diphenyl phosphite $(75 \%)(4.00 \mathrm{~mL}, 15.8 \mathrm{mmol})$ was added and the solution stirred for $2 \mathrm{~h}$ at room temperature. 3-(Boc-amino) propanol $(3.5 \mathrm{~mL}, 20 \mathrm{mmol})$ was then added, and the mixture stirred for a further $3 \mathrm{~h}$ at room temperature. The reaction mixture was diluted in EtOAc (200 $\mathrm{mL})$ and washed with water $(4 \times 100 \mathrm{~mL})$ and brine $(20 \mathrm{~mL})$. The organic layer was then dried over $\mathrm{MgSO}_{4}$, filtered and concentrated. The residue was purified by column chromatography gradient eluting with $100 \%$ DCM to $50 \%$ EtOAc/DCM, then $100 \%$ DCM to $5 \% \mathrm{MeOH} / \mathrm{DCM}$ to give a P-epimeric mixture of 9 as a colourless residue $(4.055 \mathrm{~g}, 77 \%) .{ }^{1} \mathrm{H}$ NMR $(300 \mathrm{MHz}$, MeOD) $\delta 8.6-8.5(\mathrm{~m}, 1 \mathrm{H}), 8.4(\mathrm{~s}, 1 \mathrm{H}), 6.2-6.1(\mathrm{~m}, 1 \mathrm{H}), 4.7-4.6(\mathrm{~m}, 1 \mathrm{H}), 4.6-4.2(\mathrm{~m}, 7 \mathrm{H})$, $3.2-3.0(\mathrm{~m}, 2 \mathrm{H}), 2.2-2.0(\mathrm{~m}, 2 \mathrm{H}) ;{ }^{13} \mathrm{C} \mathrm{NMR}(75 \mathrm{MHz}, \mathrm{MeOD}) \delta 158.4,157.0(\mathrm{~d}, J 4.9 \mathrm{~Hz})$, 153.4 (d, J6.5 Hz), 150.2, 142.0 (d, J5.0 Hz), 120.6, 115.7, 91.7 (d, J3.0 Hz), $86.9-86.3$ (m), $85.3(\mathrm{~d}, J 7.6 \mathrm{~Hz}), 82.5,66.8$ - $66.4(\mathrm{~m}), 64.8$ (d, J 5.6 Hz), 37.5, 31.7, 28.8, 27.4, 25.5; ${ }^{31} \mathrm{P}$ NMR (121 MHz, $\left.\mathrm{CDCl}_{3}\right) \delta$ 9.5, 9.3; HRMS (ESI) m/z: $[\mathrm{M}+\mathrm{H}]^{+}$Calcd. for $\mathrm{C}_{21} \mathrm{H}_{34} \mathrm{~N}_{6} \mathrm{O} 8 \mathrm{P}$ 529.2176; Found 529.2176.

Note: Note: PH signal observed at $7.93 \mathrm{ppm}$ and $5.53 \mathrm{ppm}\left(J_{\mathrm{PH}} 721 \mathrm{~Hz}\right)$, integrated as separate multiplets. 
(3S)-tert-Butyl 4-(((((3aR,4R,6R,6aR)-6-(6-amino-9H-purin-9-yl)-2,2-

dimethyltetrahydrofuro[3,4-d][1,3] dioxol-4-yl)methoxy)(3-((tert-

butoxycarbonyl)amino)propoxy)phosphoryl)amino)-3-((tert-butoxycarbonyl)amino)-4oxobutanoate (10)

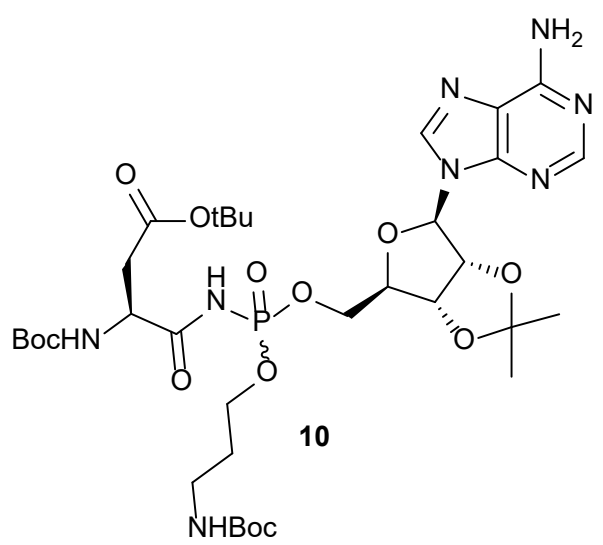

A solution of Boc-L-Asp(OtBu)-OH $(640 \mathrm{mg}, 2.2 \mathrm{mmol})$ and triethylamine $(1.12 \mathrm{~mL}, 8.00$ $\mathrm{mmol})$ in DMF $(5 \mathrm{~mL})$ was cooled on ice and DPPA $(0.43 \mathrm{~mL}, 2.0 \mathrm{mmol})$ was added. The reaction mixture was stirred for $1 \mathrm{~h}$ at $0{ }^{\circ} \mathrm{C}$. The reaction mixture was then removed from the ice bath. After complete consumption of DPPA, the mixture was diluted with DCM $(20 \mathrm{~mL})$ and 9 (529 mg, $1.00 \mathrm{mmol})$ was added followed by TMS-Cl $(0.84 \mathrm{~mL}, 6.6 \mathrm{mmol})$. The reaction mixture was stirred at room temperature for $18 \mathrm{~h}$. The reaction mixture was concentrated then diluted with EtOAc $(100 \mathrm{~mL})$ then washed successively with water $(3 \times 100 \mathrm{~mL})$, saturated $\mathrm{NaHCO}_{3}(2 \times 20 \mathrm{~mL})$ and brine $(50 \mathrm{~mL})$. The organic layer was then dried over $\mathrm{MgSO}_{4}$, filtered and concentrated. The residue was then purified by column chromatography gradient eluting with $100 \%$ DCM to $50 \%$ EtOAc/DCM, then $100 \%$ DCM to $5 \% \mathrm{MeOH} / \mathrm{DCM}$ to give $\mathbf{1 0}$ as a colourless residue (518 mg, 64\% yield). ${ }^{1} \mathrm{H}$ NMR (300 MHz, MeOD) $\delta 8.4-8.3$ (m, 1H), 8.2 $(\mathrm{s}, 1 \mathrm{H}), 6.3-6.2(\mathrm{~m}, 1 \mathrm{H}), 5.4-5.4(\mathrm{~m}, 1 \mathrm{H}), 5.2-5.1(\mathrm{~m}, 1 \mathrm{H}), 4.5-4.3(\mathrm{~m}, 4 \mathrm{H}), 4.2-4.0(\mathrm{~m}$, $2 \mathrm{H}), 3.2-3.0(\mathrm{~m}, 2 \mathrm{H}), 2.8-2.7(\mathrm{~m}, 1 \mathrm{H}), 2.6-2.5(\mathrm{~m}, 1 \mathrm{H}), 1.8-1.7(\mathrm{~m}, 2 \mathrm{H}), 1.6(\mathrm{~s}, 3 \mathrm{H}), 1.5$ - $1.4(\mathrm{~m}, 30 \mathrm{H}) ;{ }^{13} \mathrm{C}$ NMR (75 MHz, MeOD) $\delta 175.2,171.1,158.3,157.4,156.9,153.5,150.2$ (d, J 5.0 Hz), 141.5, 120.4, 115.7 - 114.9 (m), 92.0 - 91.6 (m), 86.3 (d, J 7.0 Hz), 85.5, 82.9 $82.6(\mathrm{~m}), 82.5,81.0,80.0,68.5,66.8,53.7$ - $52.8(\mathrm{~m}), 37.9,37.6,31.4$ (d, J 6.0 Hz), 28.8, 28.7, 28.3, 27.5, 25.6 (d, $J 1.8 \mathrm{~Hz}$ ); ${ }^{31} \mathrm{P}$ NMR (121 MHz, MeOD) $\delta-0.4,-0.7$; HRMS (ESI) m/z: $[\mathrm{M}+\mathrm{H}]^{+}$Calcd. for $\mathrm{C}_{34} \mathrm{H}_{56} \mathrm{~N}_{8} \mathrm{O}_{13} \mathrm{P}$ 815.3705; Found 815.3704. 
(3S)-3-Amino-4-(((((2R,3S,4R,5R)-5-(6-amino-9H-purin-9-yl)-3,4-

dihydroxytetrahydrofuran-2-yl)methoxy)(3-aminopropoxy)phosphoryl)amino)-4oxobutanoic acid (11)<smiles>NCCCOP(=O)(NC(=O)[C@@H](N)CC(=O)O)OCC1O[C@@H](n2cnc3c(N)ncnc32)[C@H](O)[C@@H]1O</smiles>

10 (322 mg, $0.395 \mathrm{mmol})$ was dissolved in TFA $(5 \mathrm{~mL})$ at room temperature for $3 \mathrm{~h}$. The reaction mixture was then concentrated, and the residue purified by HILIC chromatography as follows. The sample was dissolved in water $(0.5 \mathrm{~mL})$ then diluted with $\mathrm{MeCN}(5 \mathrm{~mL})$ and liquid loaded onto a silica column (24 g) equilibrated with 95\% MeCN/water. The column was then eluted with 95 to $70 \% \mathrm{MeCN} /$ water with $0.1 \%$ TFA. The desired fractions were then collected and concentrated to approximated $25 \%$ volume. The solution was then lyophilised to give 11 as a colourless hygroscopic solid (219 mg, 88\% yield). The product was isolated as the TFA salt as confirmed by ${ }^{19} \mathrm{~F}$ NMR. ${ }^{1} \mathrm{H}$ NMR $(300 \mathrm{MHz}, \mathrm{MeOD}) \delta 8.60-8.52(\mathrm{~m}, 1 \mathrm{H}), 8.43$ $(\mathrm{s}, 1 \mathrm{H}), 6.19-6.10(\mathrm{~m}, 1 \mathrm{H}), 4.71-4.60(\mathrm{~m}, 1 \mathrm{H}), 4.59-4.21(\mathrm{~m}, 7 \mathrm{H}), 3.21-2.99(\mathrm{~m}, 2 \mathrm{H})$, 2.16 - $2.00(\mathrm{~m}, 2 \mathrm{H}) ;{ }^{13} \mathrm{C}$ NMR (75 MHz, MeOD) $\delta 172.7$ - $172.4(\mathrm{~m}), 172.0$ - $171.7(\mathrm{~m})$, 152.6, 150.0 (d, J3.9 Hz), 146.6 - 146.2 (m), 143.7 - 143.3 (m), 120.4, 90.5 (d, J9.4 Hz), 84.6 - 83.9 (m), 75.8, 71.5 - $71.0(\mathrm{~m}), 68.8$ - $68.2(\mathrm{~m}), 66.9-66.4(\mathrm{~m}), 51.9$ (d, J 12.8 Hz), 37.8, 35.6 - 35.1 (m), 29.0 (d, J 7.4 Hz); ${ }^{31} \mathrm{P}$ NMR (121 MHz, MeOD) $\delta-1.2(\mathrm{~d}, J 3.2 \mathrm{~Hz}),-1.5$ (d, $J 4.9 \mathrm{~Hz}$ ); ${ }^{19} \mathrm{~F}$ NMR (282 MHz, MeOD) $\delta$-77.1; HRMS (ESI) m/z: $[\mathrm{M}+\mathrm{H}]^{+}$Calcd. for $\mathrm{C}_{17} \mathrm{H}_{28} \mathrm{~N}_{8} \mathrm{O} 9 \mathrm{P}$ 519.1717; Found 519.1711. 


\section{References}

1. Xiao, W.; Zhou, C.-Y.; Che, C.-M. Ruthenium(IV) Porphyrin Catalyzed Phosphoramidation of Aldehydes with Phosphoryl Azides as a Nitrene Source. Chem. Commun. 2012, 48 (47), 5871-5873.

2. He, X.; Ji, Y.; Jin, Y.; Kan, S.; Xia, H.; Chen, J.; Liang, B.; Wu, H.; Guo, K.; Li, Z., Bifunctional imidodiphosphoric acid-catalyzed controlled/living ring-opening polymerization of trimethylene carbonate resulting block, $\alpha, \omega$-dihydroxy telechelic, and star-shaped polycarbonates. Journal of Polymer Science Part A: Polymer Chemistry 2014, 52, 1009-1019. 
NMR spectra and LC chromatograms

Dibenzyl benzoylphosphoramidate (3a)

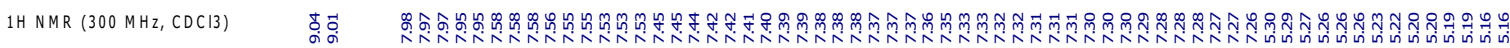

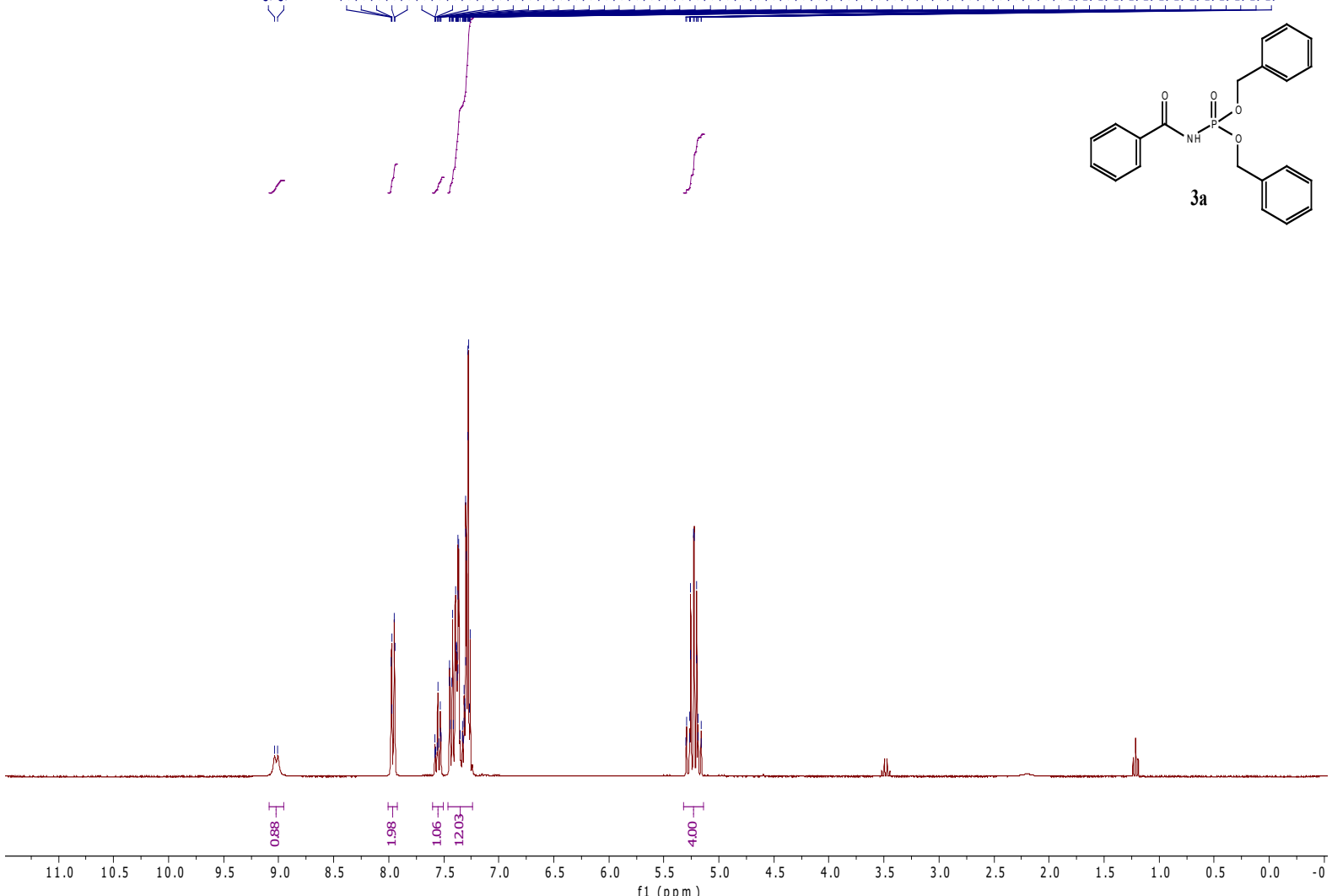

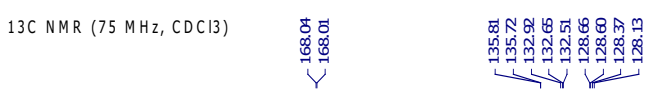

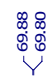
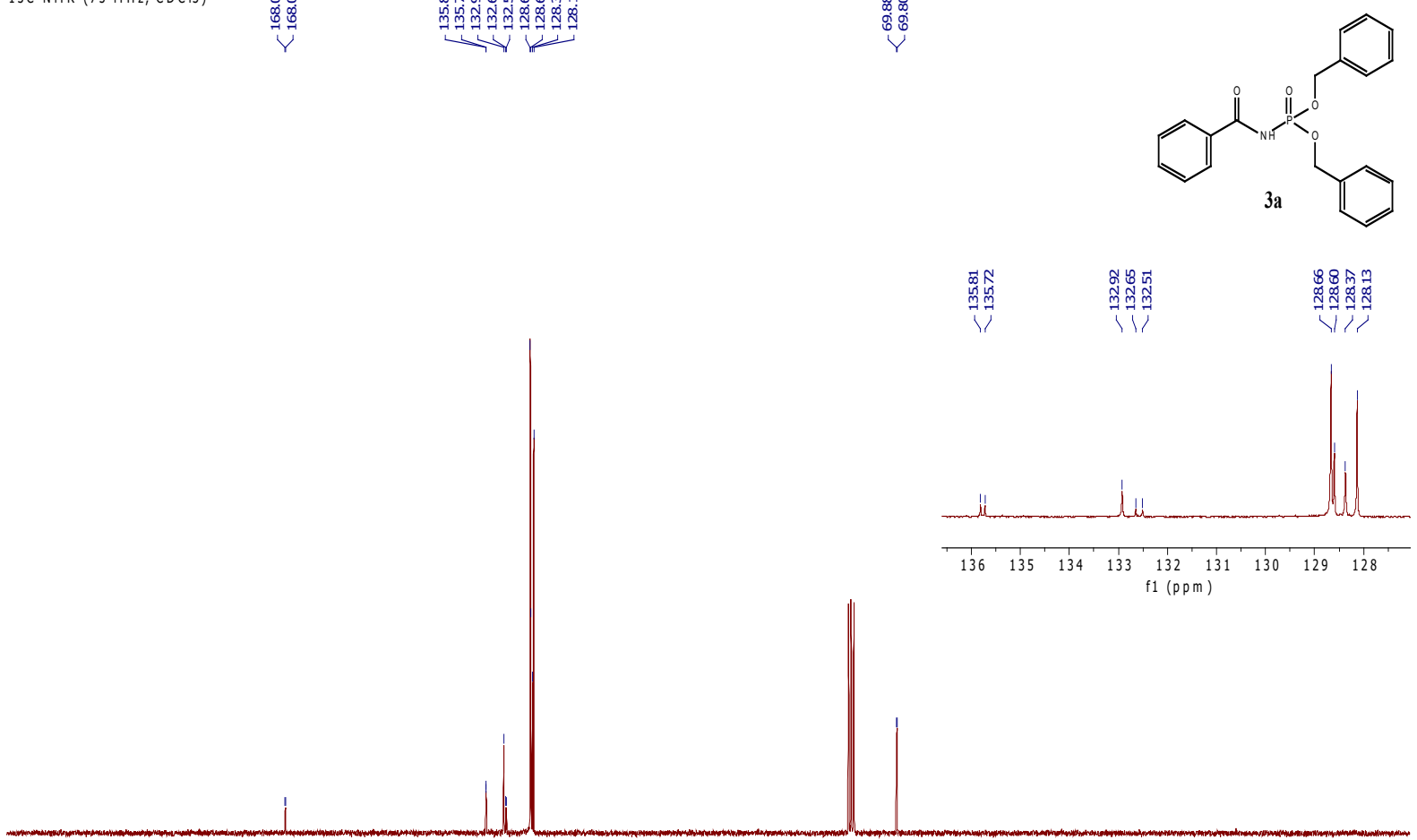

\begin{tabular}{lllllllllllllllllllllllllll}
\hline 210 & 200 & 190 & 180 & 170 & 160 & 150 & 140 & 130 & 120 & 110 & 100 & 90 & 80 & 70 & 60 & 50 & 40 & 30 & 20 & 10 & 0 & -10
\end{tabular} 

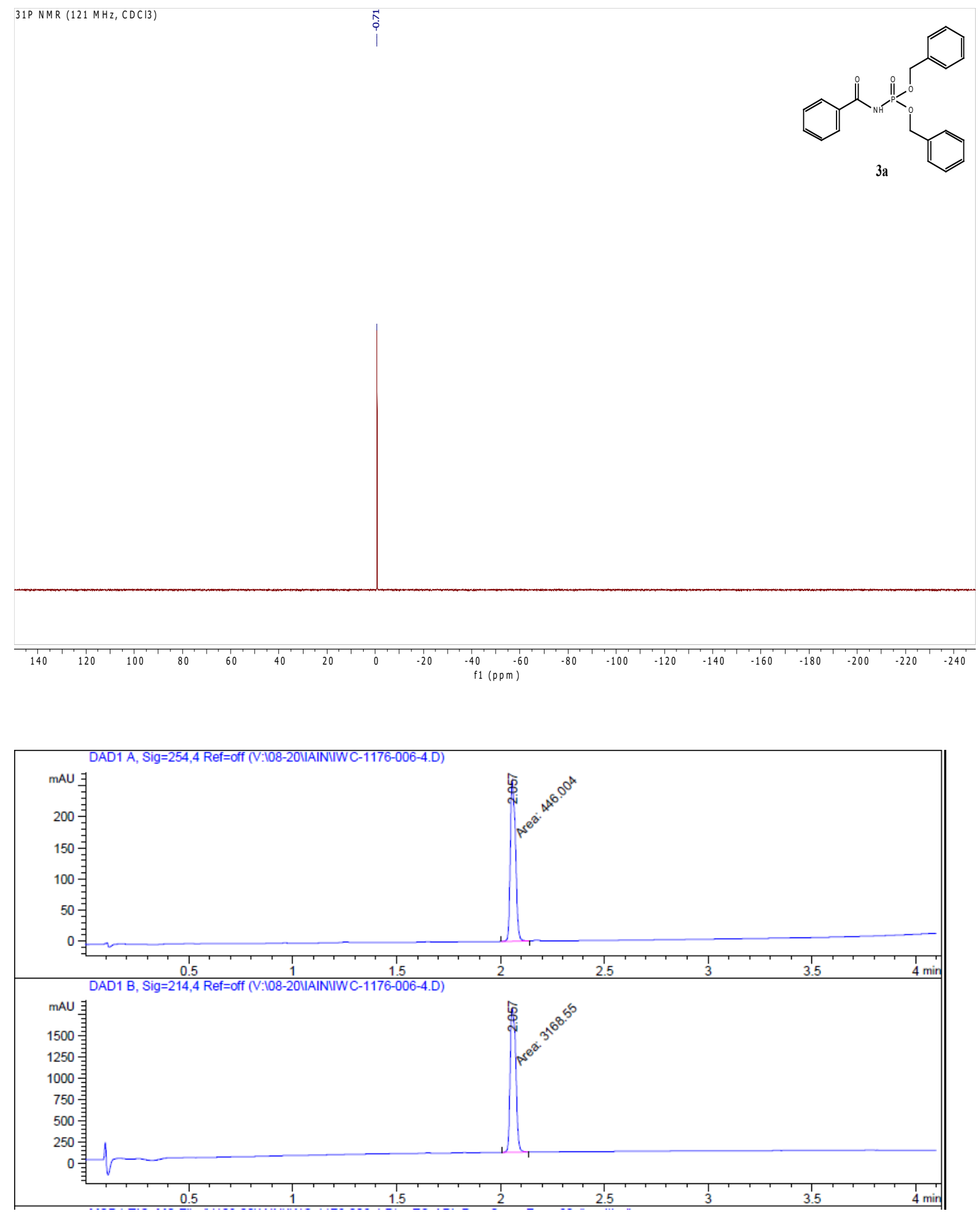
Diphenyl benzoylphosphoramidate (3b)

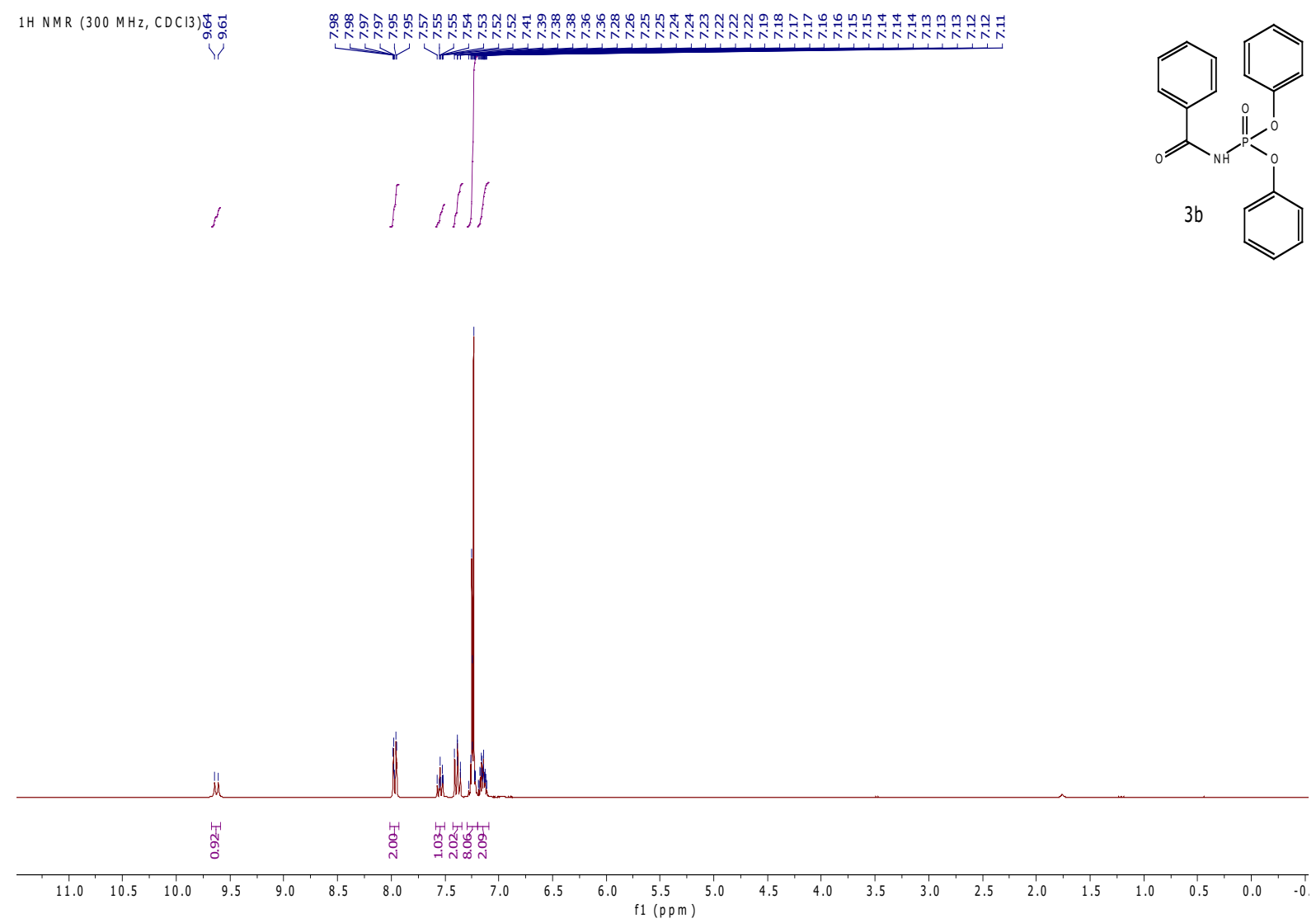

13CNMR (75 MHz, CDCl3)
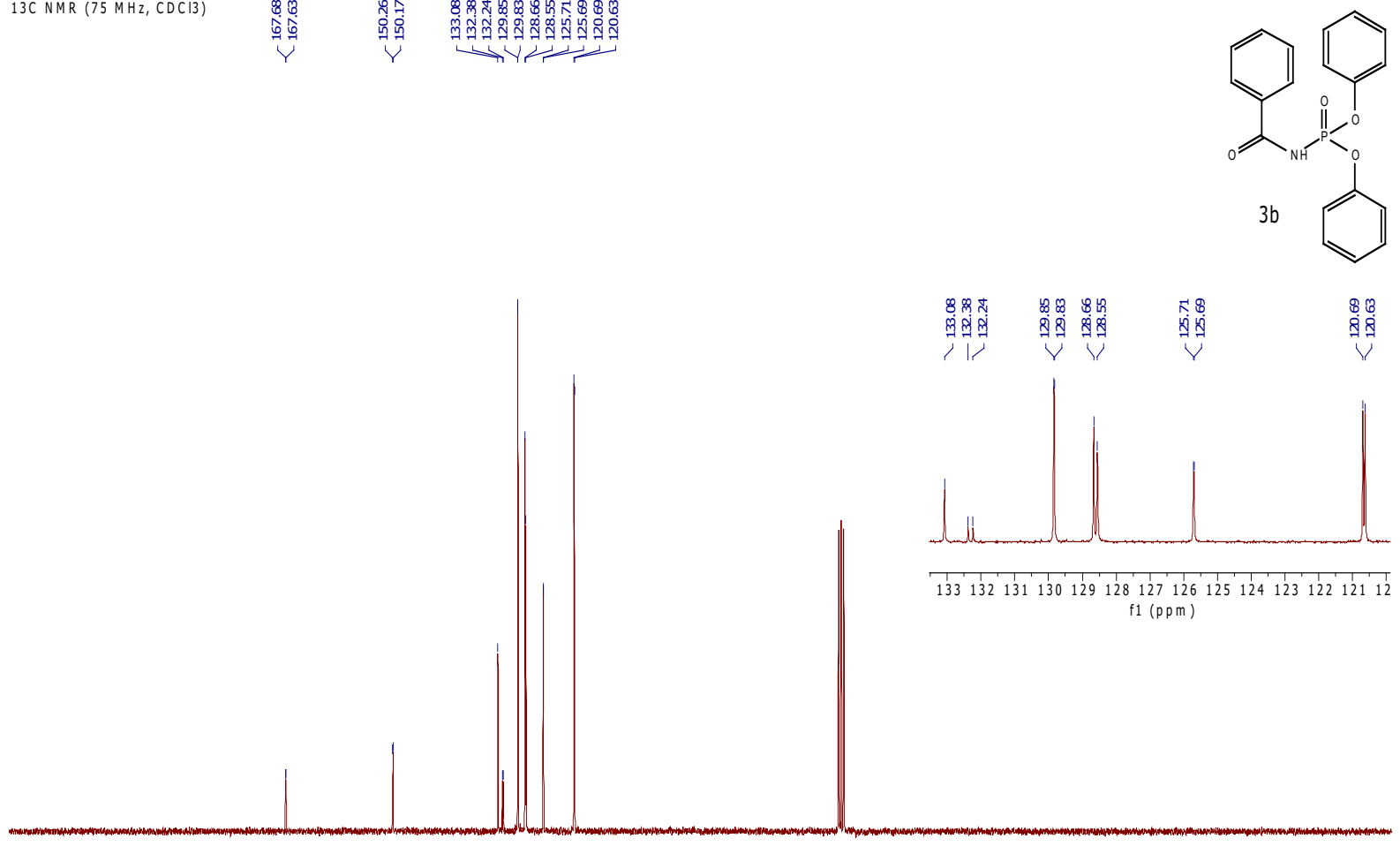

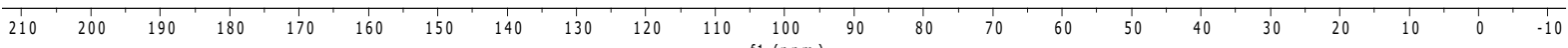



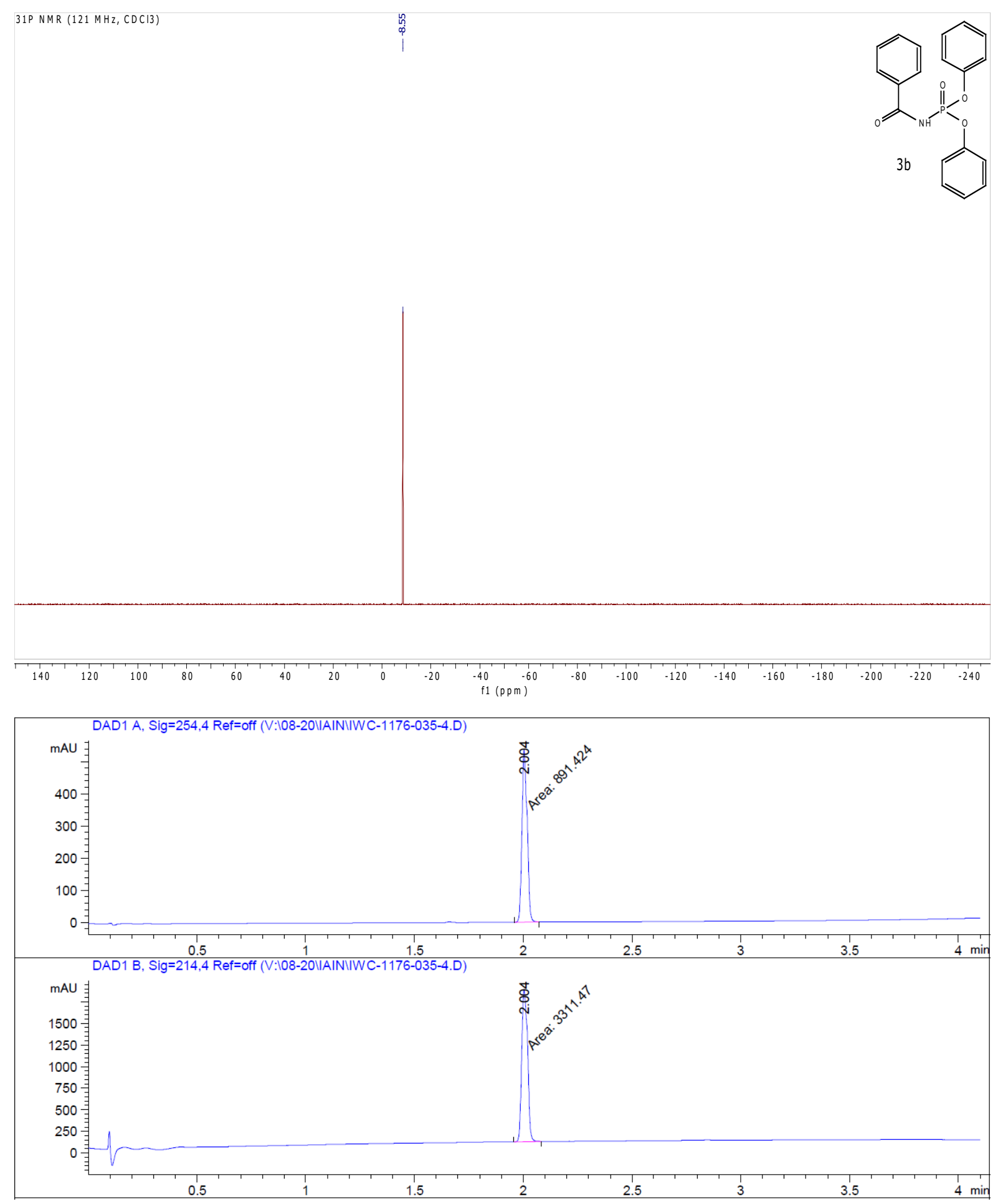
Di-tert-butyl benzoylphosphoramidate (3c)
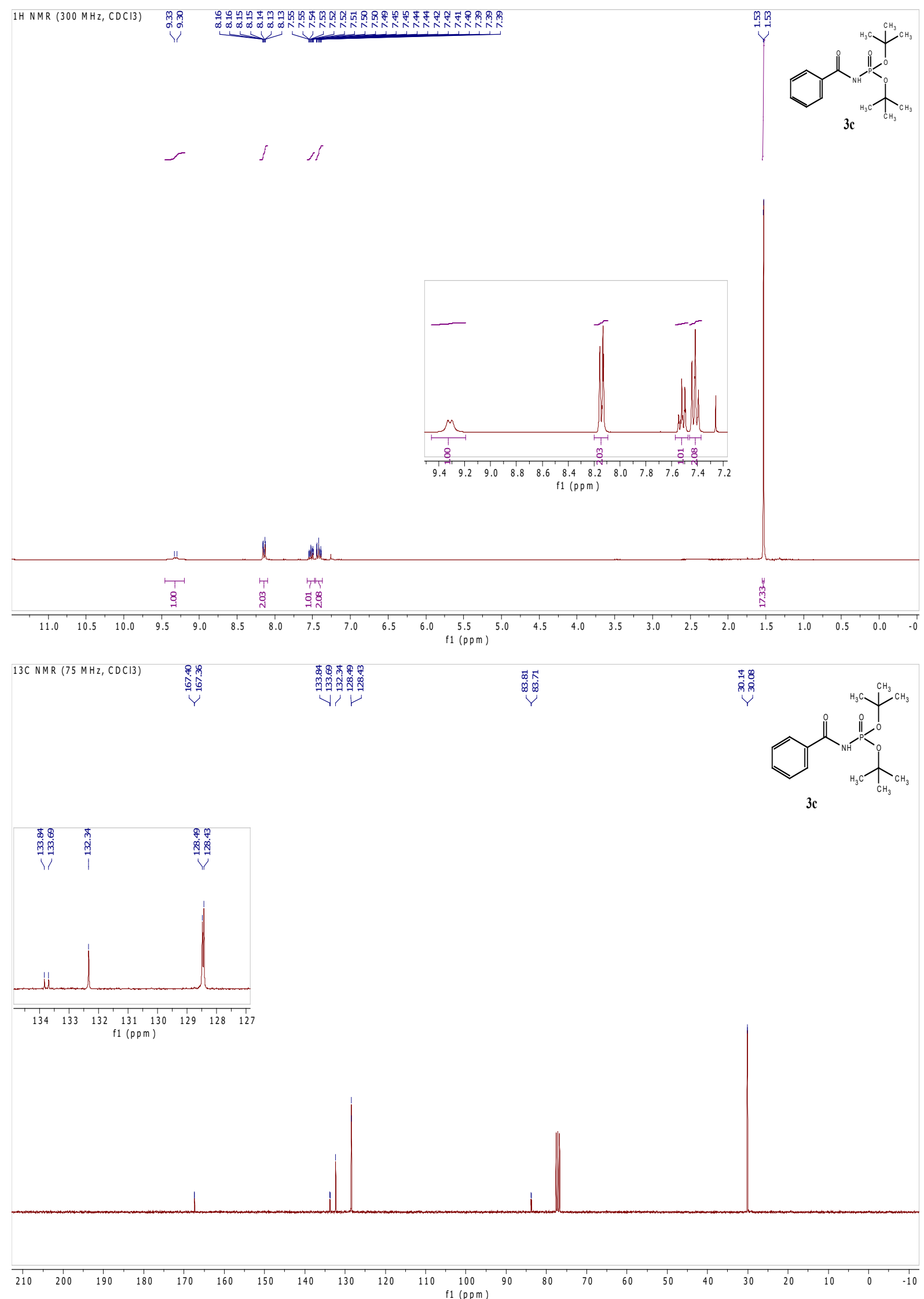


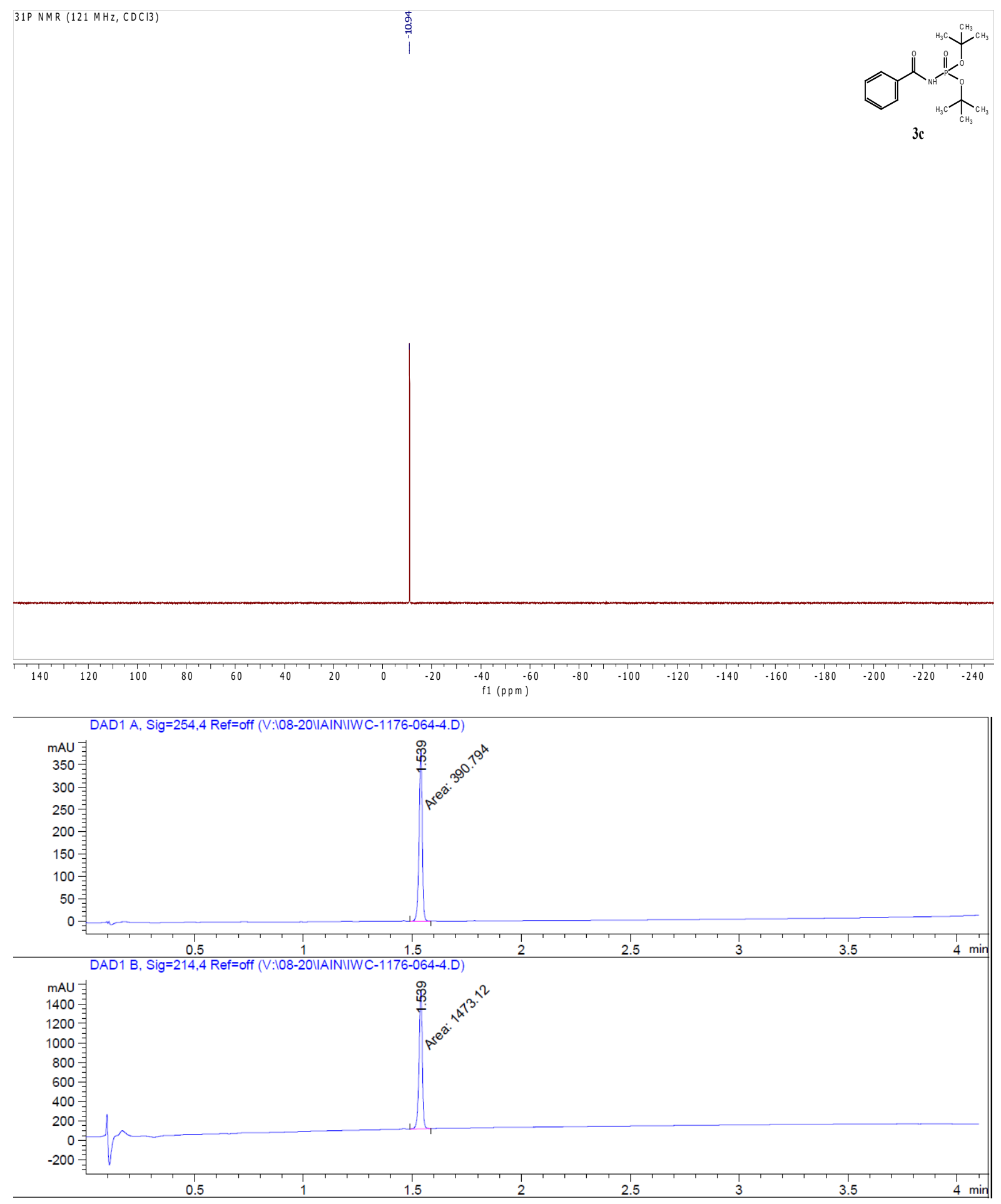


Dimethyl benzoylphosphoramidate (3d)
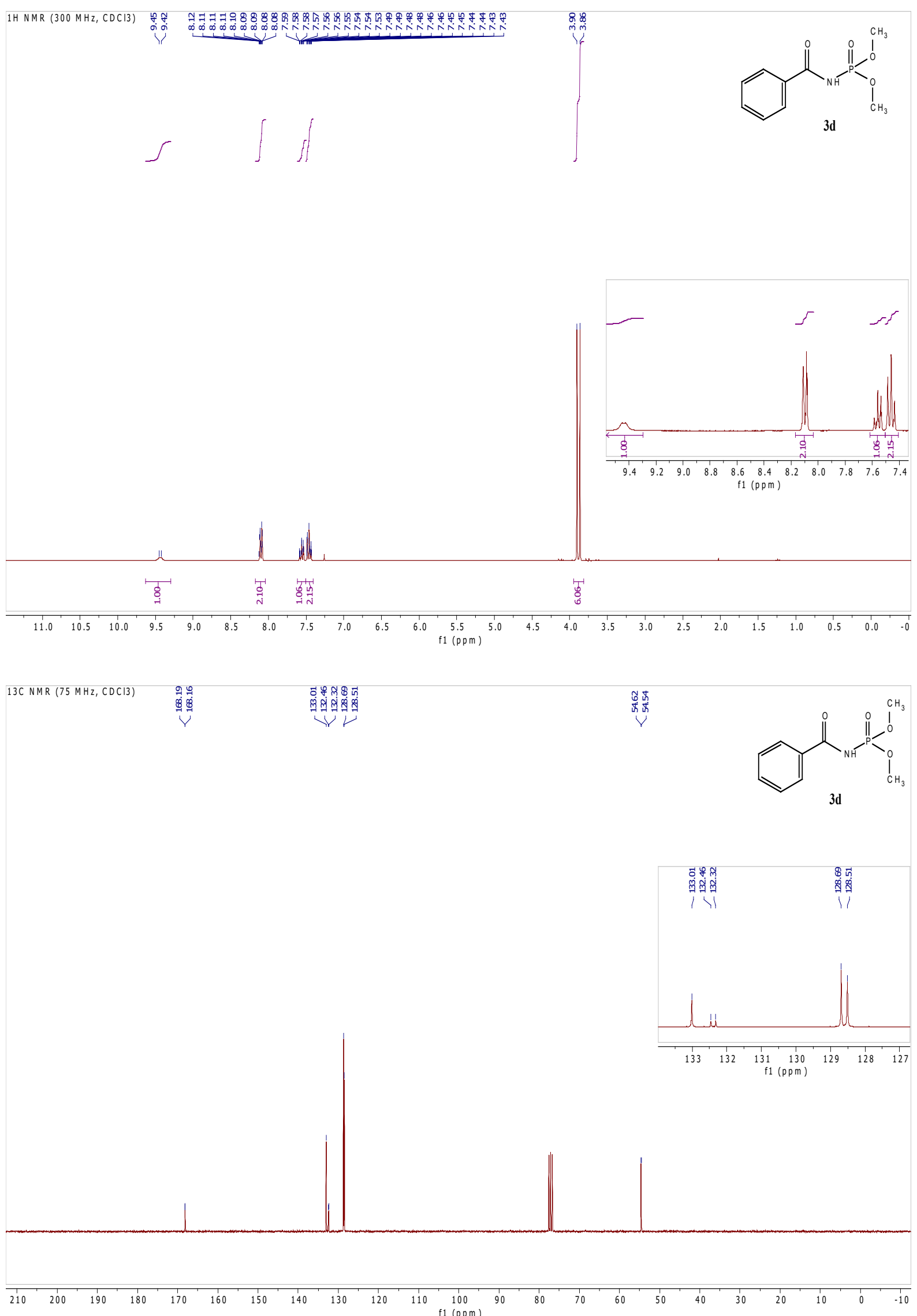

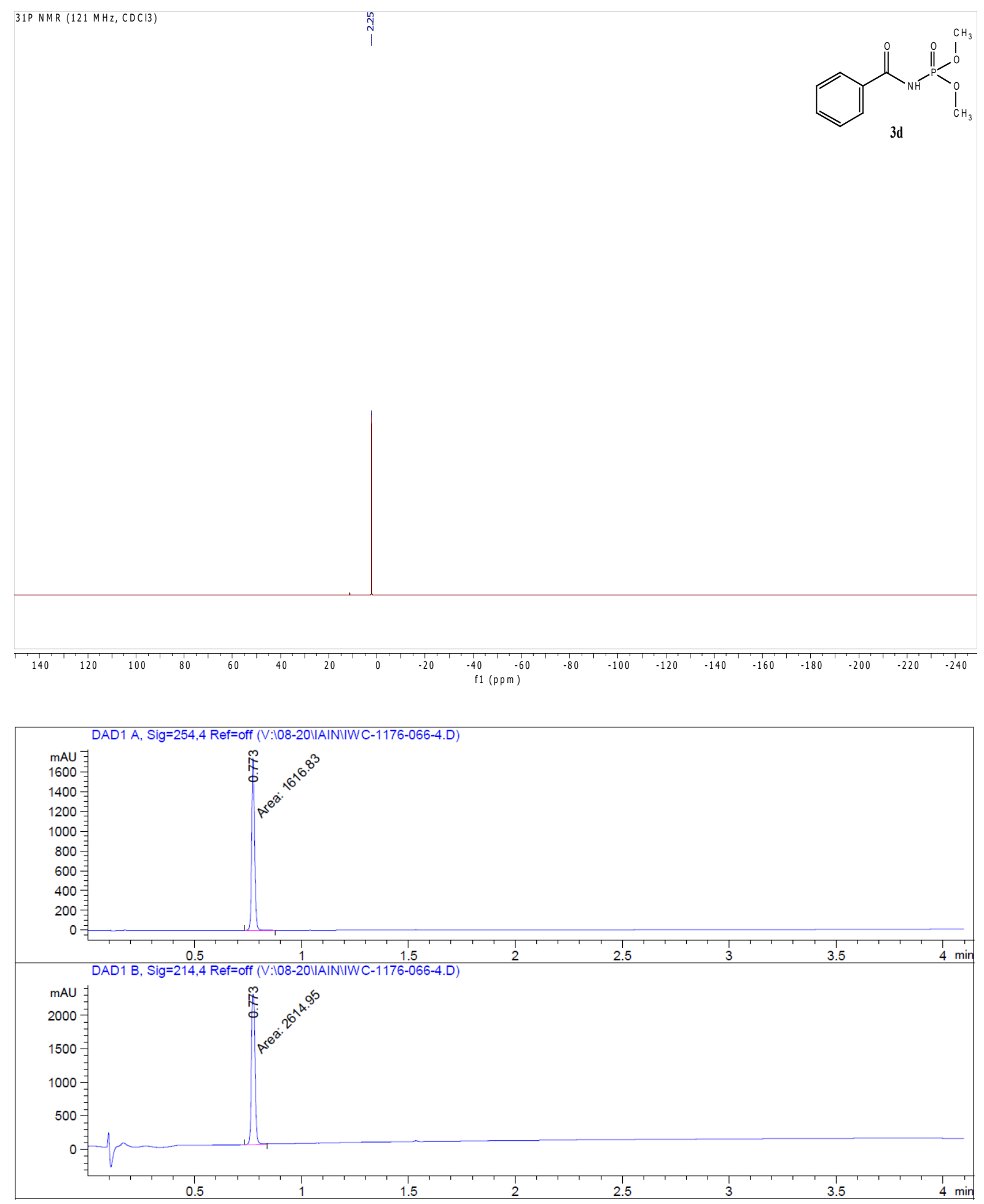


\section{$N$-(Diphenylphosphoryl)benzamide (3e)}

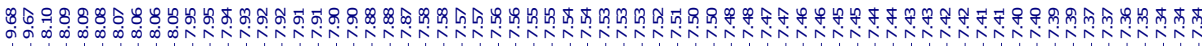

1H NMR $(300 \mathrm{MHz}, \mathrm{CDCl}$ $\begin{array}{cc}5 & 1 \\ 5 & 1\end{array}$
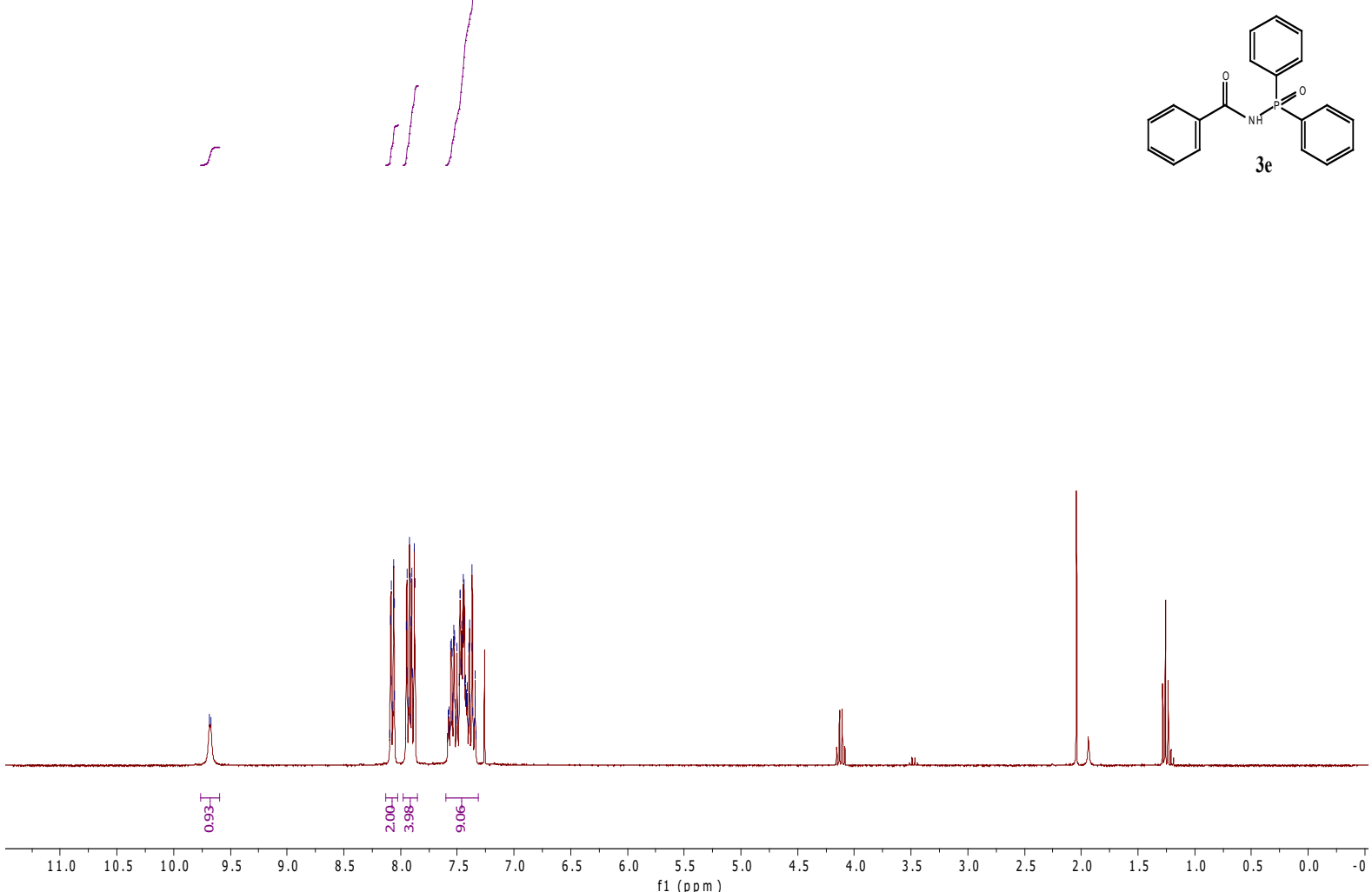

\begin{tabular}{|c|c|}
\hline $13 \mathrm{CNMR}(75 \mathrm{MHz}, \mathrm{CDCl} 3)$ & 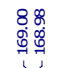 \\
\hline
\end{tabular}
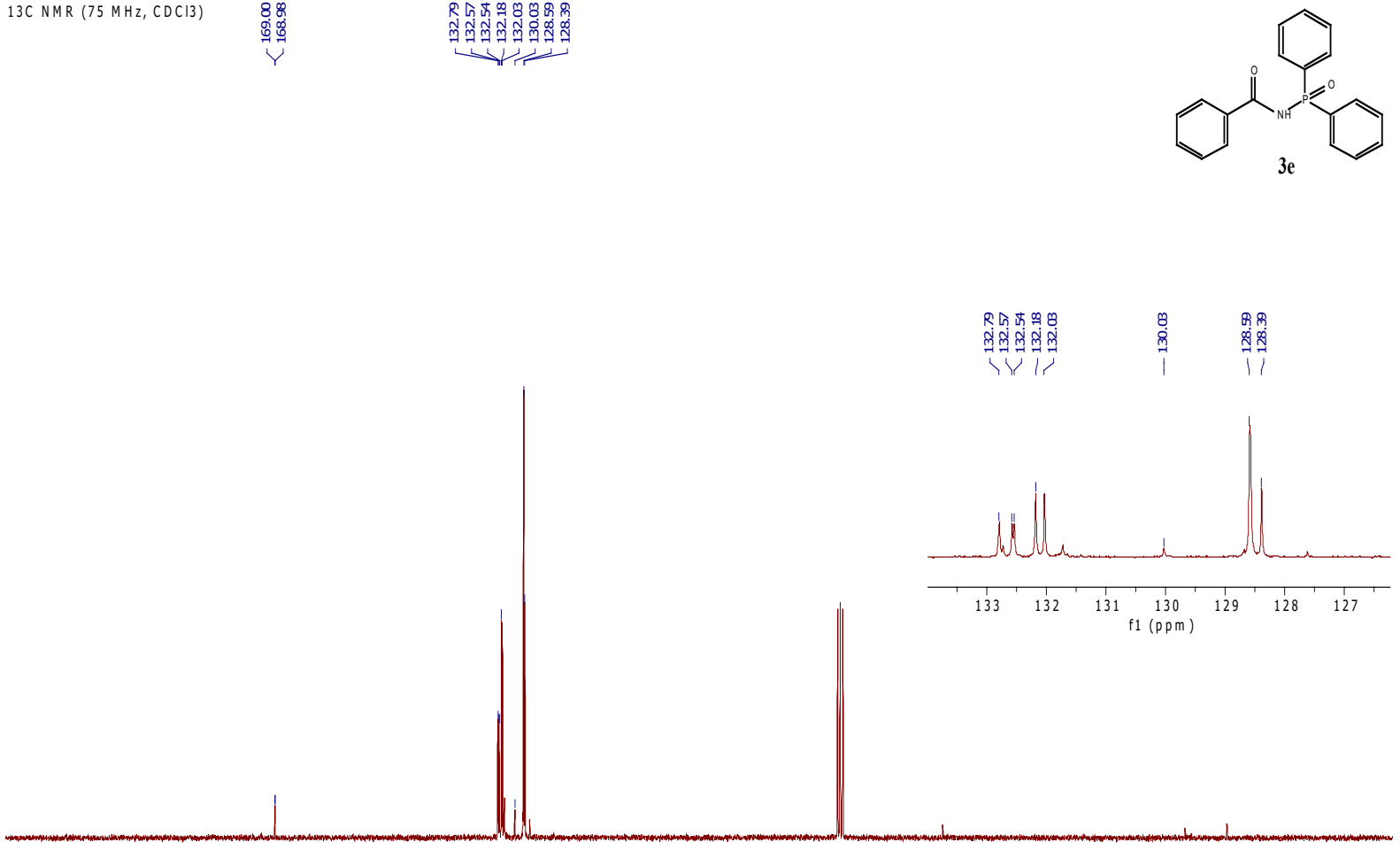

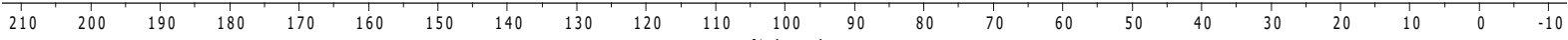


31 P NMR $(121 \mathrm{MHz}, \mathrm{CDCl} 3)$

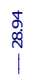

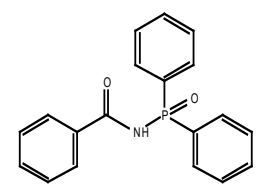

$3 e$

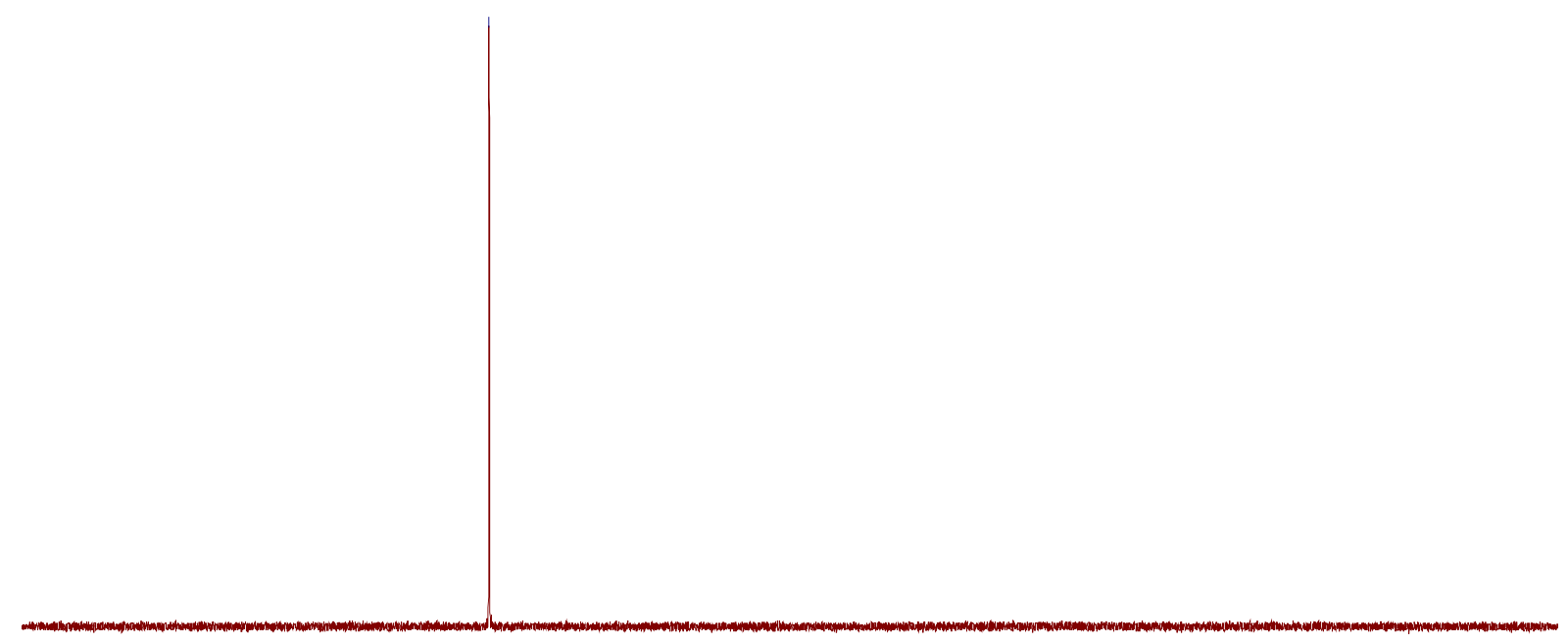

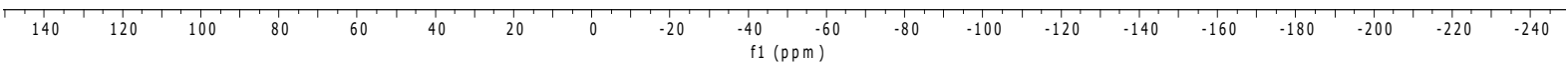

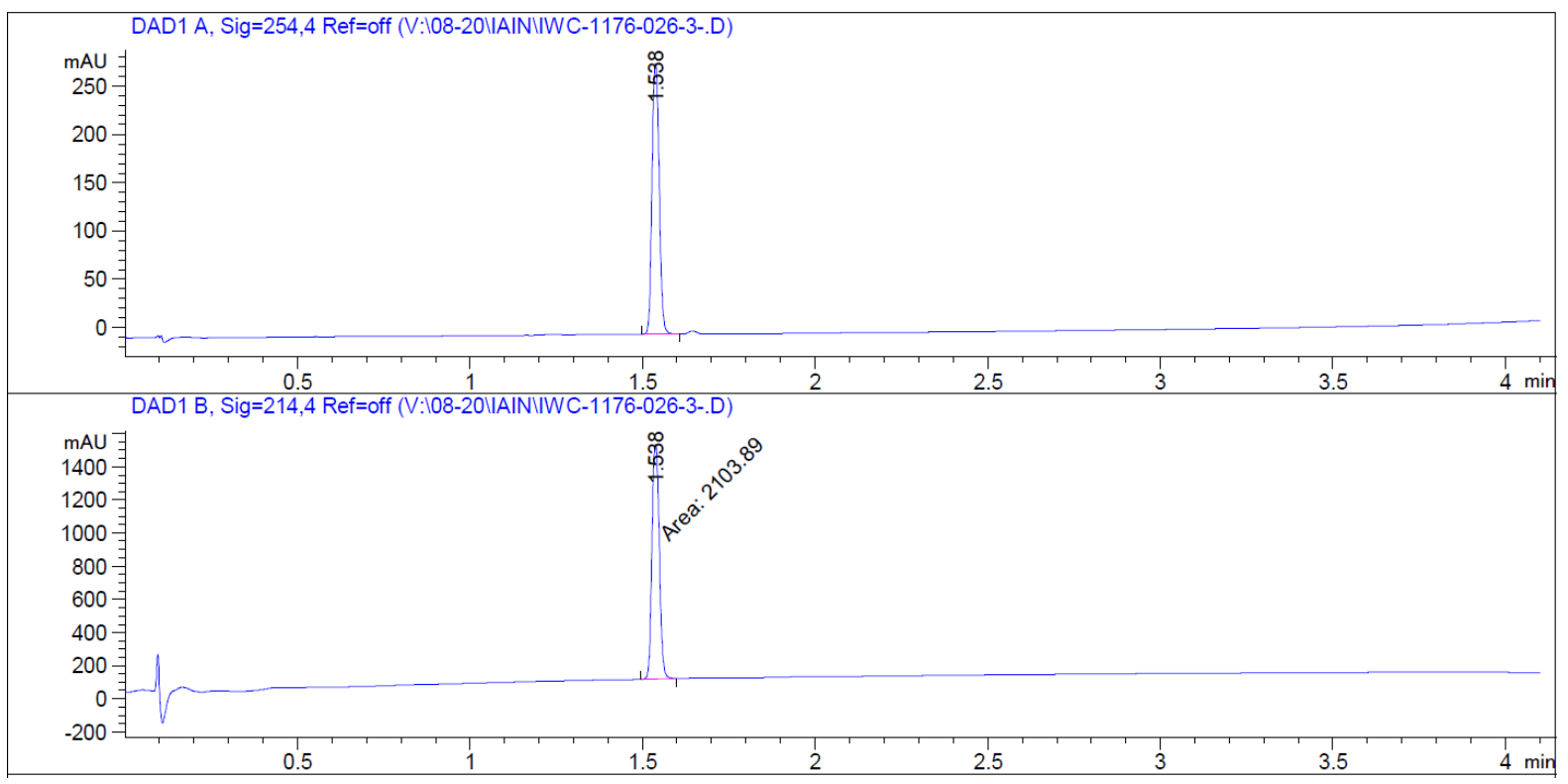


Ethyl $N$-benzoyl-P-phenylphosphonamidate (3f)

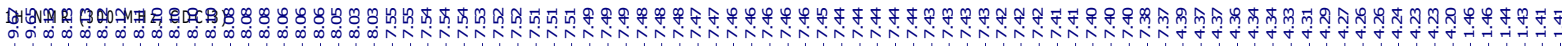
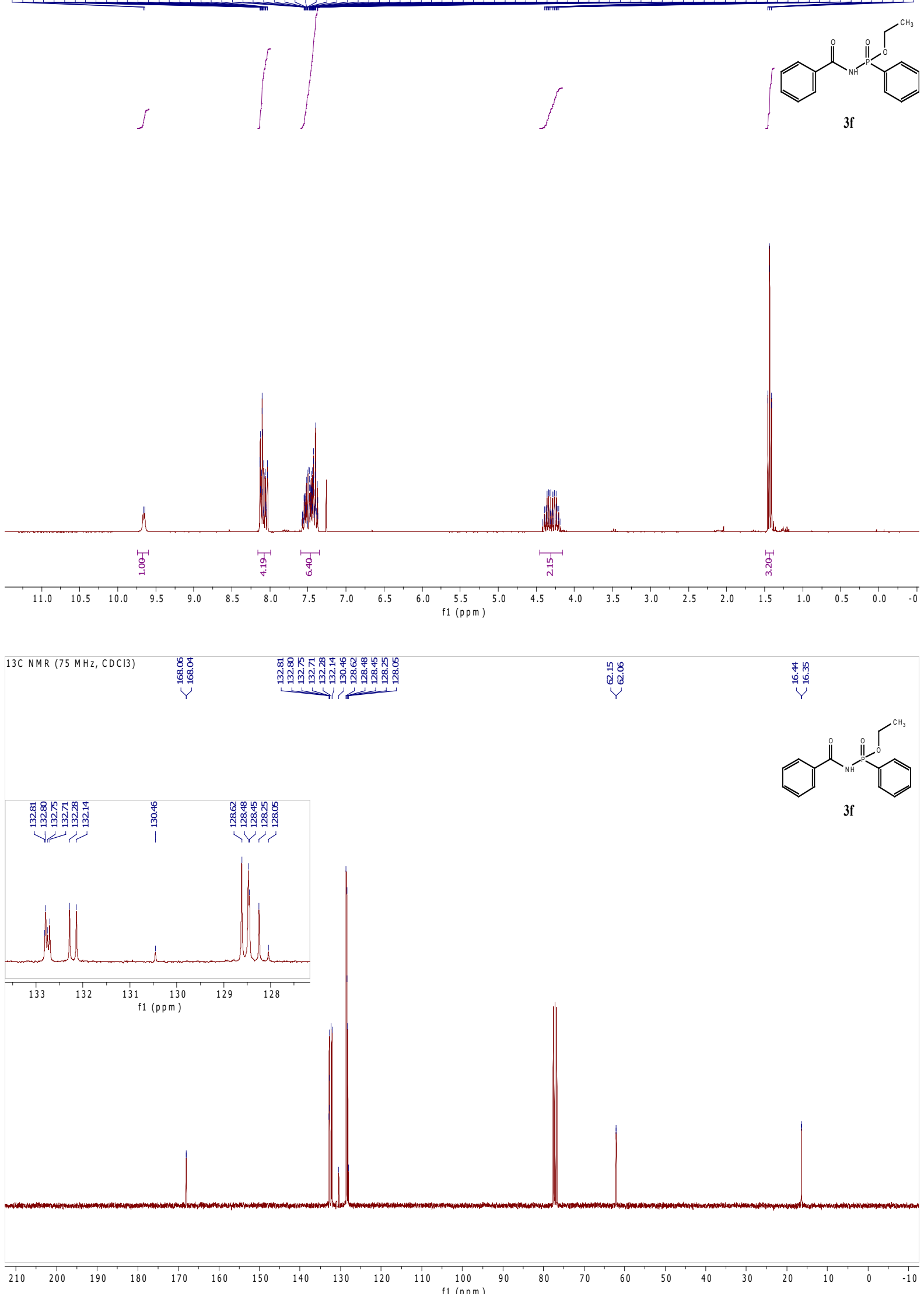


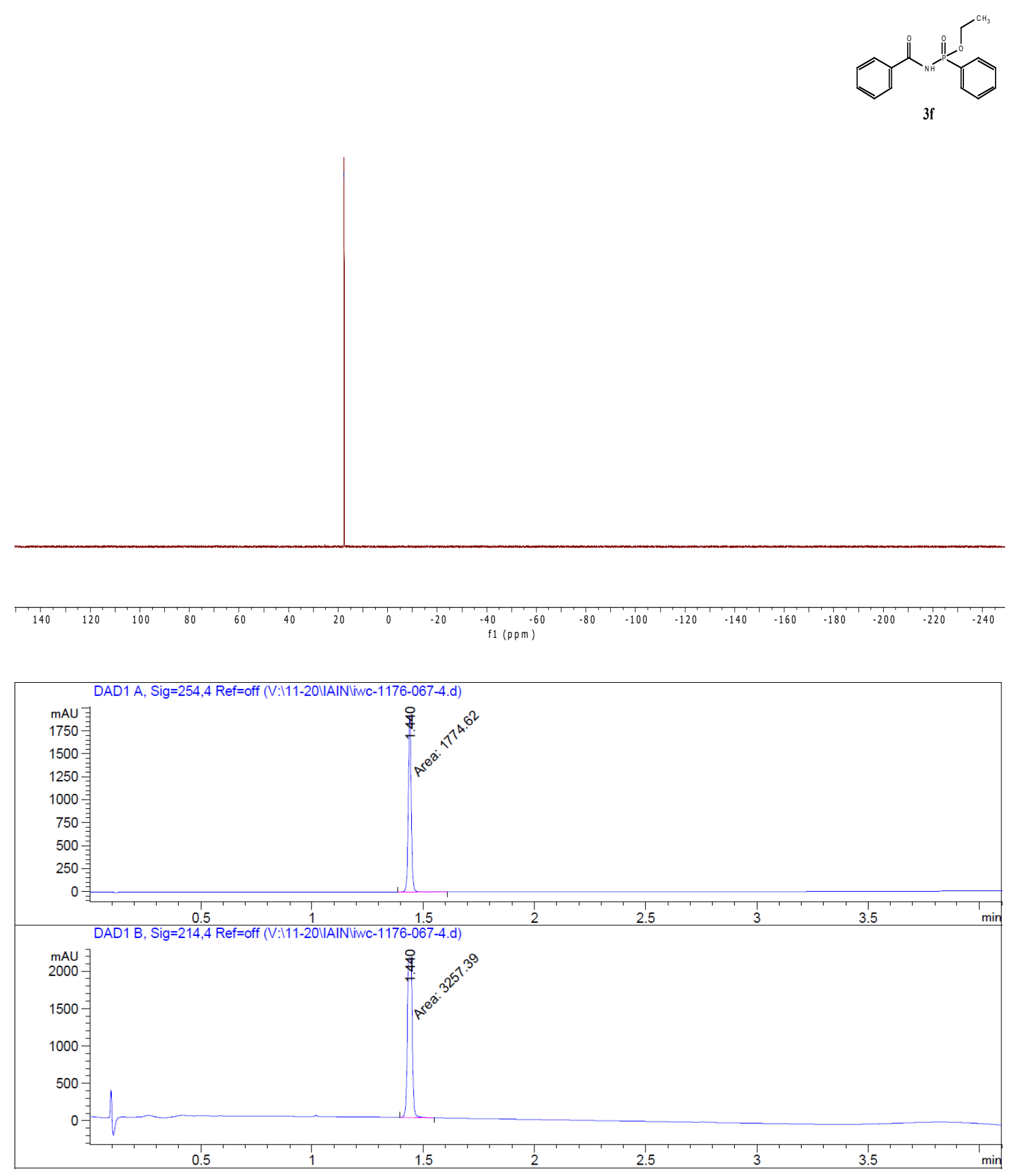


Dibenzyl diphenyl imidodiphosphate (5a)
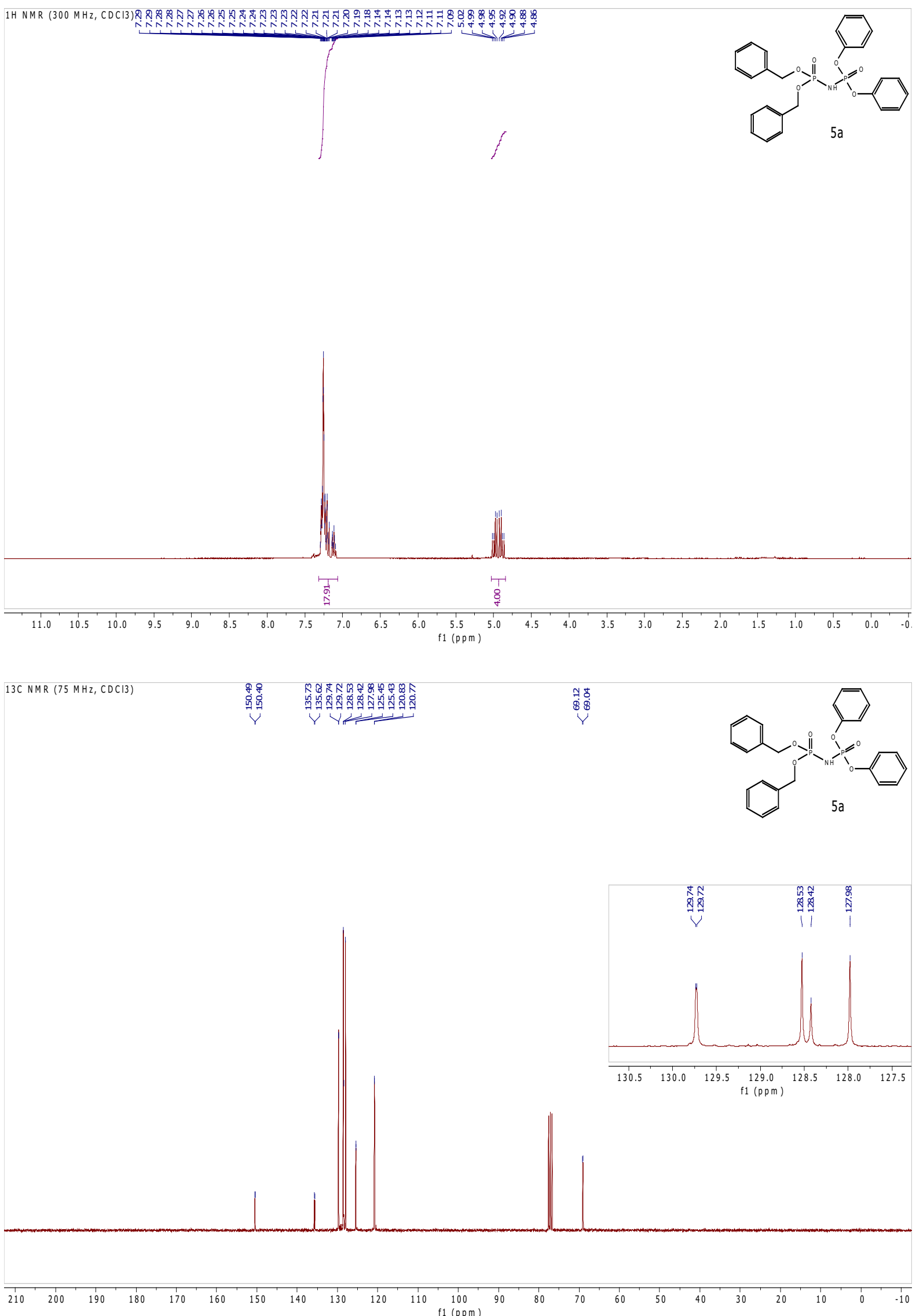


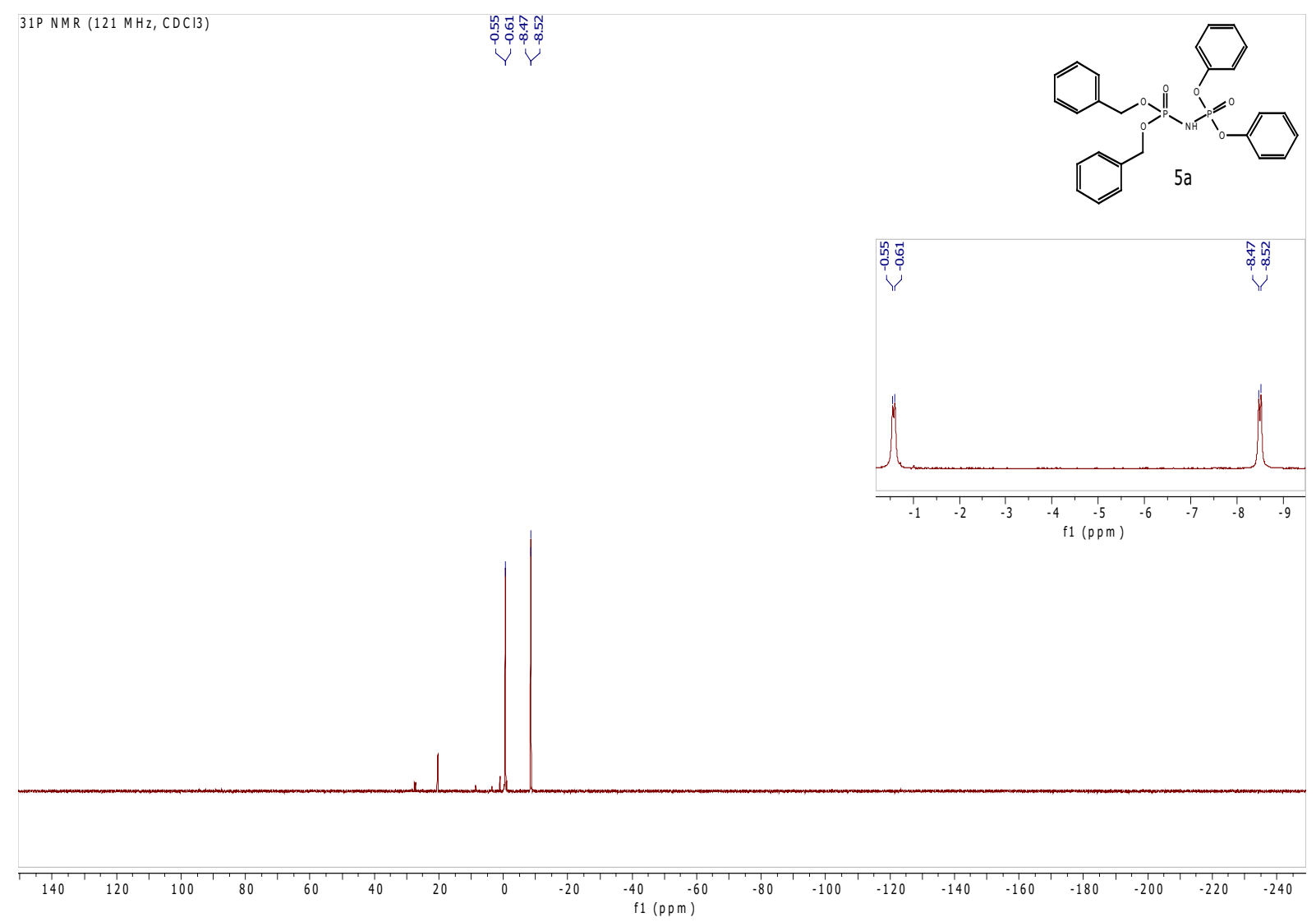

DAD1 A, Sig=254,4 Ref=off (V:I11-20VIAINliwc-1176-048-4.d)
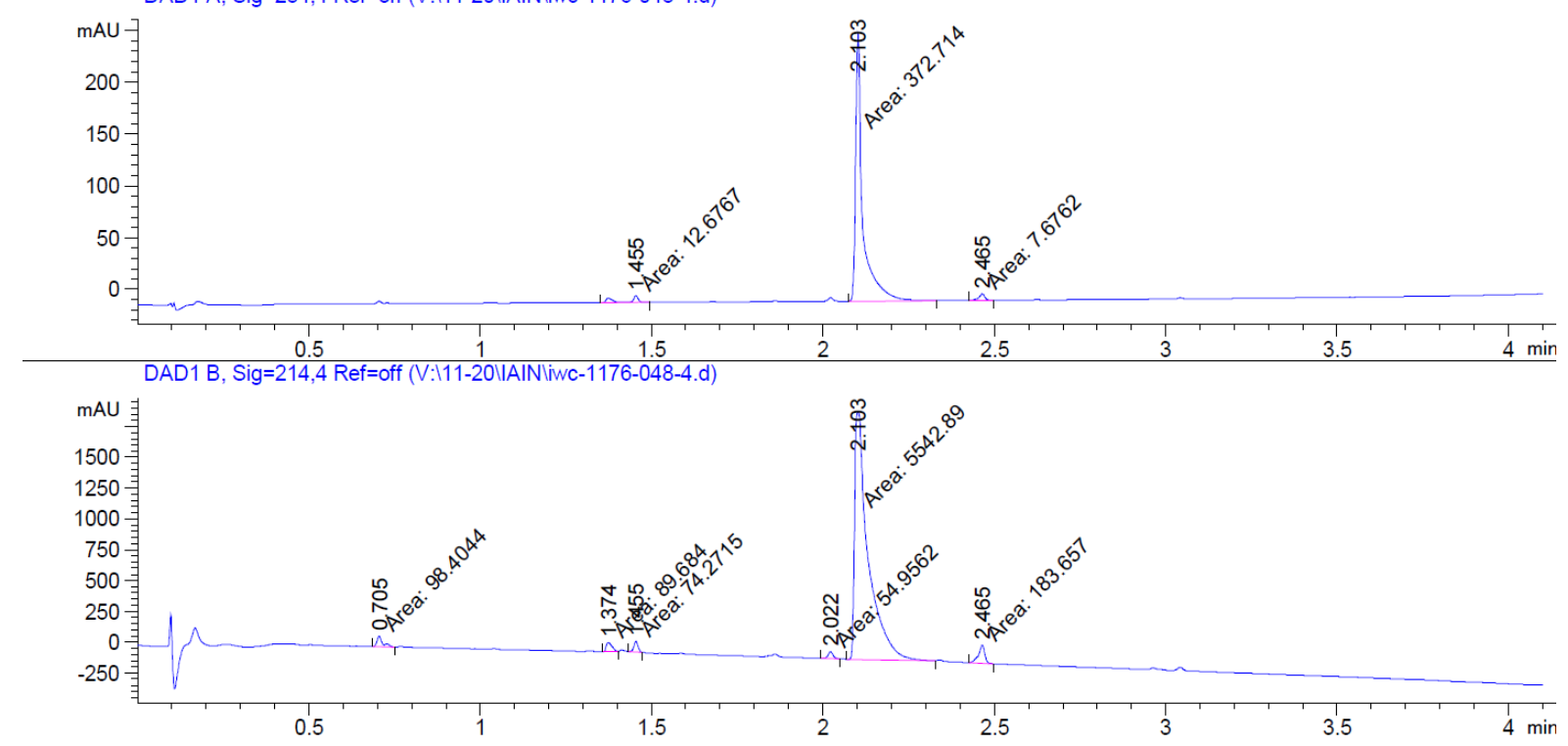
Tetraphenyl imidodiphosphate (5b)
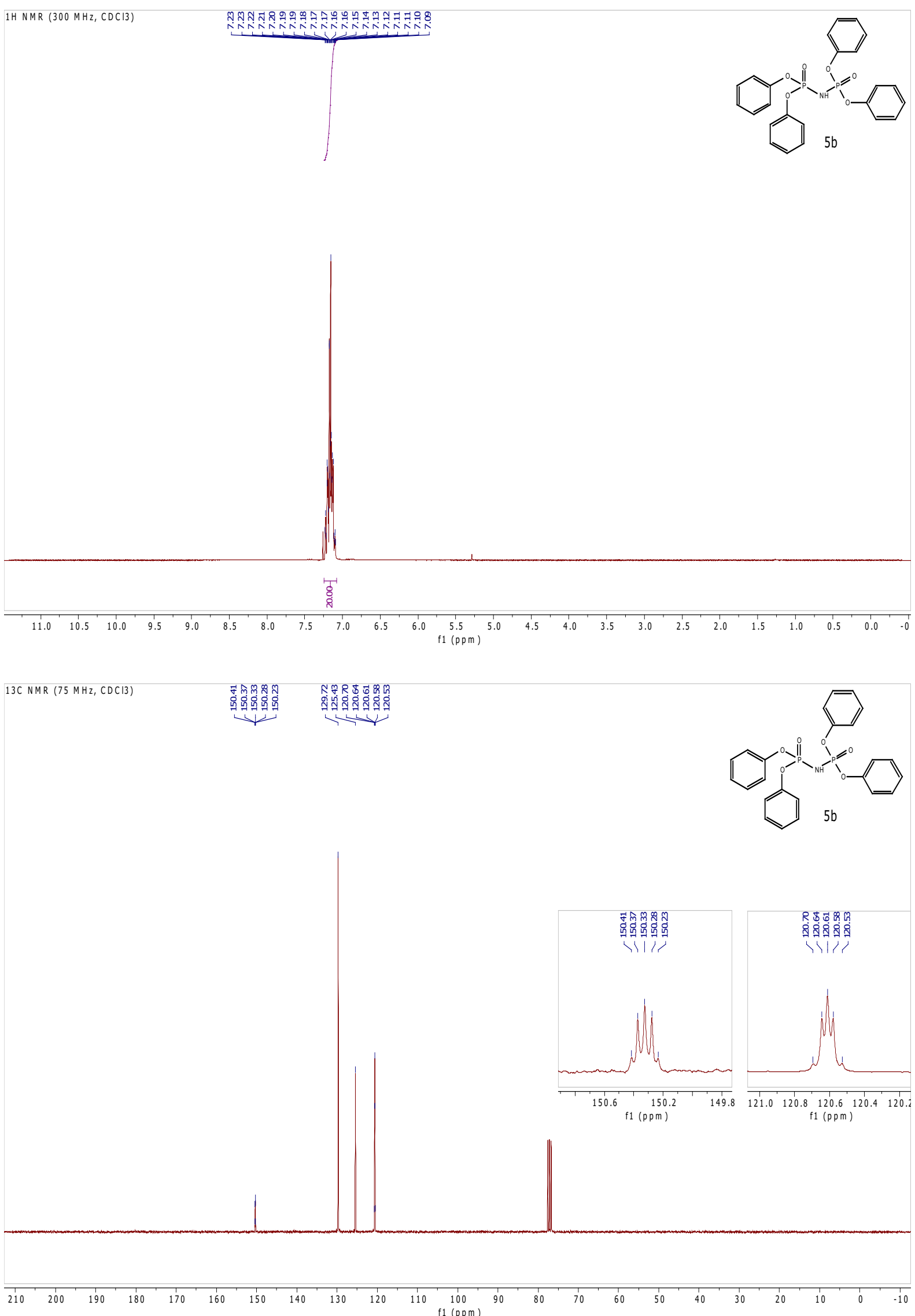

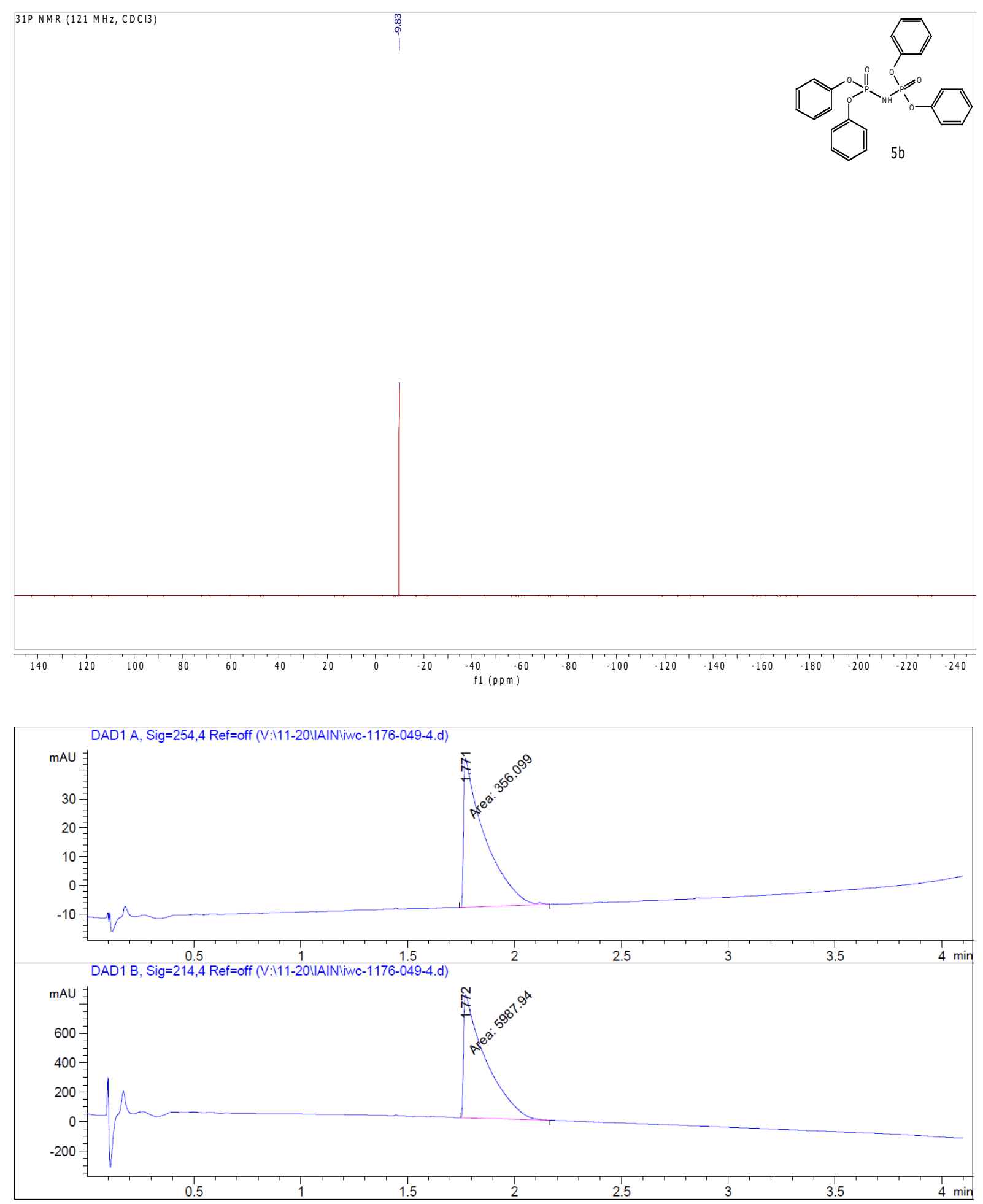
Di-tert-butyl diphenyl imidodiphosphate (5c)

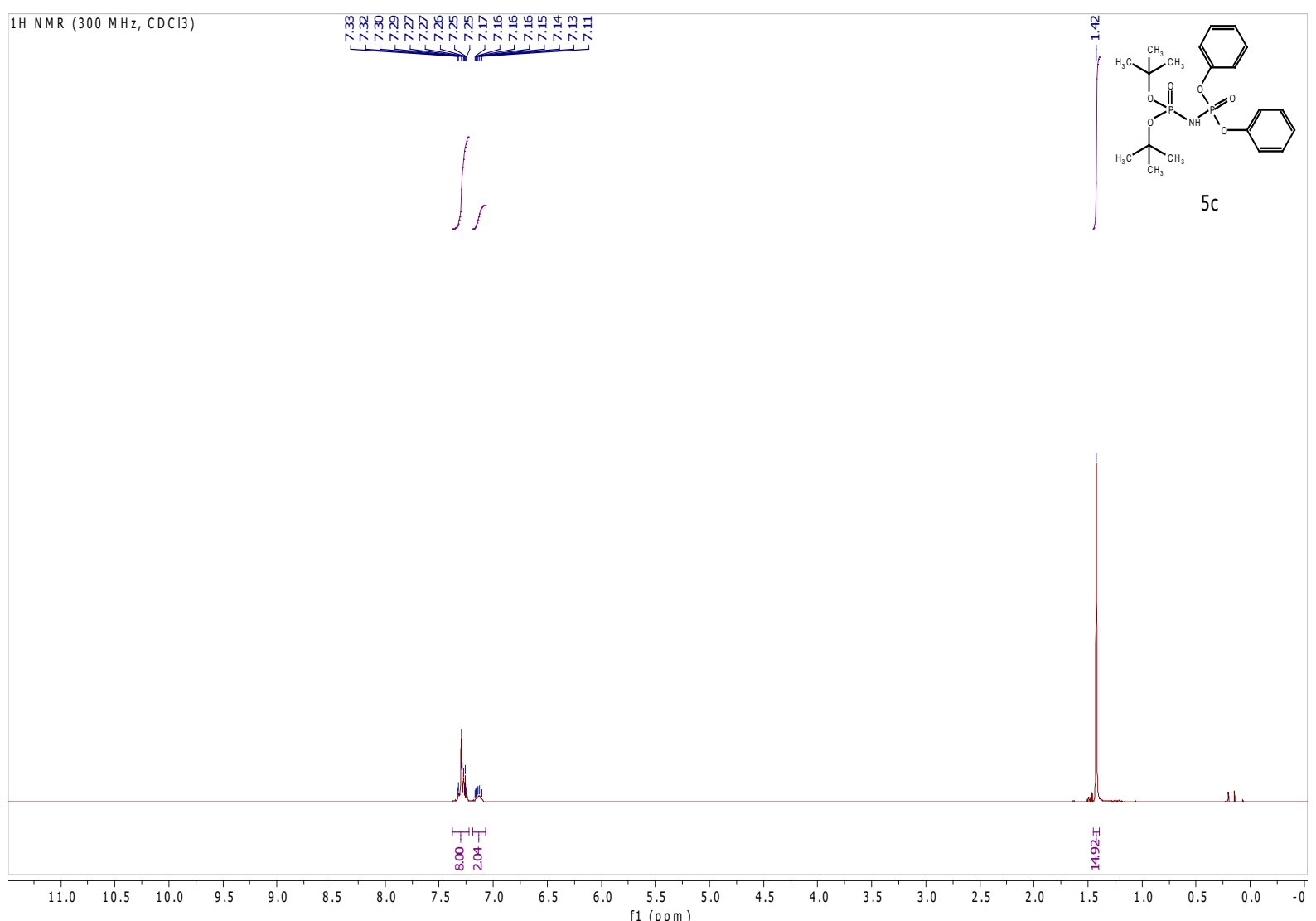




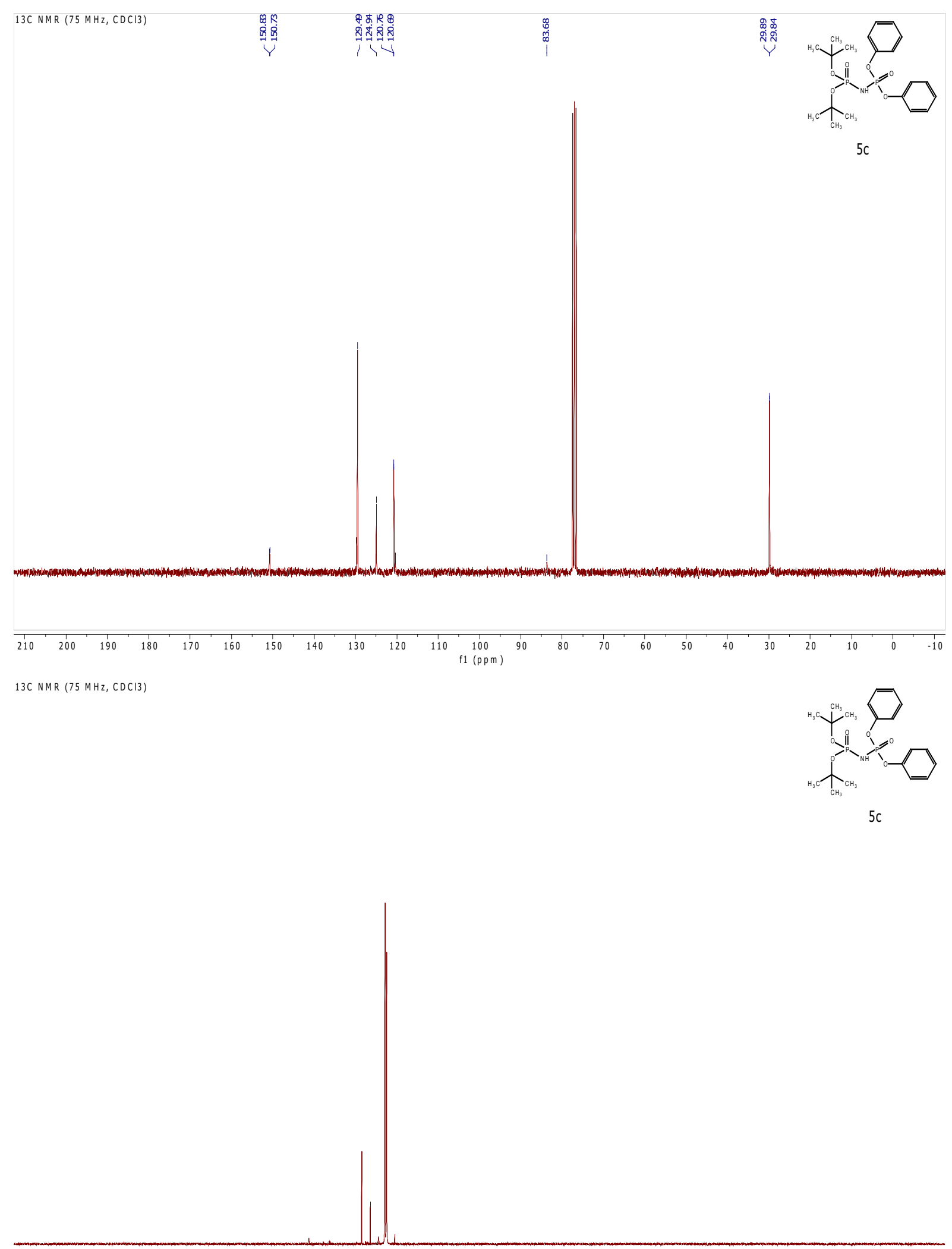

\begin{tabular}{rlllllllllllllllllllllllll}
\hline 140 & 120 & 100 & 80 & 60 & 40 & 20 & 0 & -20 & -40 & -60 & -80 & -100 & -120 & -140 & -160 & -180 & -200 & -220 & -240
\end{tabular} 
DAD1 A, Sig=254,4 Ref=off (V:111-20VIAINliwc-1176-053-4.d)

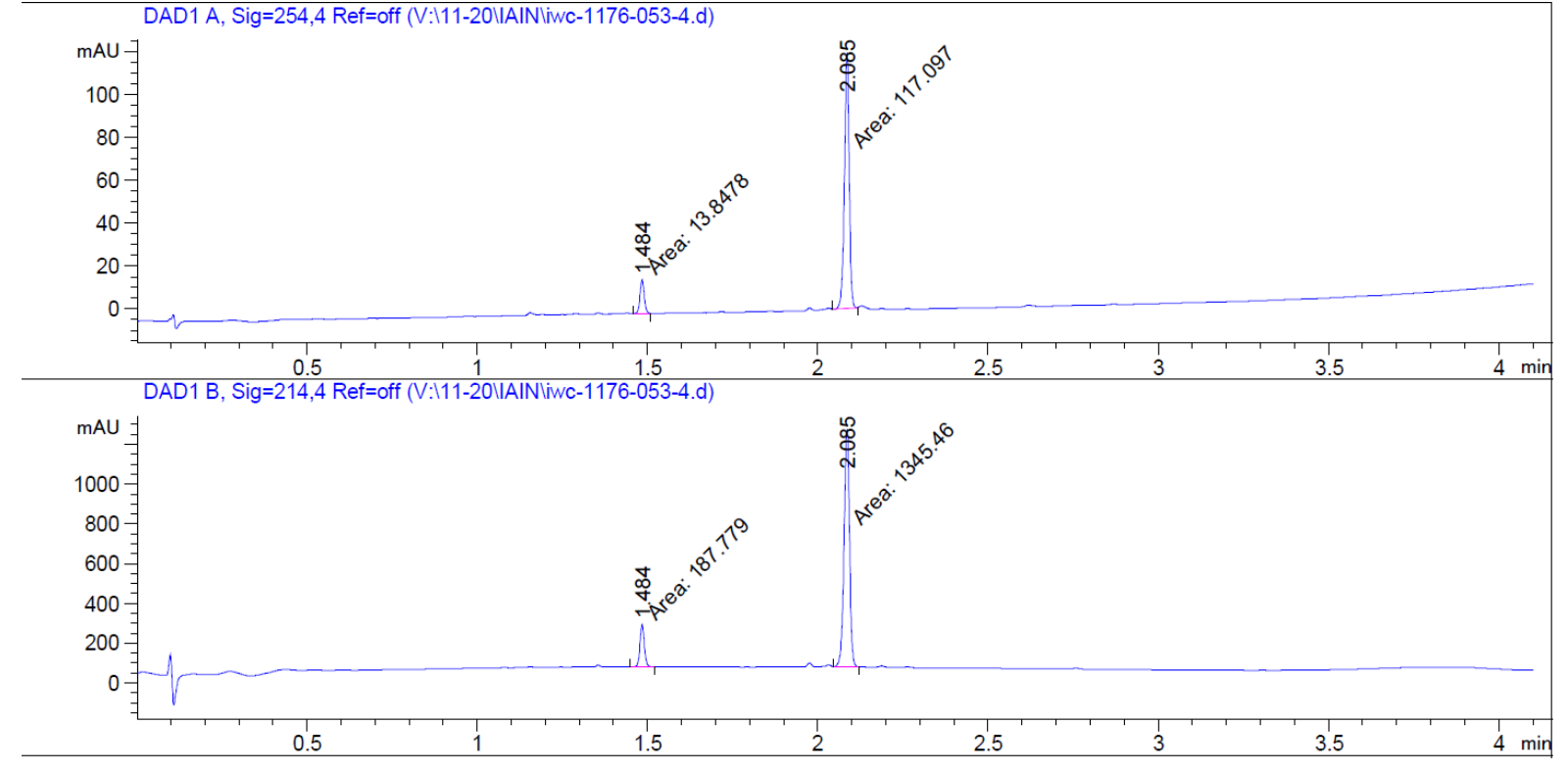


Dimethyl diphenyl imidodiphosphate (5d)
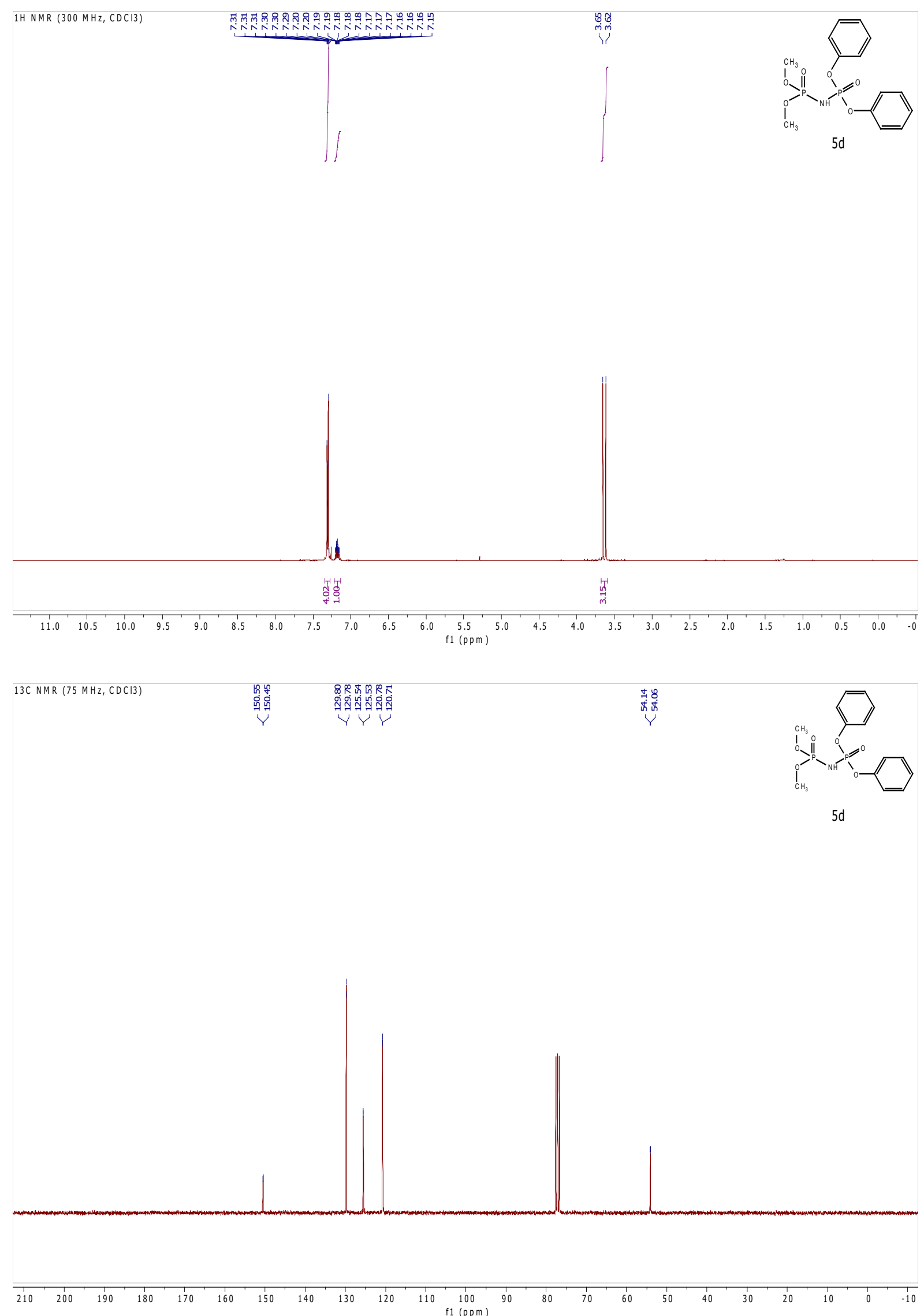

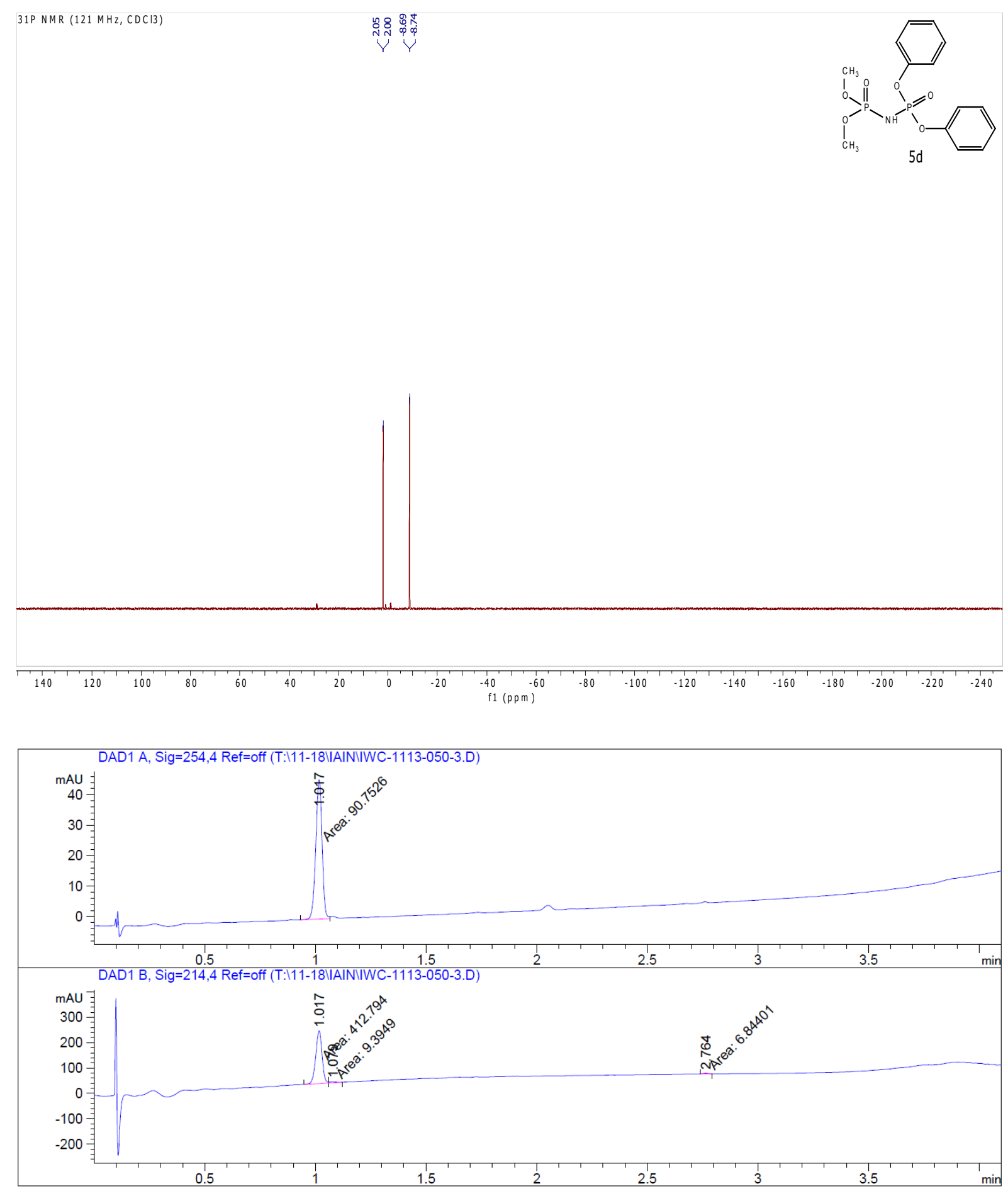


\section{Diphenyl diphenylphosphorylphosphoramidate (5e)}

1H NMR (300 MHz, CDCI3)
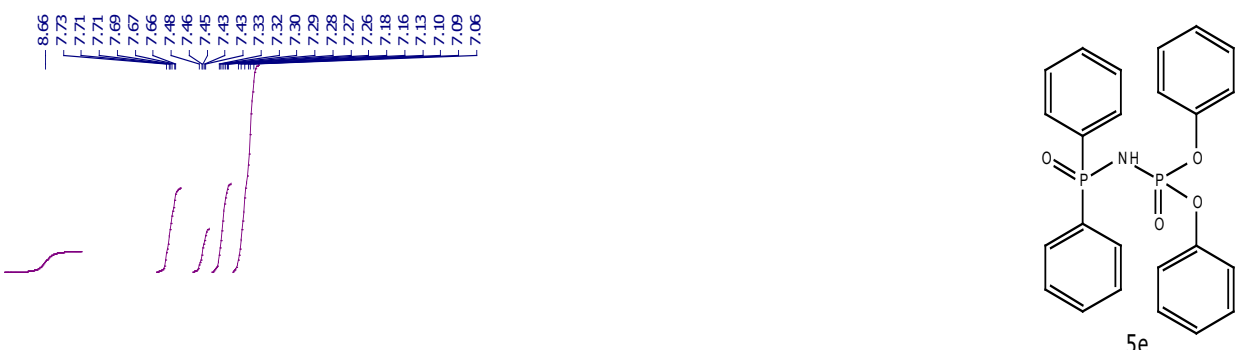

5 e

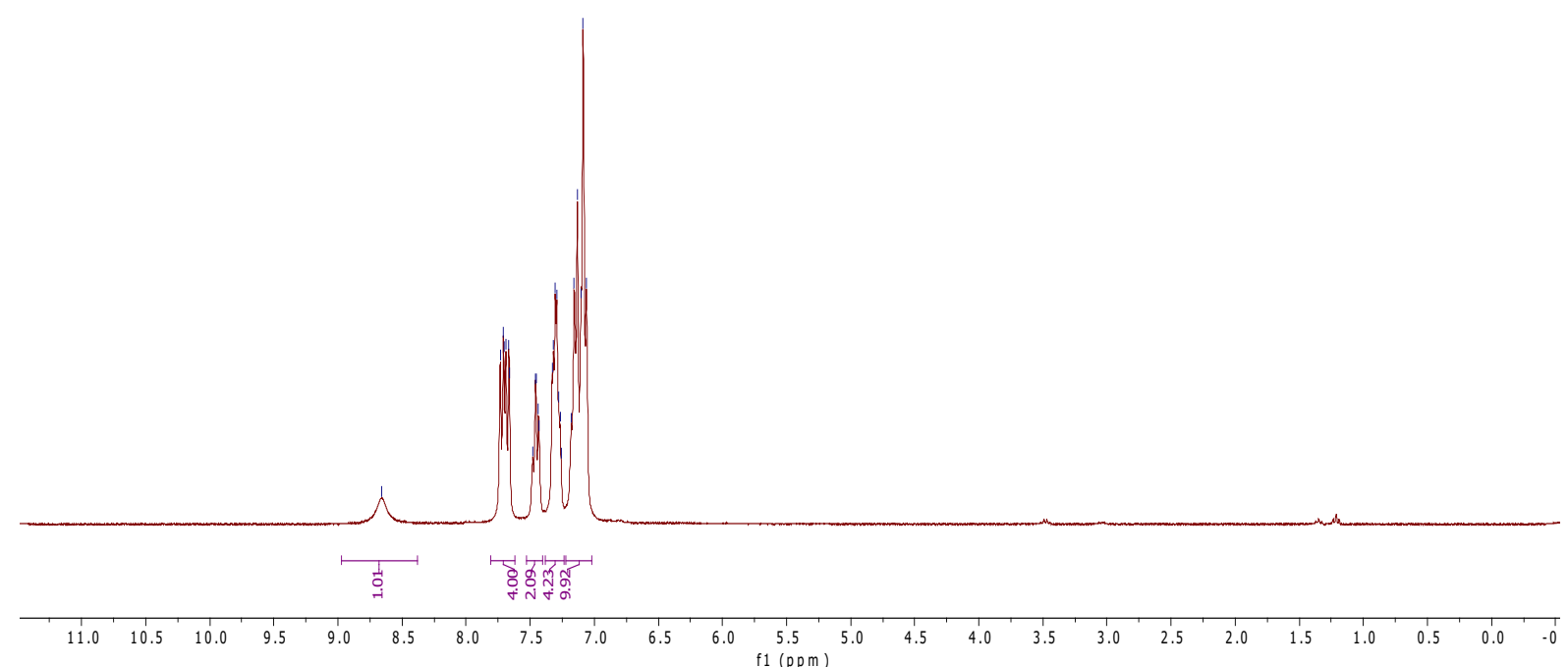

13C NMR $(75 \mathrm{MHz}, \mathrm{CDCl} 3)$

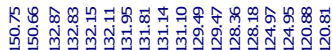
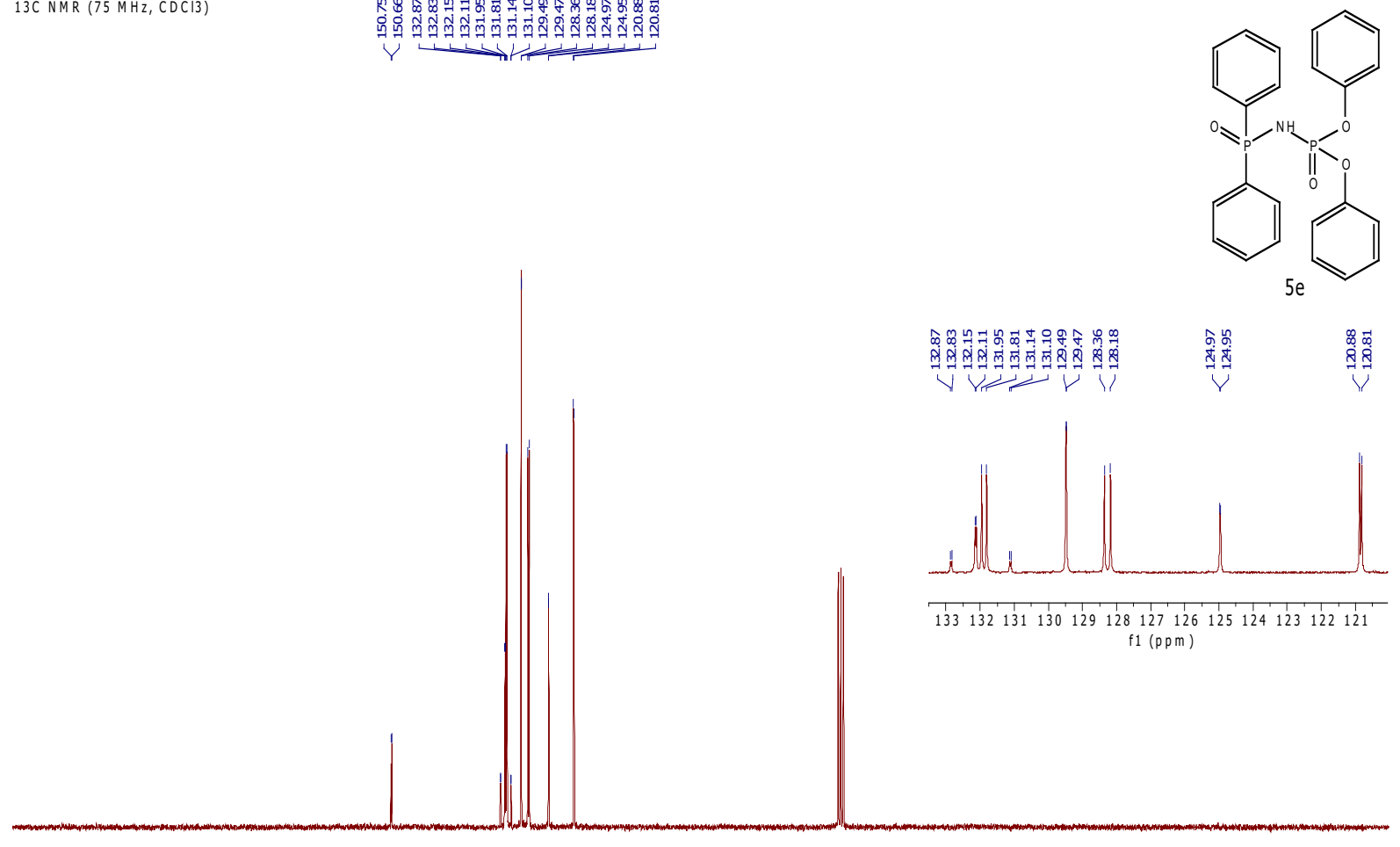

$\begin{array}{llllll}210 & 200 & 190 & 180 & 170 & 160\end{array}$

$\begin{array}{llllll}150 & 140 & 130 & 120 & 110 & 100\end{array}$ 


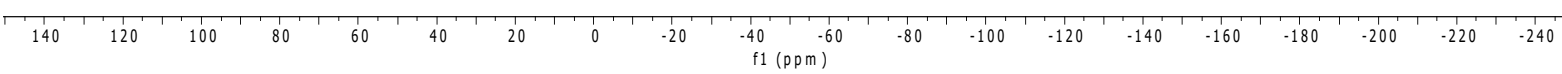

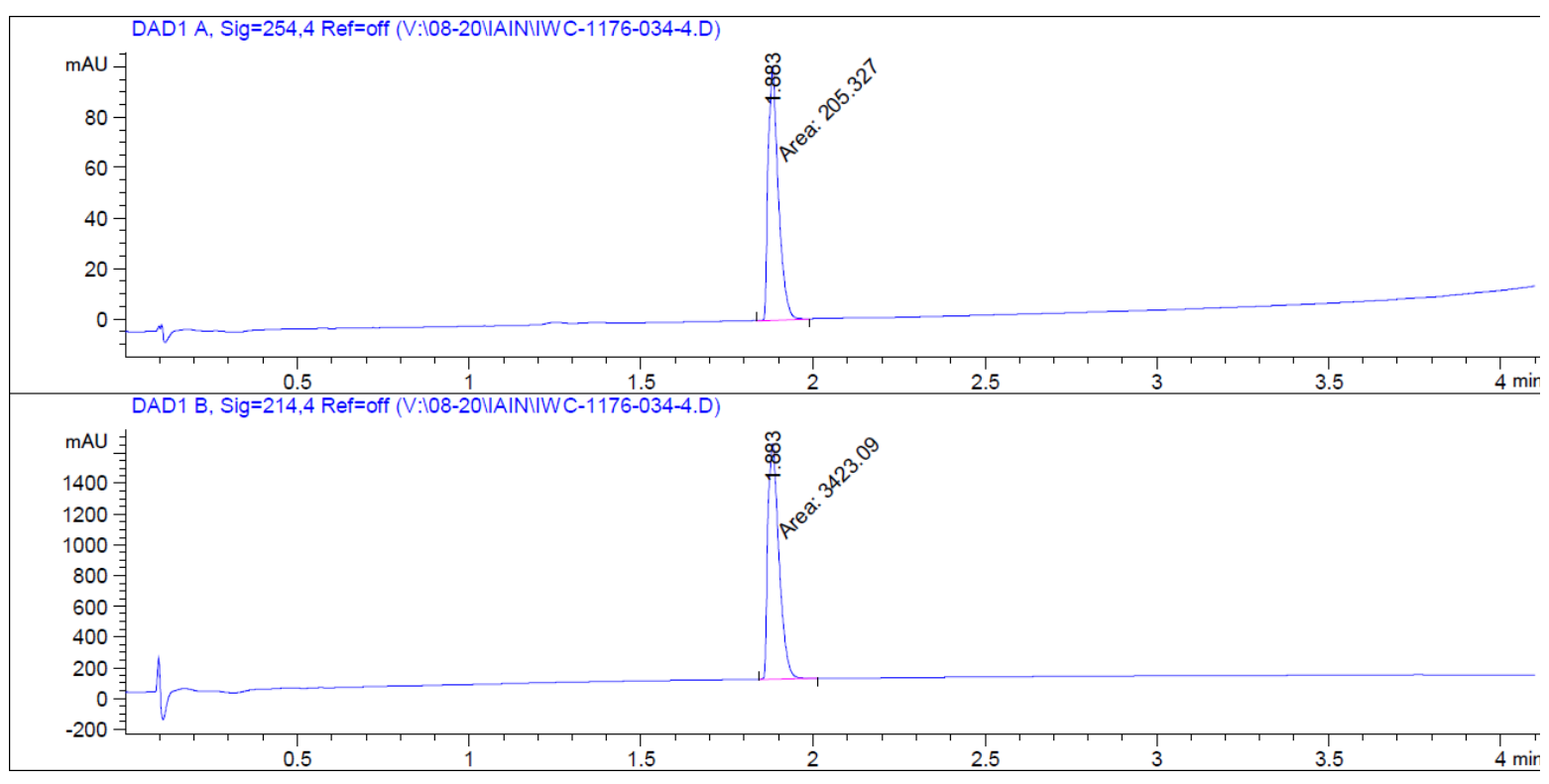


Diphenyl ethoxy(phenyl)phosphorylphosphoramidate (5f)
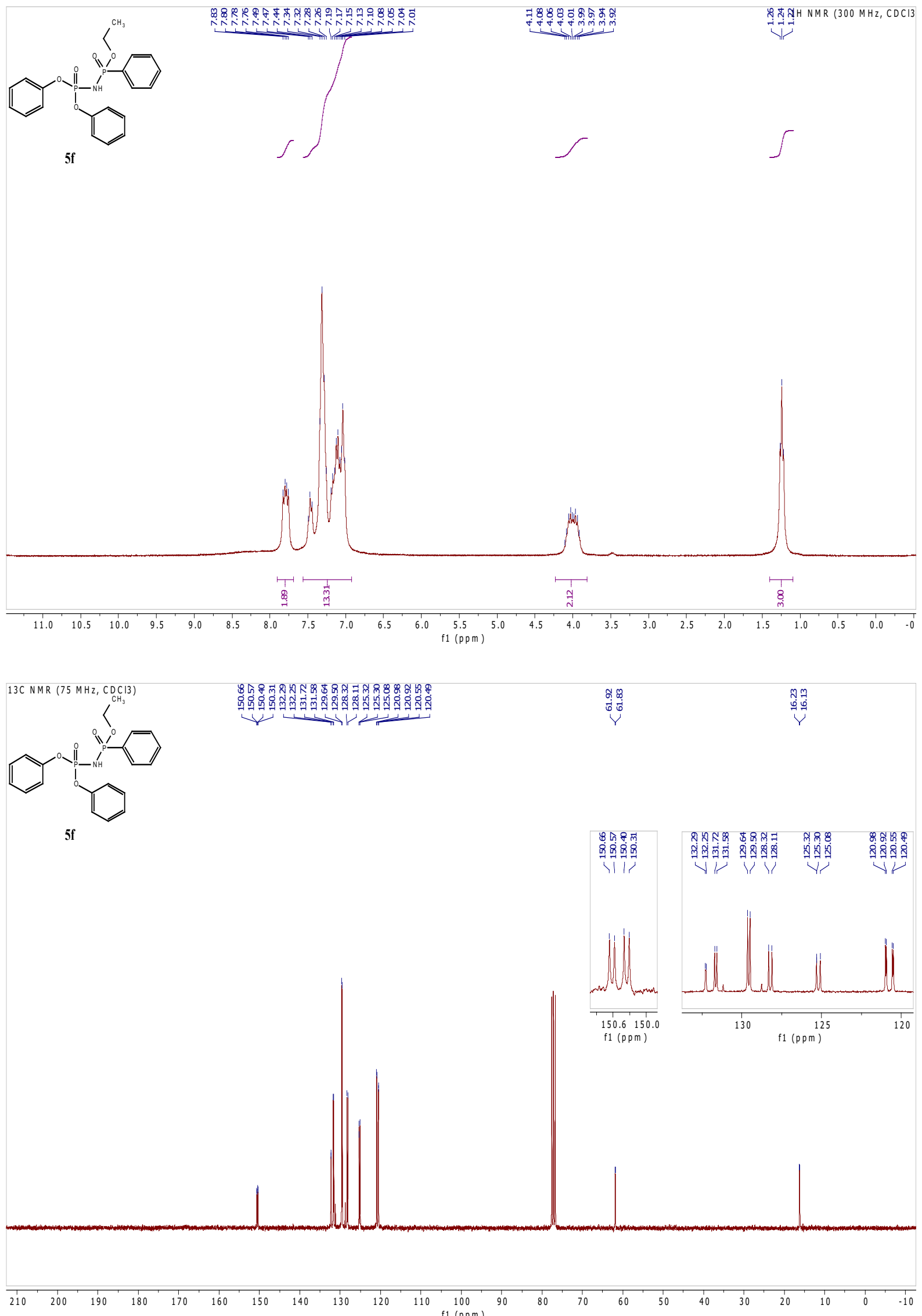
31P NMR $(121 \mathrm{MHZ}, \mathrm{CDCl} 3)$

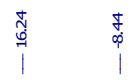
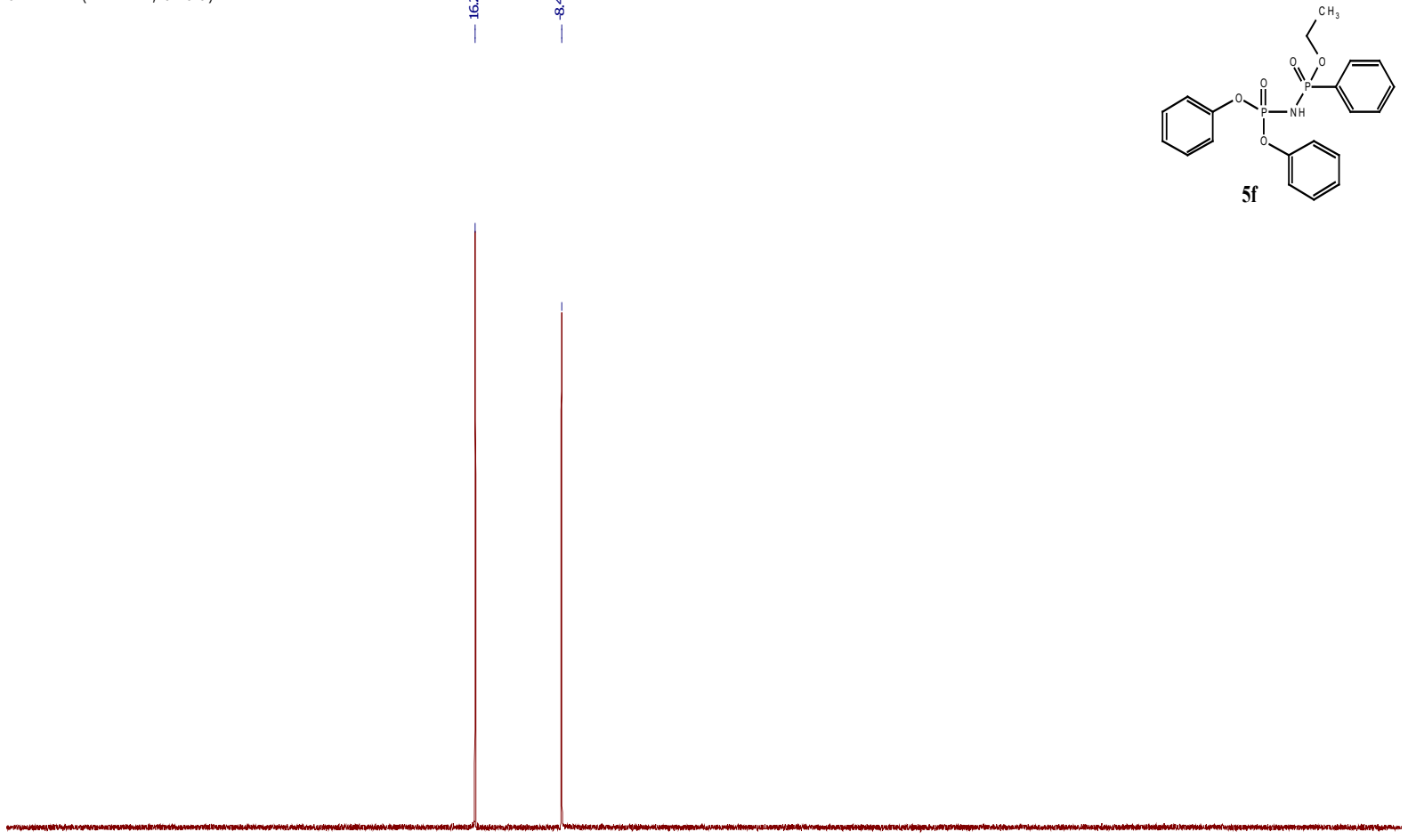

\begin{tabular}{rlllllllllllllllllllllllllllll}
\hline 140 & 120 & 100 & 80 & 60 & 40 & 20 & 0 & -20 & -40 & -60 & -80 & -100 & -120 & -140 & -160 & -180 & -200 & -220 & -240
\end{tabular}

DAD1 A, Sig=254,4 Ref=off (V:I11-20VAINliwc-1176-063-4.d)

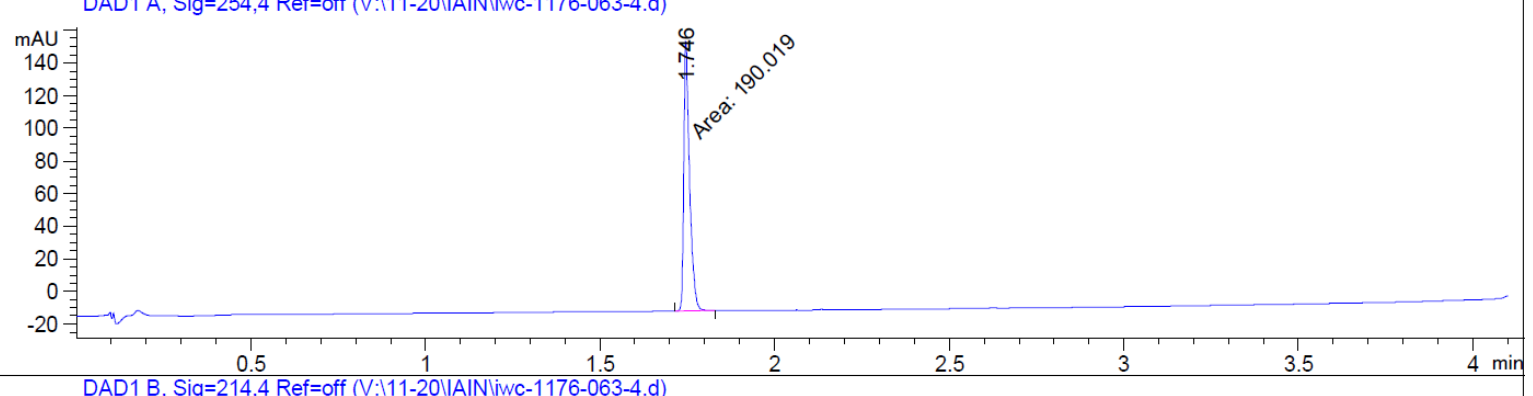

DAD1 B, Sig=214,4 Ref=off (V:I11-20VAINliwc-1176-063-4.d)

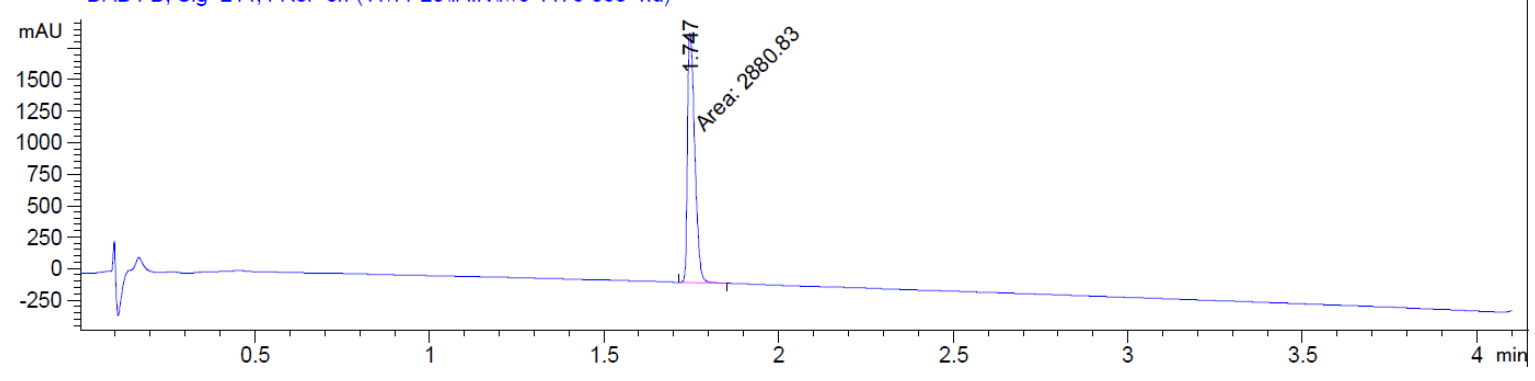


Dibenzyl thiophene-2-carbonylphosphoramidate (3g)

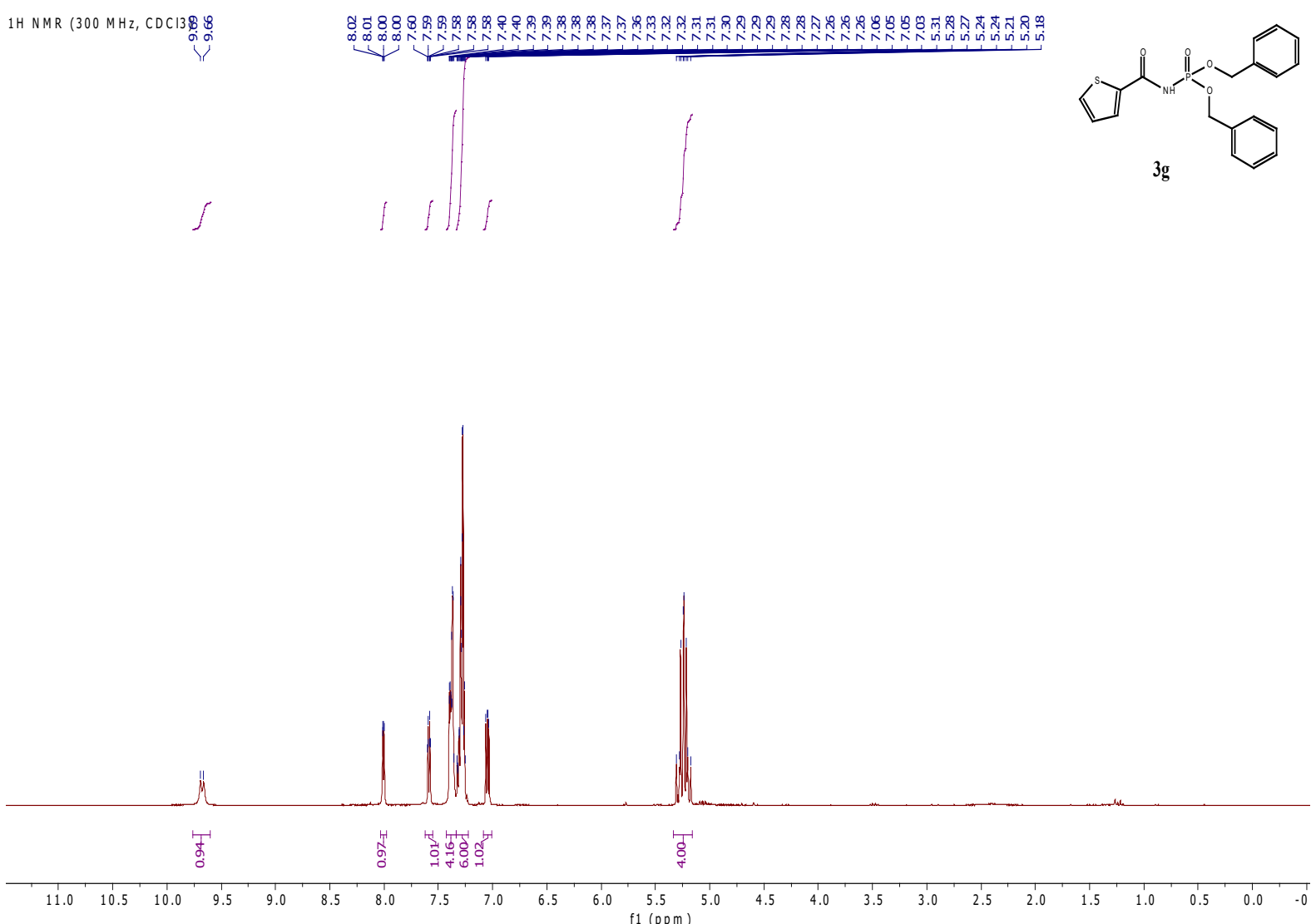

\begin{tabular}{|c|c|c|}
\hline $13 \mathrm{CNMR}(75 \mathrm{MHz}, \mathrm{CDCl3})$ & 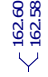 & 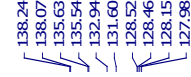 \\
\hline
\end{tabular}

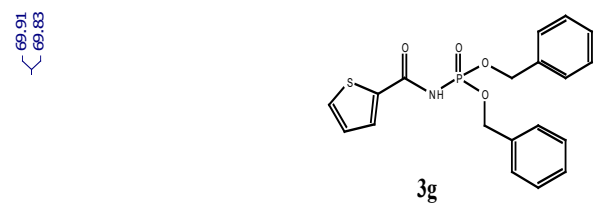

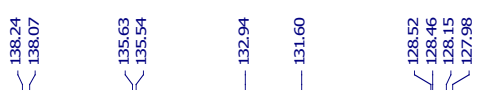

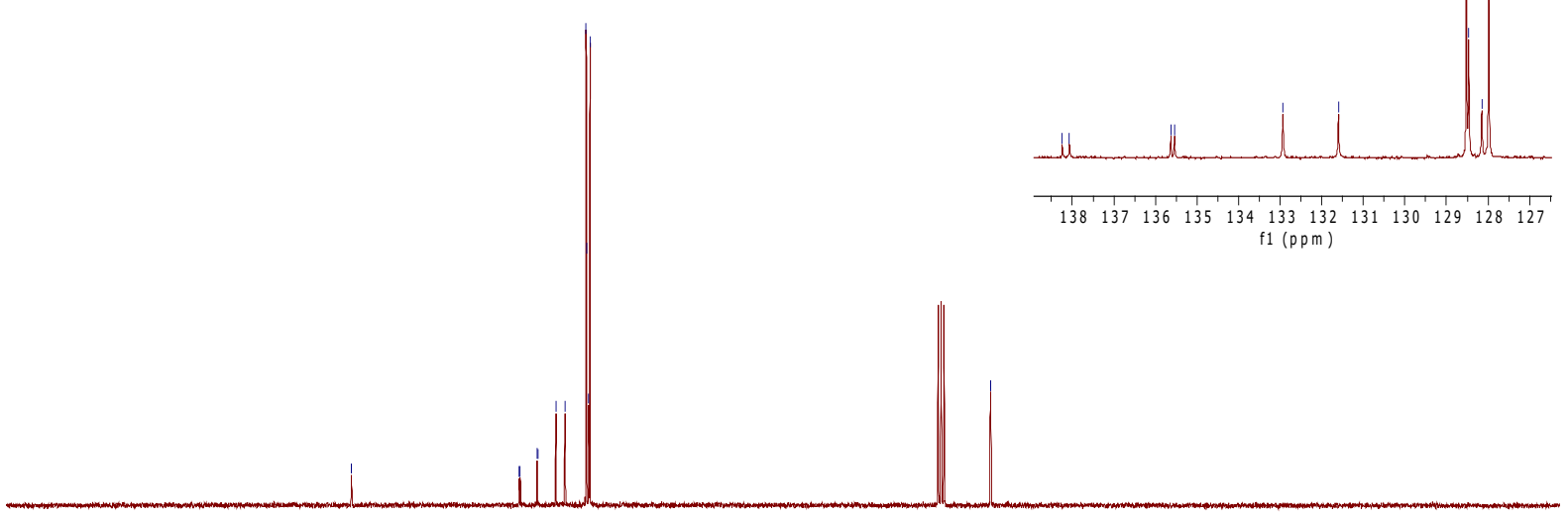

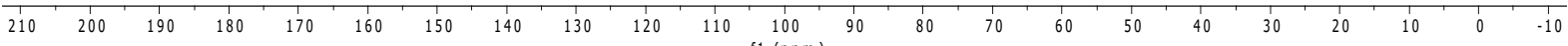



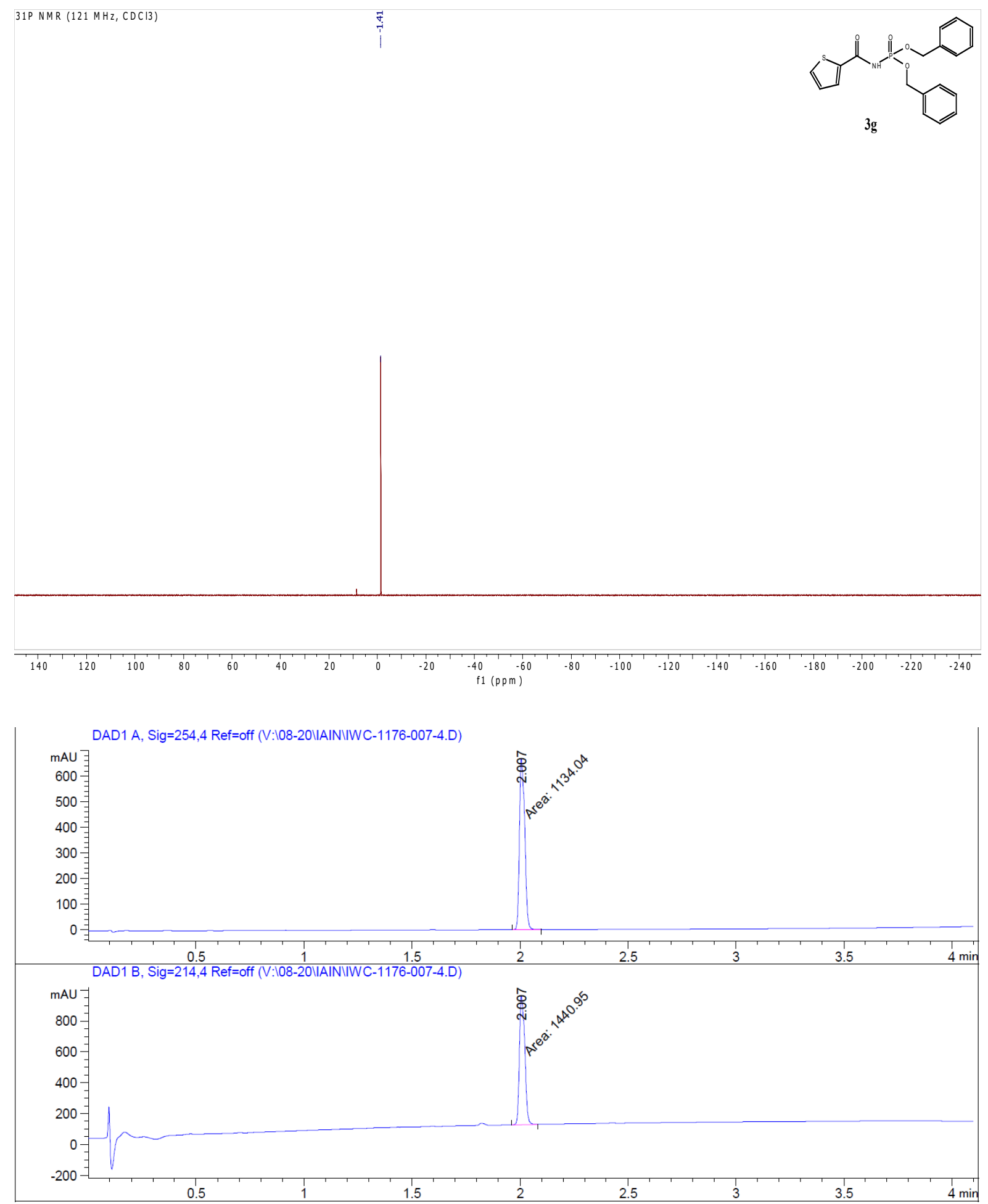
Dibenzyl furan-2-carbonylphosphoramidate (3h)

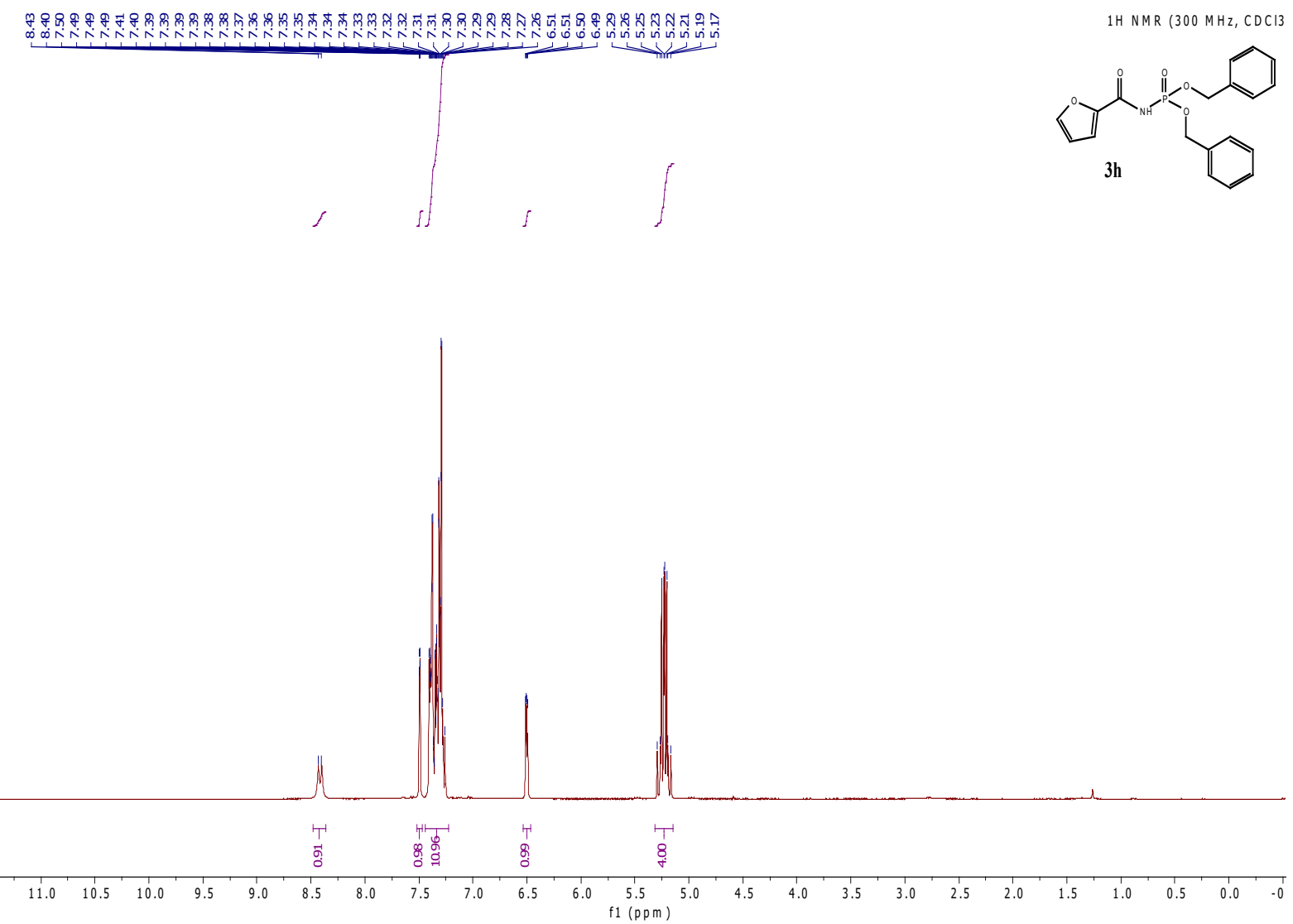

$13 \mathrm{CNMR}(75 \mathrm{MHz}, \mathrm{CDCl}) \quad$ S

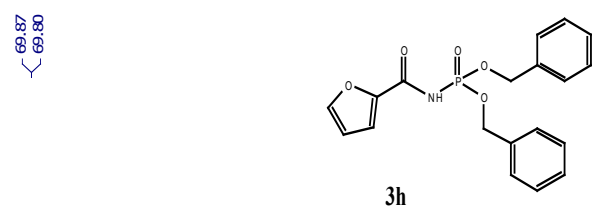

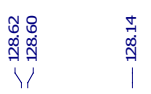

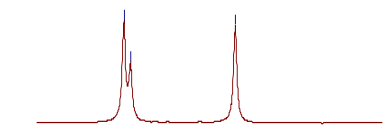

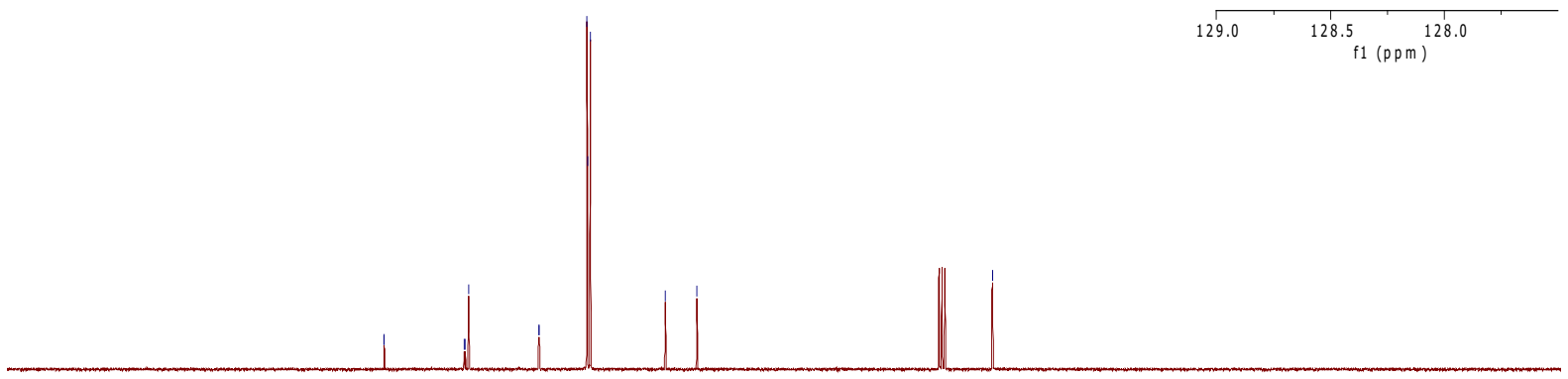

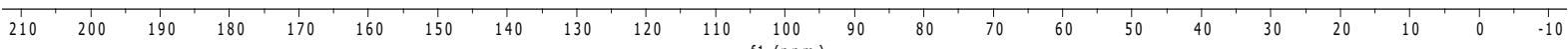



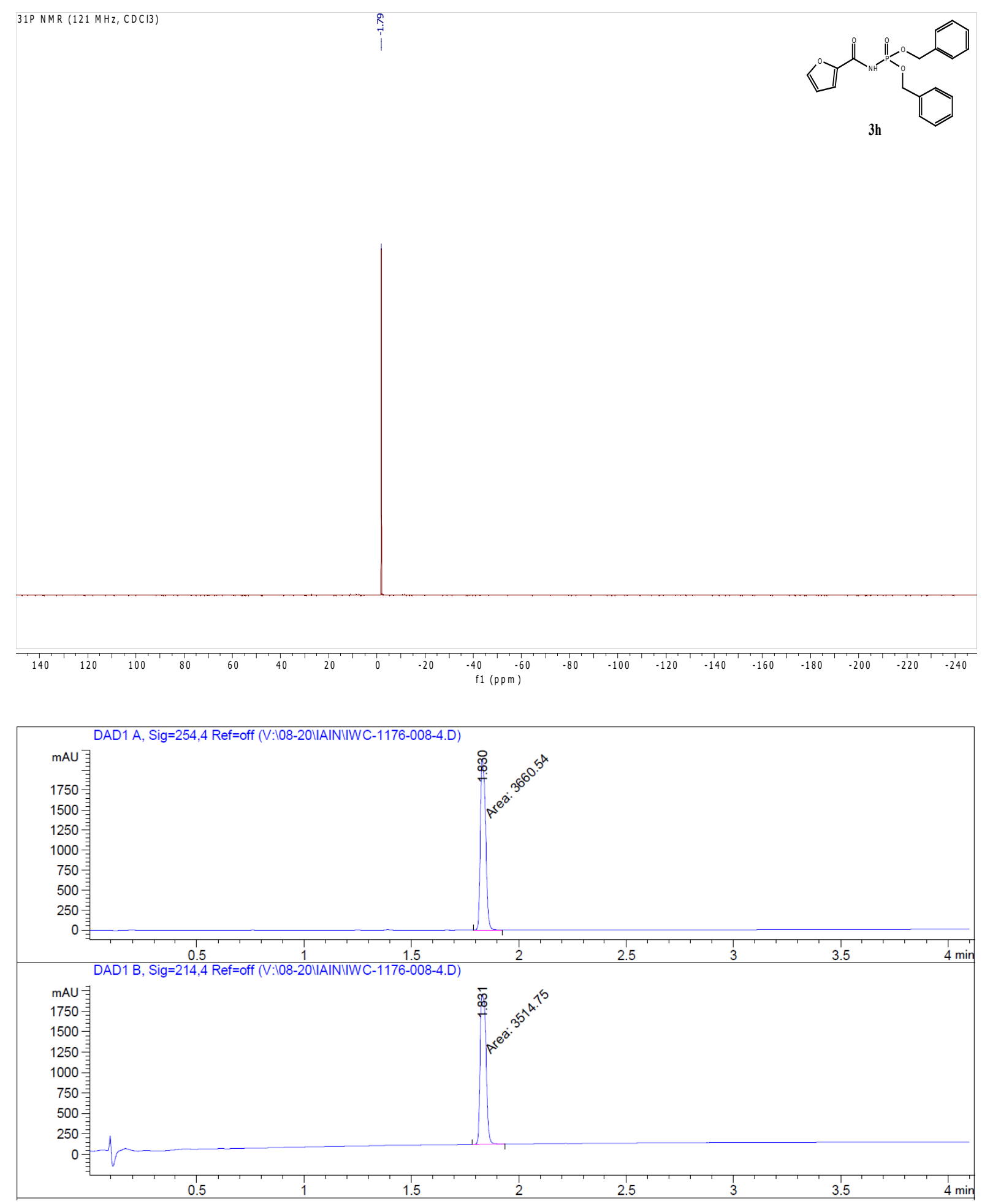
(E)-Dibenzyl cinnamoylphosphoramidate (3i)

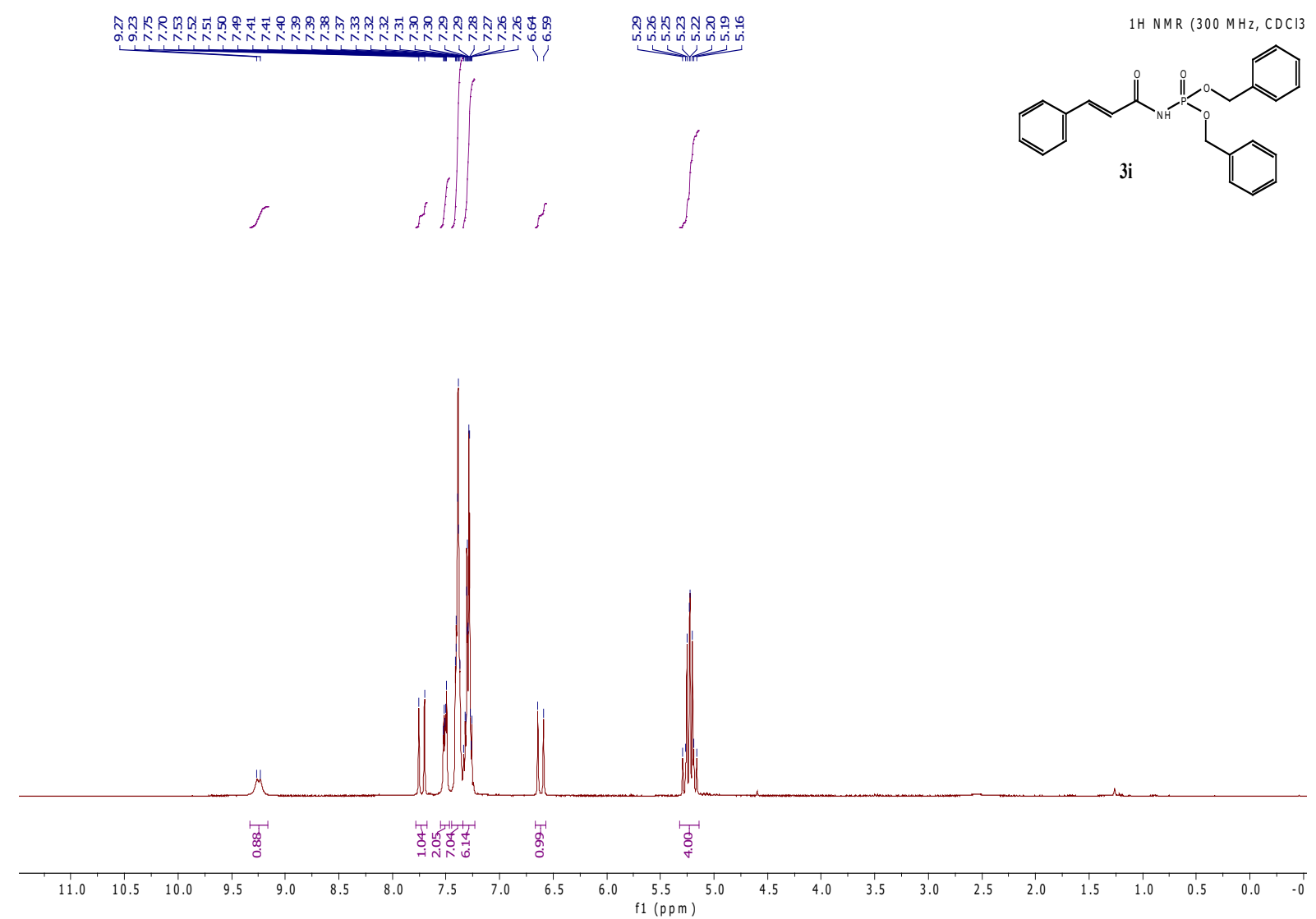

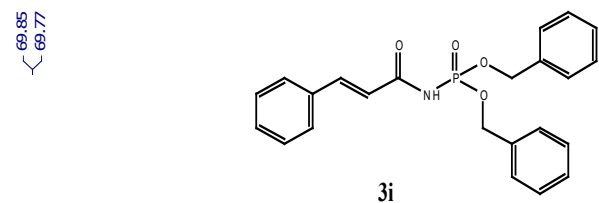

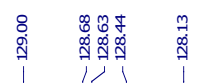

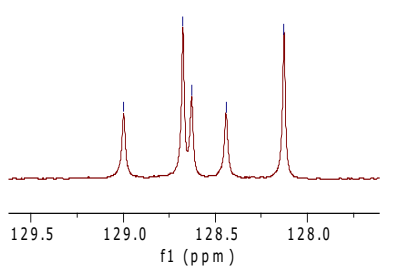

$\begin{array}{lllllllllllllllllllllll}1 & 1 \\ 210 & 200 & 190 & 180 & 170 & 160 & 150 & 140 & 130 & 120 & 110 & 100 & 90 & 80 & 70 & 60 & 50 & 40 & 30 & 20 & 10 & 0 & -10\end{array}$ 


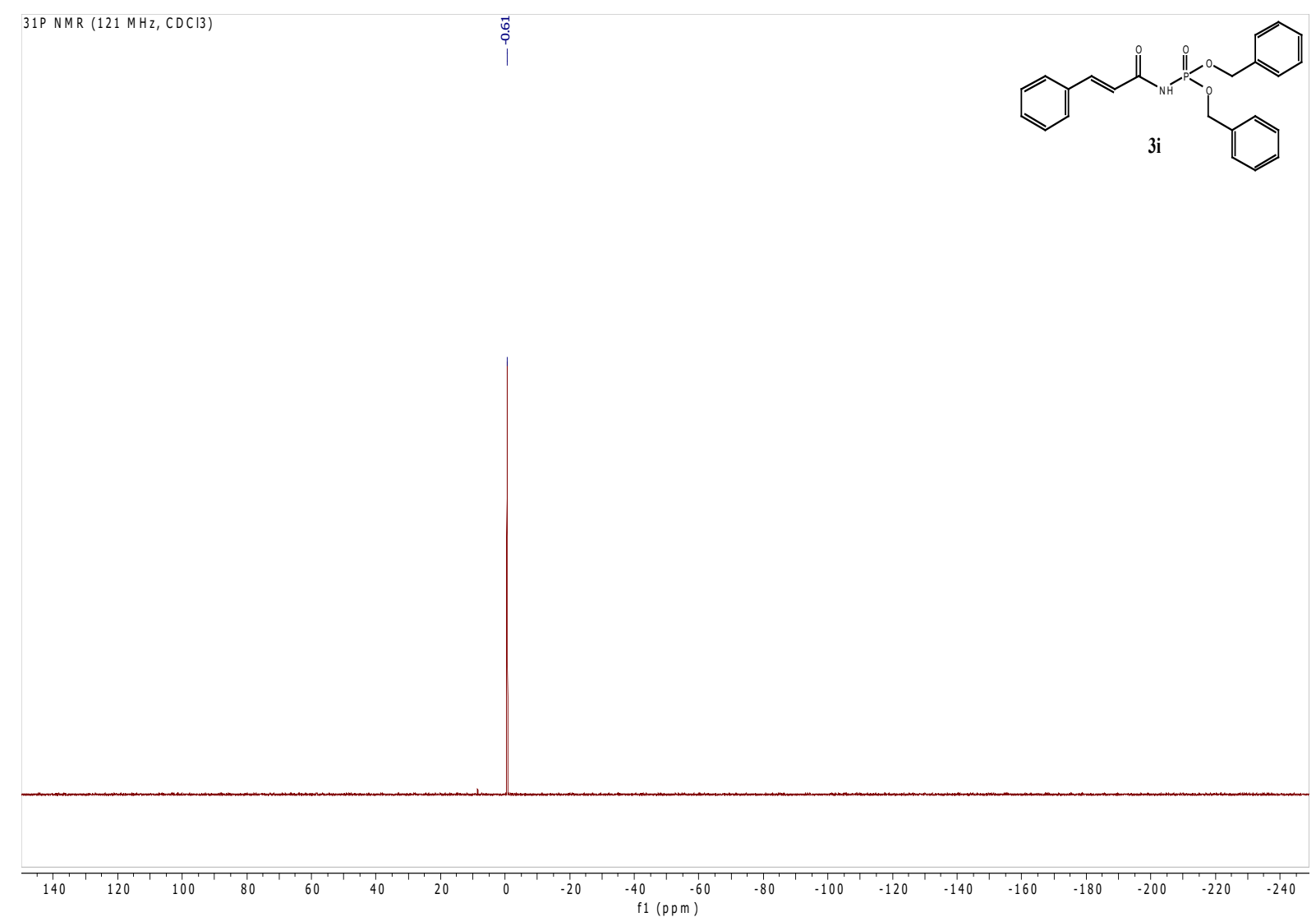

DAD1 A, Sig=254,4 Ref=off (V:108-20VIINIIWC-1176-009-4.D)

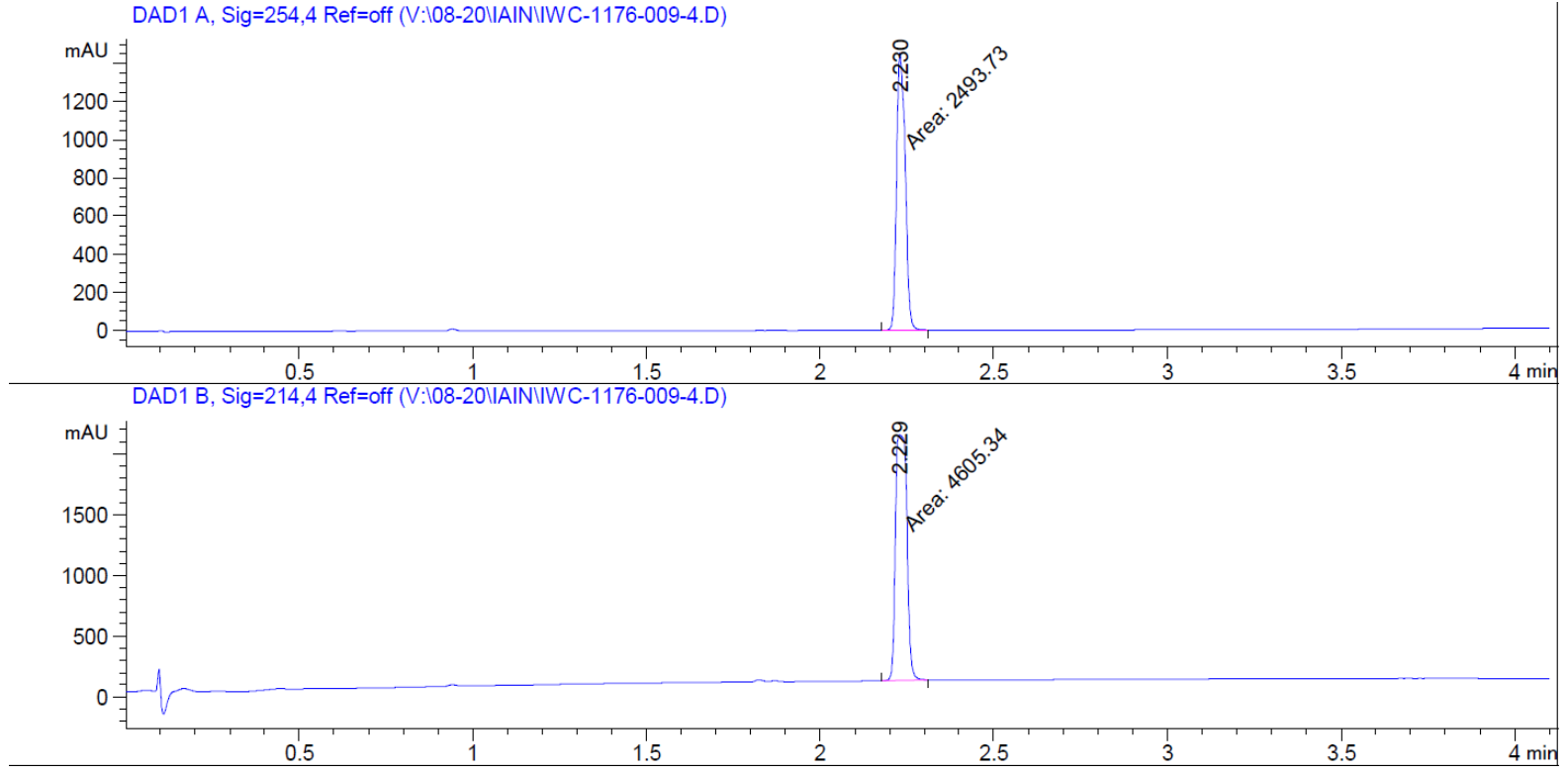


Dibenzyl picolinoylphosphoramidate (3j)

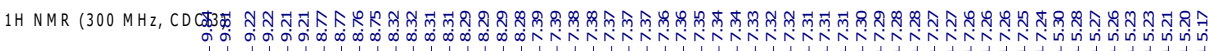
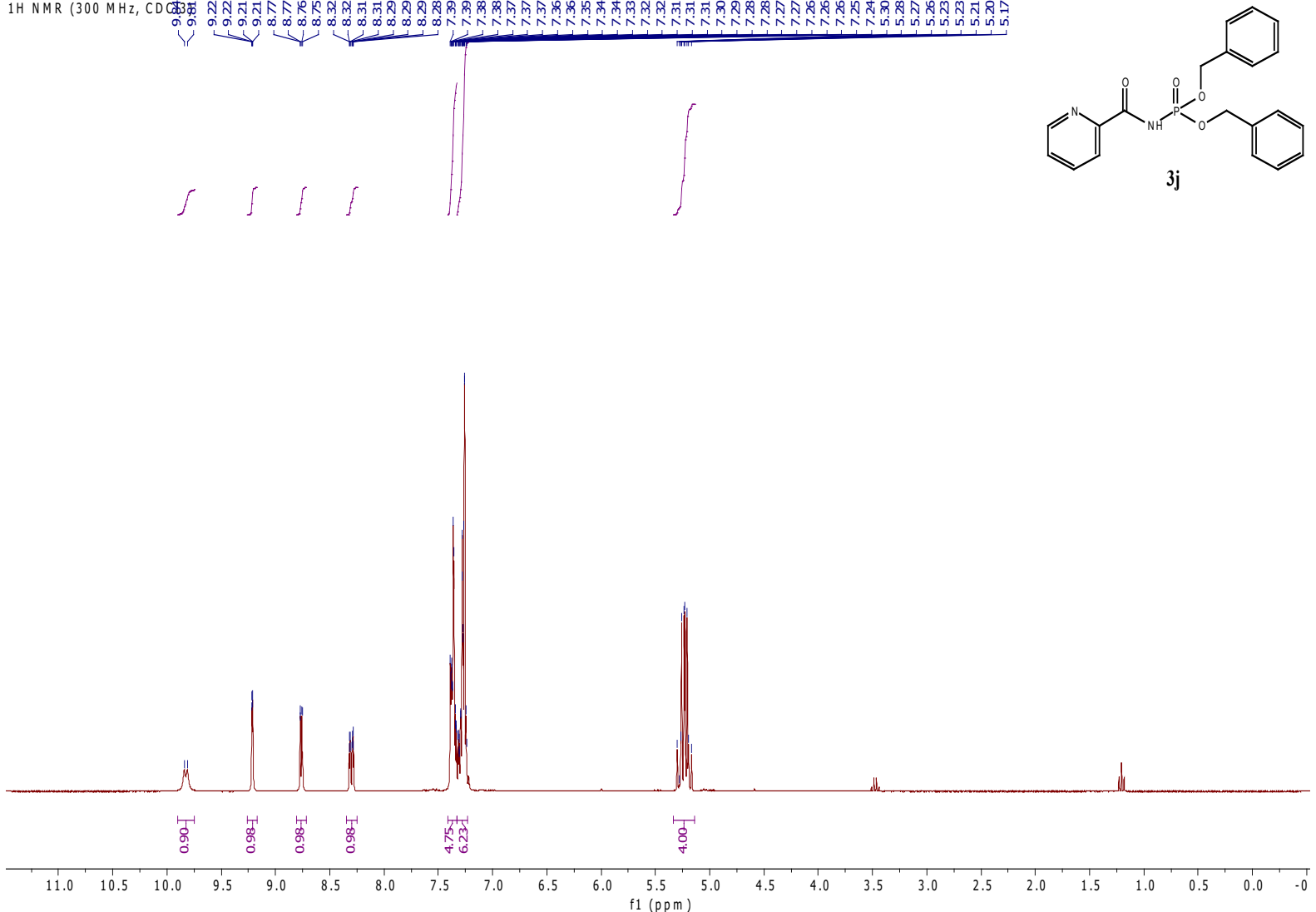

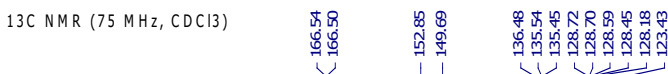

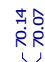
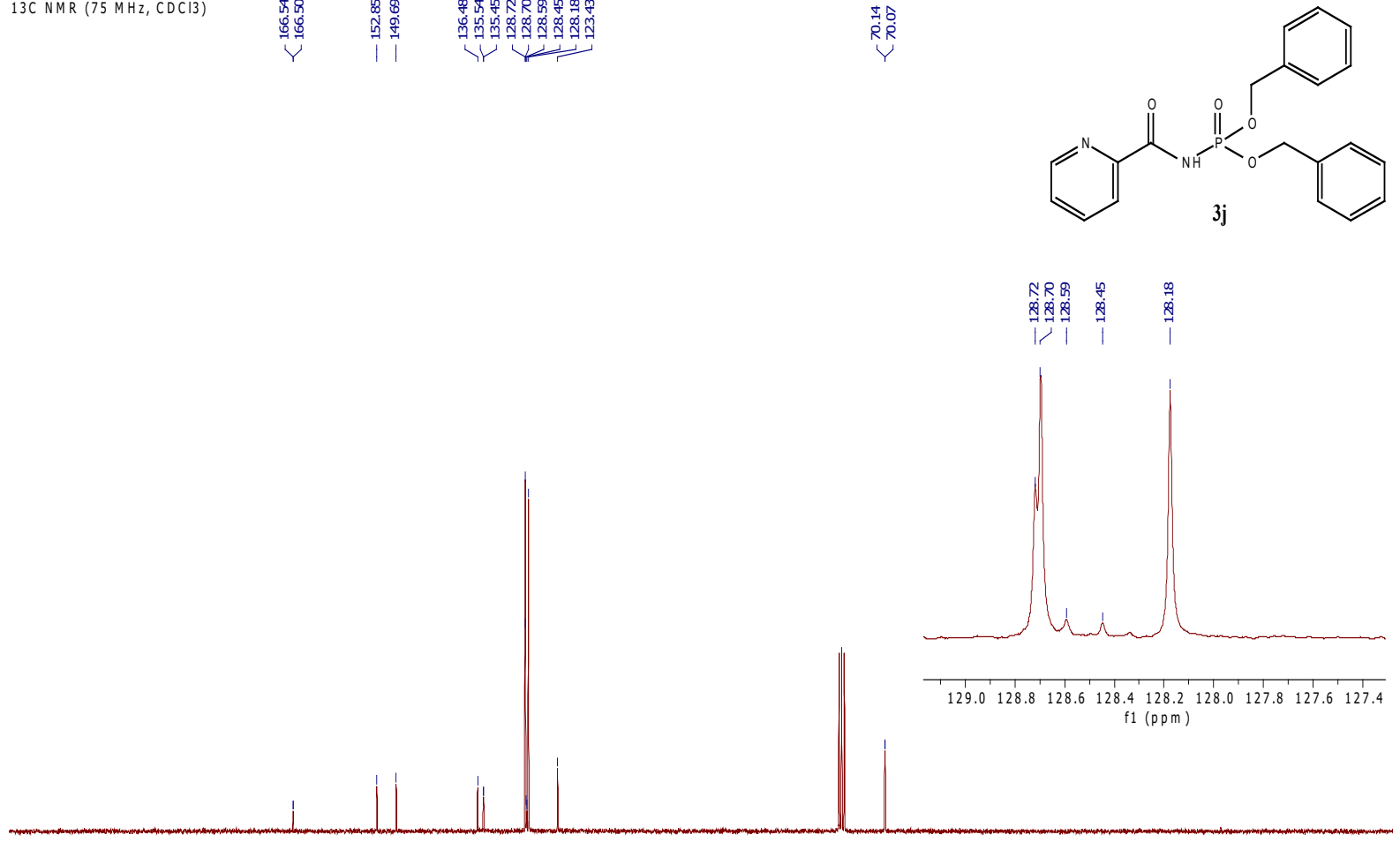

$\begin{array}{llllllllllllllllllllllll}210 & 200 & 190 & 180 & 170 & 160 & 150 & 140 & 130 & 120 & 110 & 100 & 90 & 80 & 70 & 60 & 50 & 40 & 30 & 20 & 10 & 0 & 10\end{array}$ 
31 P NMR $(121 \mathrm{MHz}, \mathrm{CDCl} 3)$<smiles>O=C(NP(=O)(OCc1ccccc1)OCc1ccccc1)c1ccccn1</smiles>
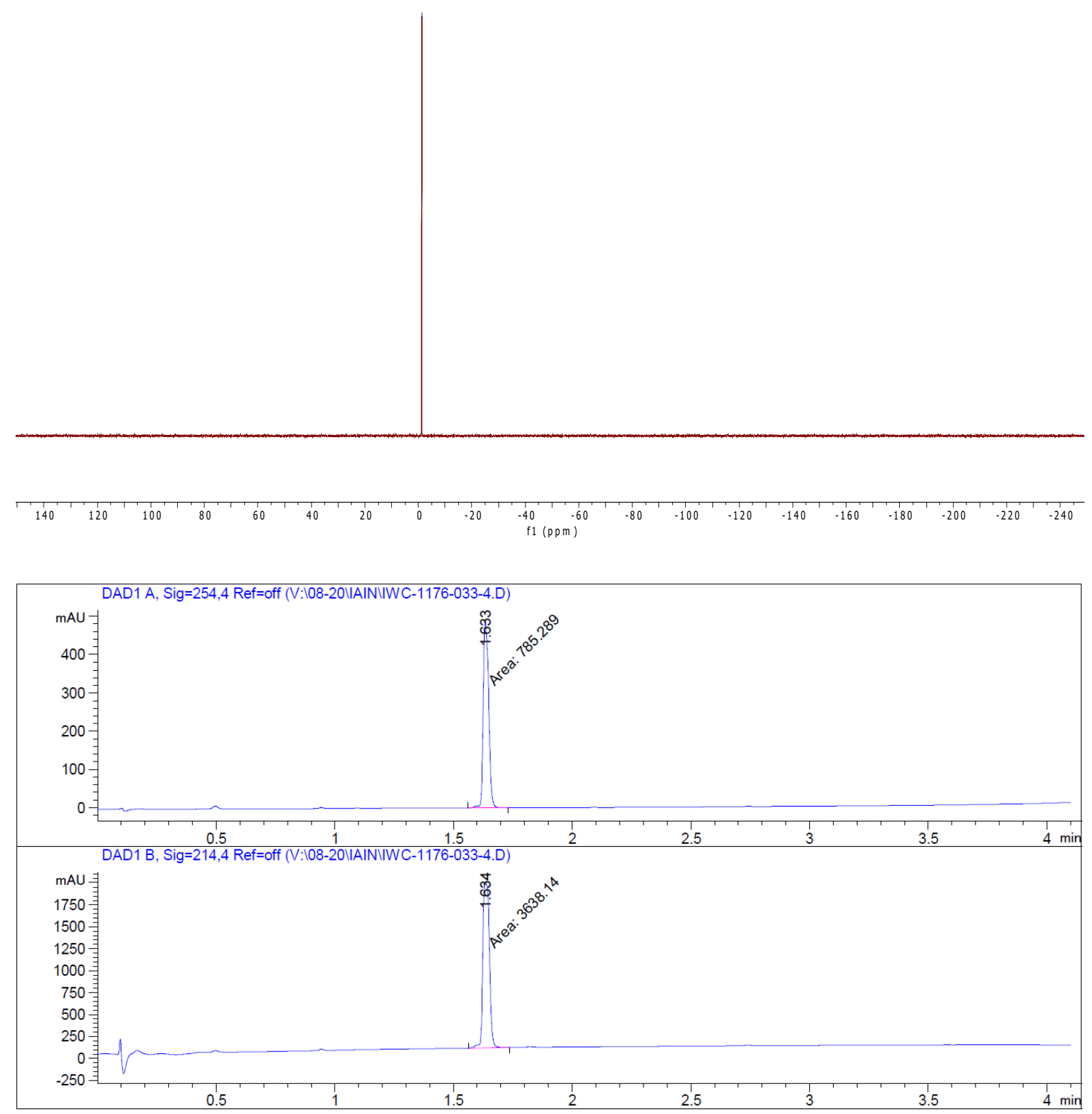
Dibenzyl $1 H$-indole-2-carbonylphosphoramidate (3k)
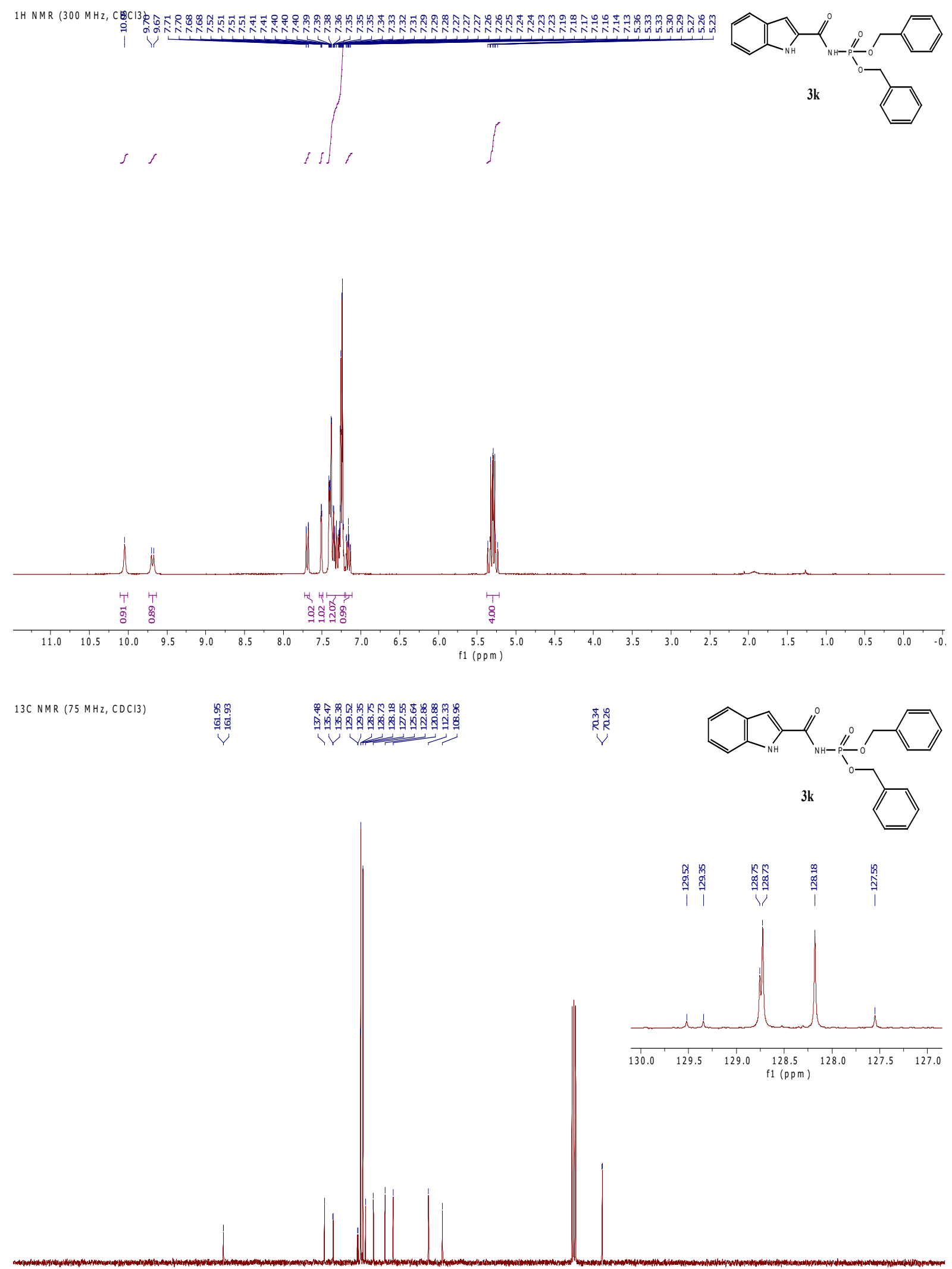

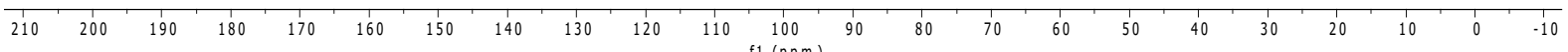



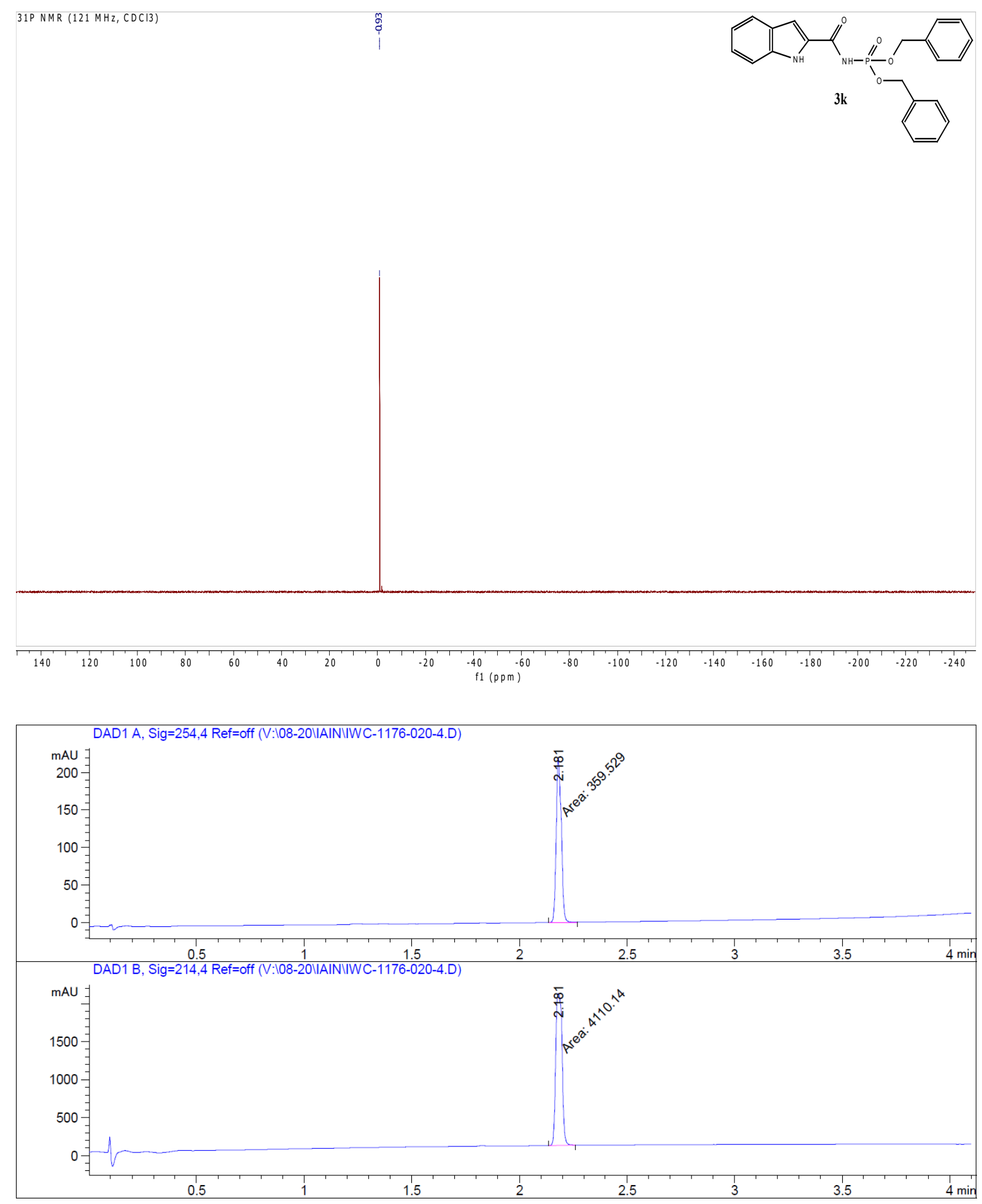
Dibenzyl $1 H$-indole-3-carbonylphosphoramidate (3I)
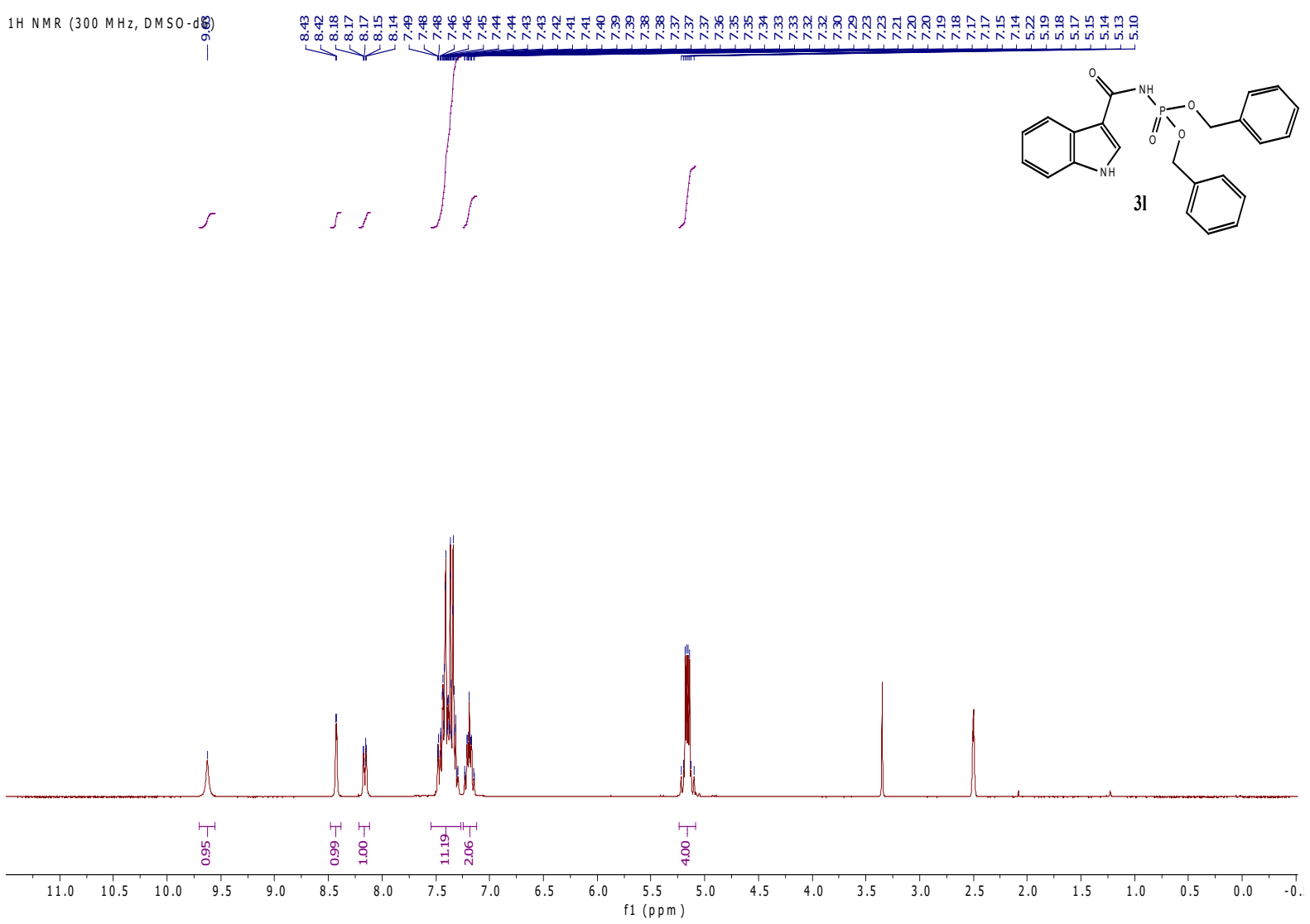

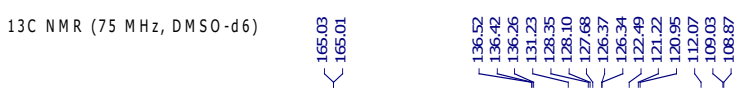

$\underbrace{1}_{\substack{9 \\ 6 \\ 6}}$
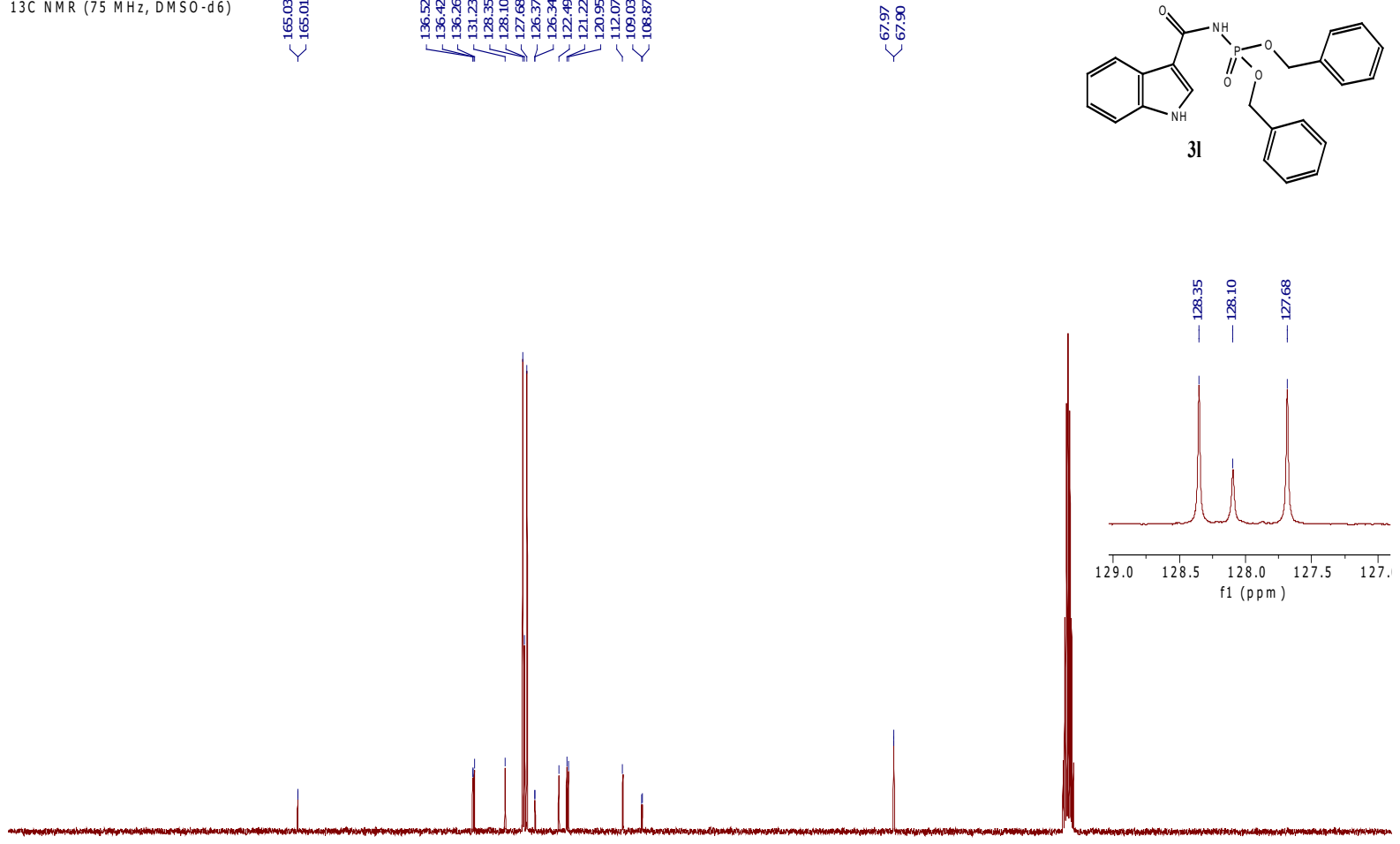

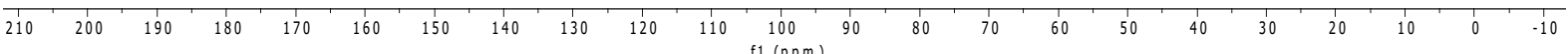


31P NMR (121 MHz, DMSO-d6)
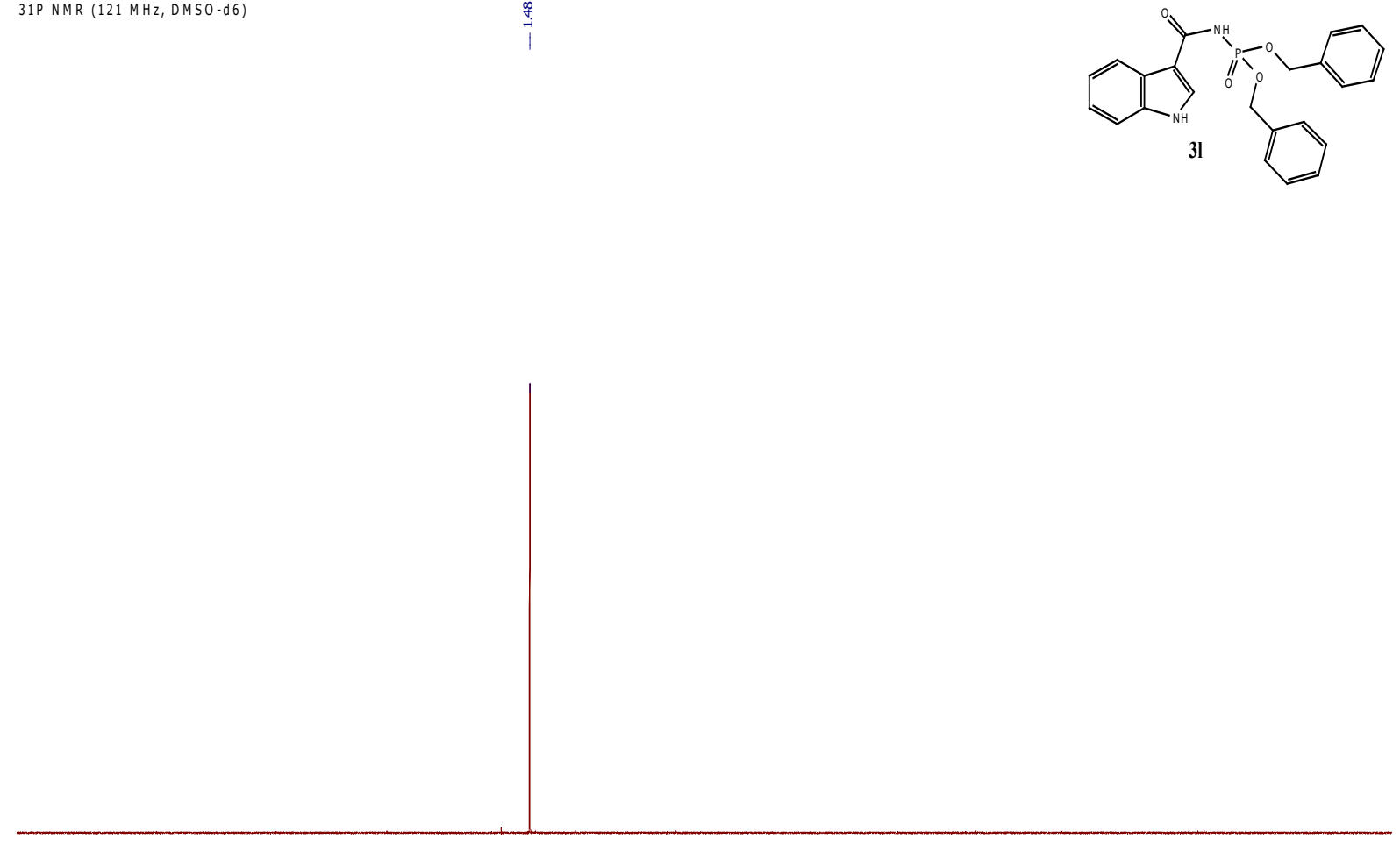

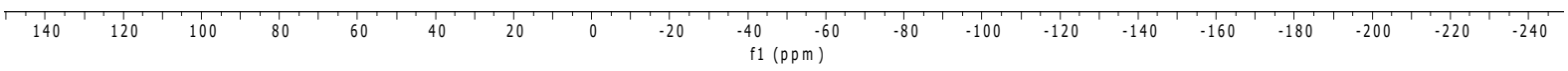

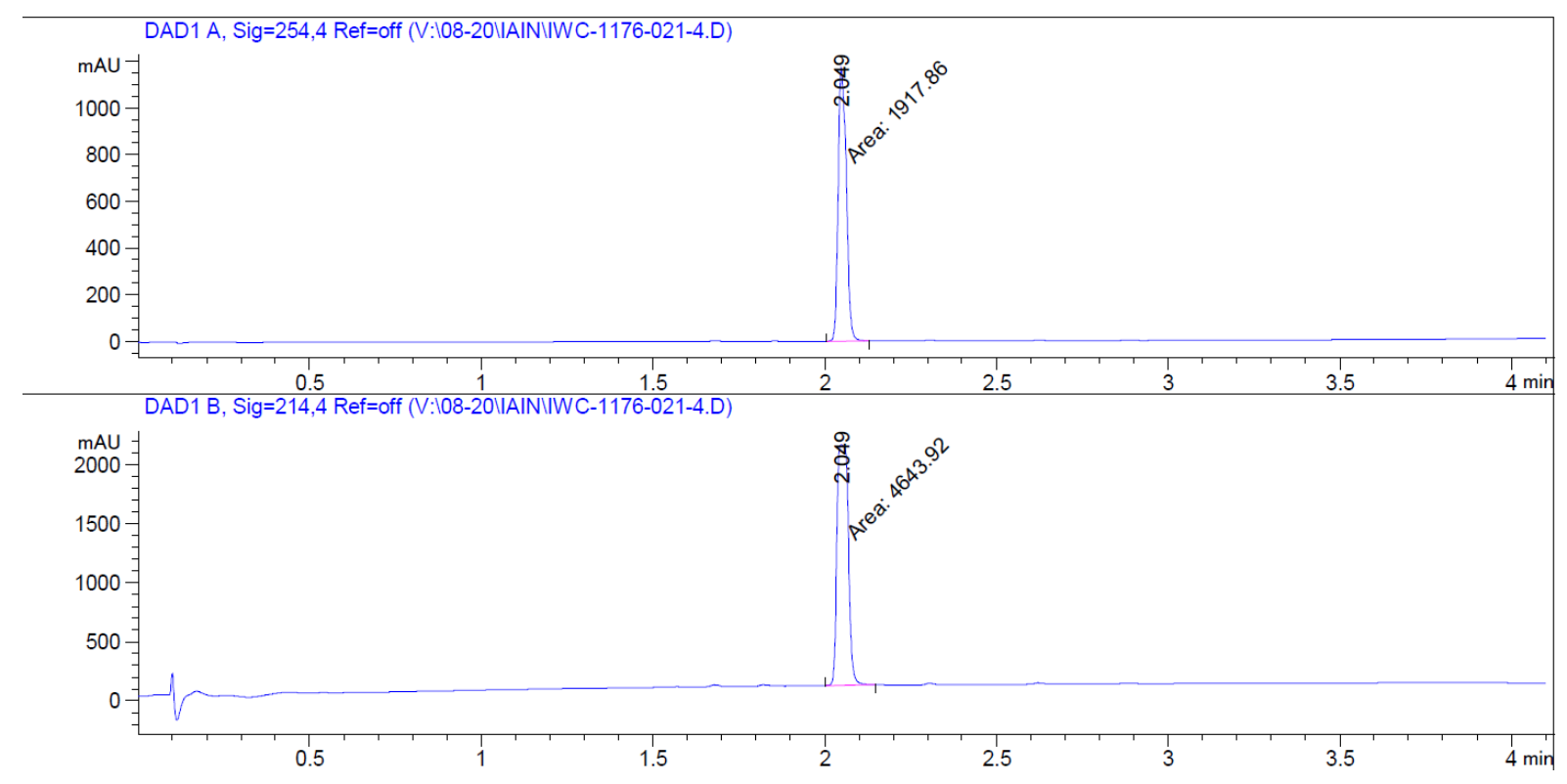


Dibenzyl (4-nitrobenzoyl)phosphoramidate (3m)

1H NMR 300 MHz, CDClöt
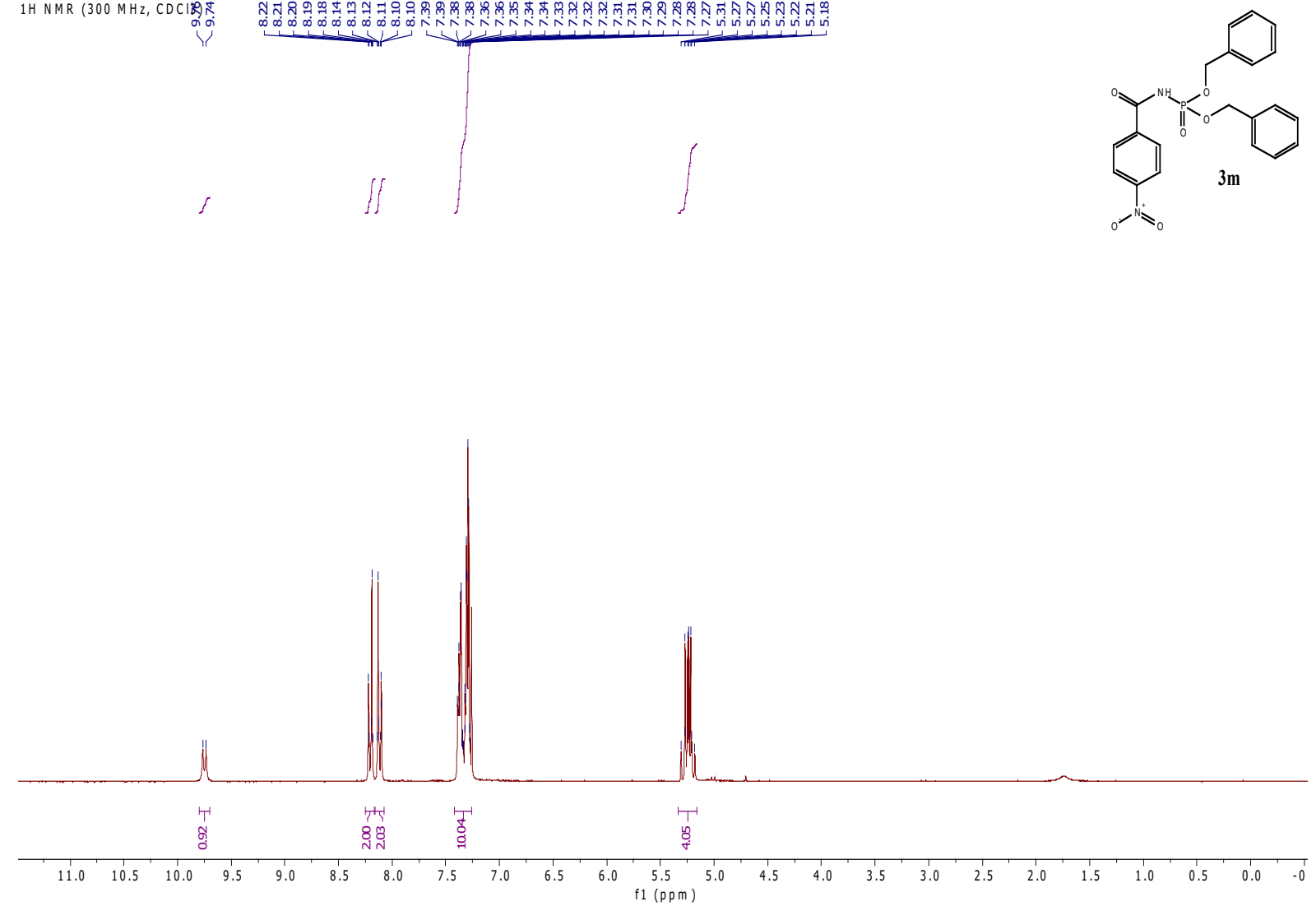

$13 \mathrm{CNMR}(75 \mathrm{MHz}, \mathrm{CDCl}) \quad$ 范

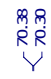

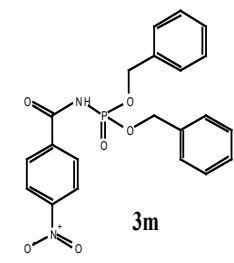

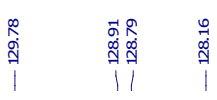

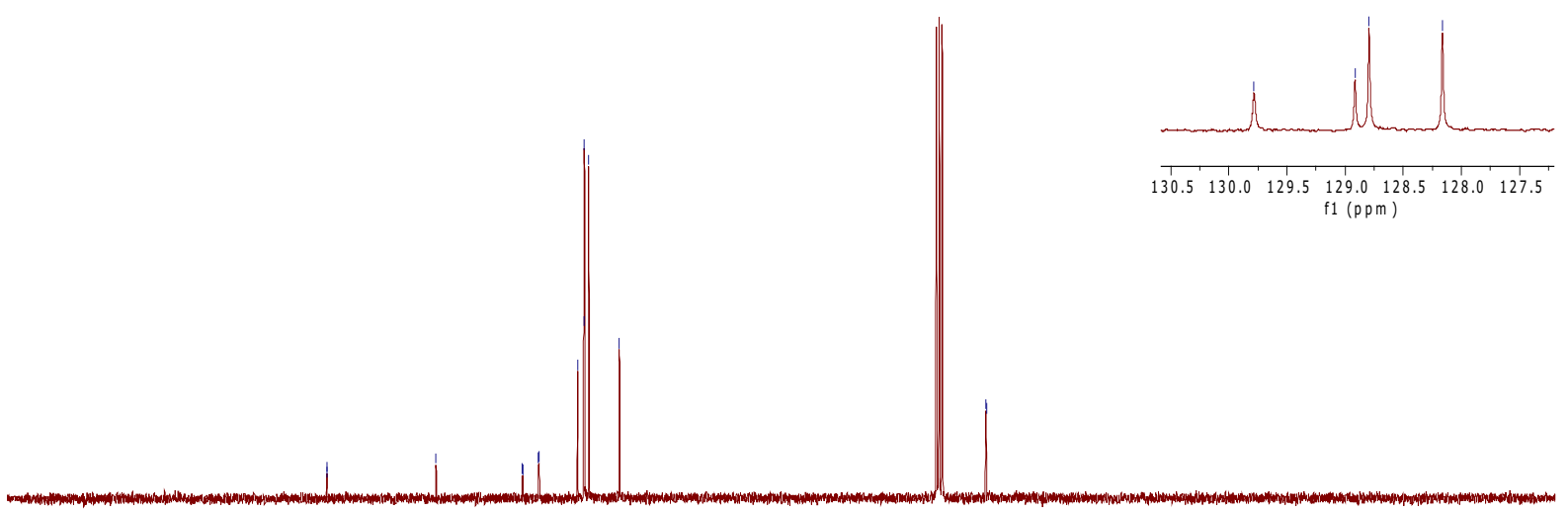

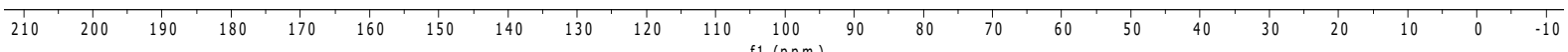



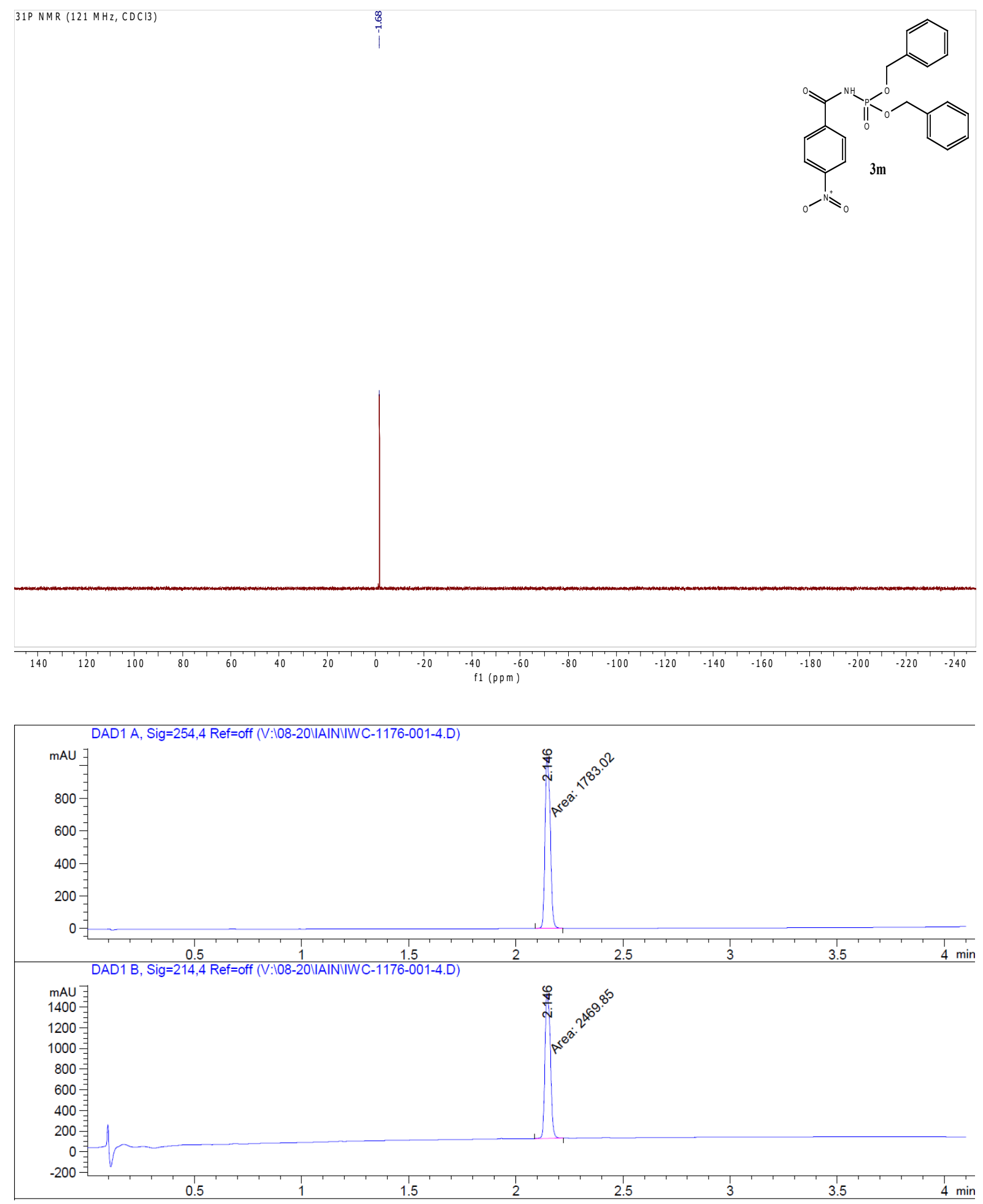
Dibenzyl (4-(dimethylamino)benzoyl)phosphoramidate (3n)
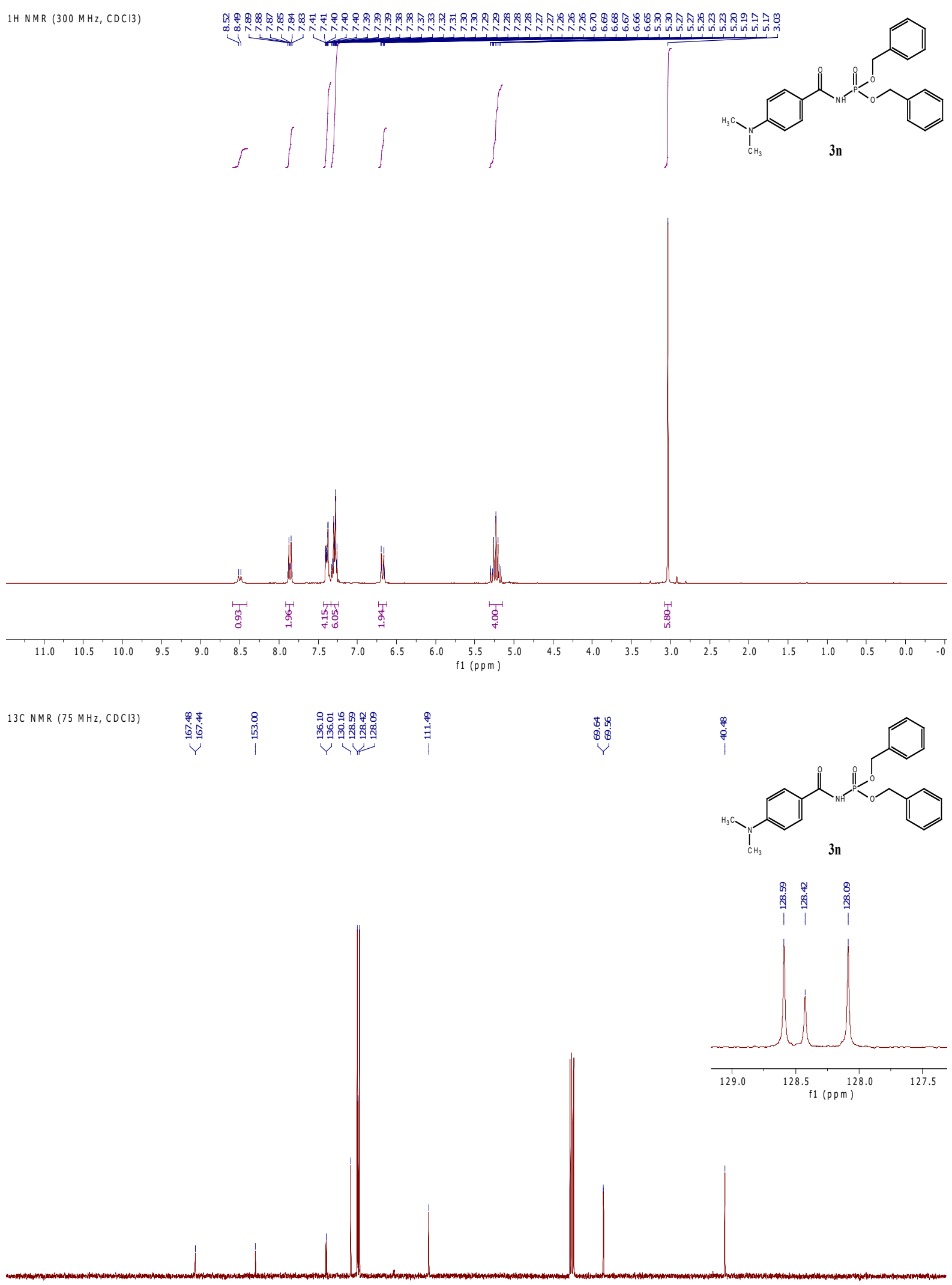

$\begin{array}{llllllllllllllllllllllllll}1 & 1 \\ 210 & 200 & 190 & 180 & 170 & 160 & 150 & 140 & 130 & 120 & 110 & 100 & 90 & 80 & 70 & 60 & 50 & 40 & 30 & 20 & 10 & 0 & -10\end{array}$ 

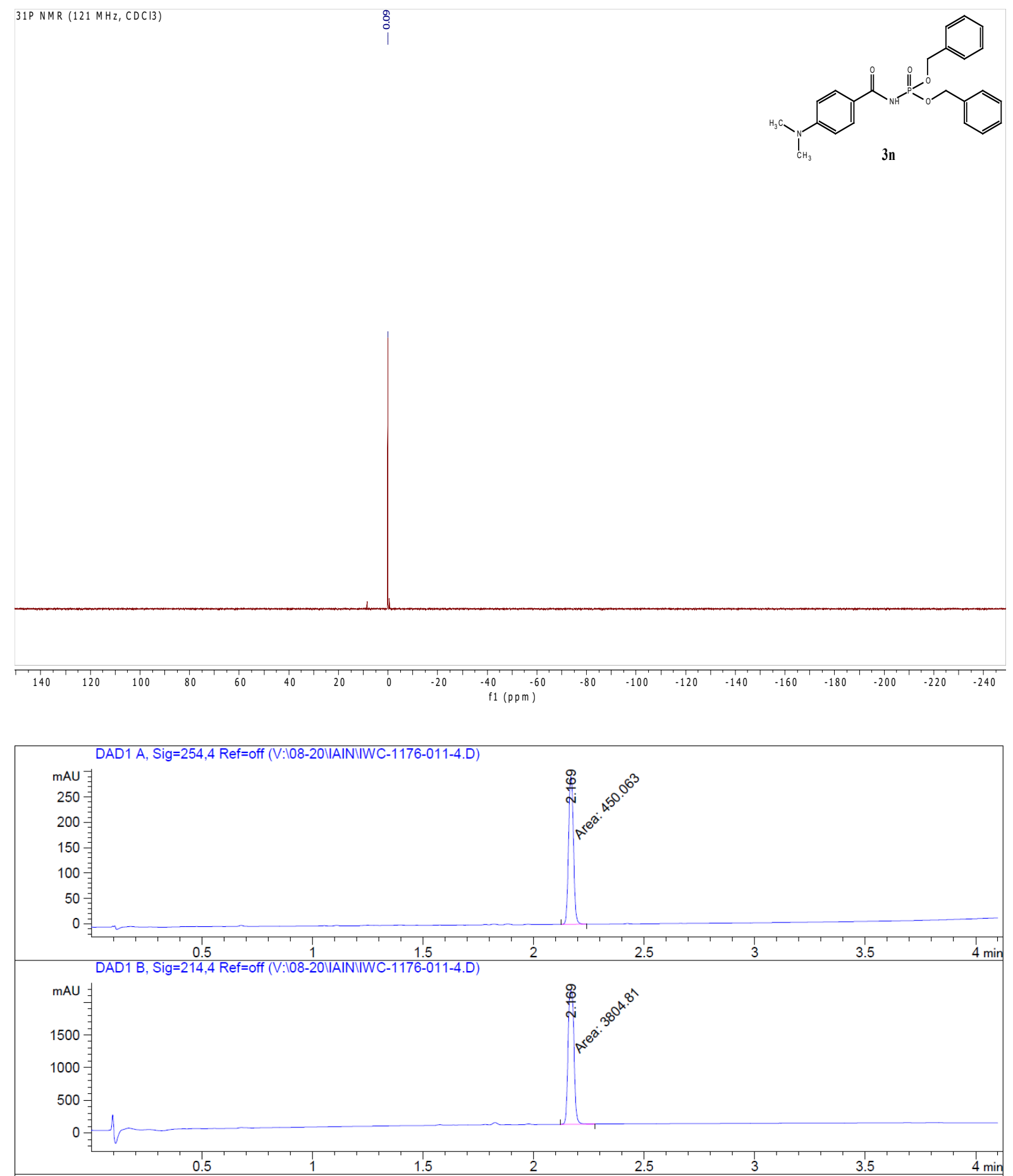


\section{Dibenzyl (3-acetylbenzoyl)phosphoramidate (3p)}

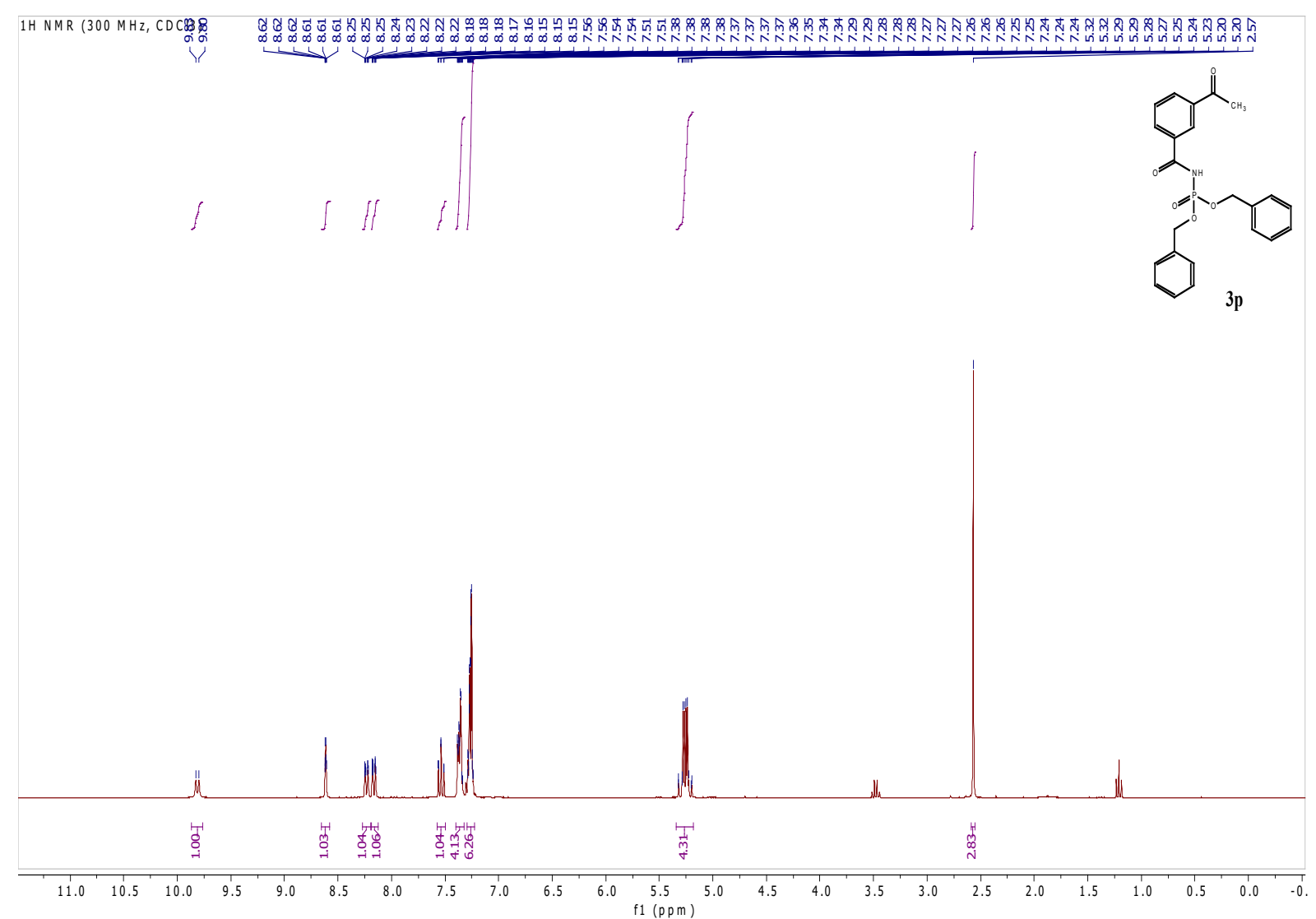

13C NMR $(75 \mathrm{MHz}, \mathrm{CDCl3}) \quad$ Y

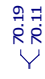
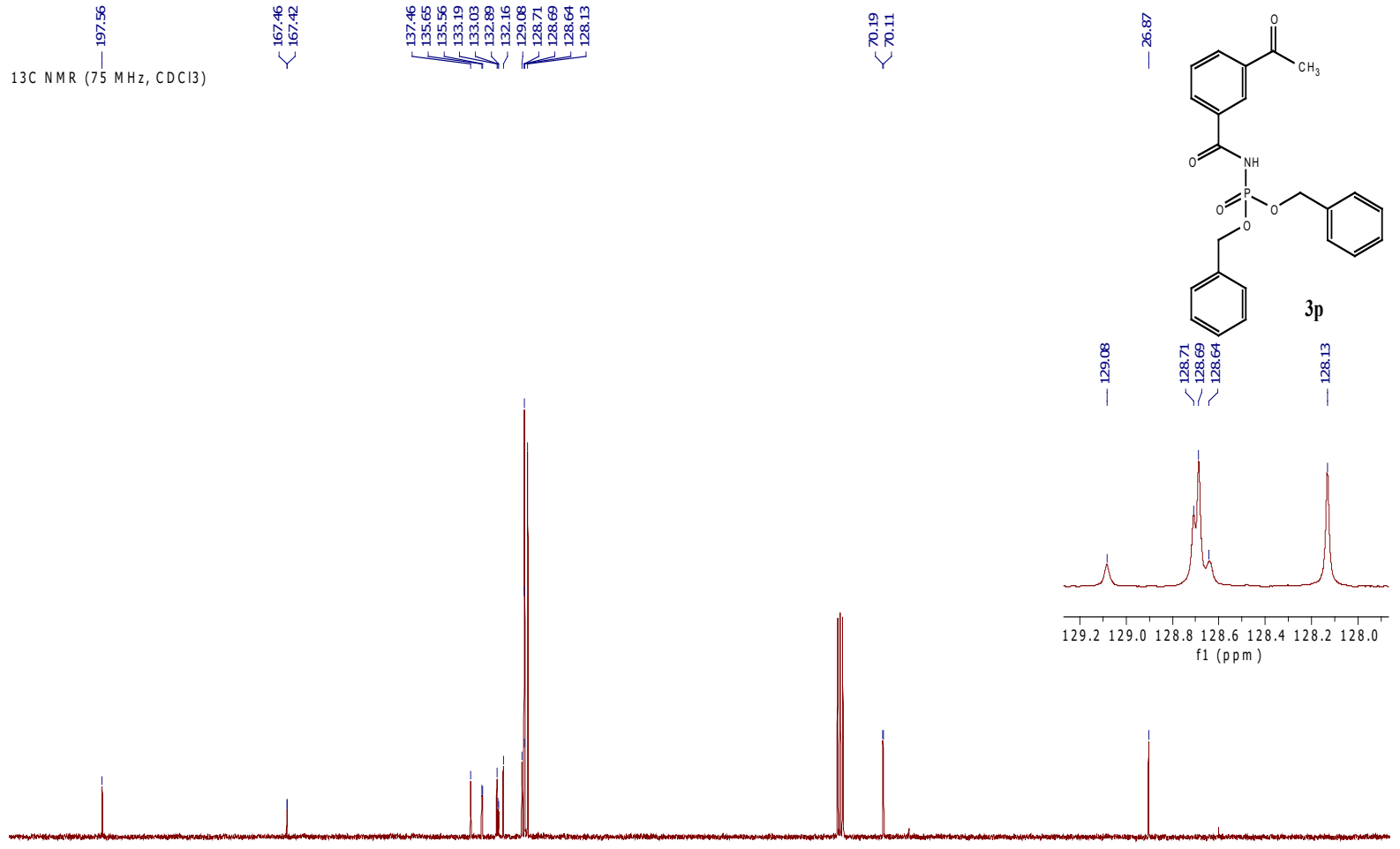

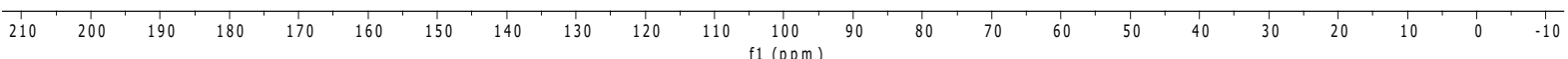



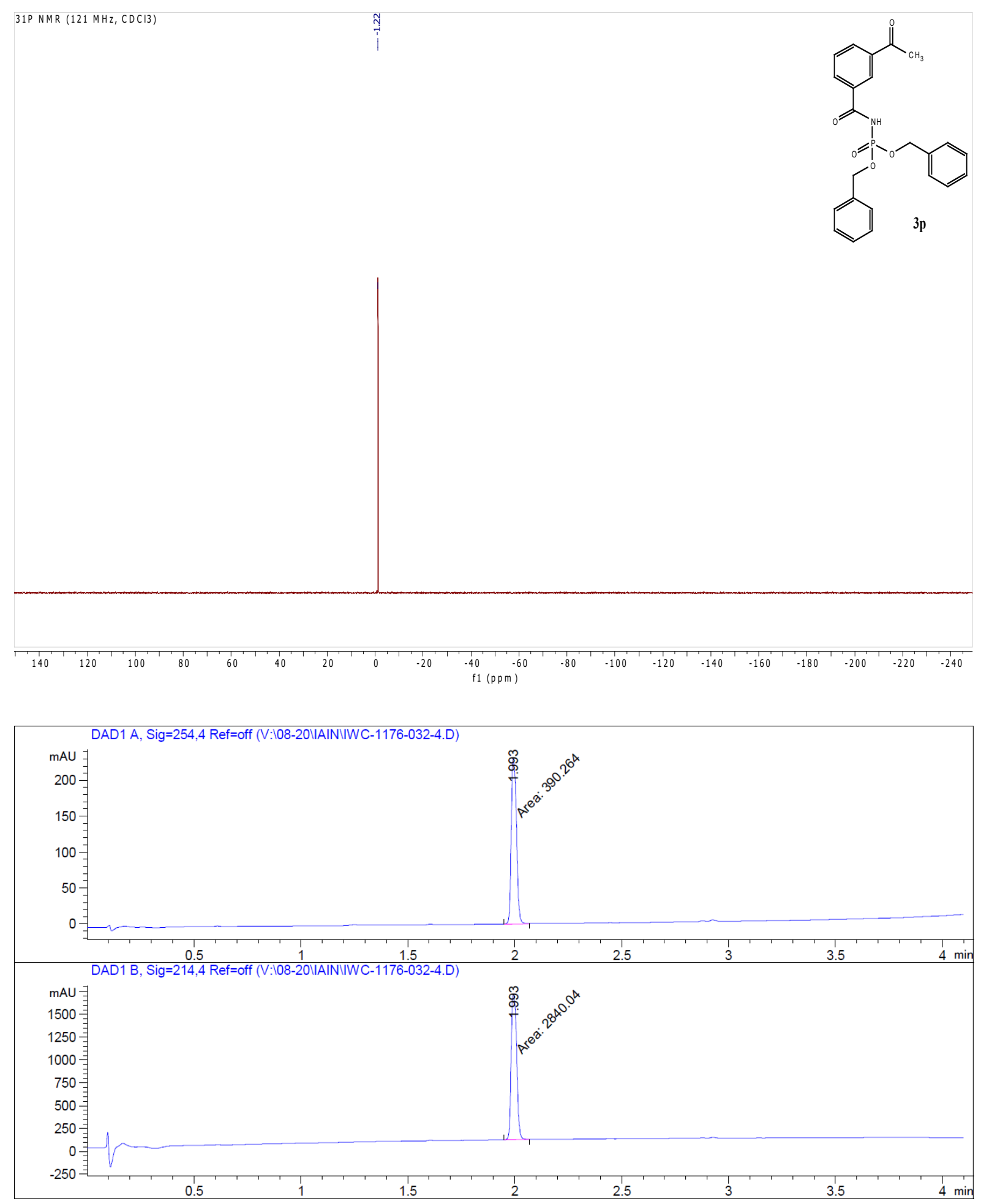
Dibenzyl (4-benzoylbenzoyl)phosphoramidate (3q)
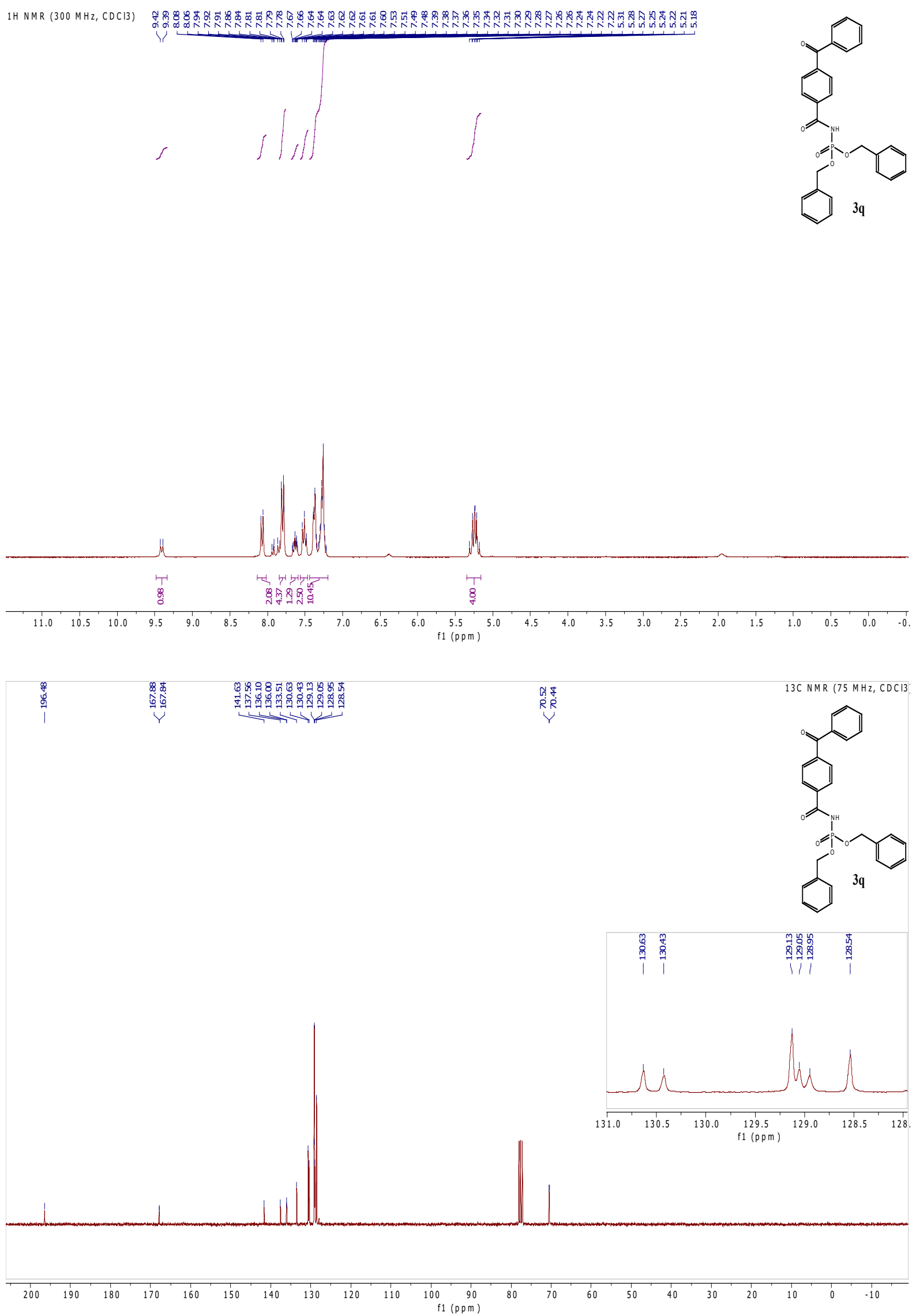


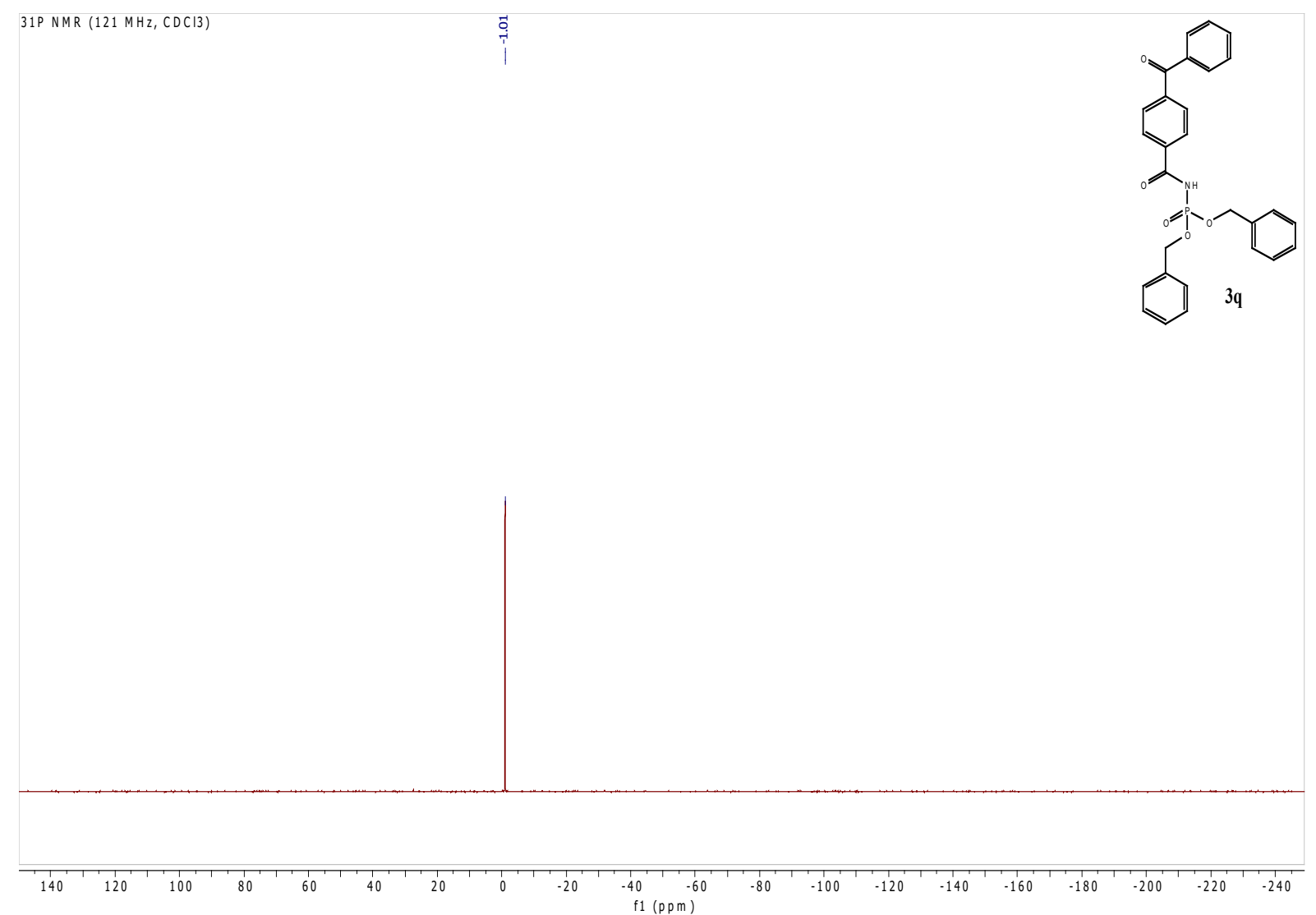

DAD1 A, Sig=254,4 Ref=off (V:111-20VIAINliwc-1176-031-4.d)

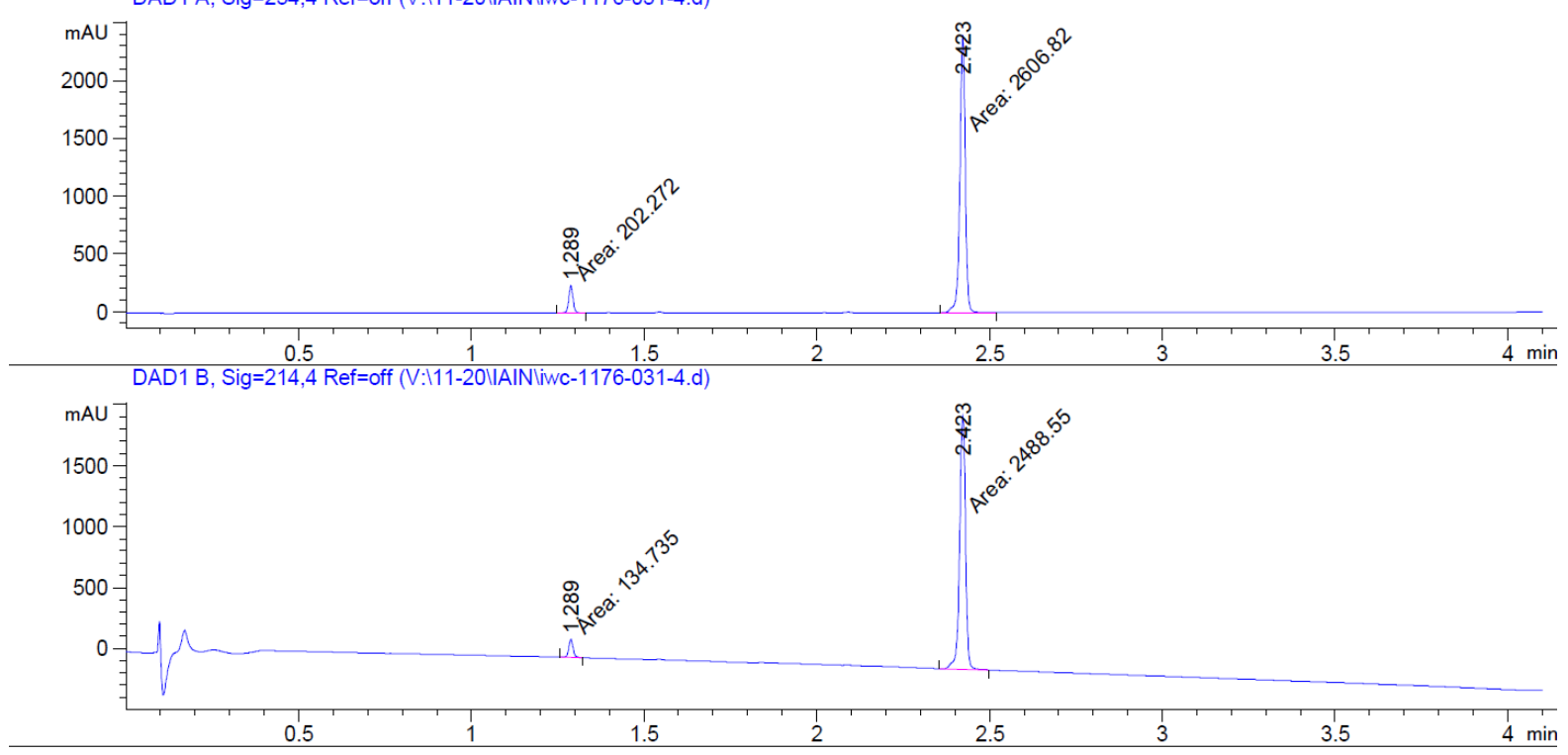


Dibenzyl (4-(methylsulfonyl)benzoyl)phosphoramidate (3r)
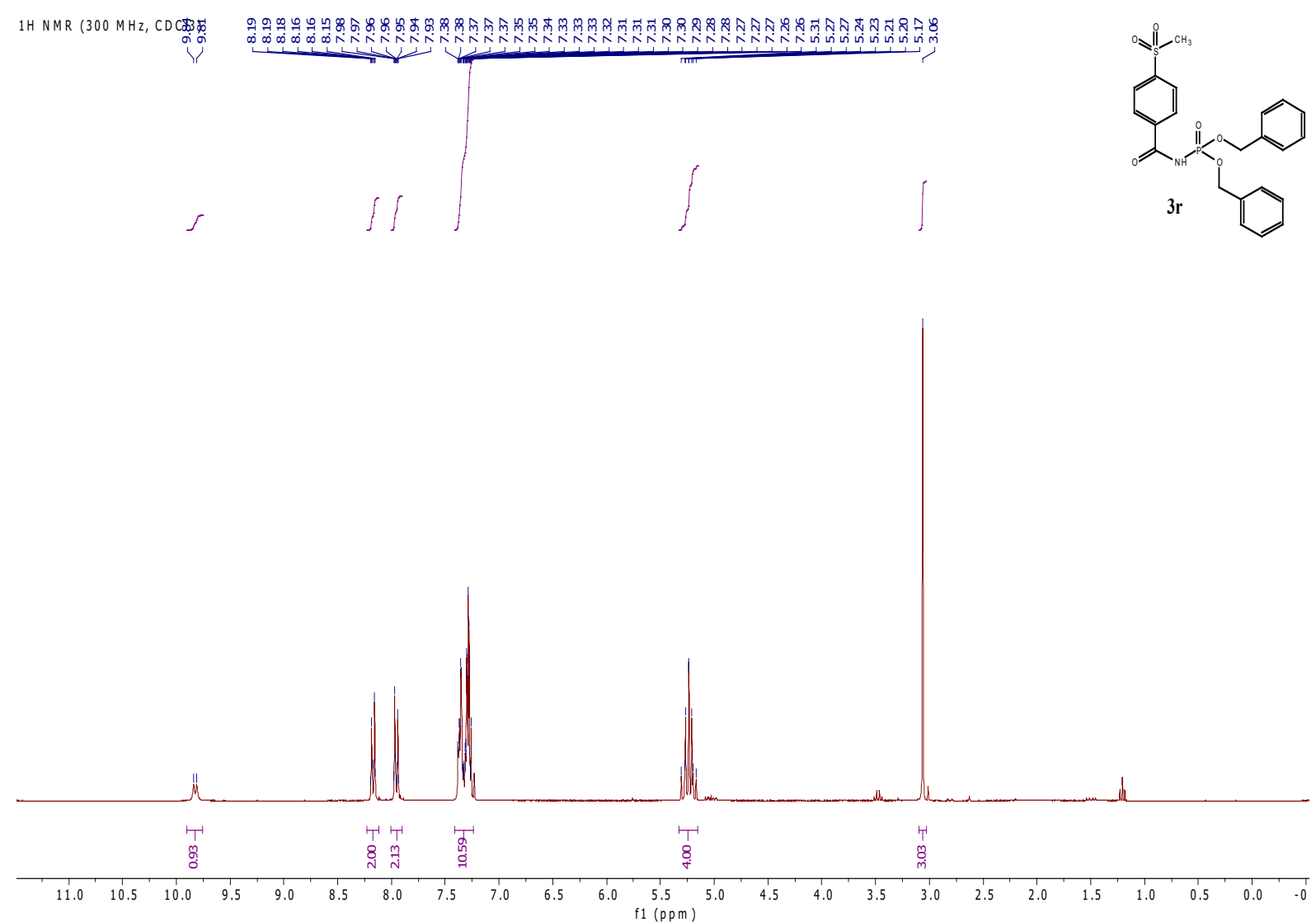

13C NMR $(75 \mathrm{MHz}, \mathrm{CDCl} 3)$
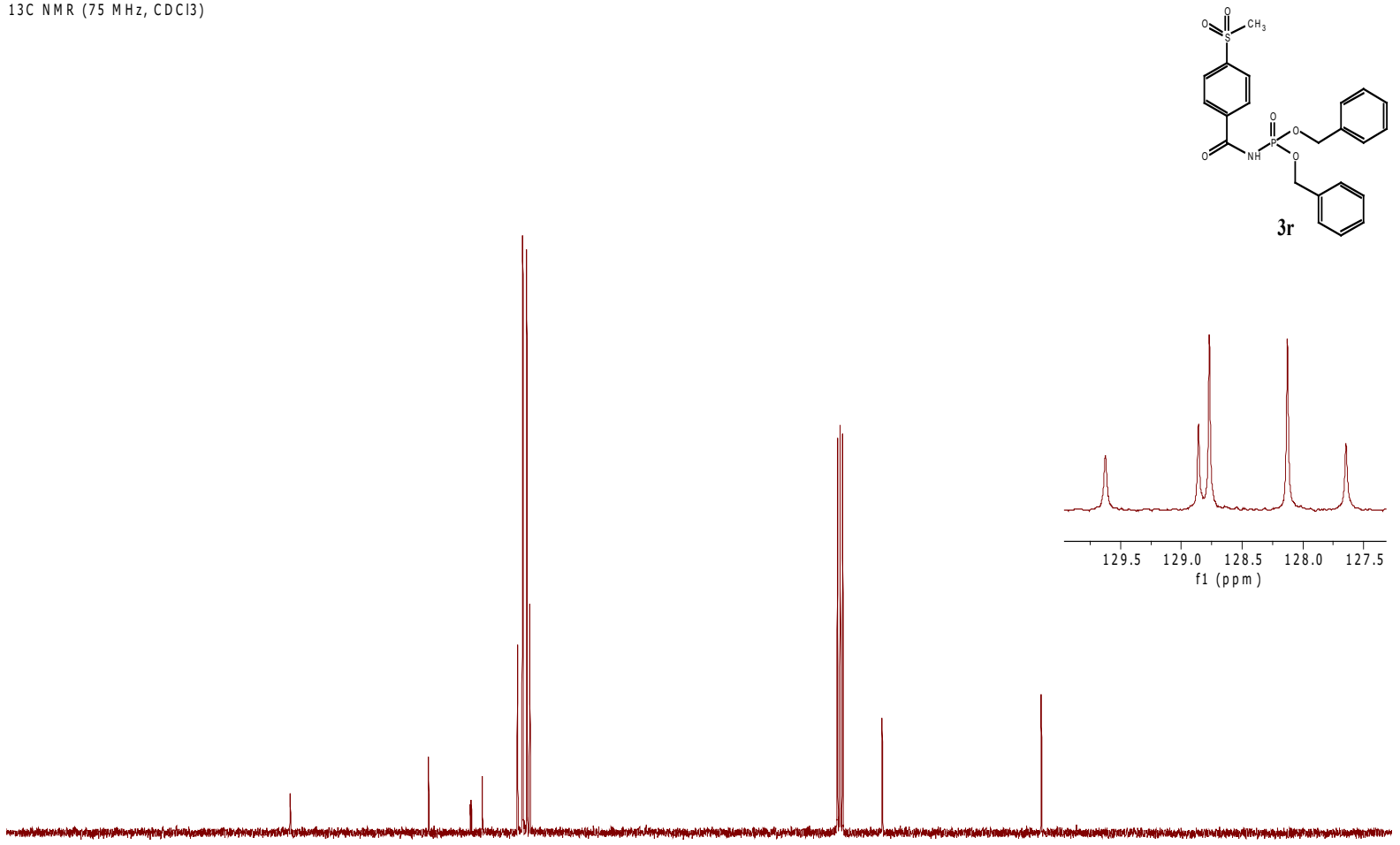

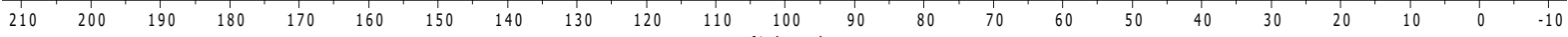


31 P NMR $(121 \mathrm{MHZ}, \mathrm{CDCl} 3)$
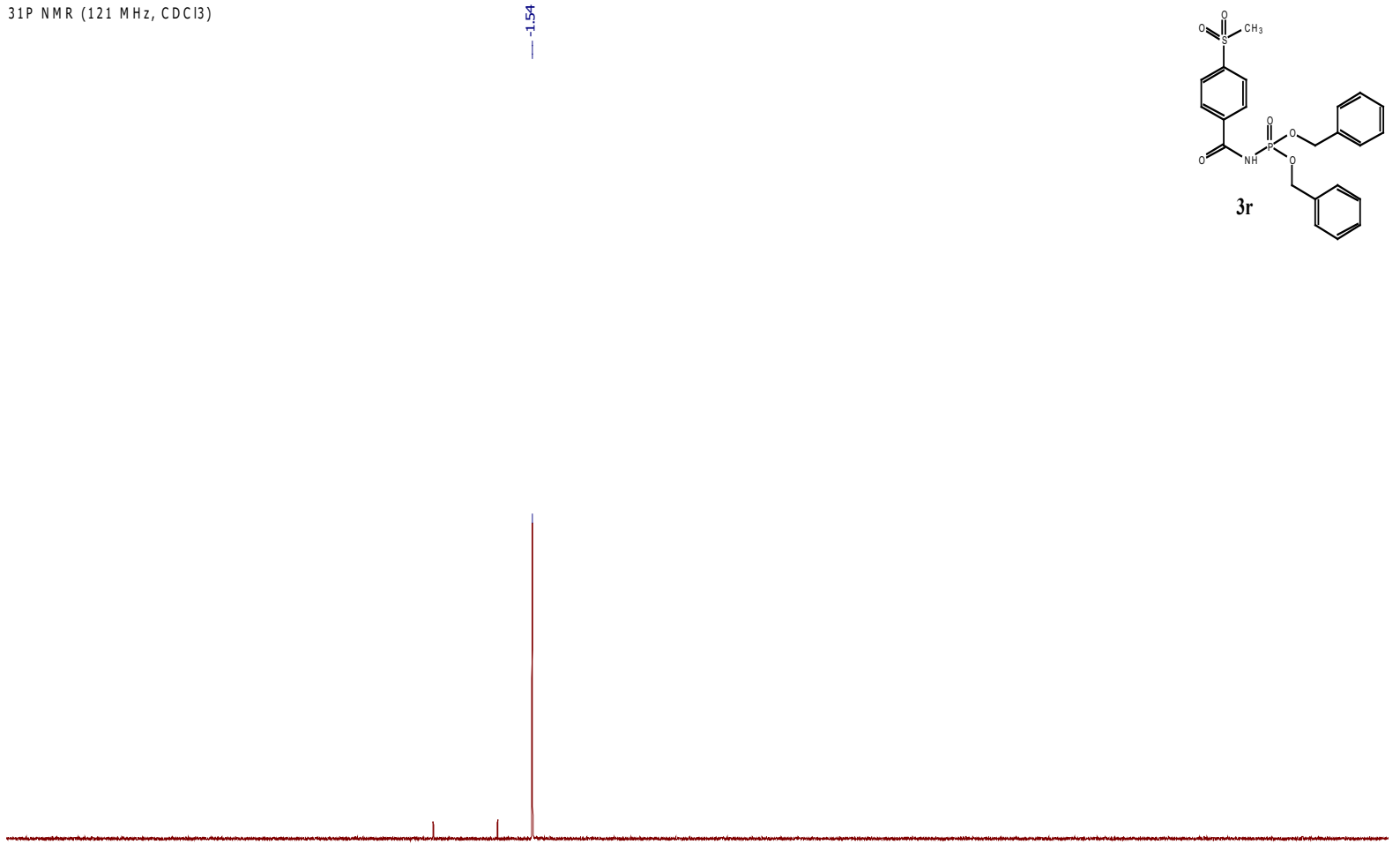

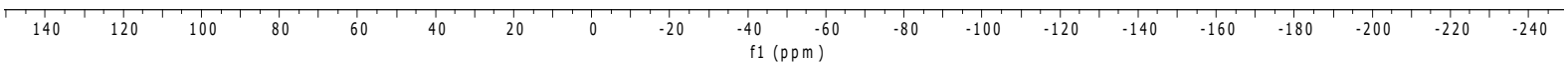

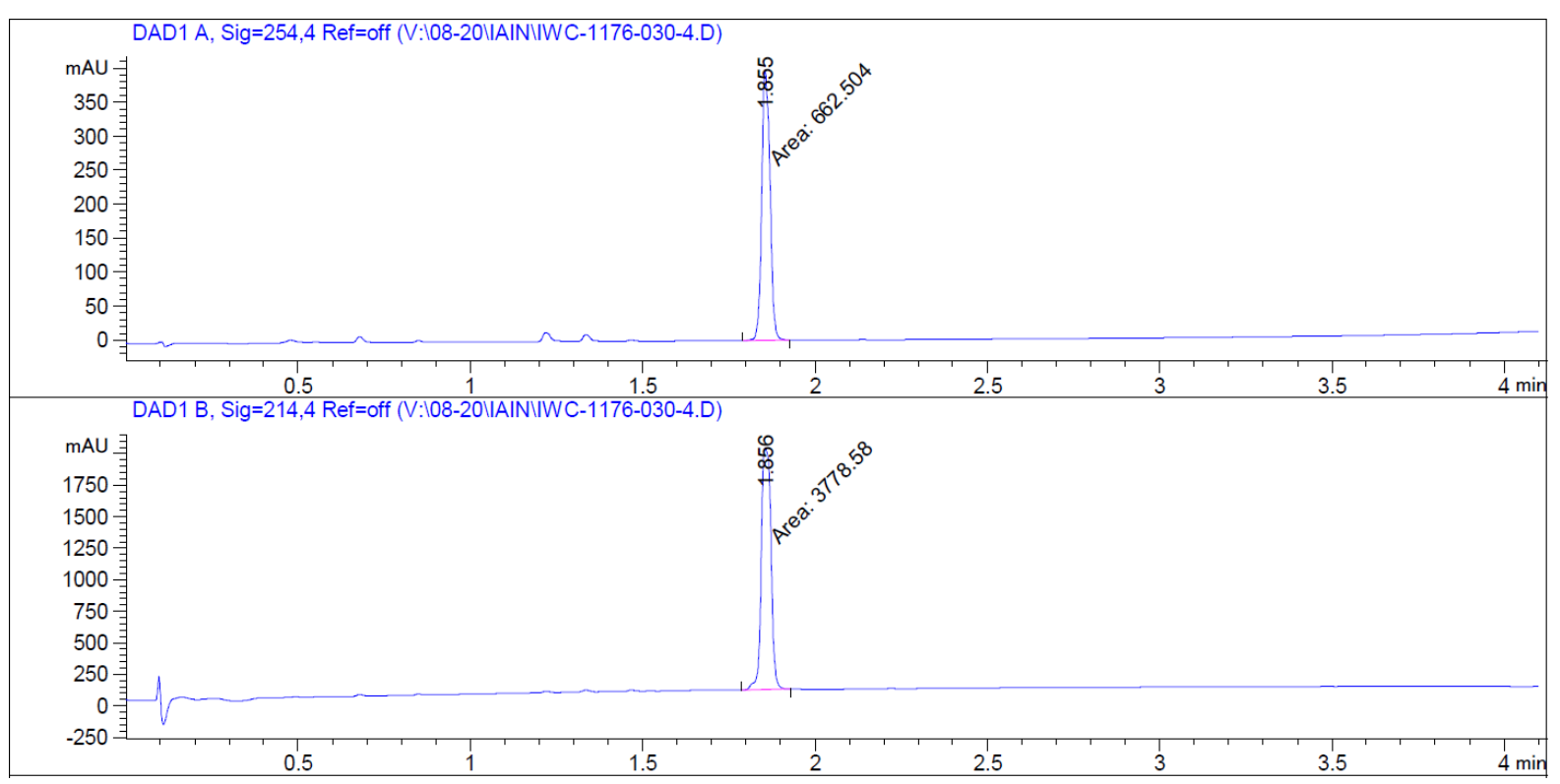


Dibenzyl (3-(methylcarboxy)benzoyl)phosphoramidate (3s)

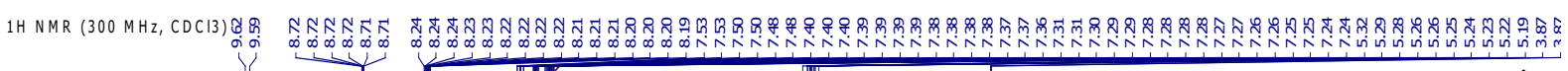
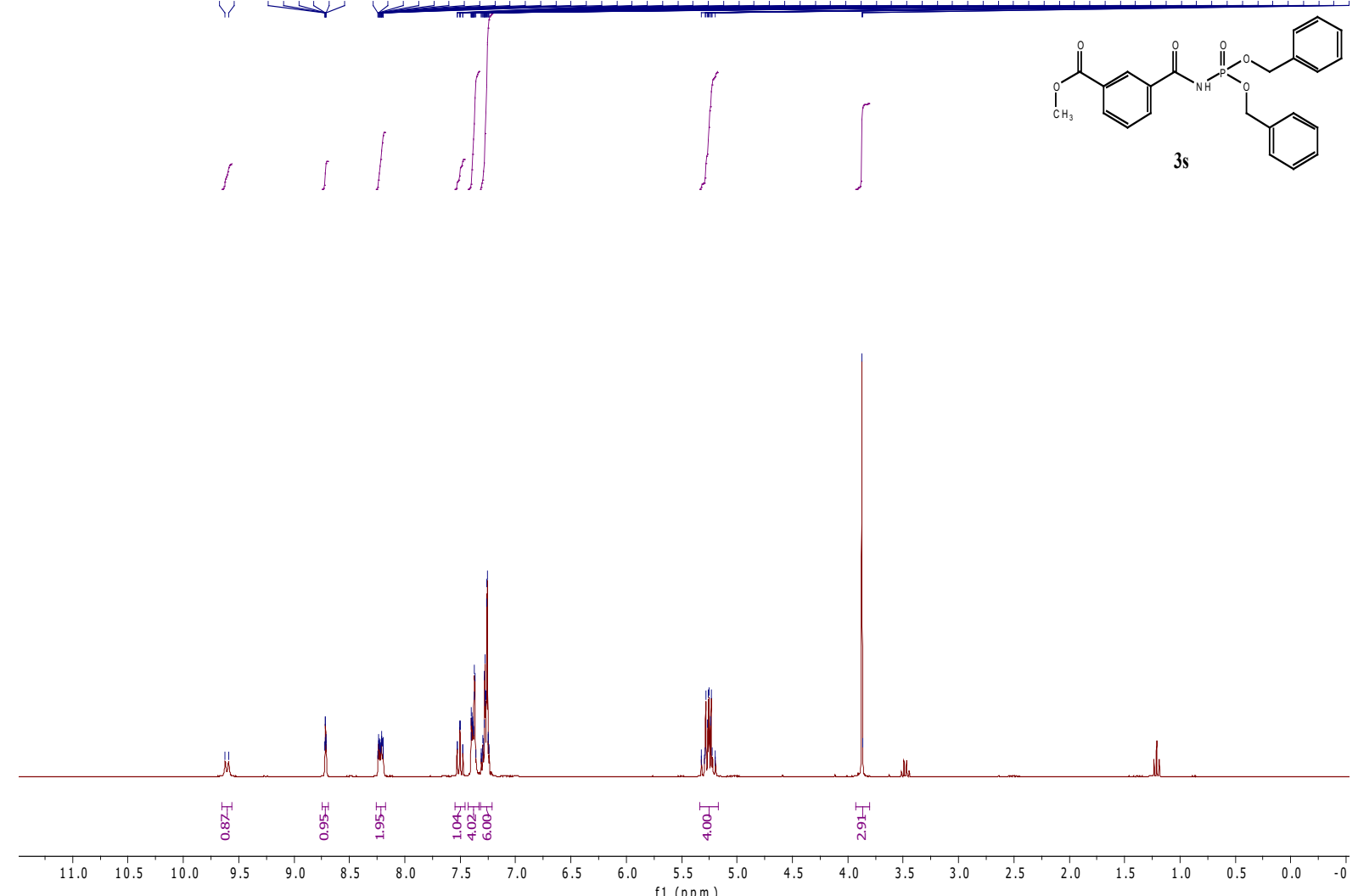

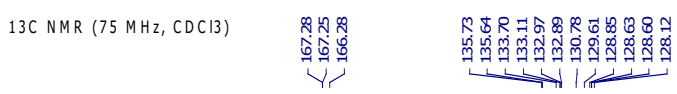

8.8
$\substack{8 \\ 8}$
$Y$

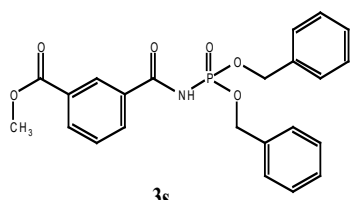

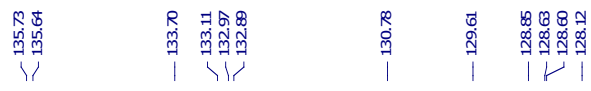

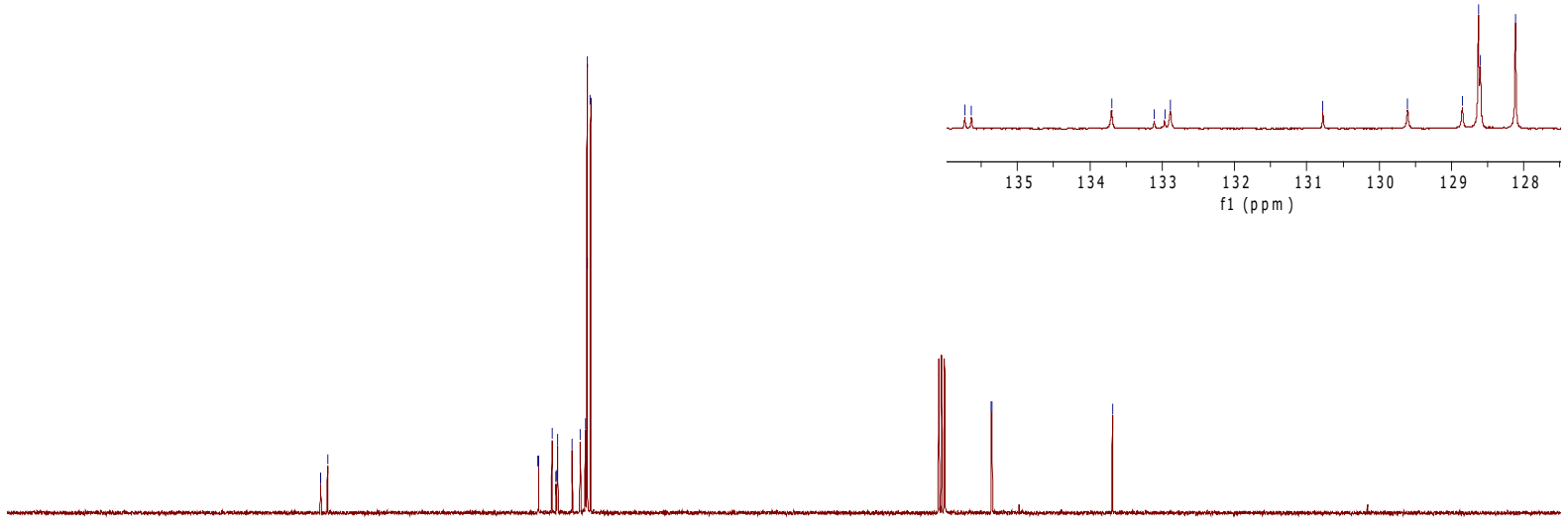

$\begin{array}{rllllllllllllllllllllllll}1 & 1 \\ 210 & 200 & 190 & 180 & 170 & 160 & 150 & 140 & 130 & 120 & 110 & 100 & 90 & 80 & 70 & 60 & 50 & 40 & 30 & 20 & 10 & 0 & -10\end{array}$ 

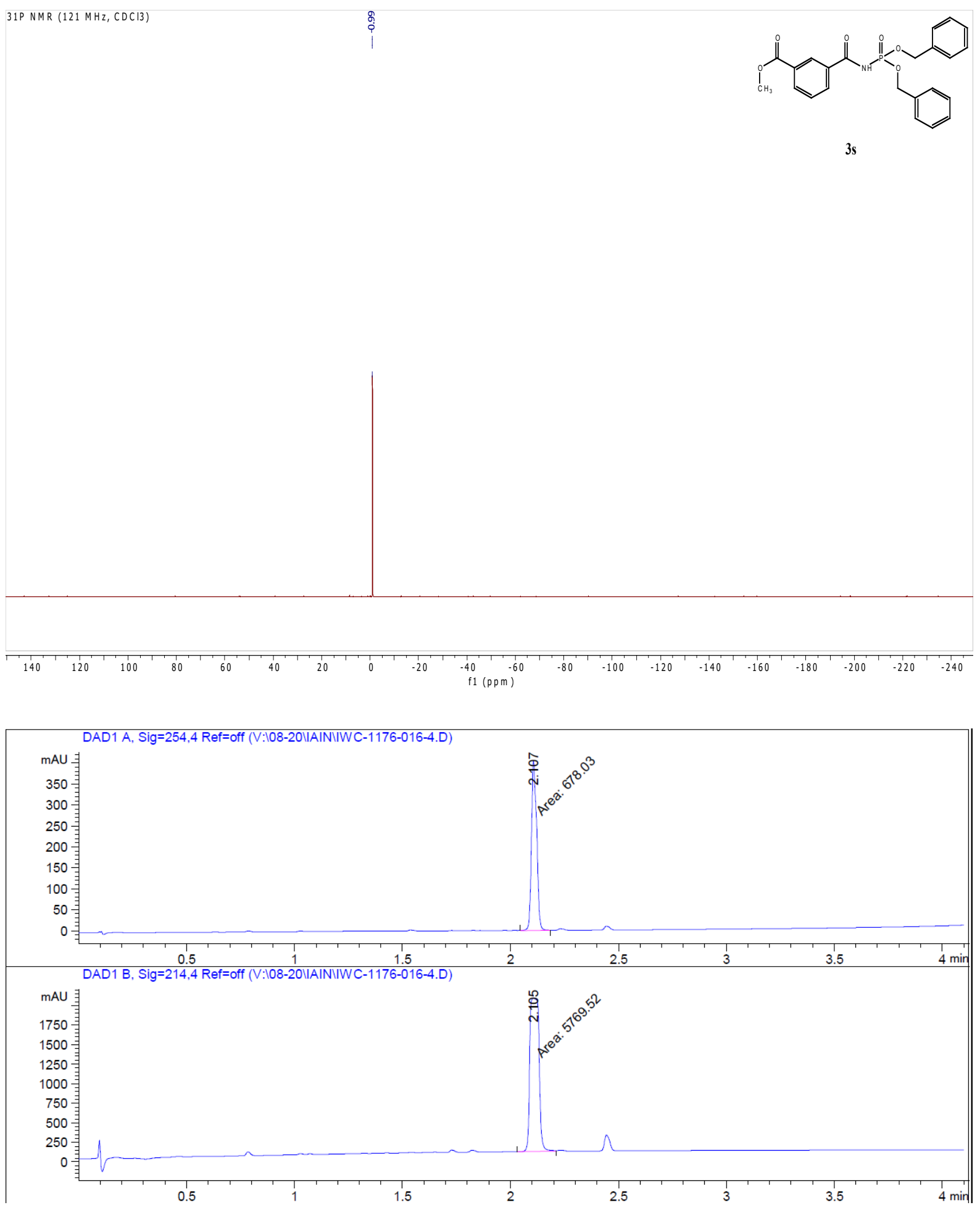
Dibenzyl (4-methoxybenzoyl)phosphoramidate (3t)

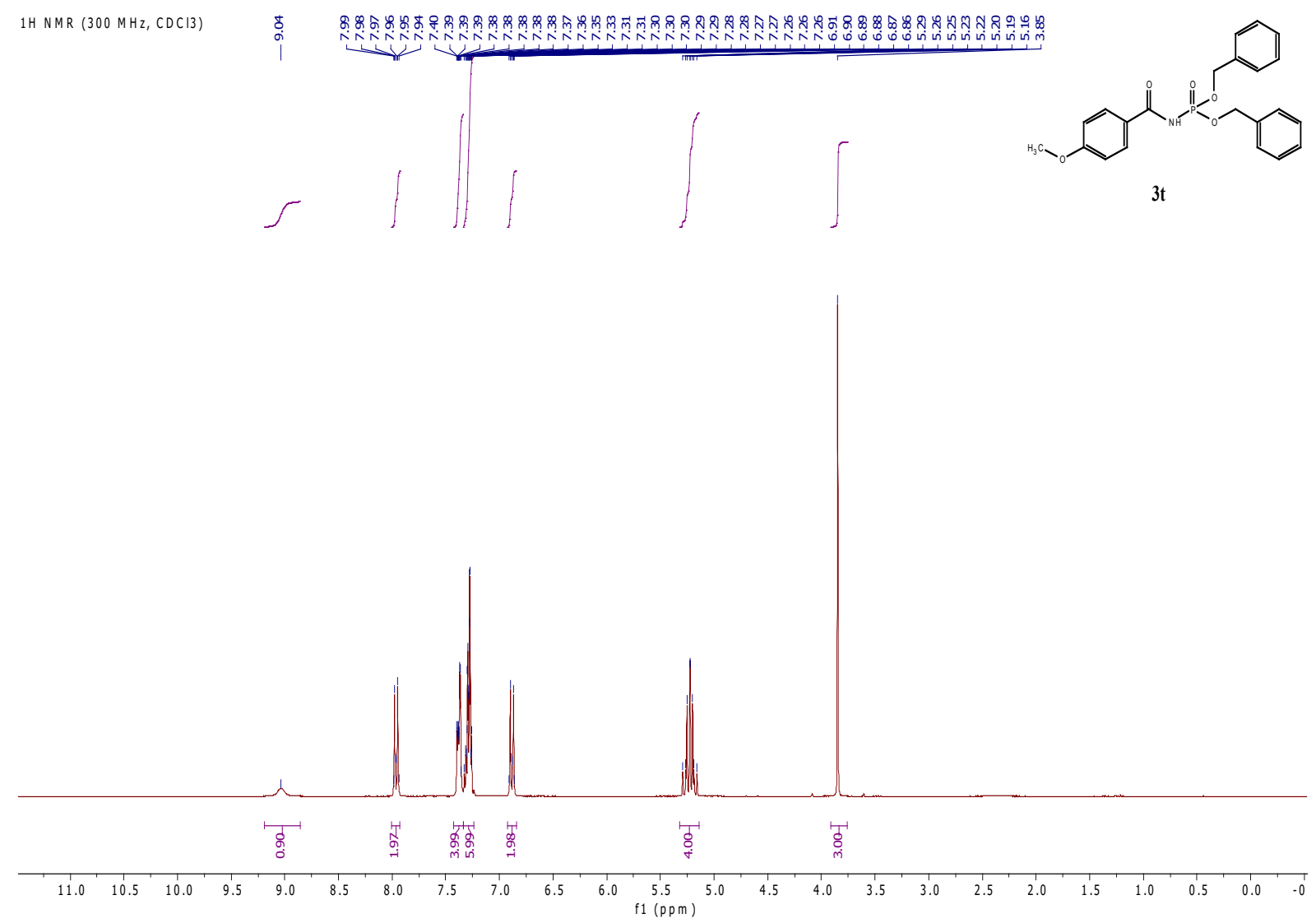

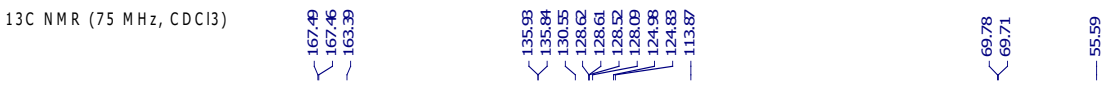

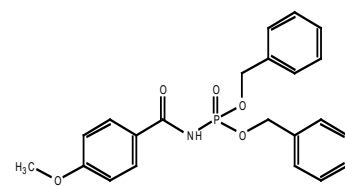

$3 t$
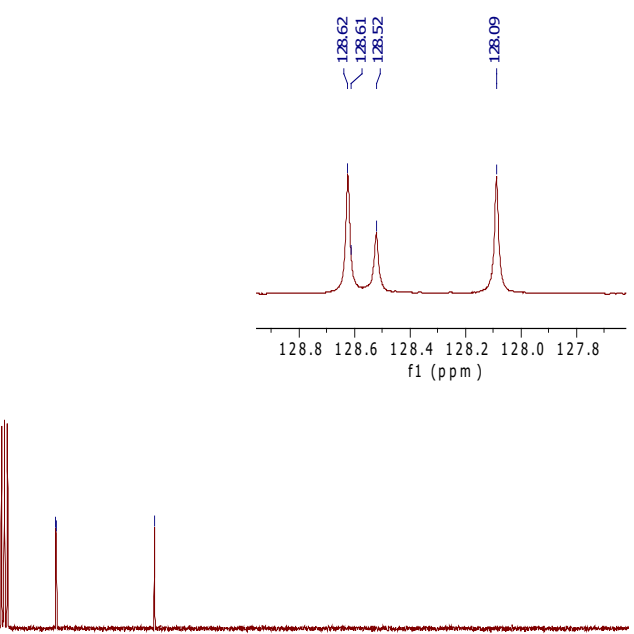

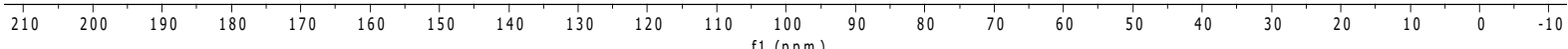




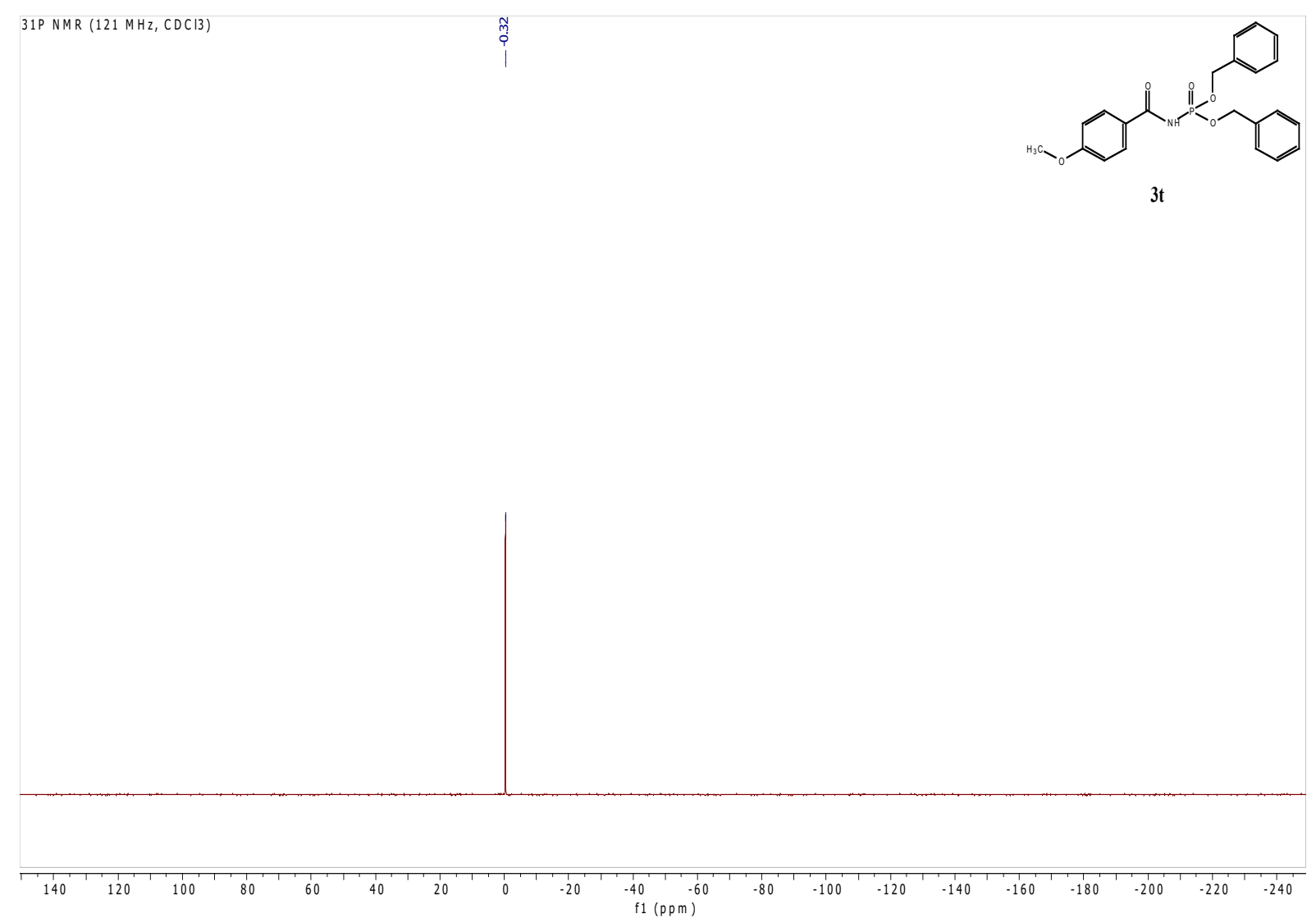

DAD1 A, Sig=254,4 Ref=off (V:108-20IIAINIIWC-1176-015-4.D)

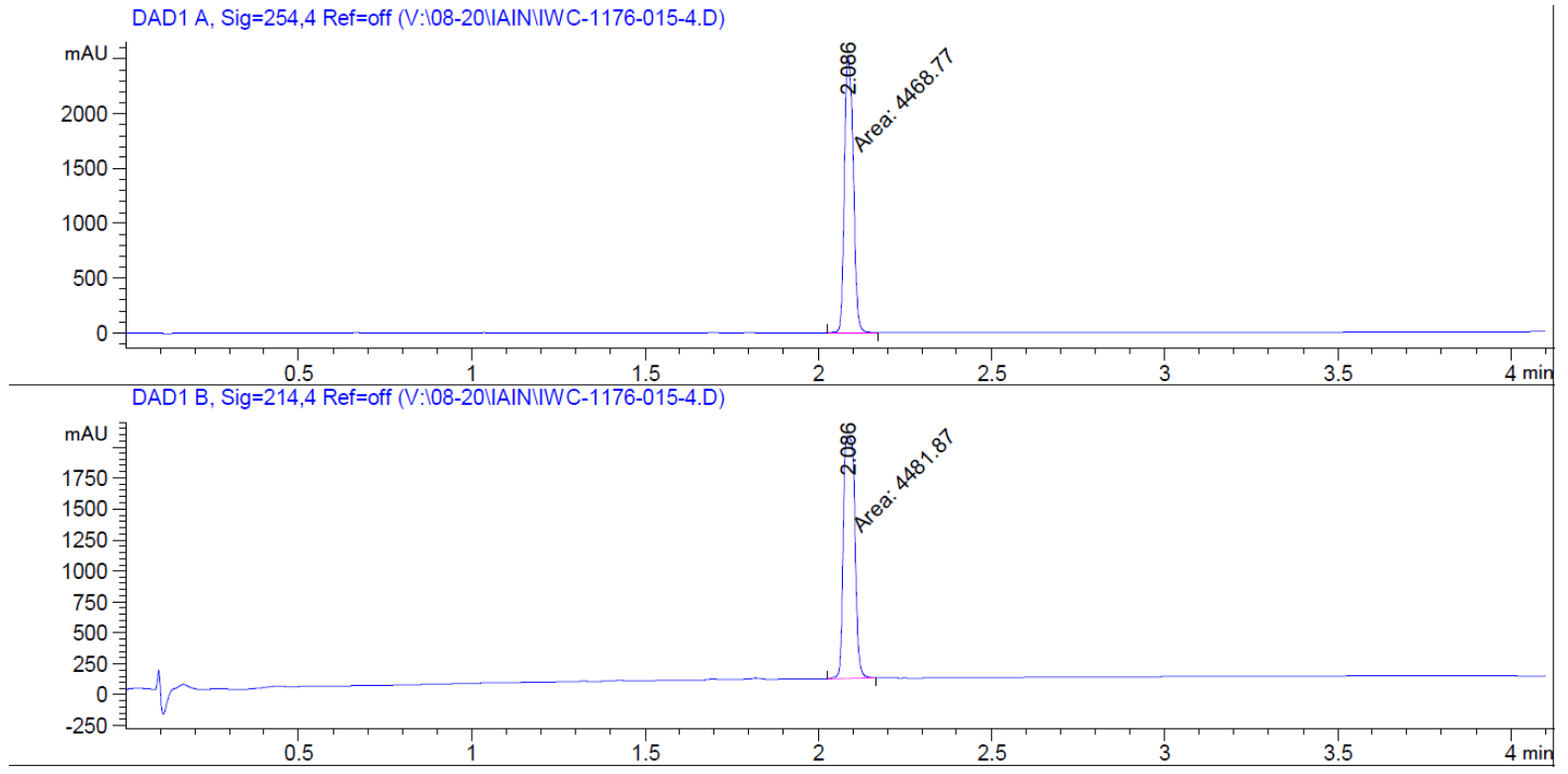


Dibenzyl (2-fluorobenzoyl)phosphoramidate (3u)

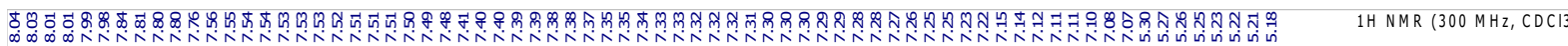
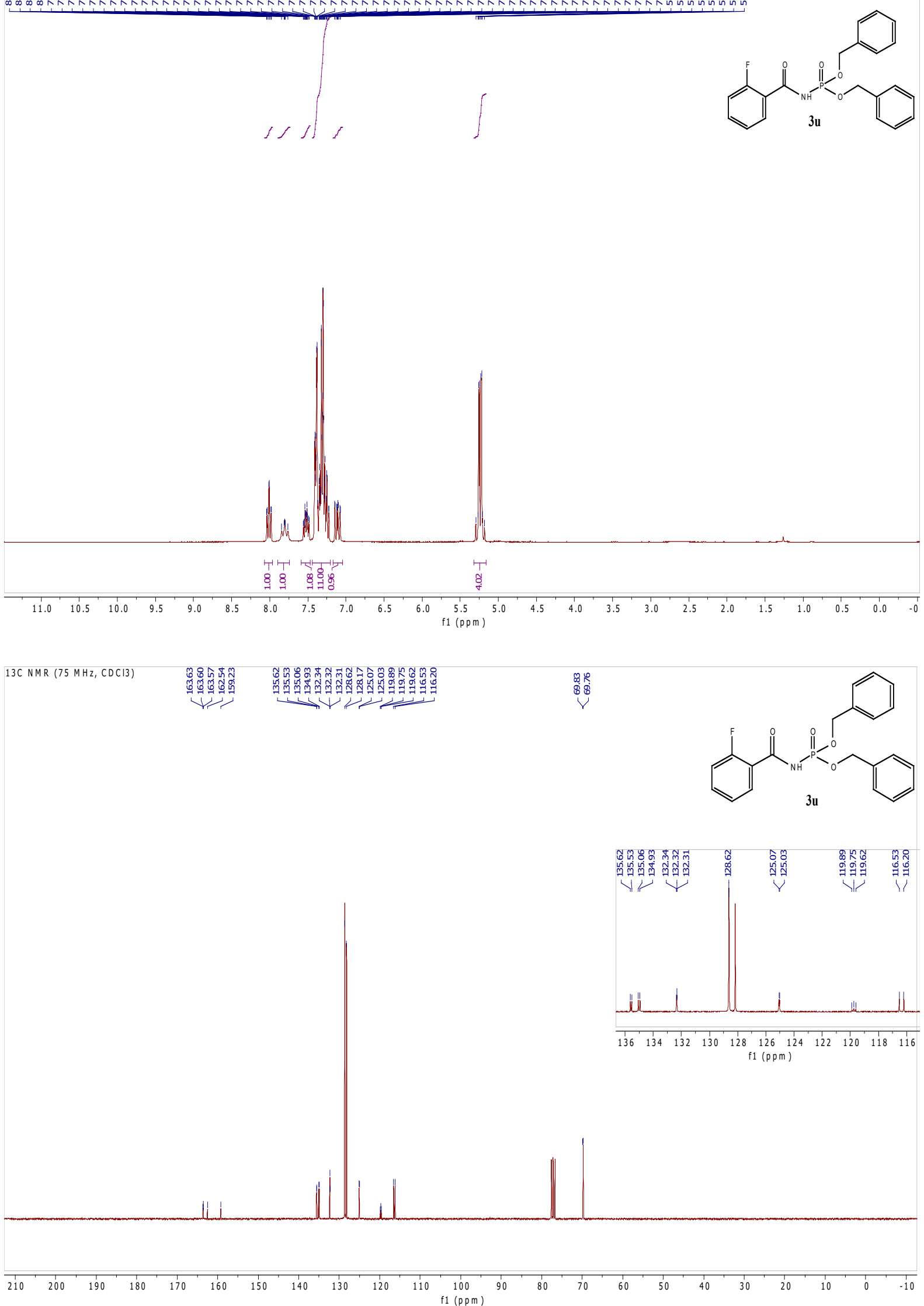

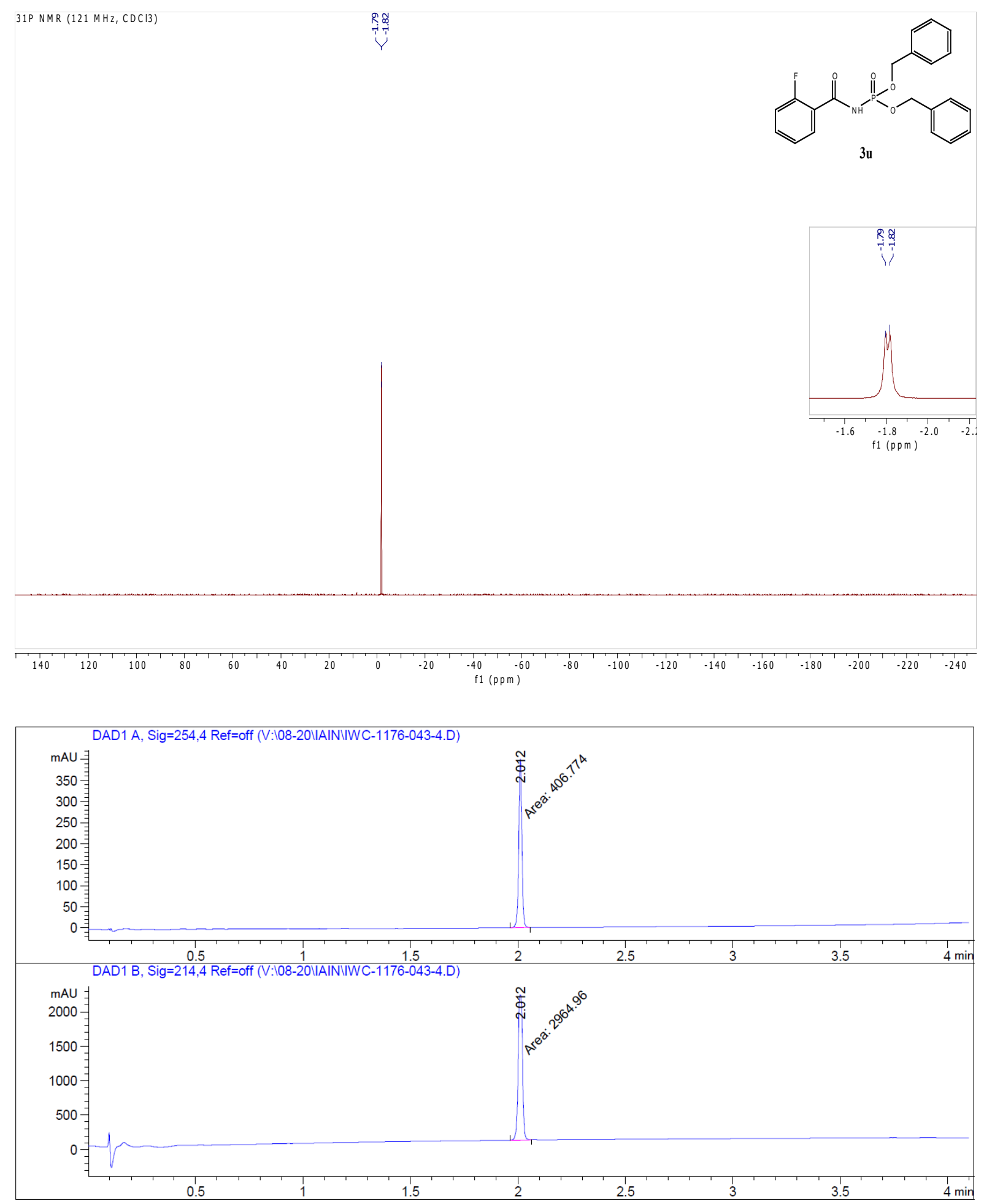
Dibenzyl (2-(methylthio)benzoyl)phosphoramidate (3v)
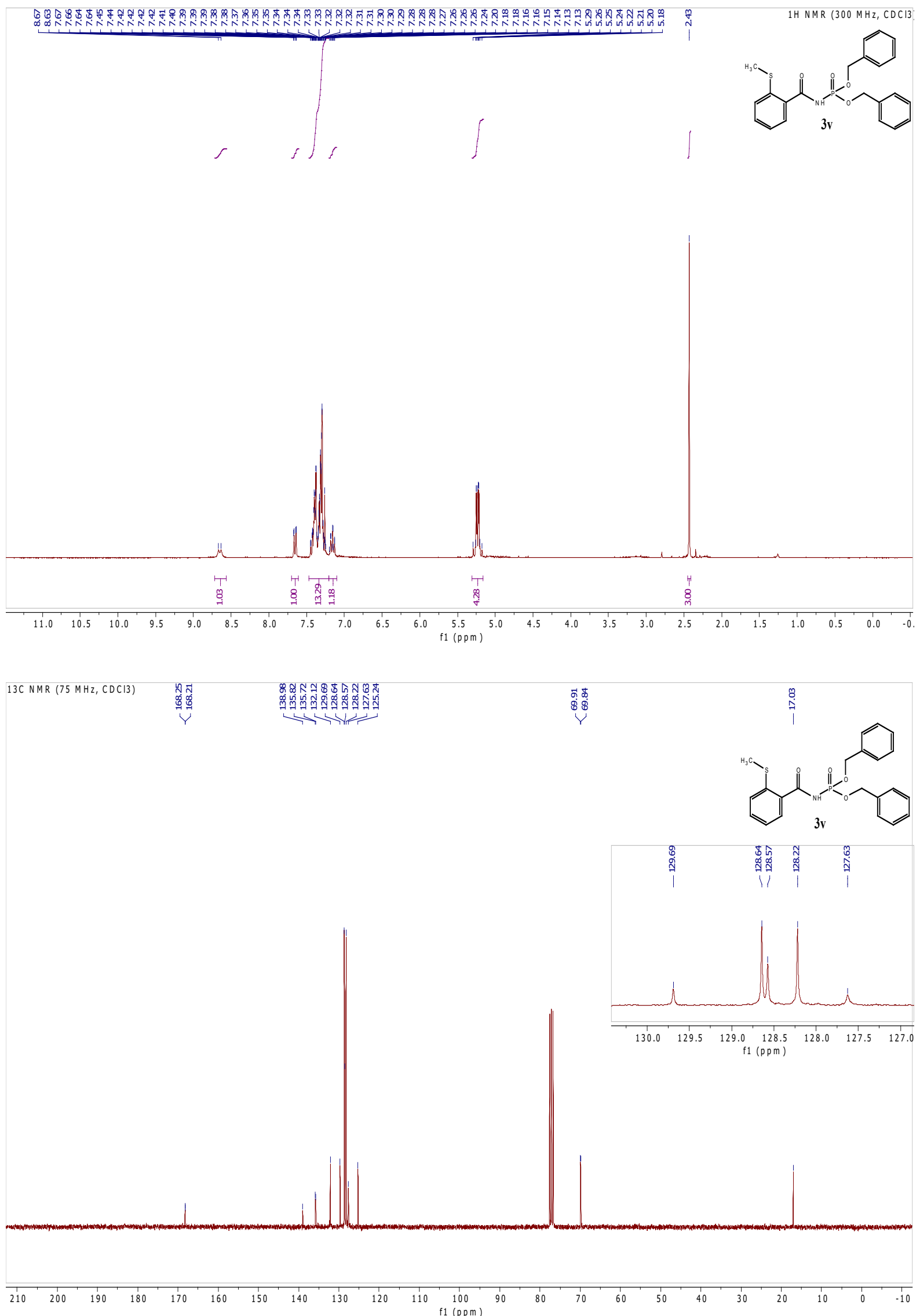


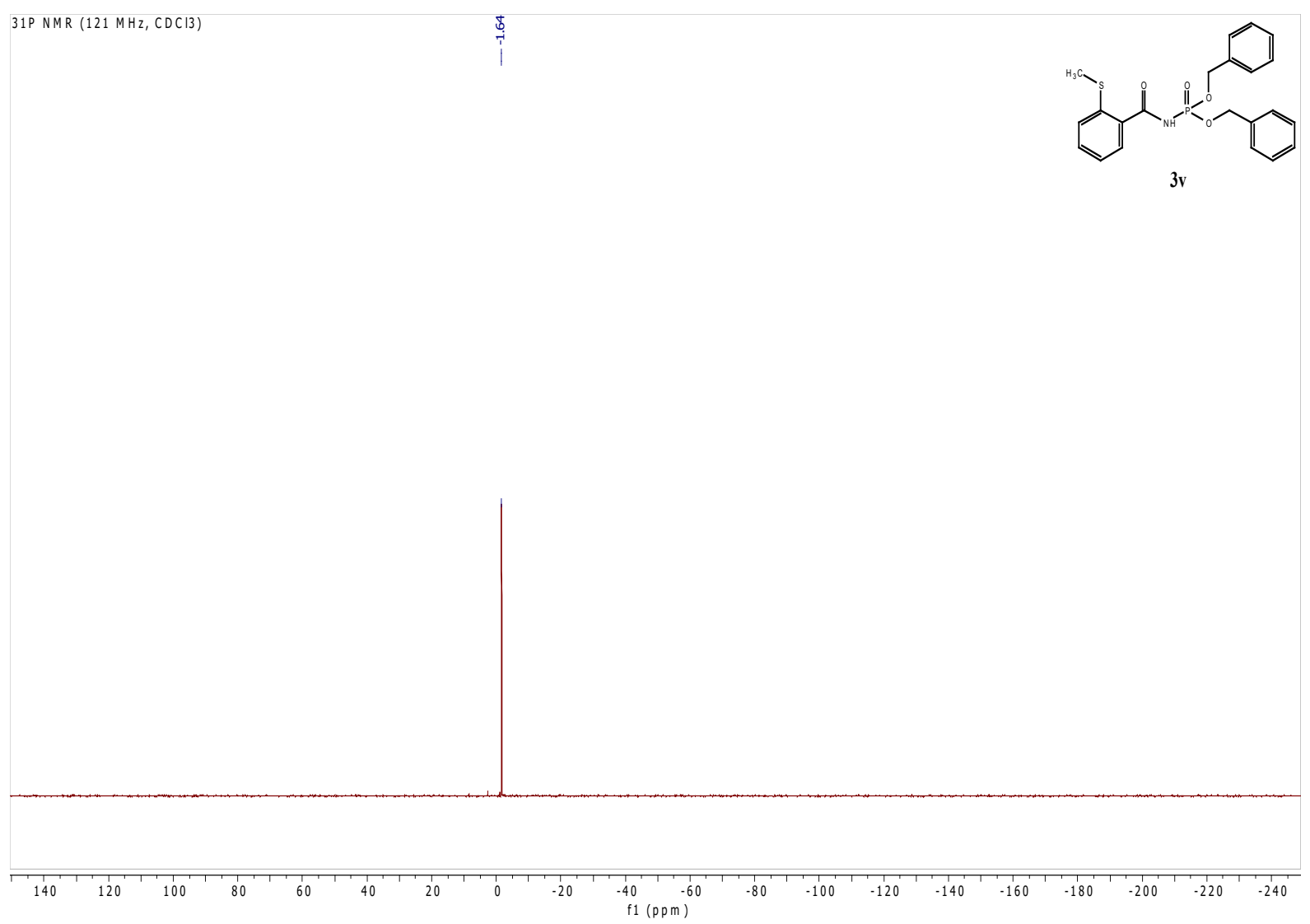


Dibenzyl (2-nitrobenzoyl)phosphoramidate (3w)

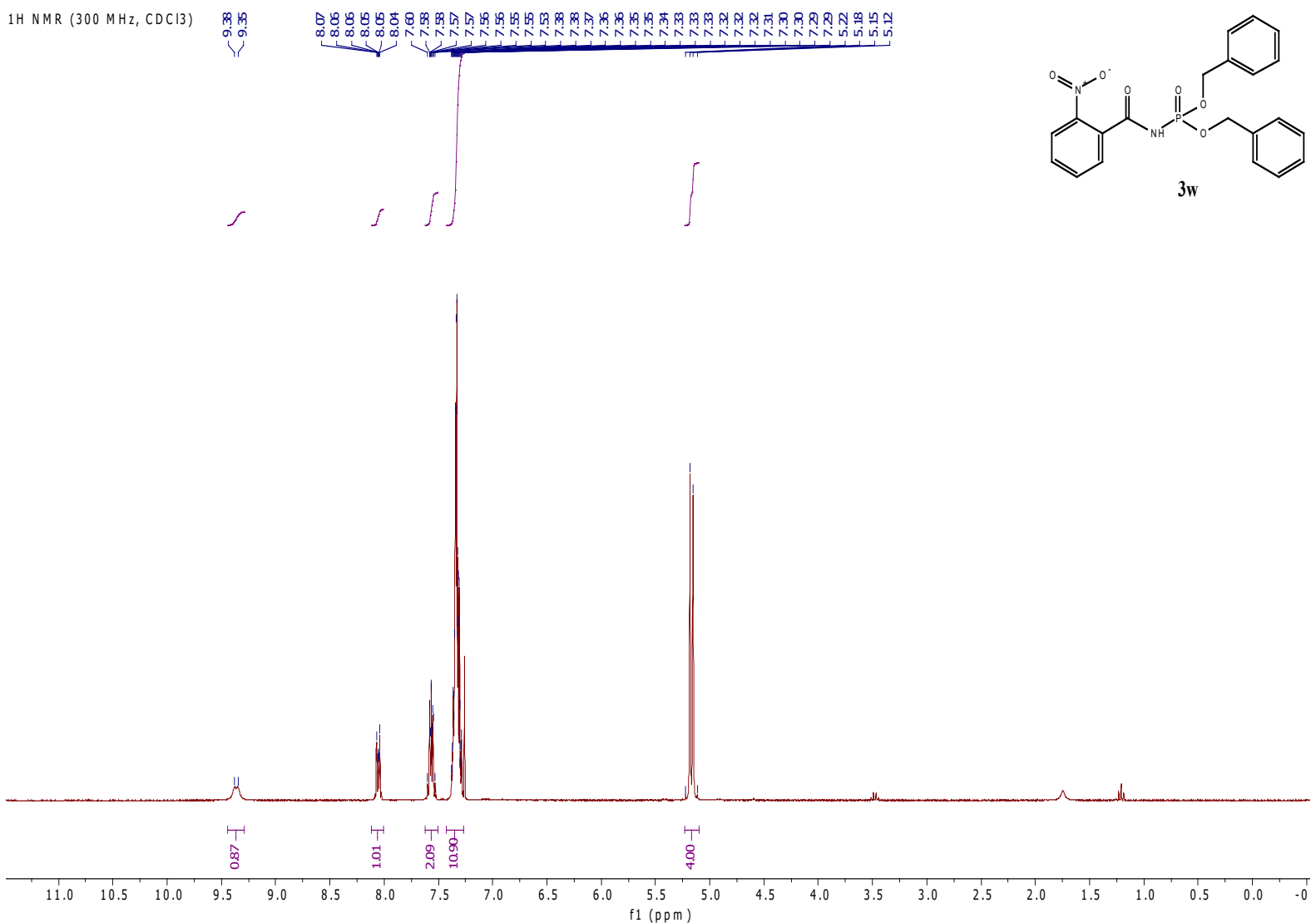

13C NMR (75 MHz, CDCl3) 过

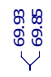
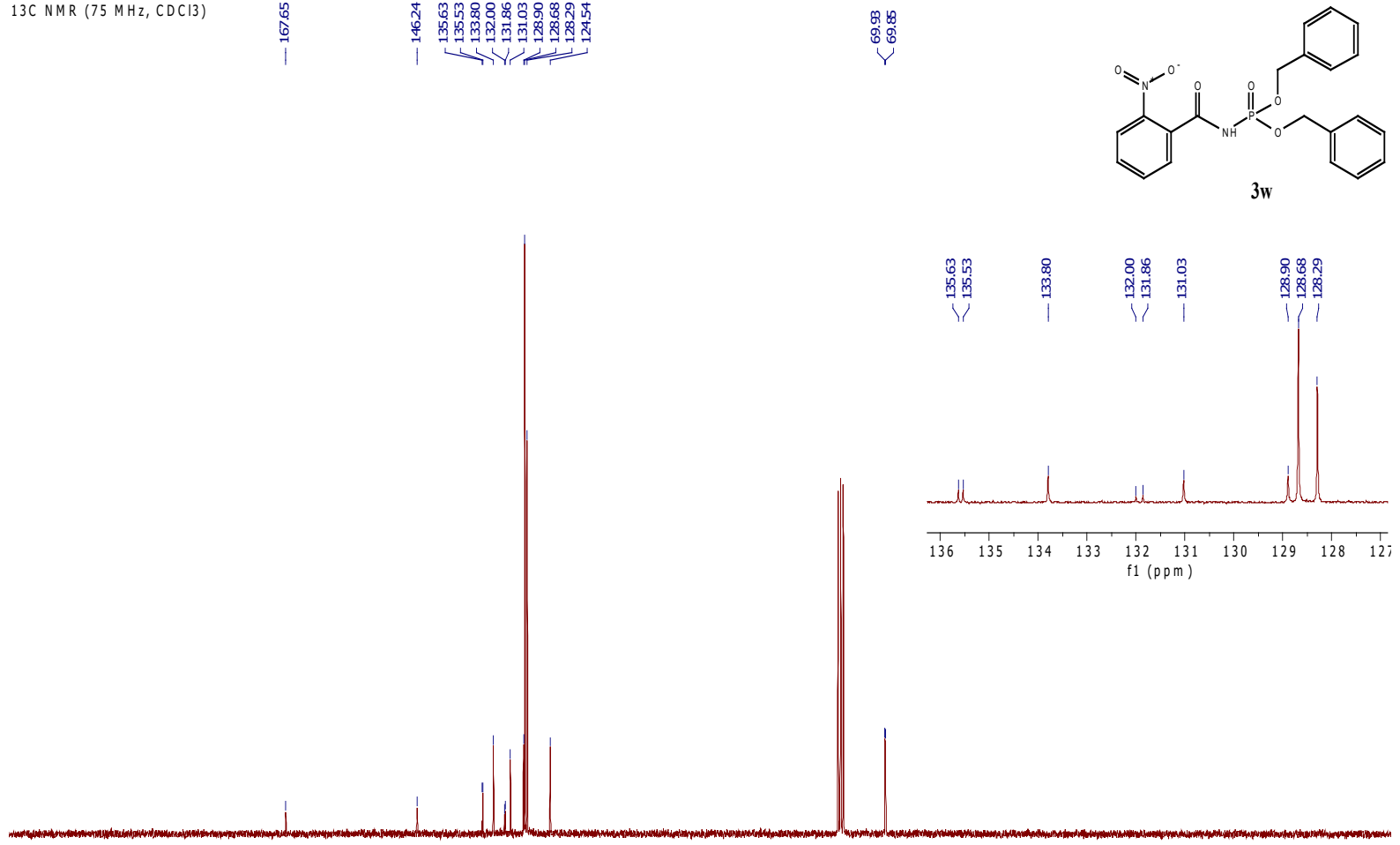

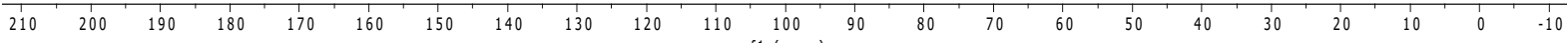



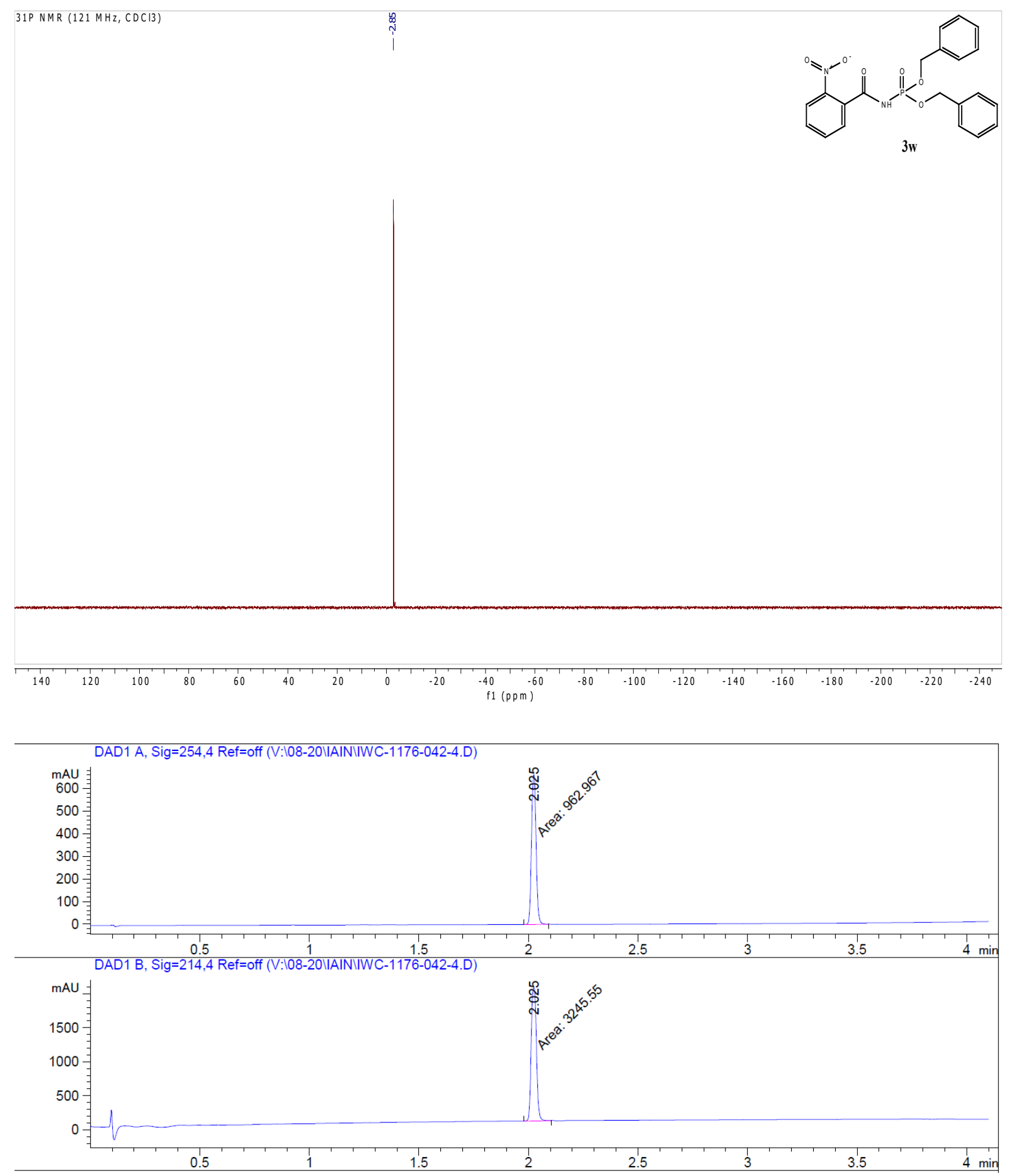
Dibenzyl (4-cyanobenzoyl)phosphoramidate (3x)
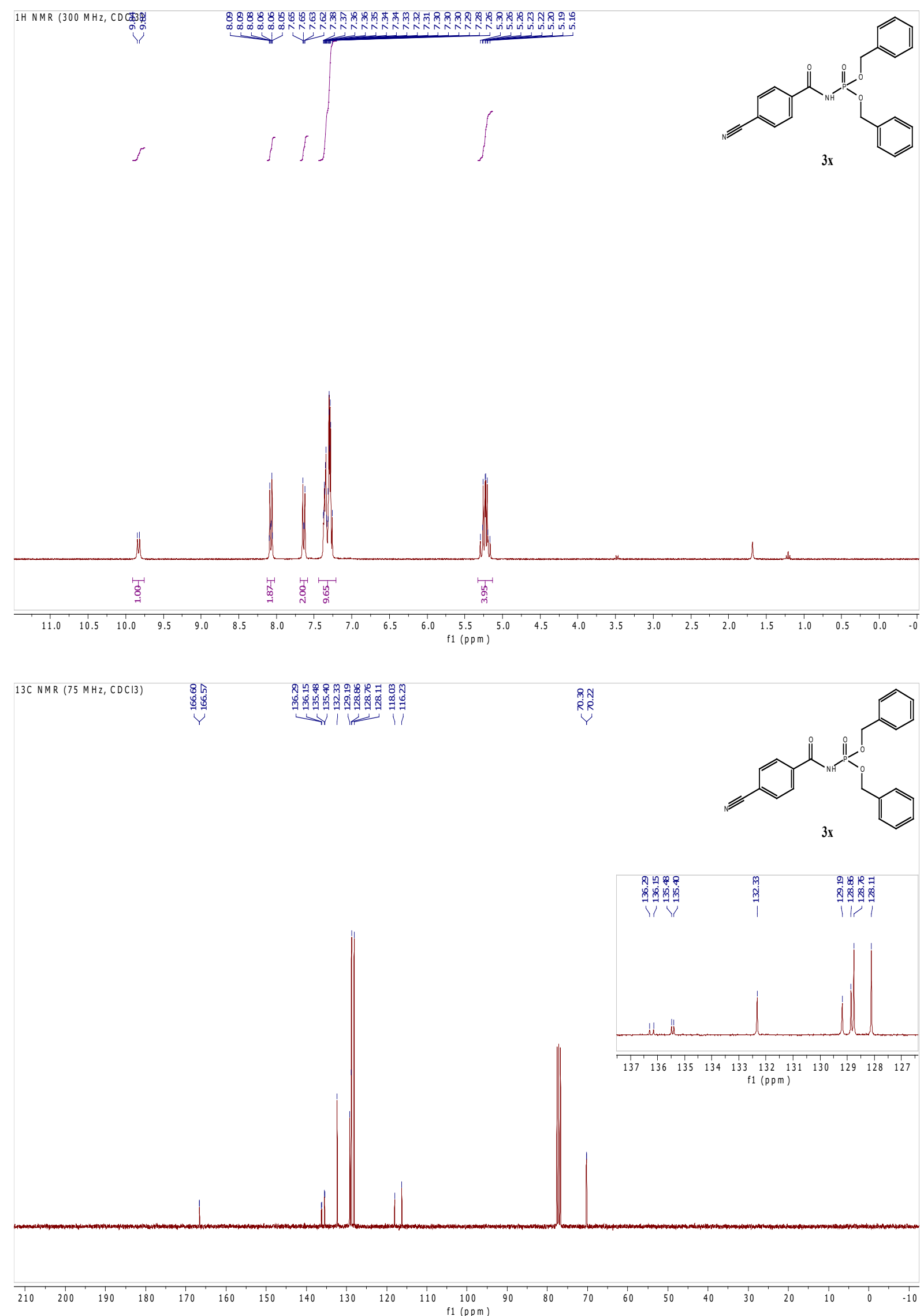


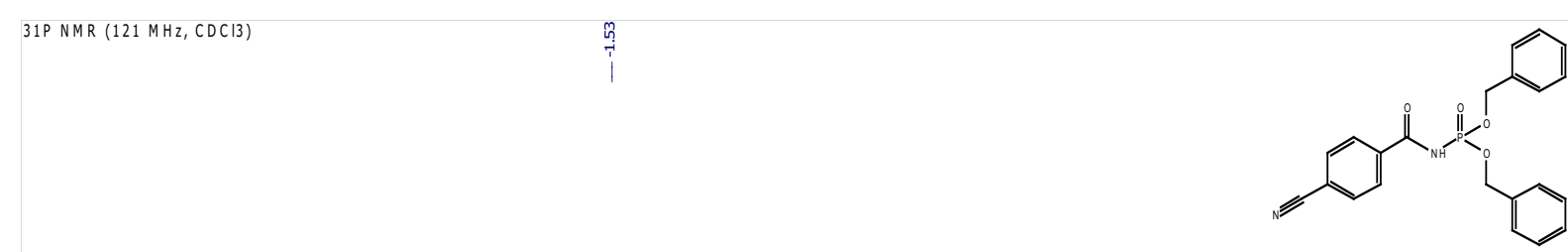

$3 x$
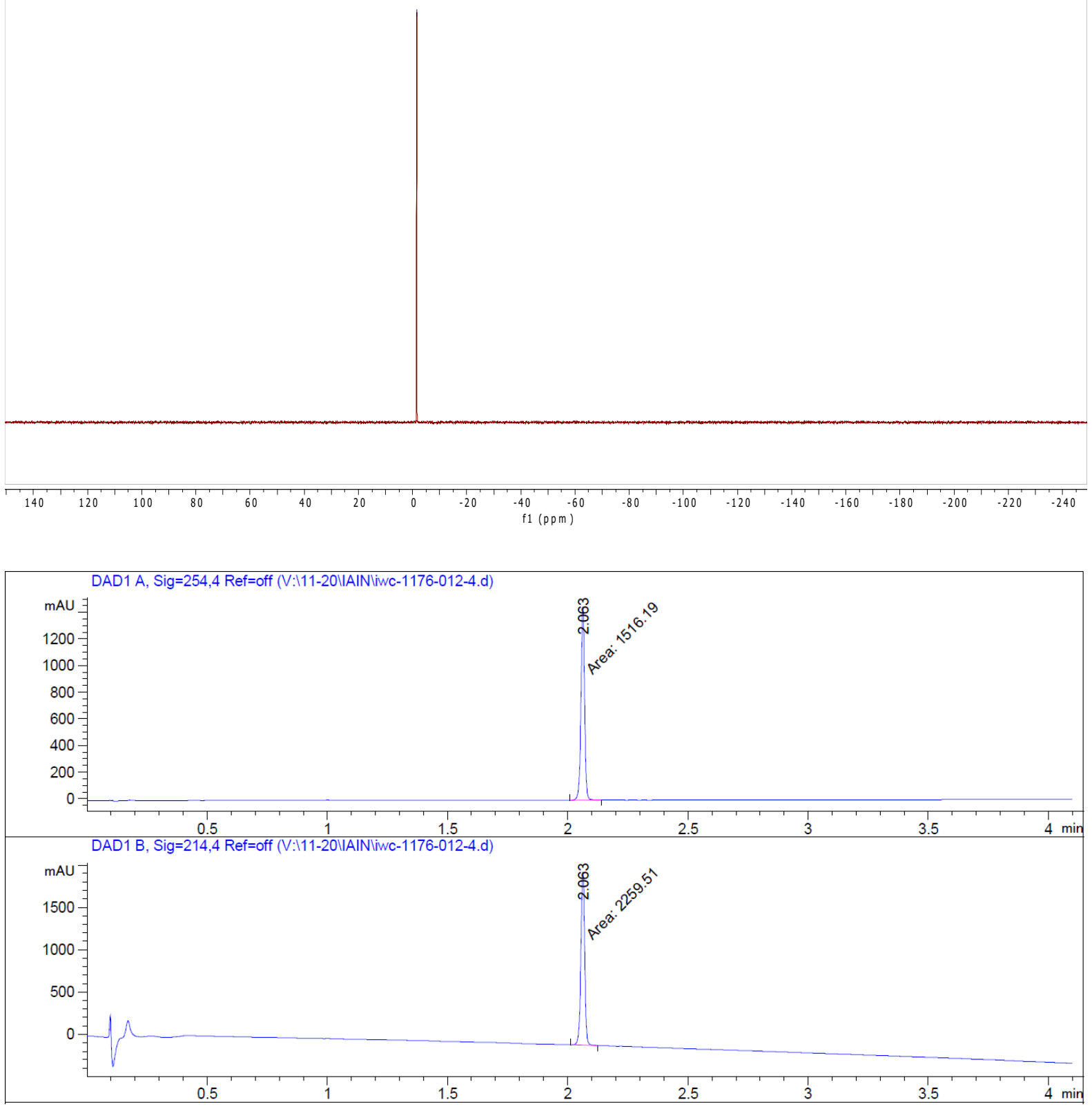
Dibenzyl (4-ethynylbenzoyl)phosphoramidate (3y)
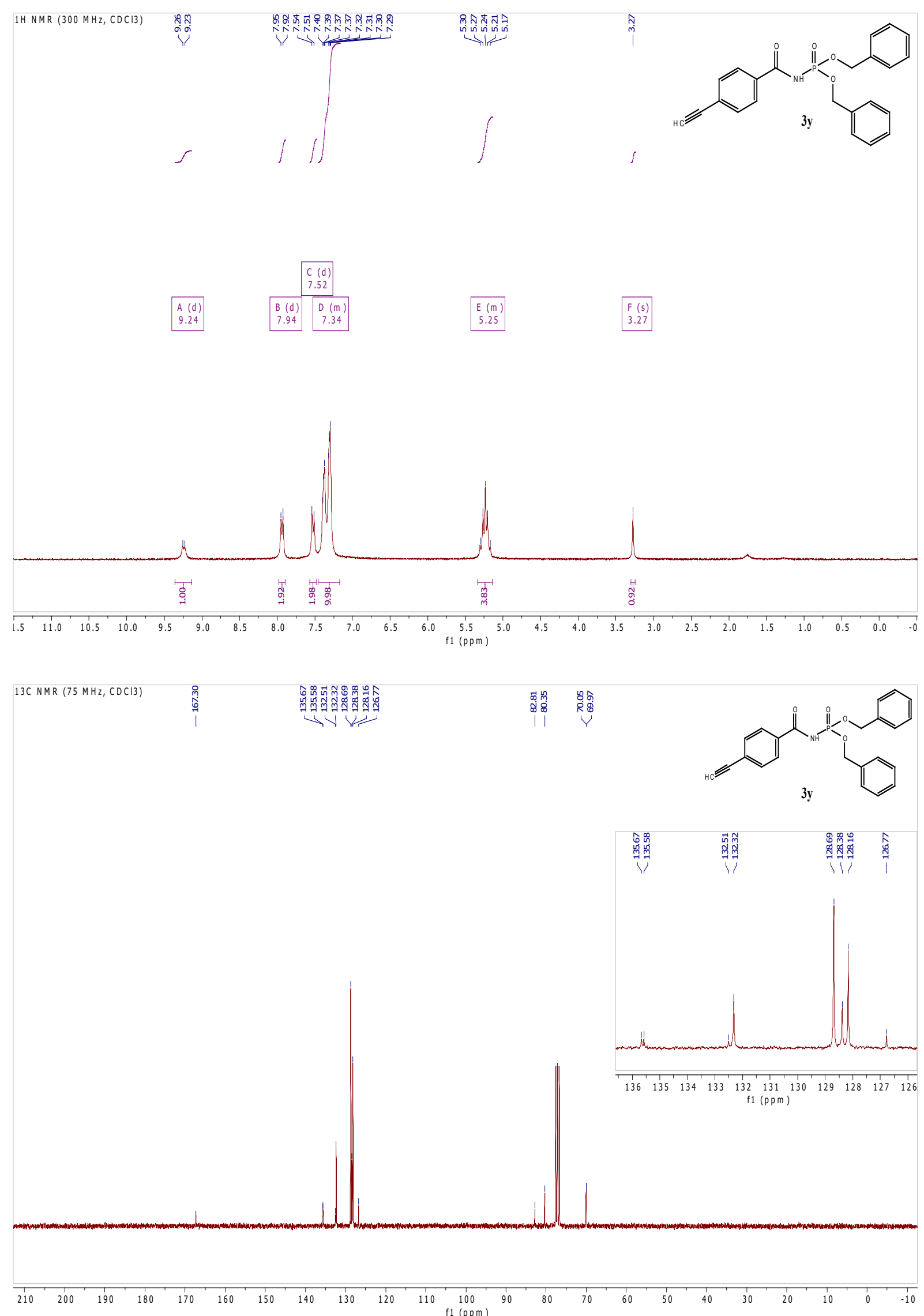

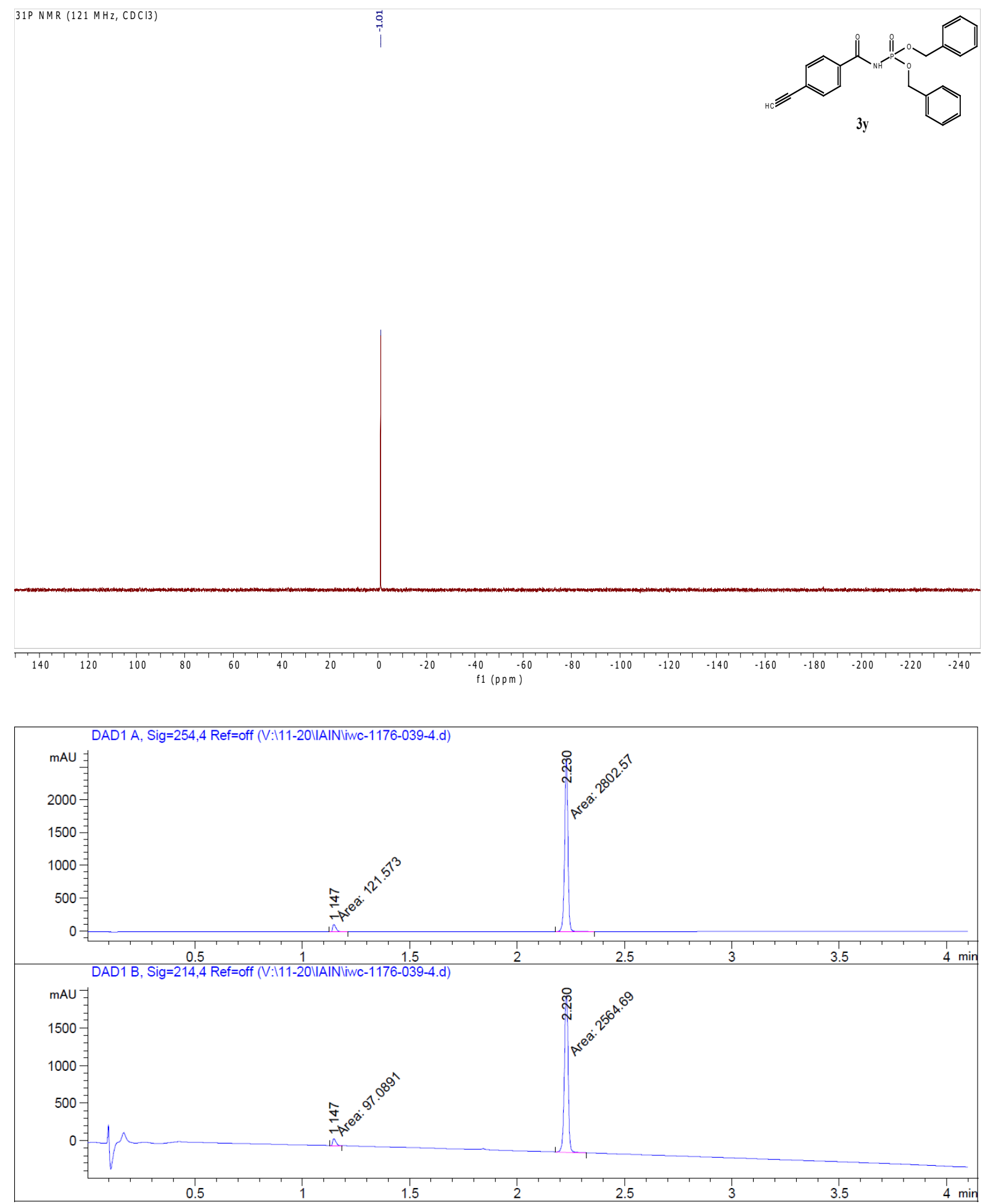
Dibenzyl (4-carbamoylbenzoyl)phosphoramidate (3z)
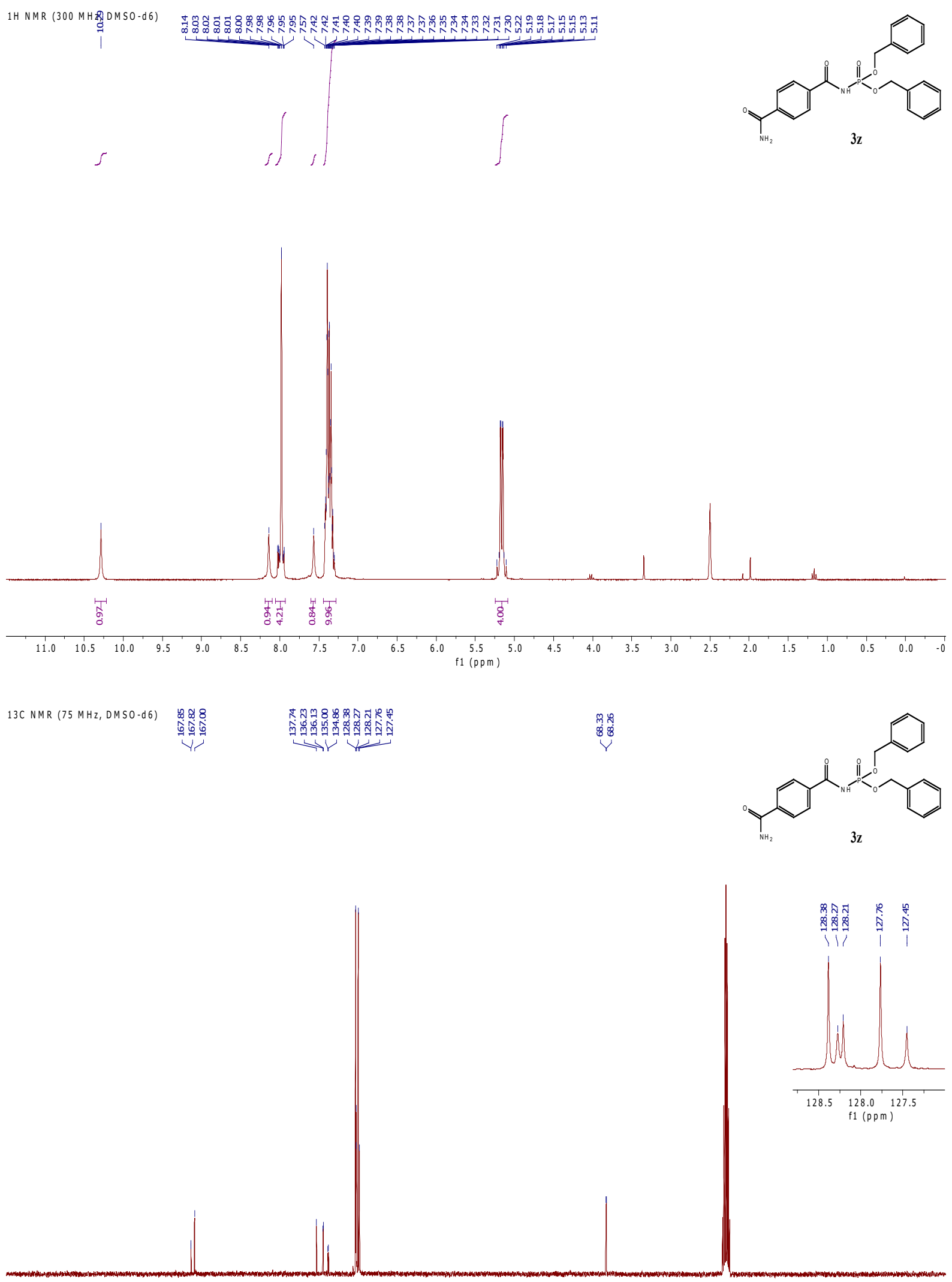

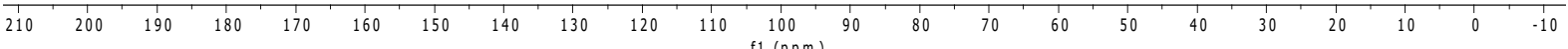


31P NMR (121 MHz, DMSO-d6)

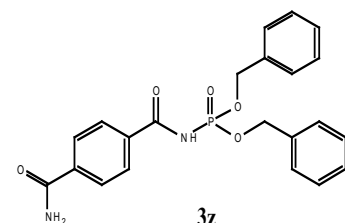

$3 z$

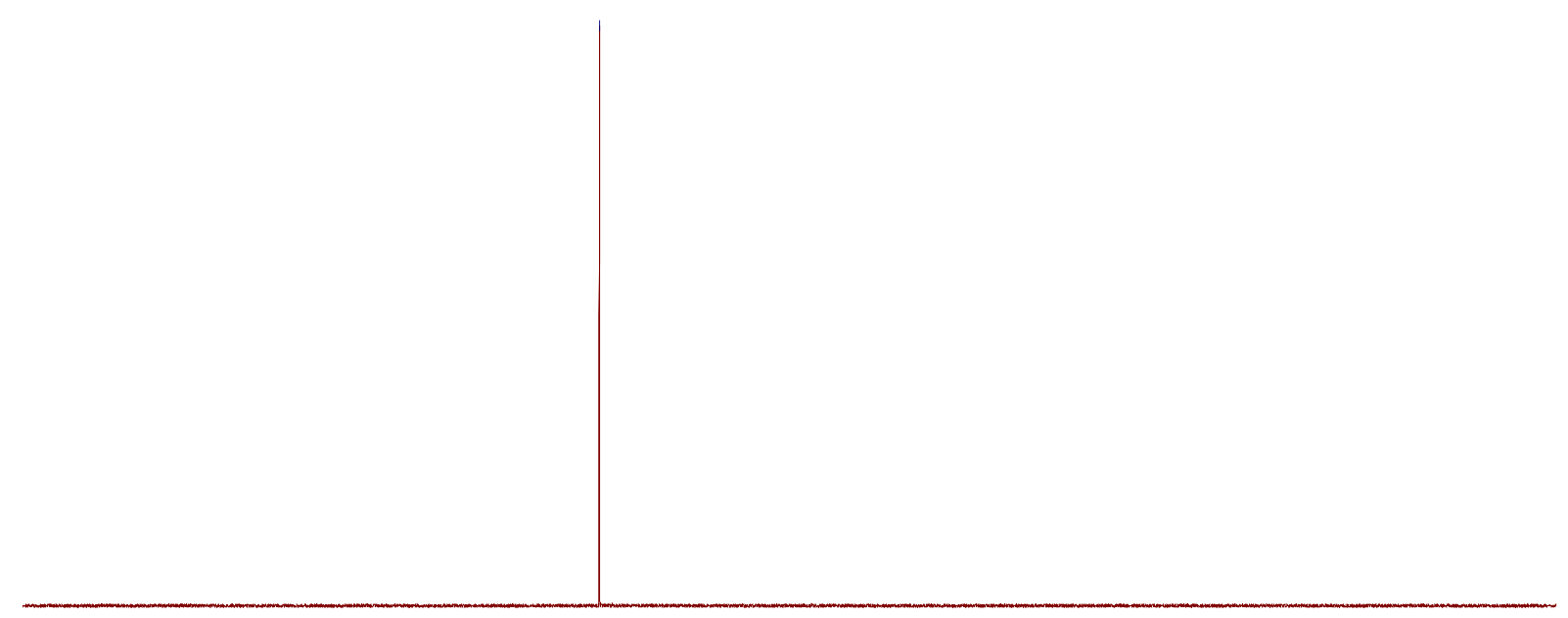

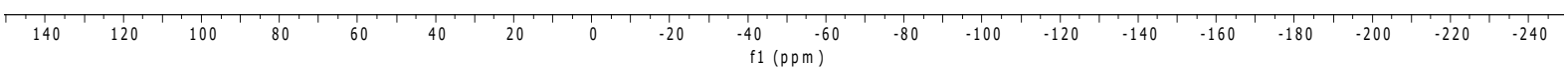

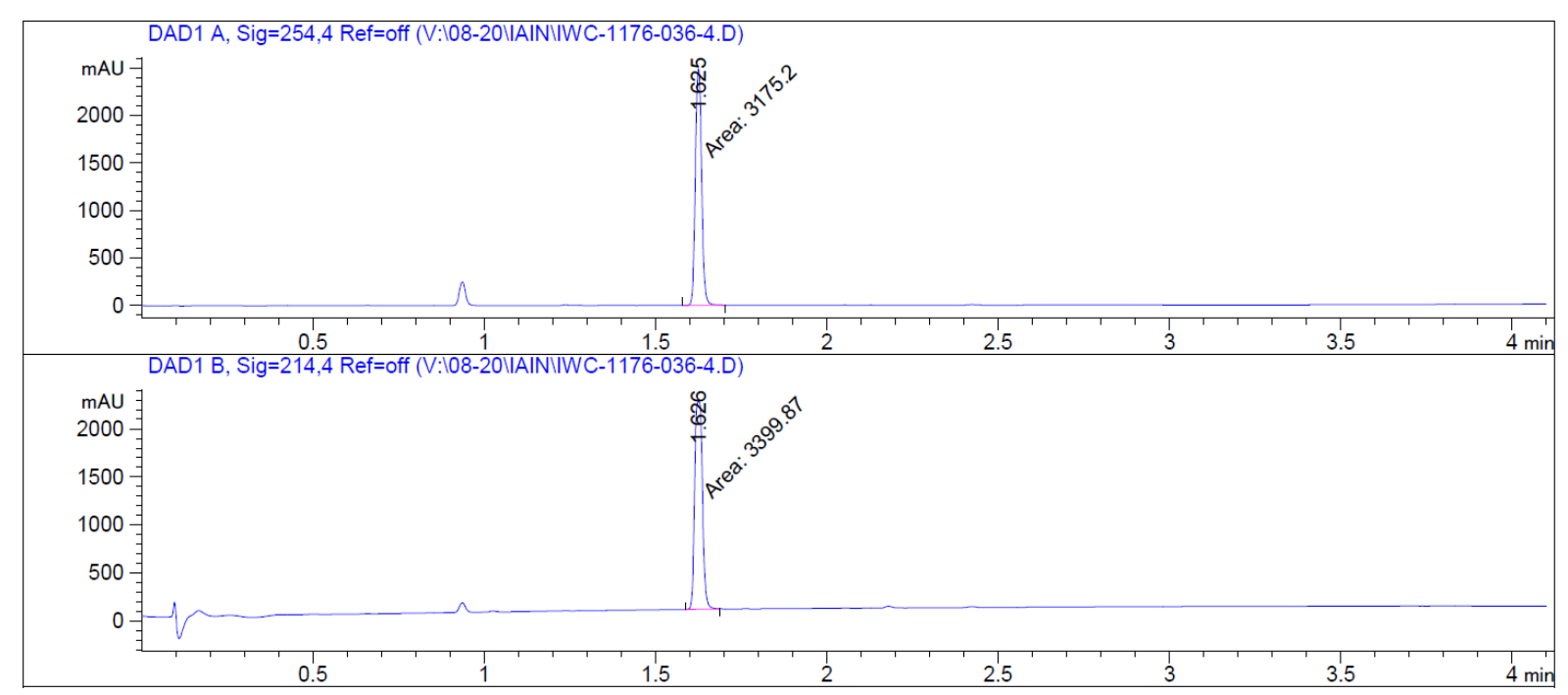


tert-Butyl ((2S,3R)-1-((bis(benzyloxy)phosphoryl)amino)-3-hydroxy-1-oxobutan-2yl)carbamate (3aa)

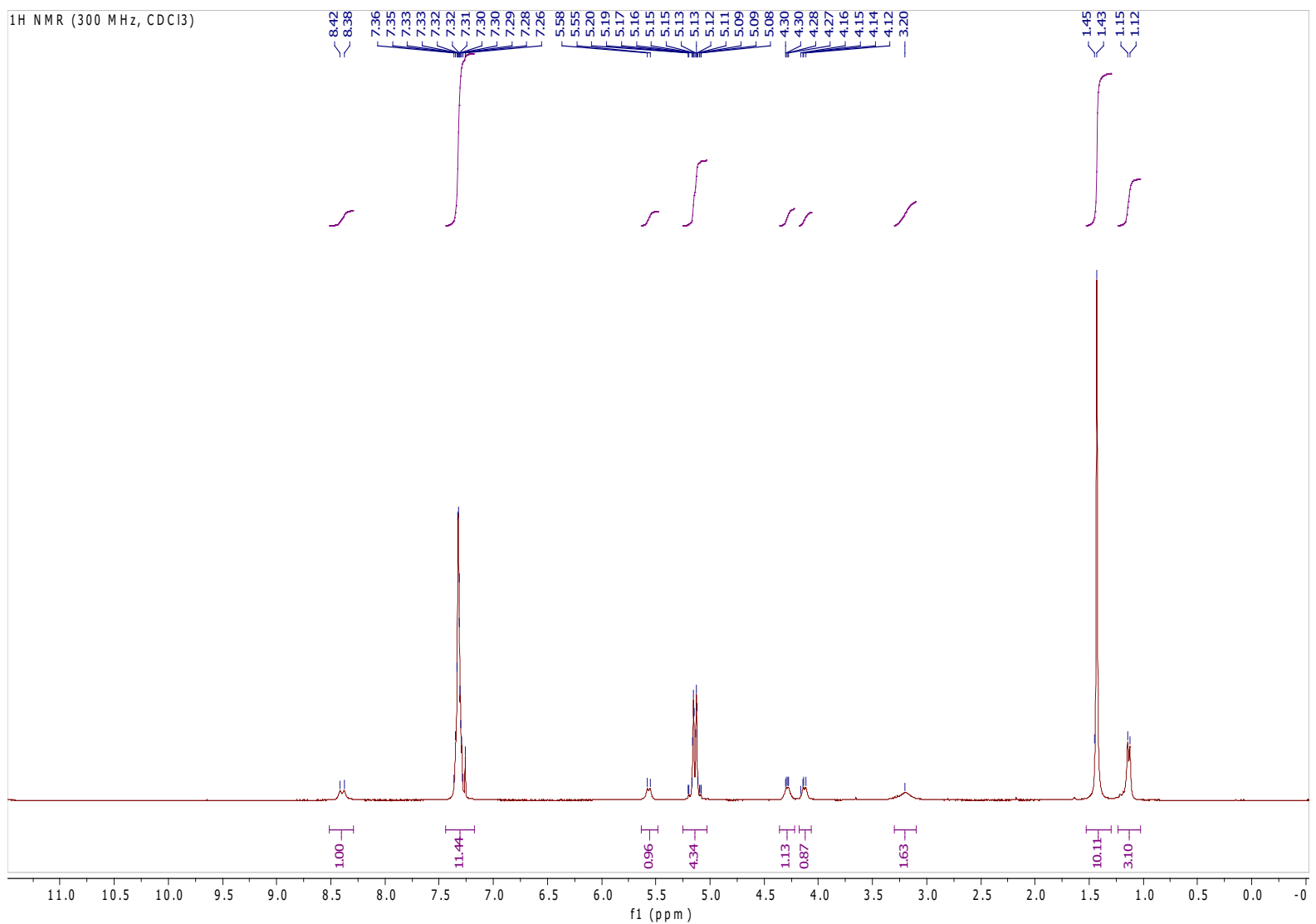



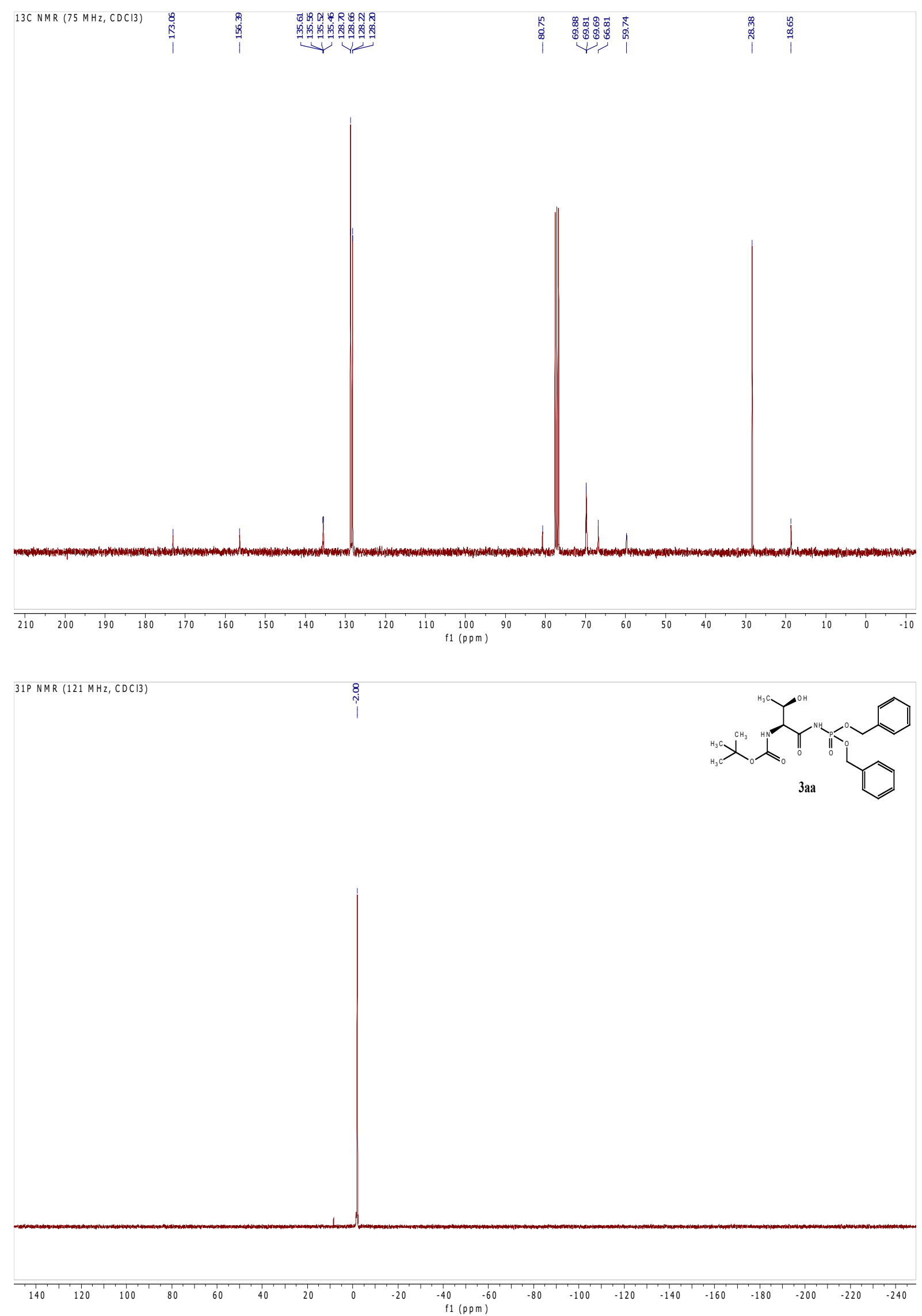


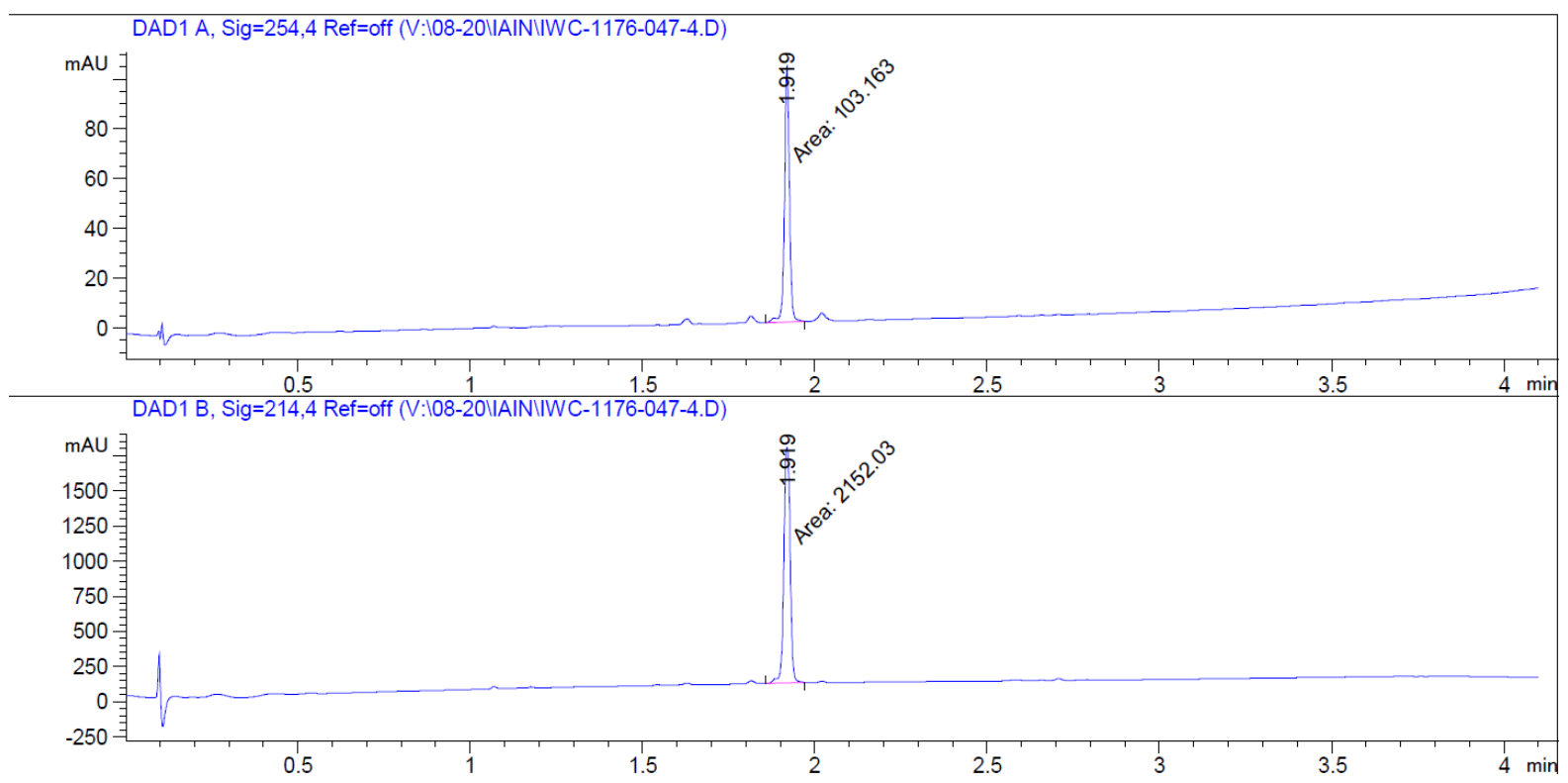


(S)-tert-Butyl (1-((bis(benzyloxy)phosphoryl)amino)-1-oxo-3-phenylpropan-2yl)carbamate (3ab)

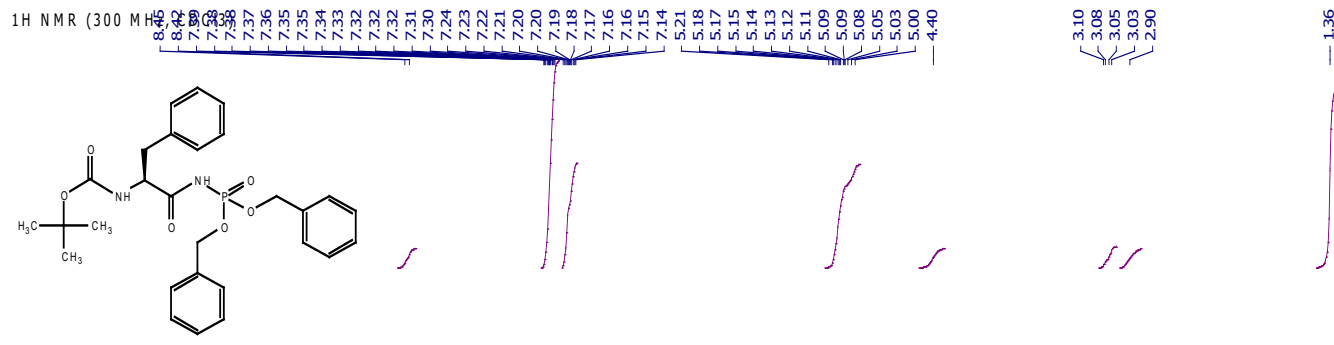

3ab

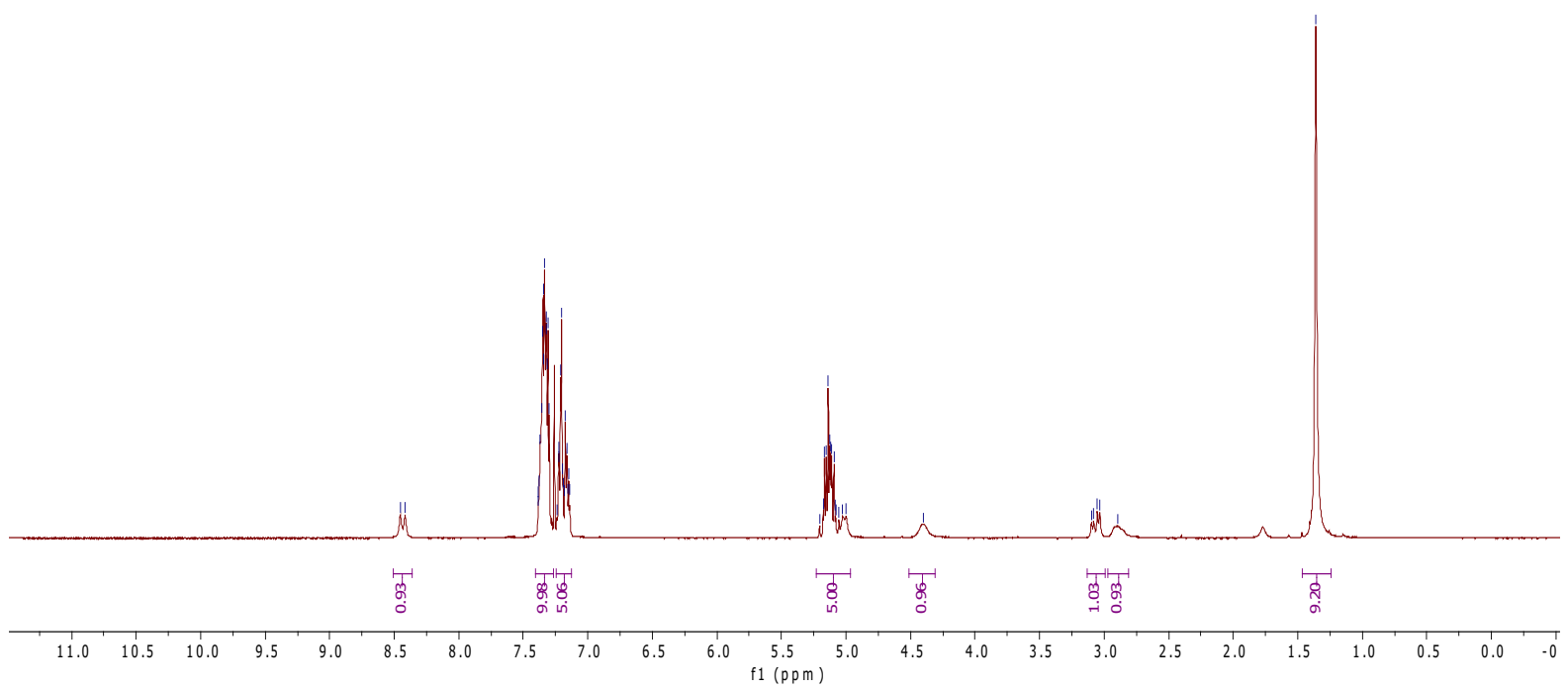




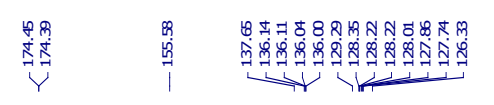

幽

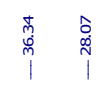
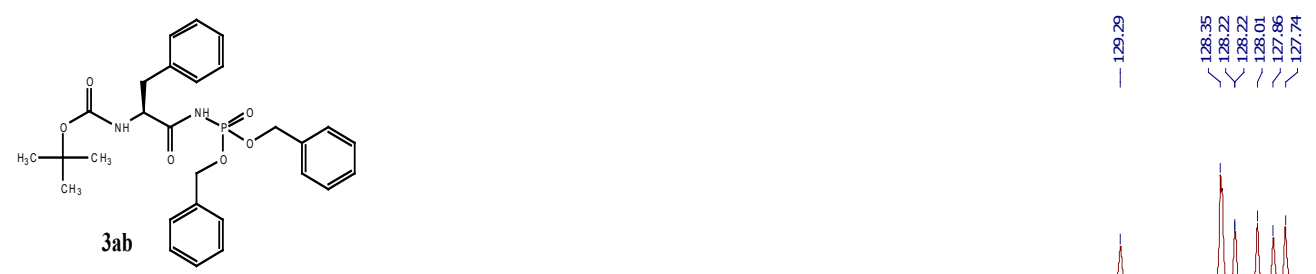

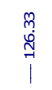
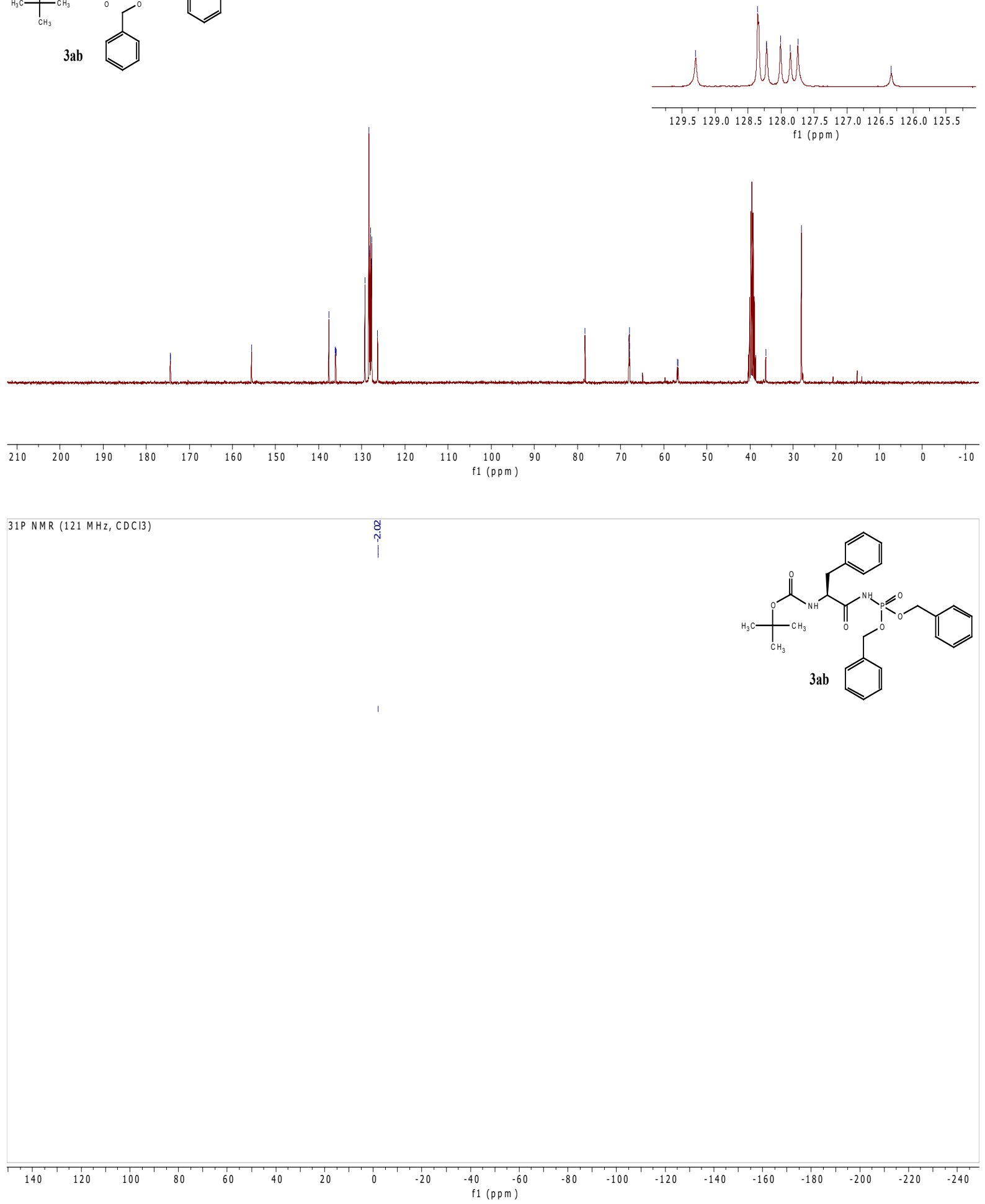

S96 


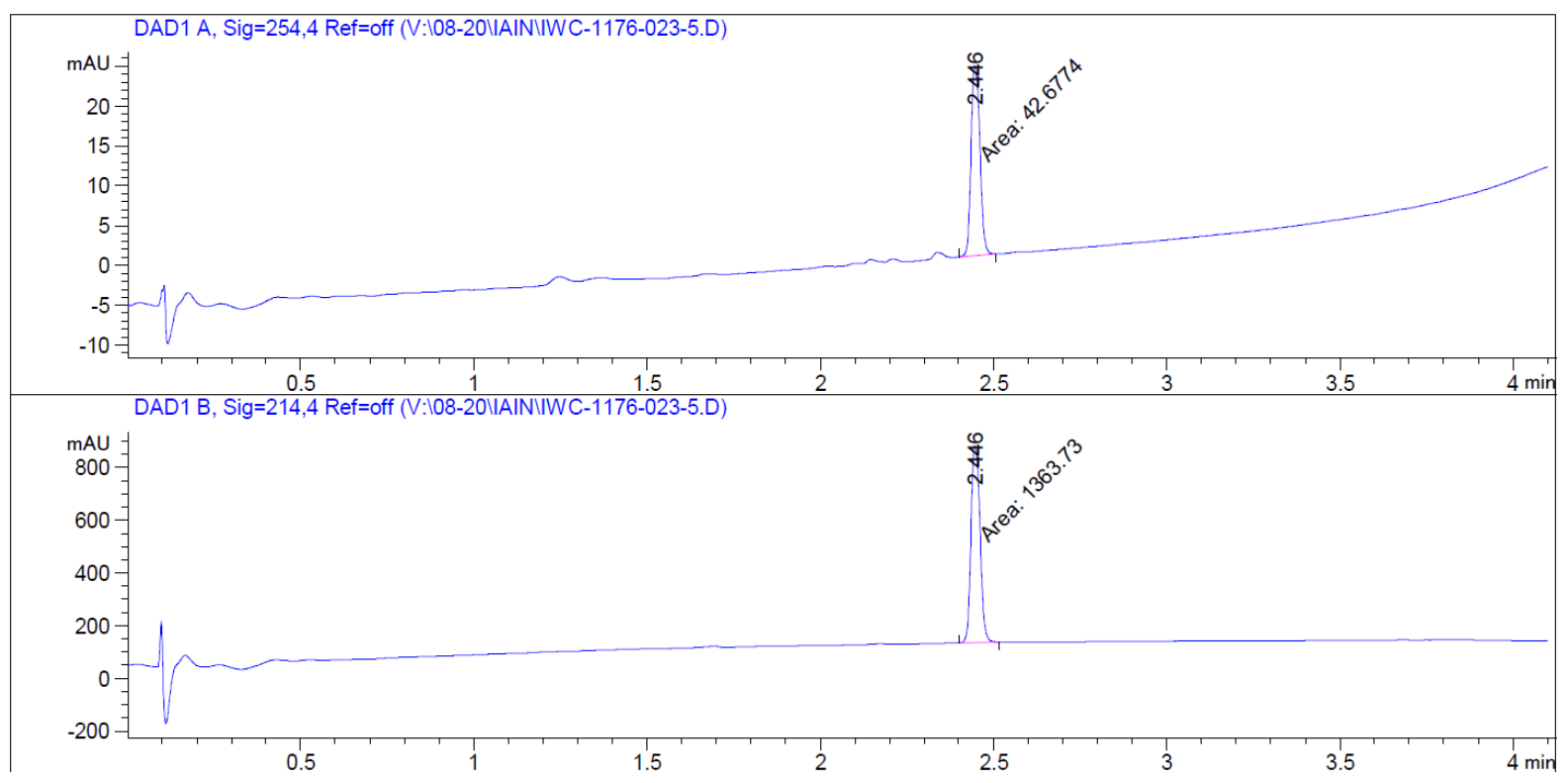


(S)-tert-Butyl (1-((bis(benzyloxy)phosphoryl)amino)-3-(1H-indol-3-yl)-1-oxopropan-2yl)carbamate (3ac)

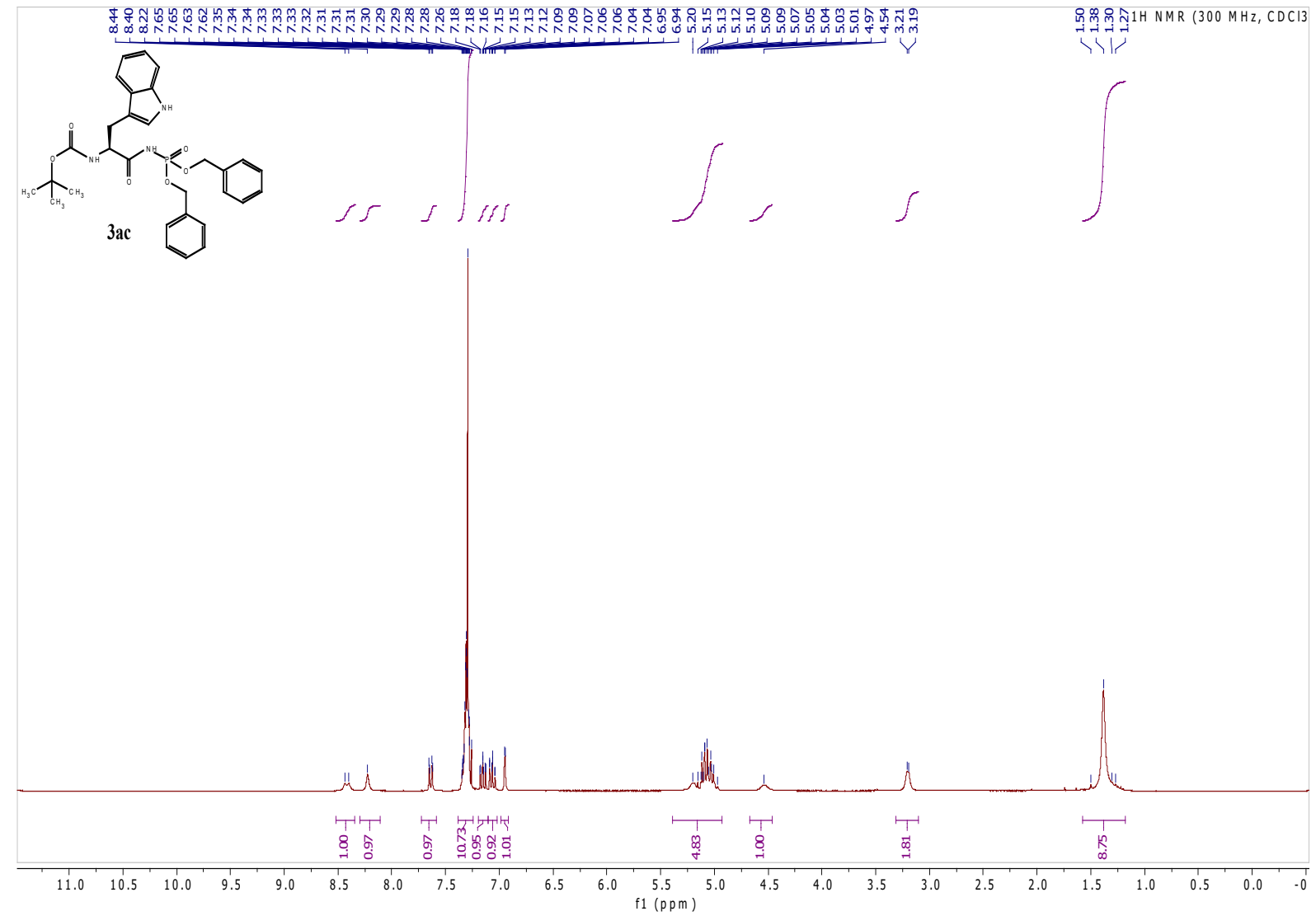




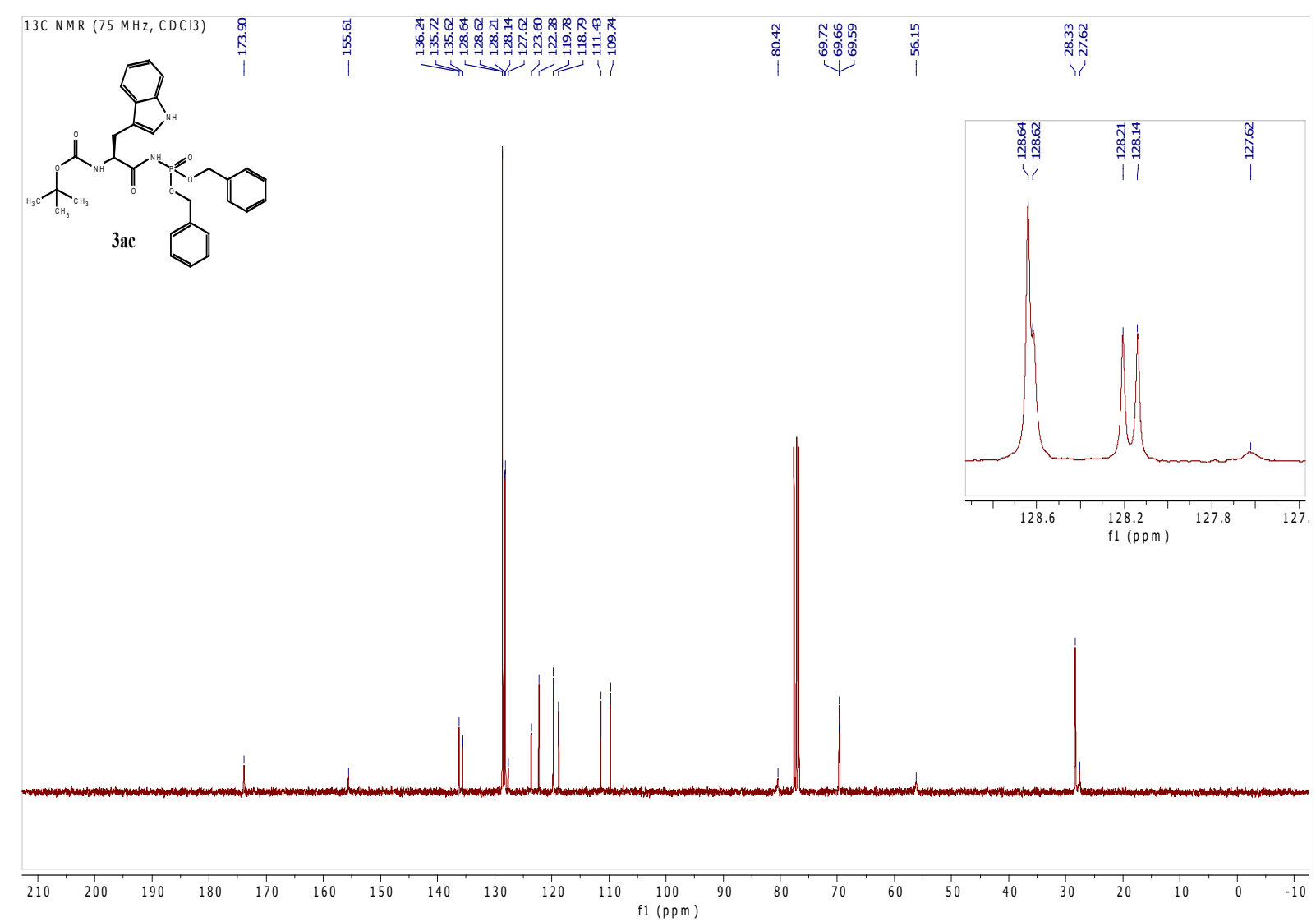

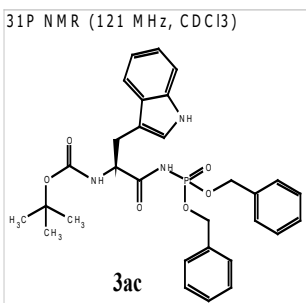




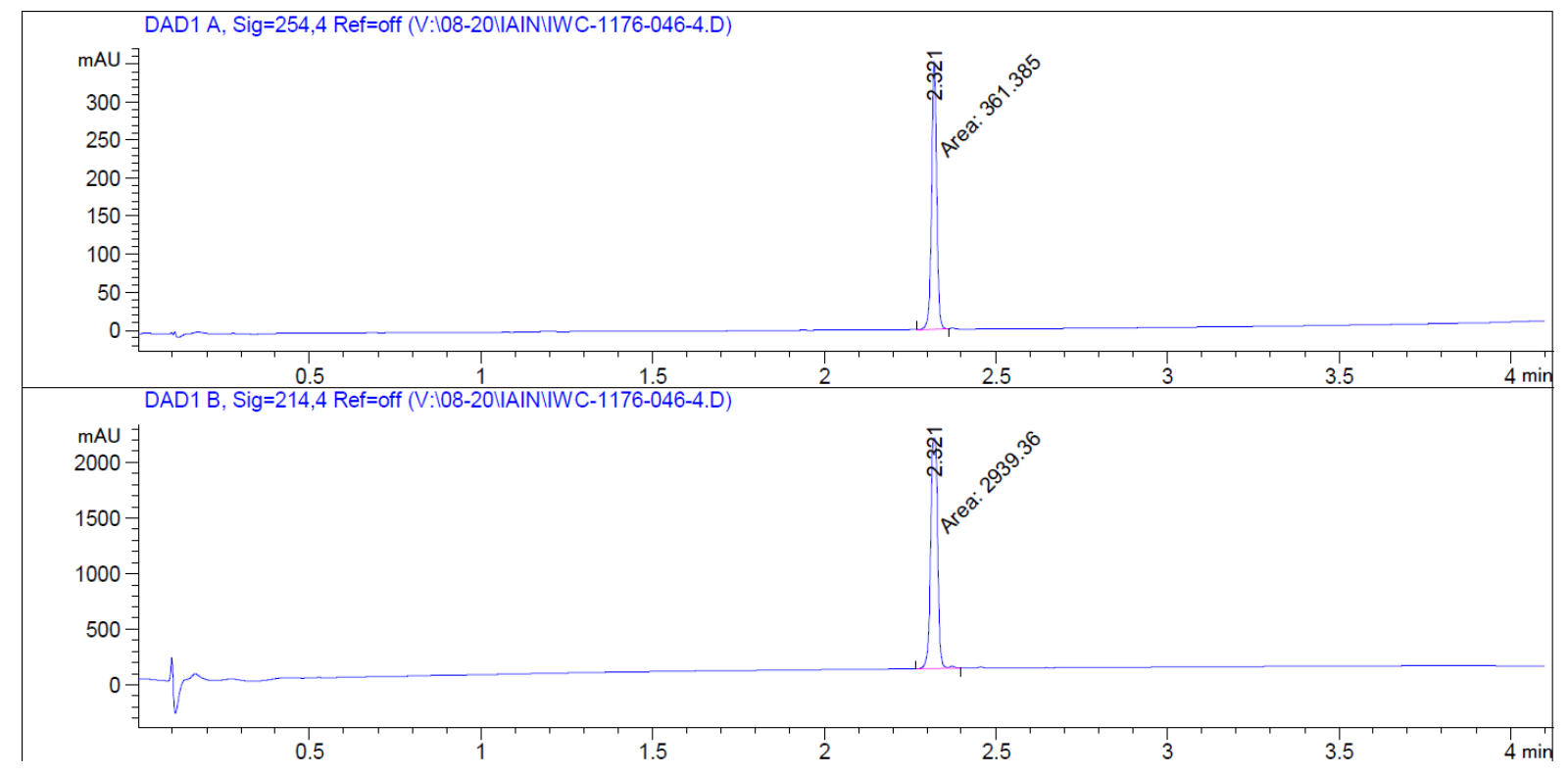


Dibenzyl tridecanoylphosphoramidate (3ad)

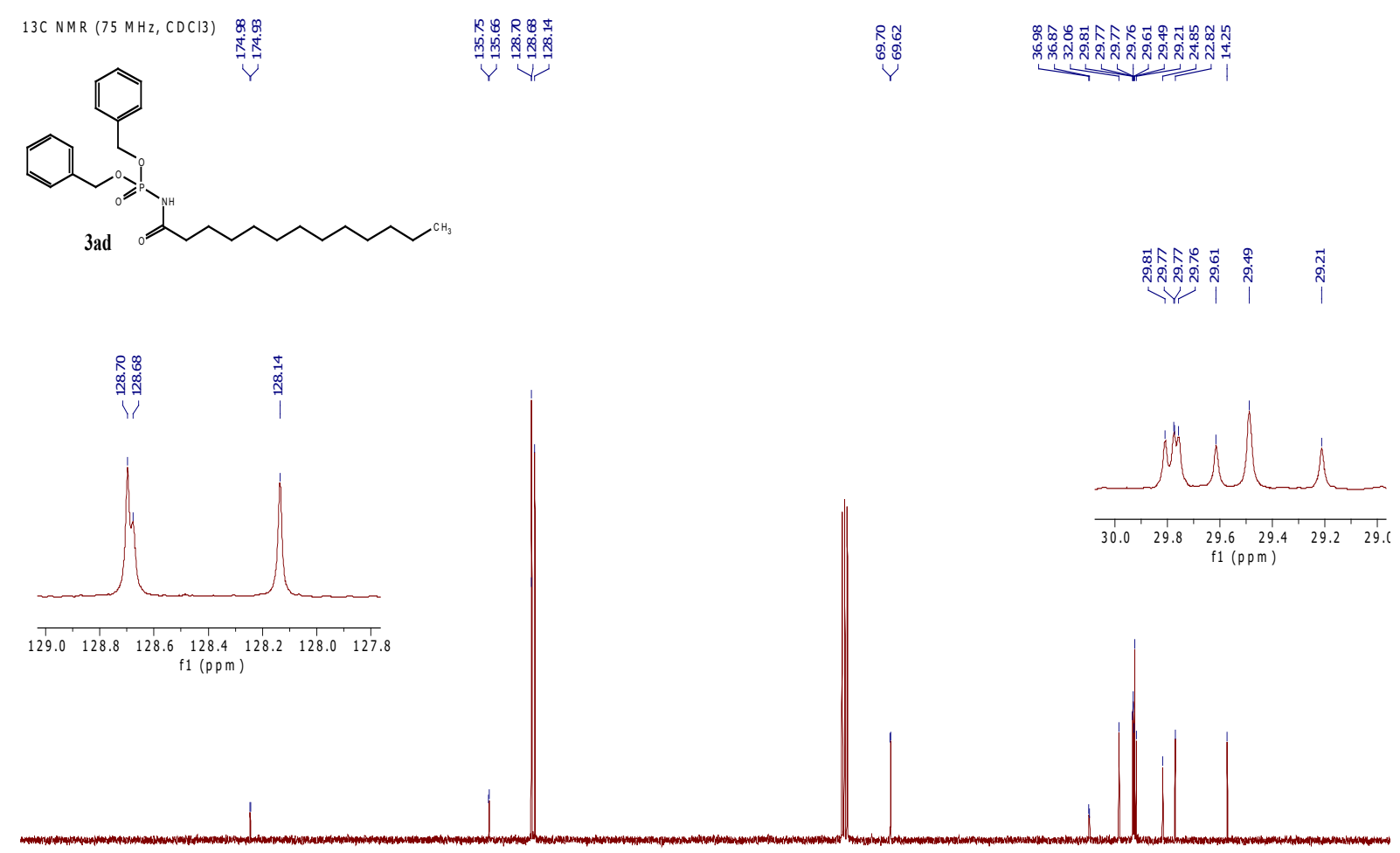

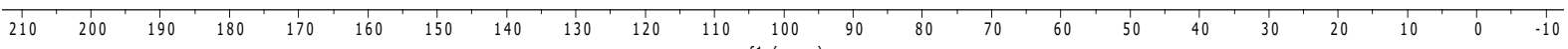



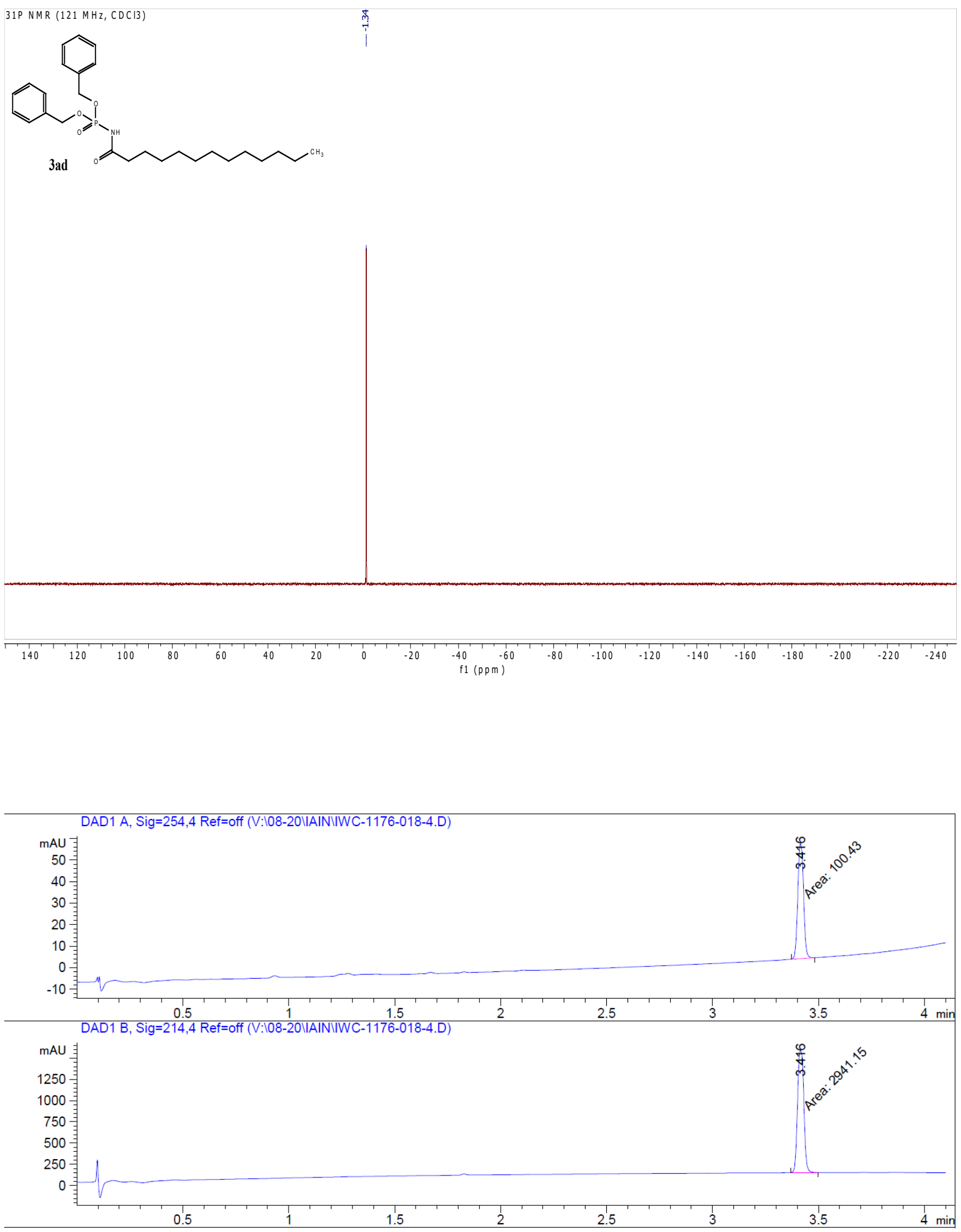
Dibenzyl (2-methyl-2-phenylpropanoyl)phosphoramidate (3ae)
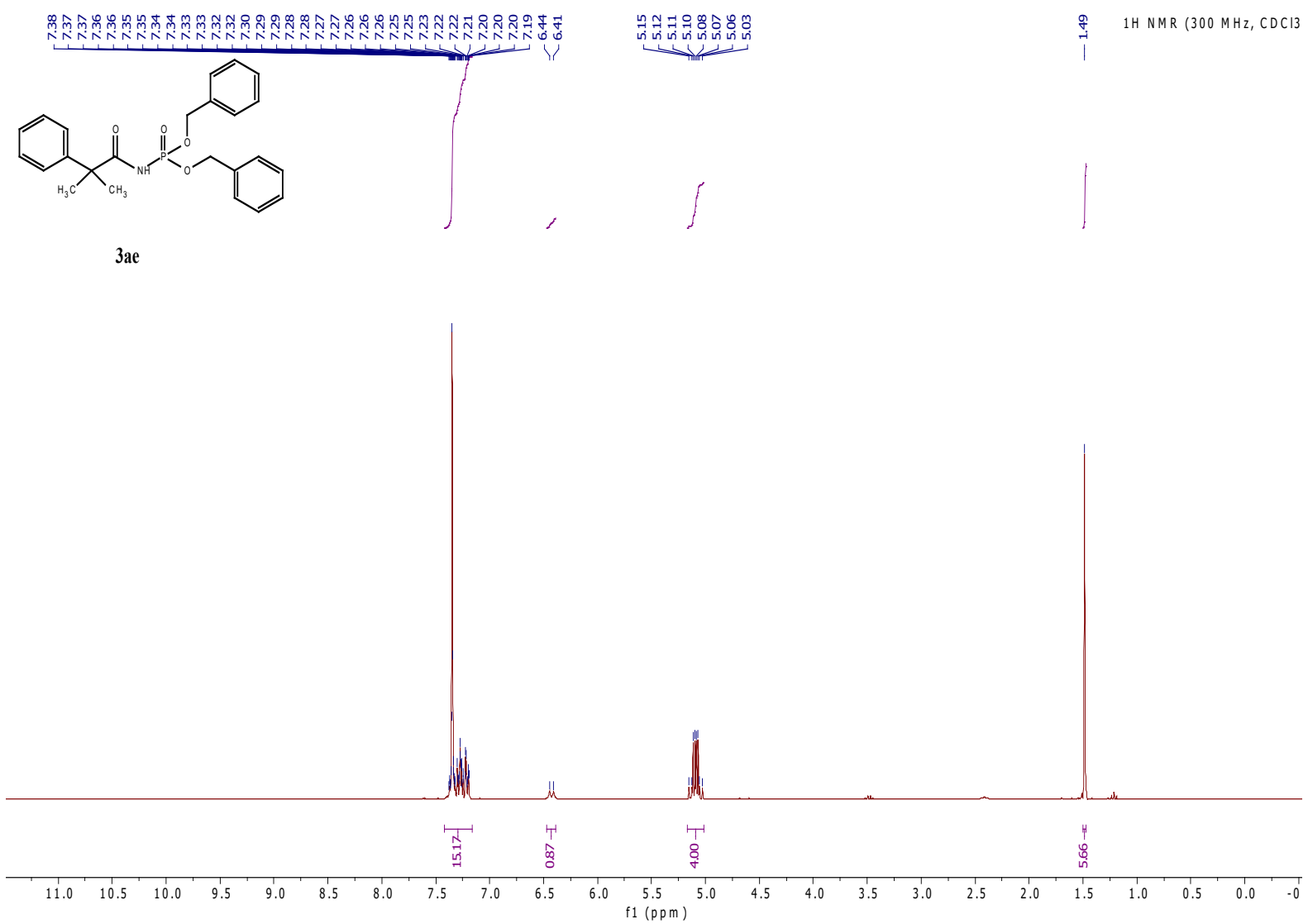

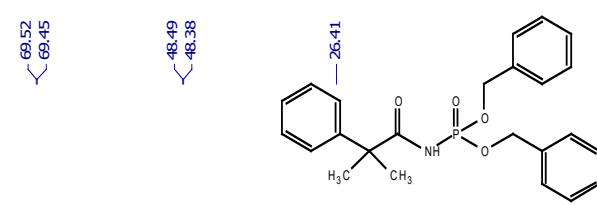

3ae

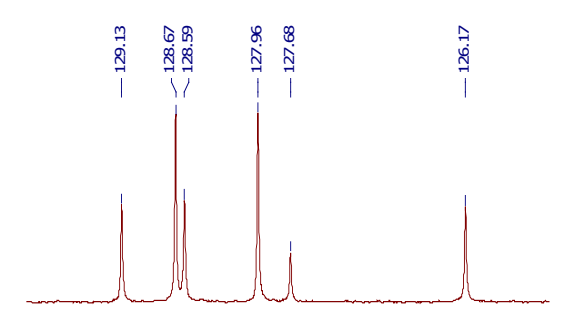

$\begin{array}{llllllllllll}129.5 & 129.0 & 128.5 & 128.0 & 127.5 & 127.0 & 126.5 & 126.0 & 125.5\end{array}$ f1 (ppm)

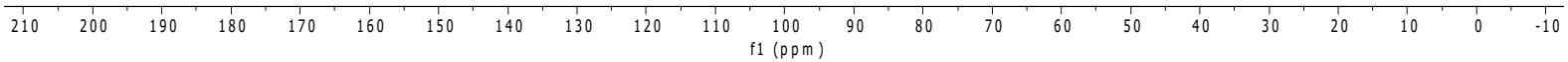



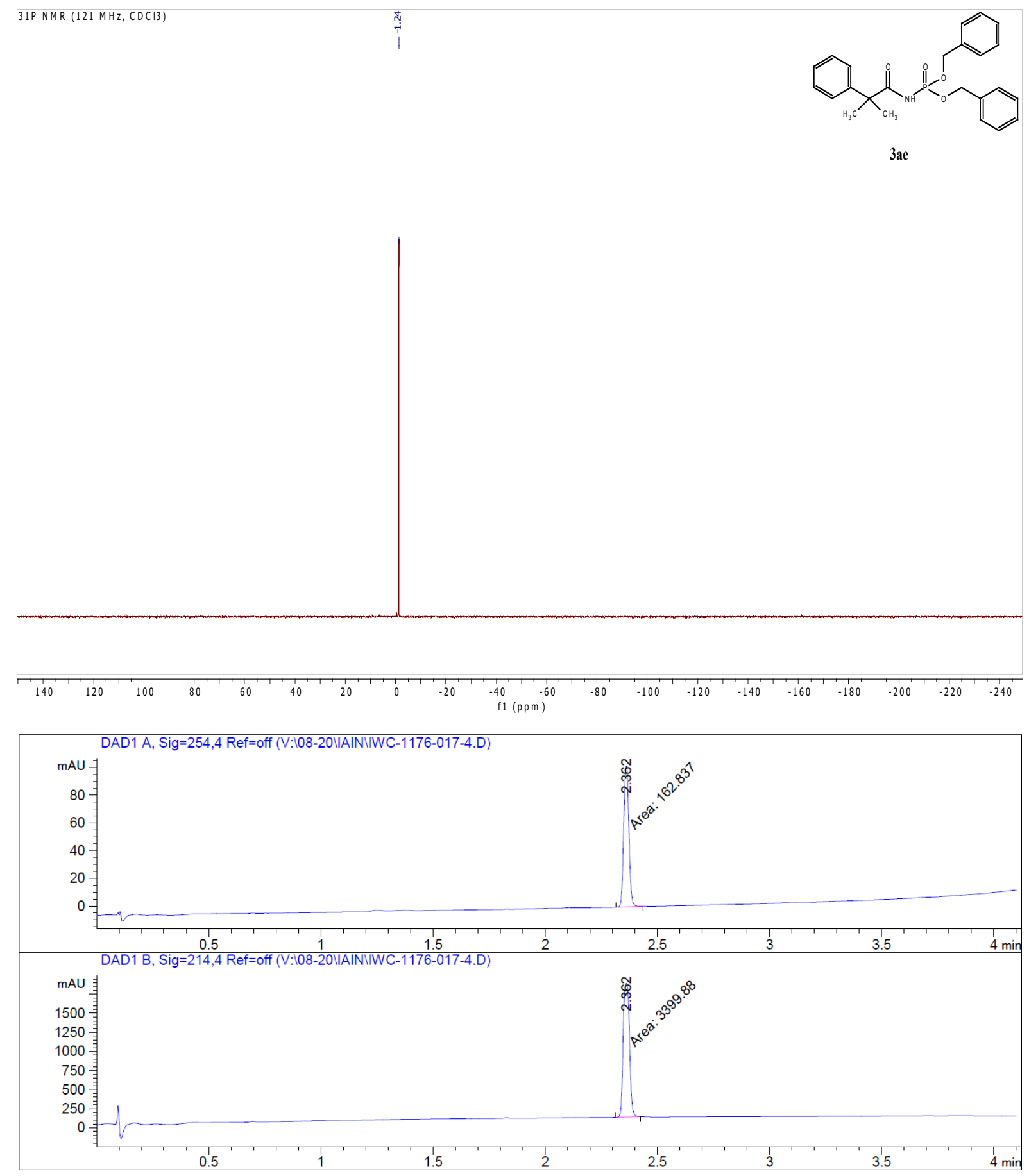
tert-Butyl- $N$-[3-[[(3aR,4R,6R,6aR)-4-(6-aminopurin-9-yl)-2,2-dimethyl-3a,4,6,6atetrahydrofuro[3,4-d][1,3]dioxol-6-yl]methoxyphosphonoyloxy]propyl]carbamate (9)

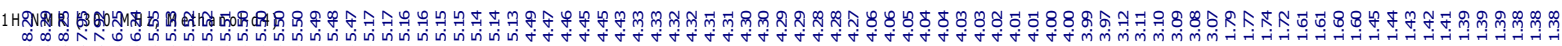

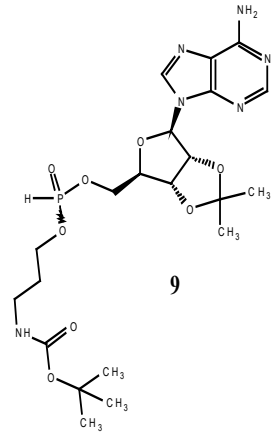

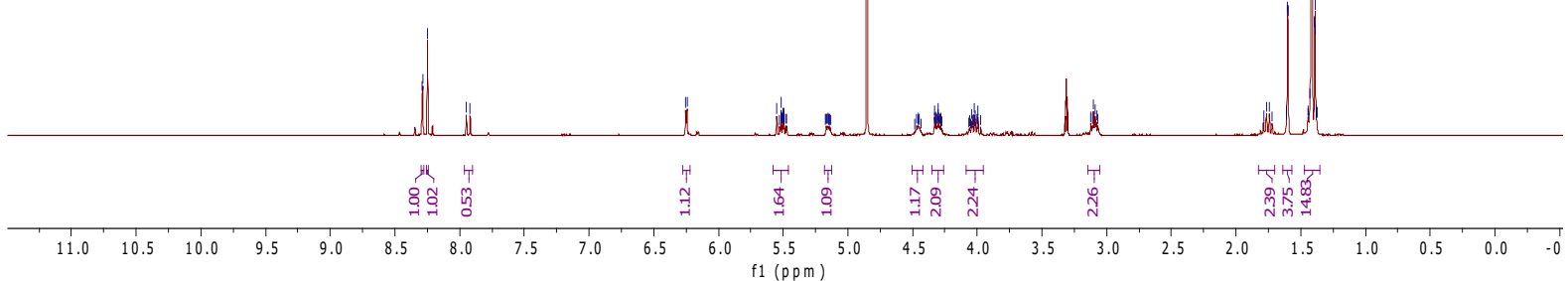




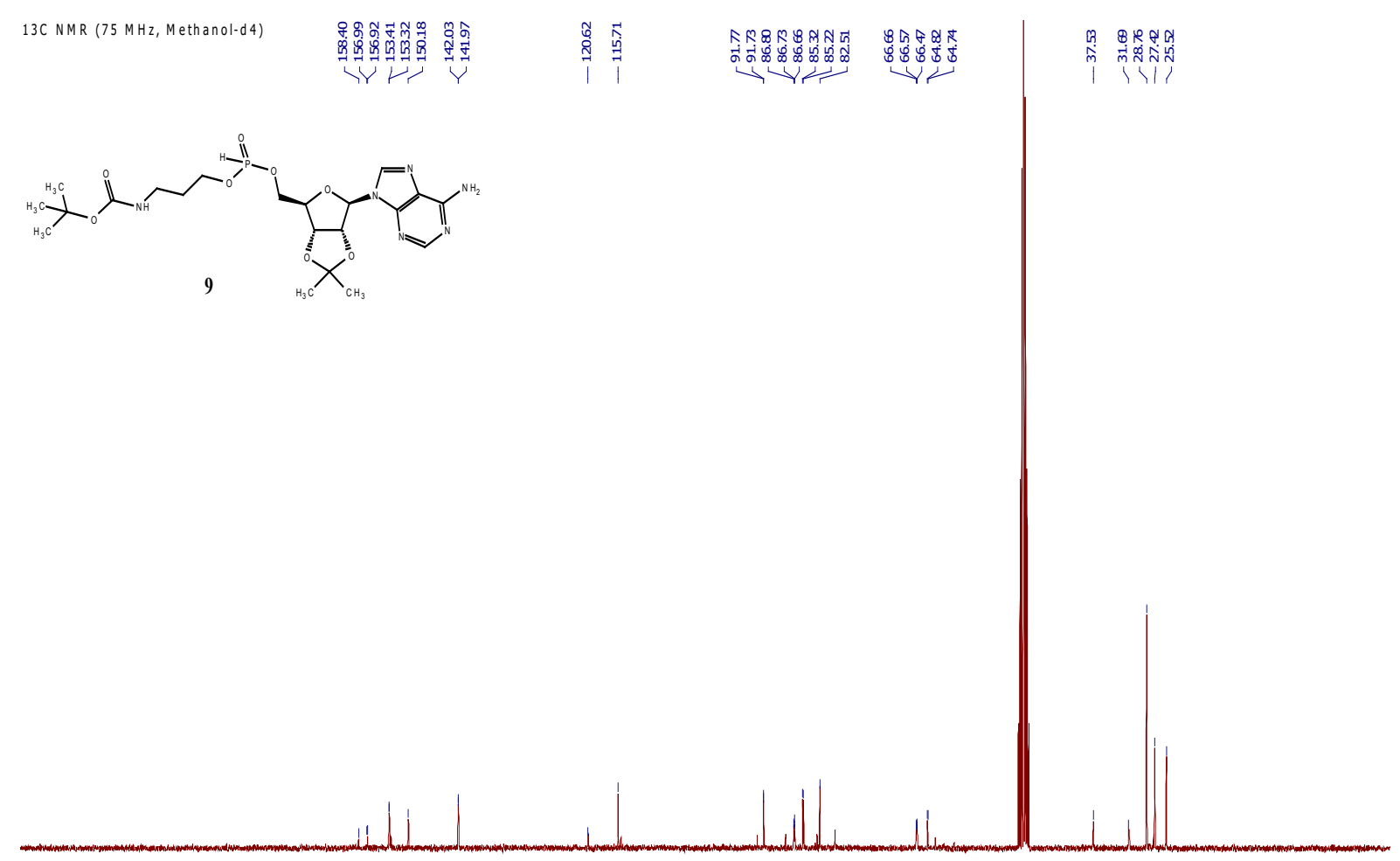

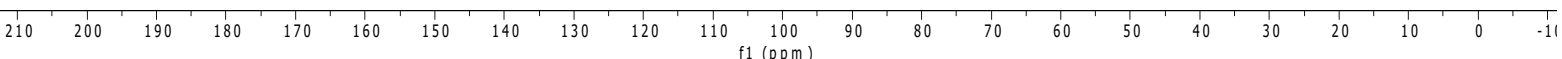

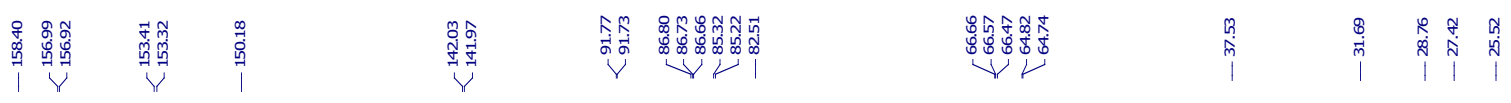

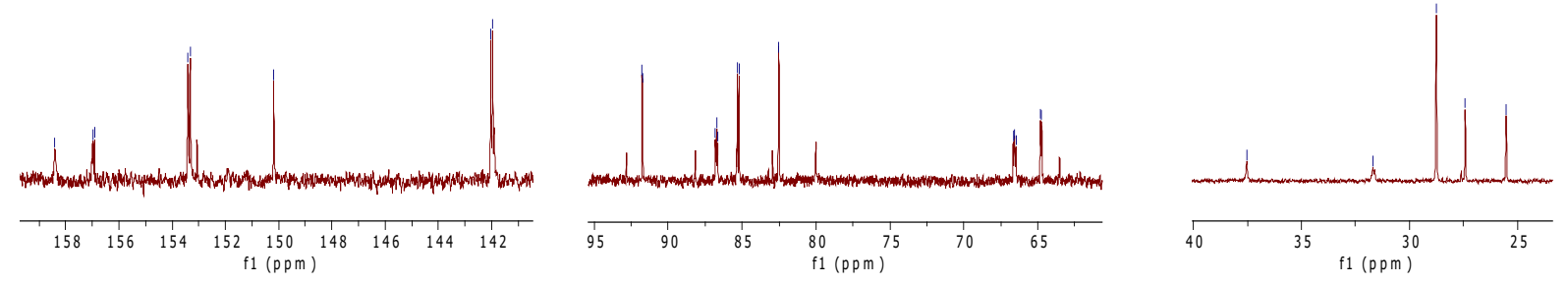



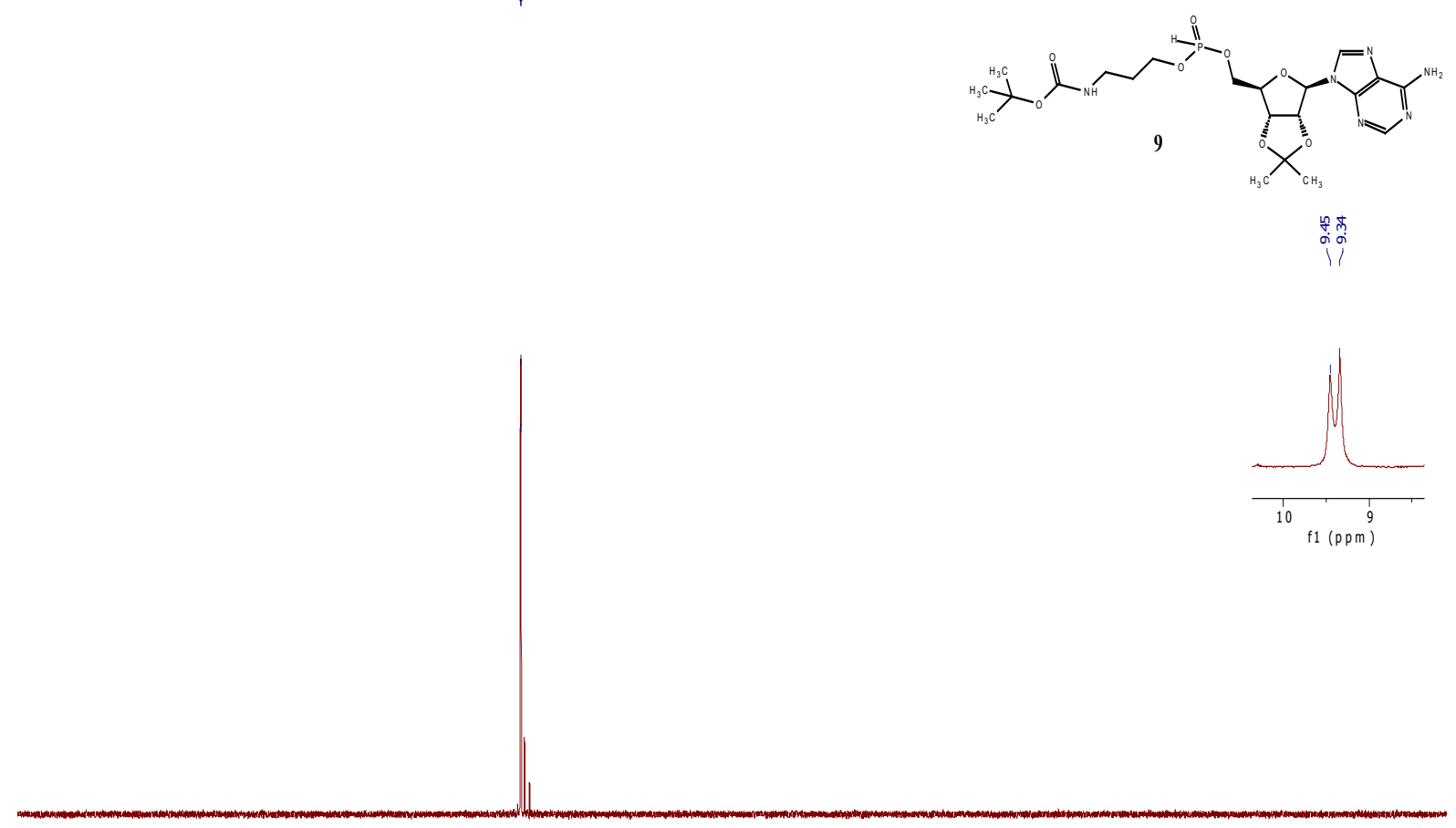

$\begin{array}{cccccccccccccccccccccccccccc}1 & 140 & 120 & 100 & 80 & 60 & 40 & 20 & 0 & -20 & -40 & -60 & -80 & -100 & -120 & -140 & -160 & -180 & -200 & -220 & -240\end{array}$

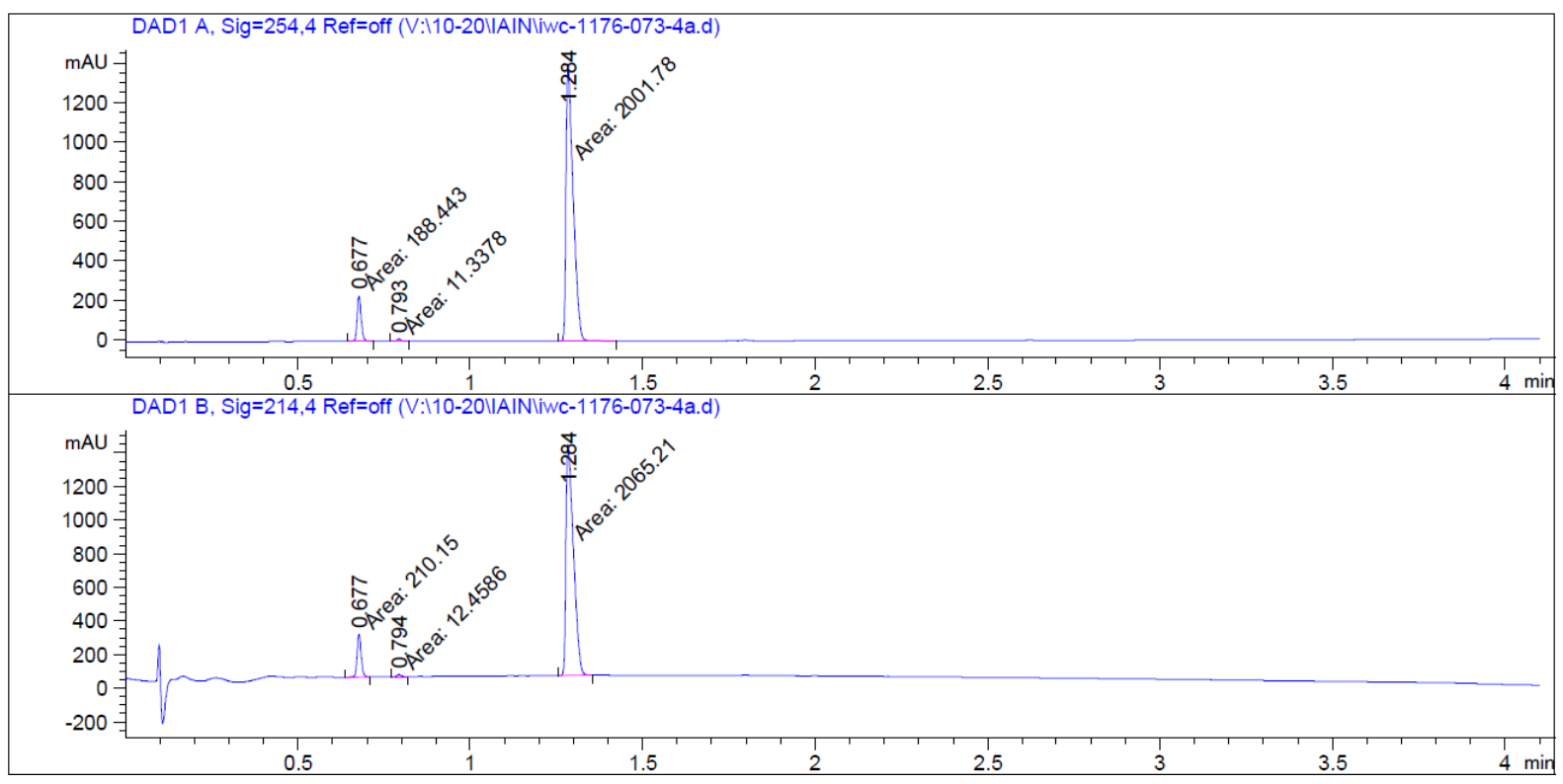


(3S)-tert-Butyl 4-(((((3aR,4R,6R,6aR)-6-(6-amino-9H-purin-9-yl)-2,2-

dimethyltetrahydrofuro[3,4-d][1,3] dioxol-4-yl)methoxy)(3-((tert-

butoxycarbonyl)amino)propoxy)phosphoryl)amino)-3-((tert-butoxycarbonyl)amino)-4oxobutanoate (10)

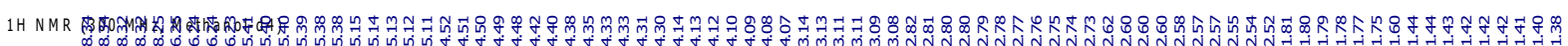

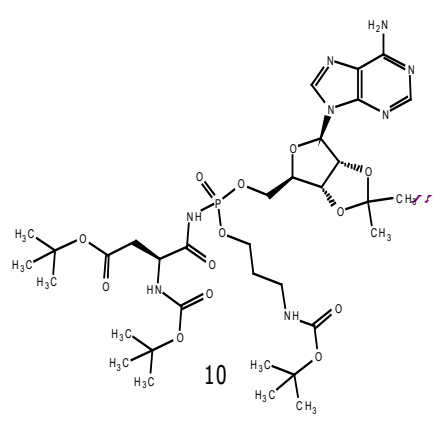

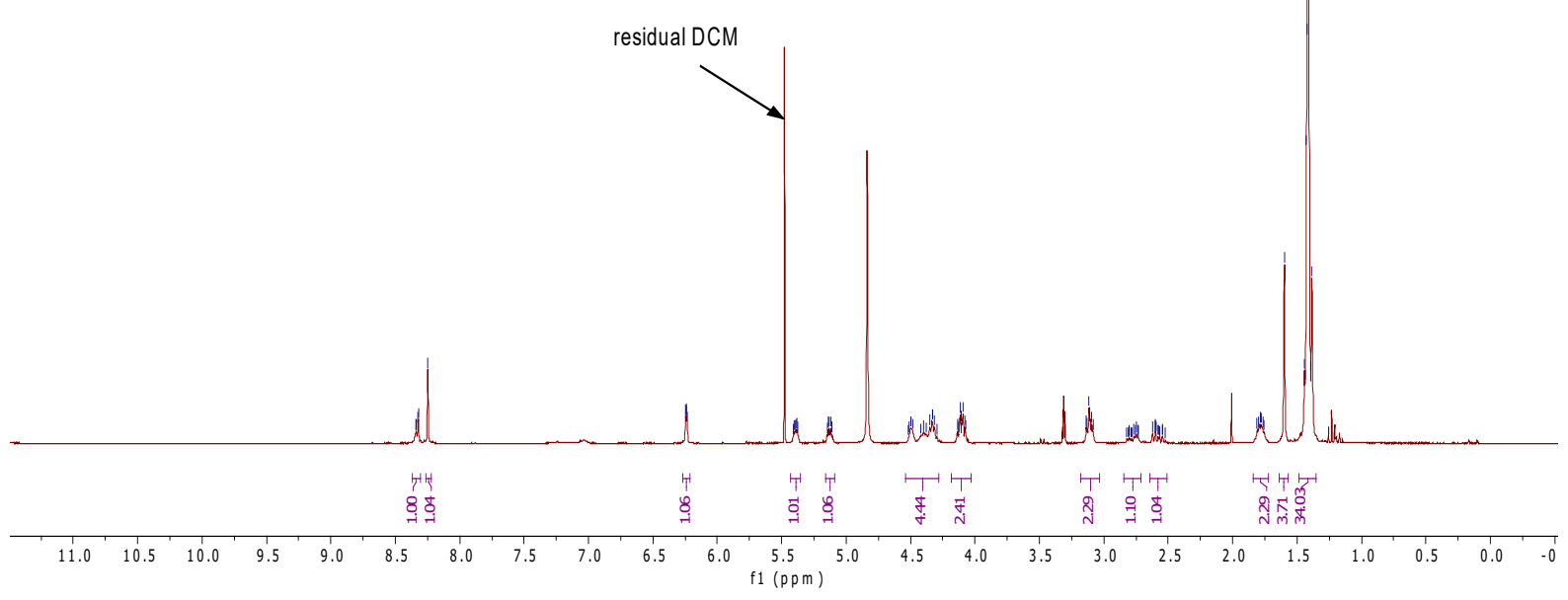



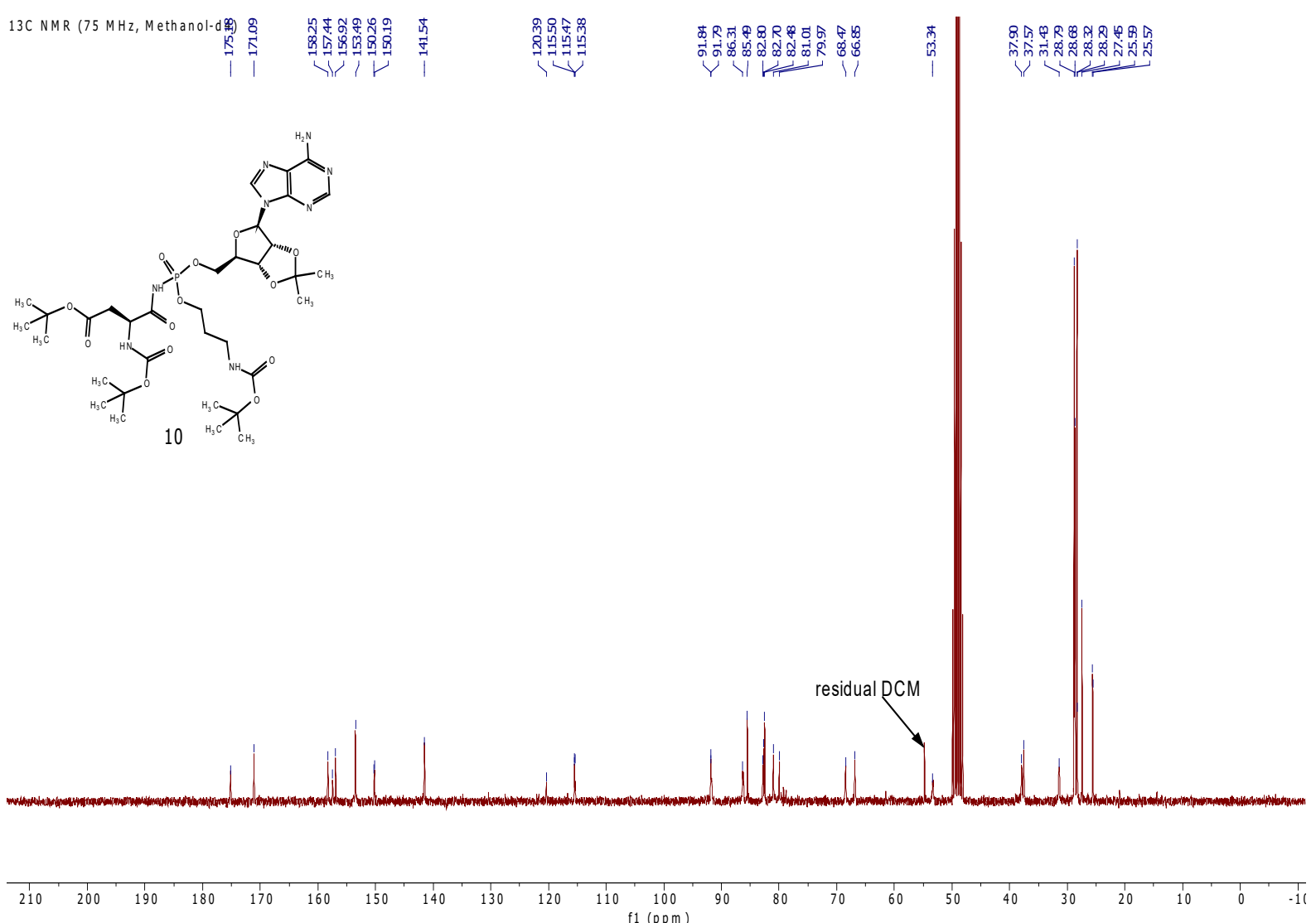

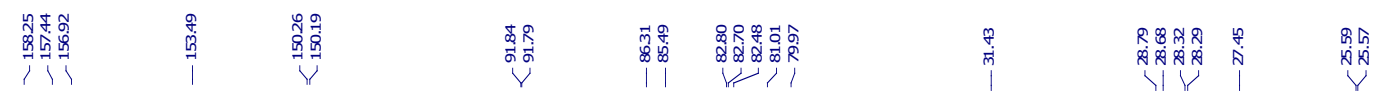
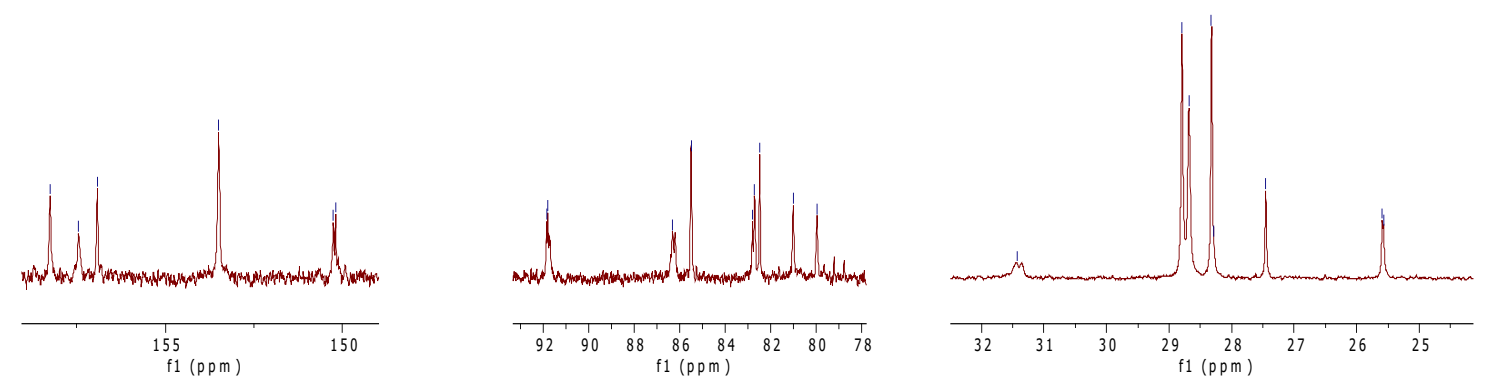
31P NMR (121 MHz, Methanol-d4)
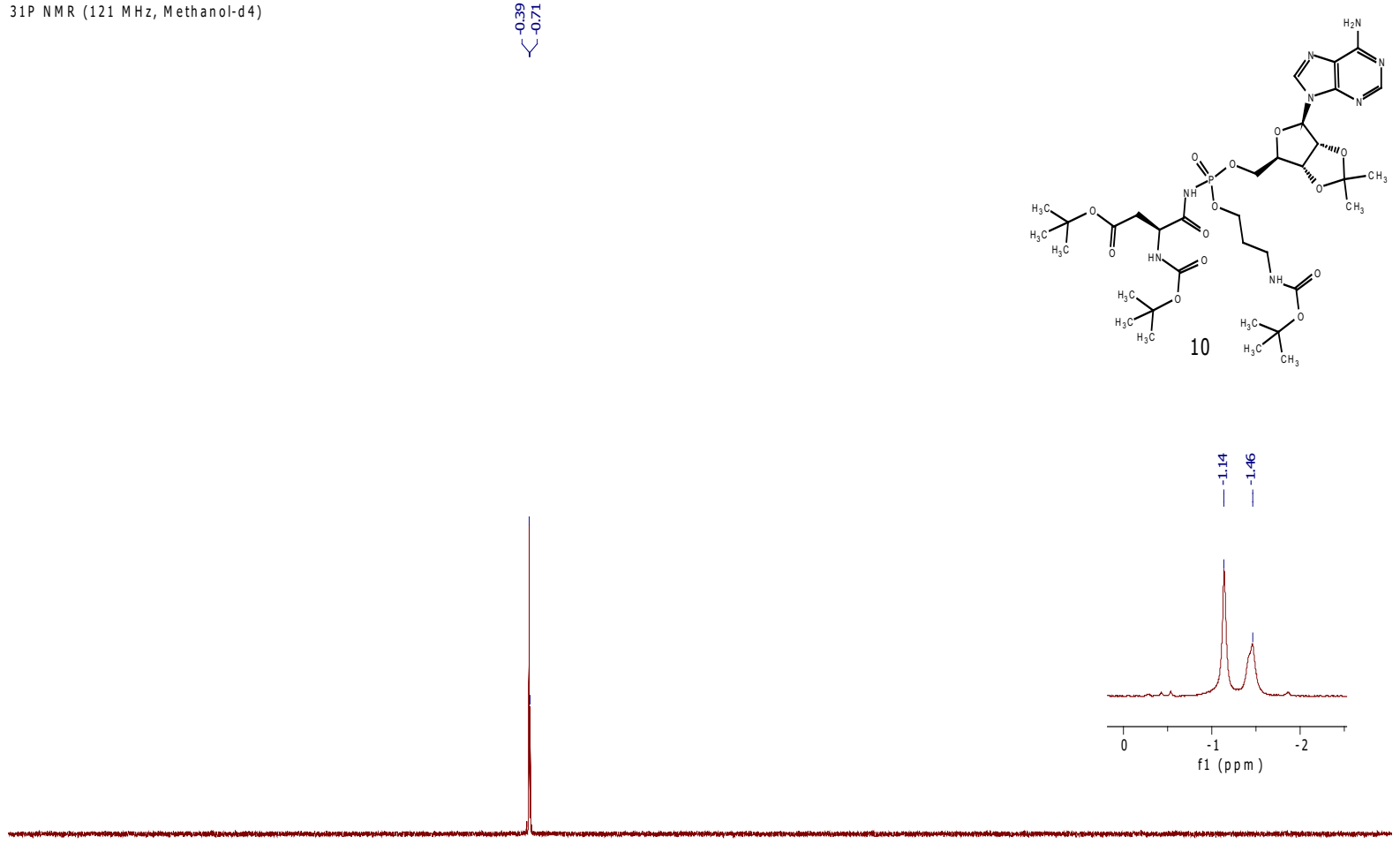

$140 \quad 120$ 
(3S)-3-Amino-4-(((((2R,3S,4R,5R)-5-(6-amino-9H-purin-9-yl)-3,4-

dihydroxytetrahydrofuran-2-yl)methoxy)(3-aminopropoxy)phosphoryl)amino)-4oxobutanoic acid (11)<smiles>CCCCOC(N)(NC(=O)C(C)CC(=O)O)NC1CC(C2CCC3C(C)=CC=NC32)C(C)C1C</smiles>

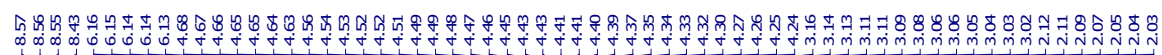
$\checkmark>1$ 工
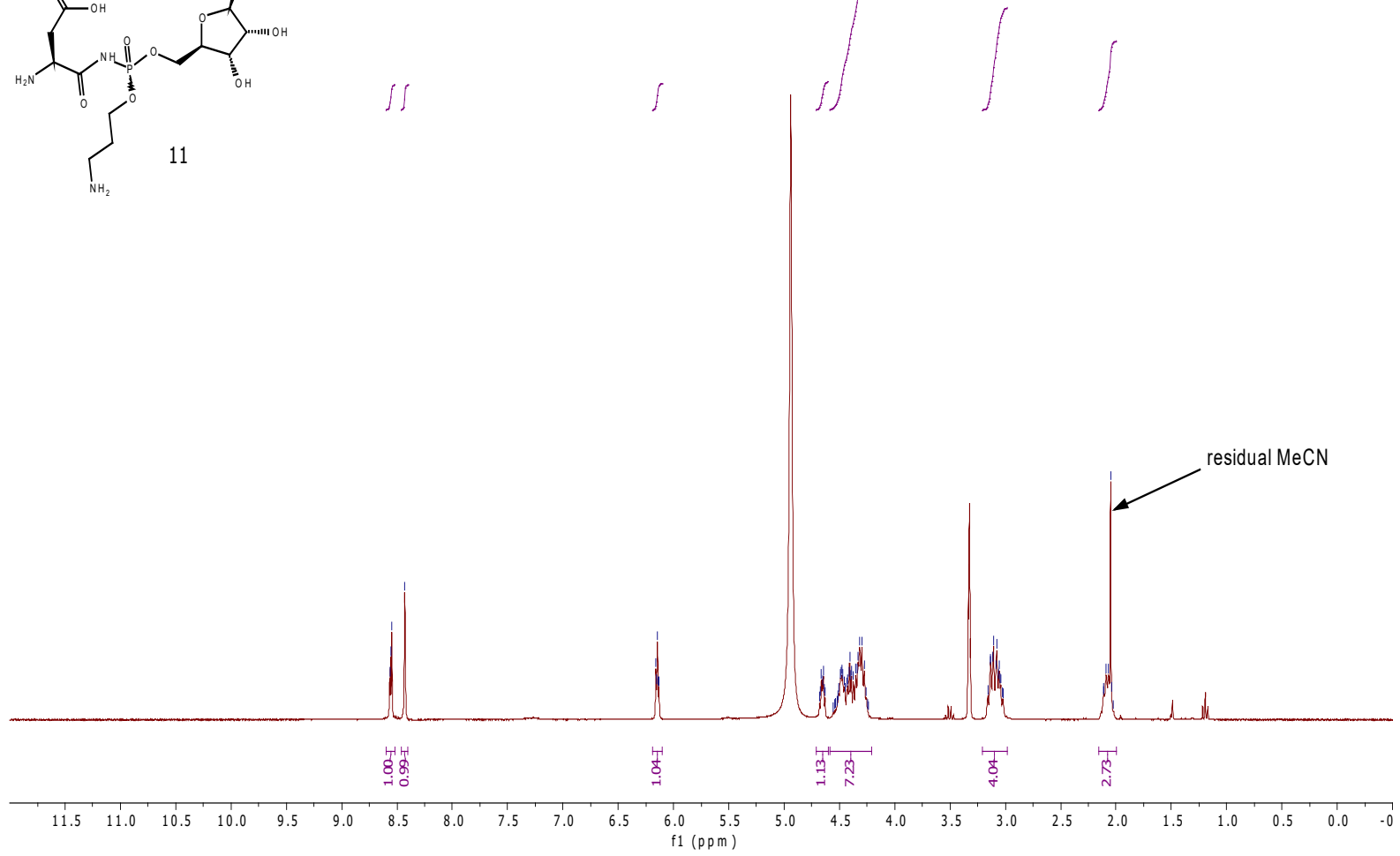

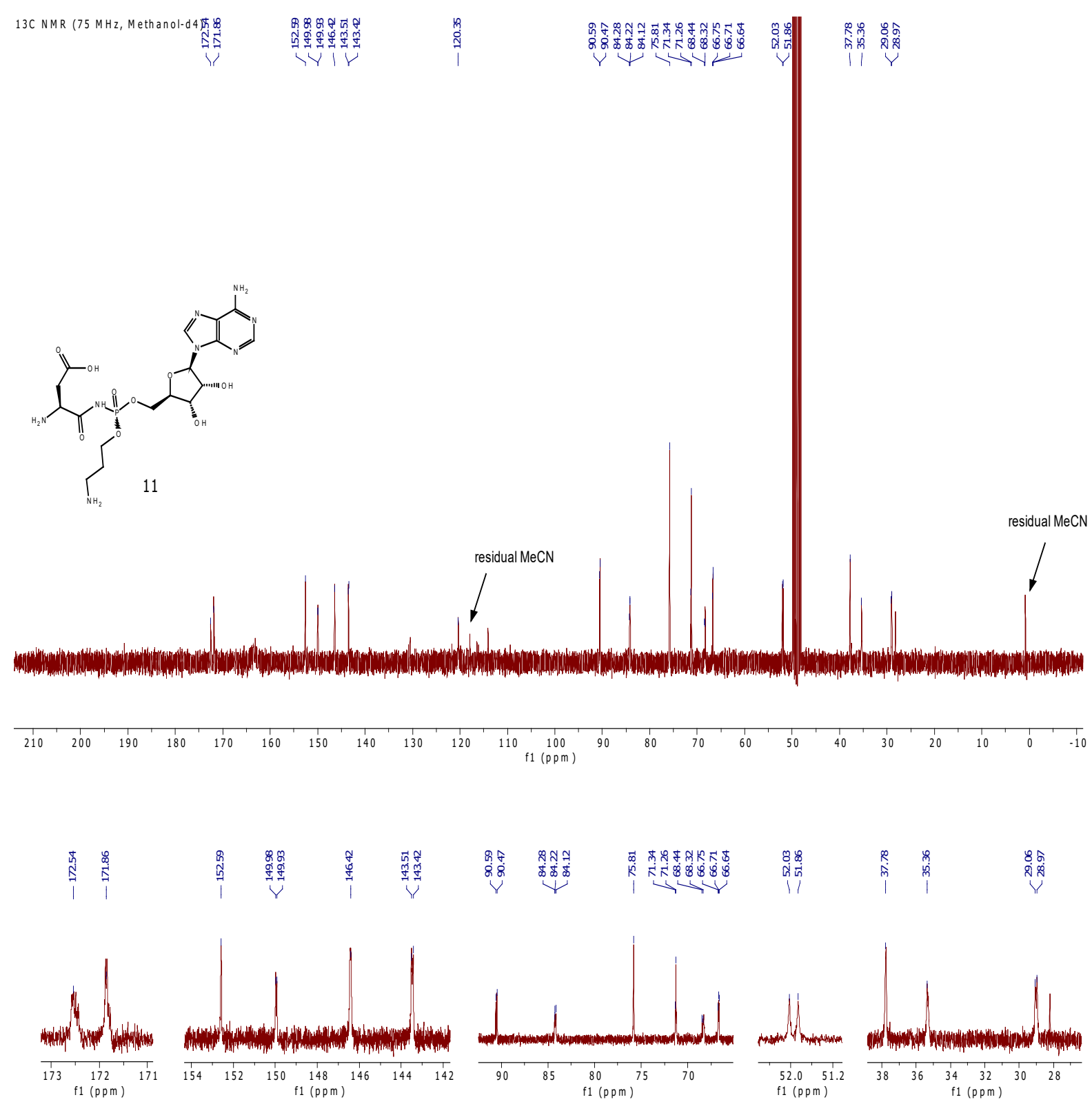
31P NMR (121 MHz, Methanol-d4)

证

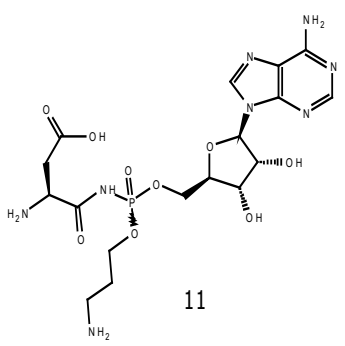

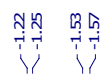
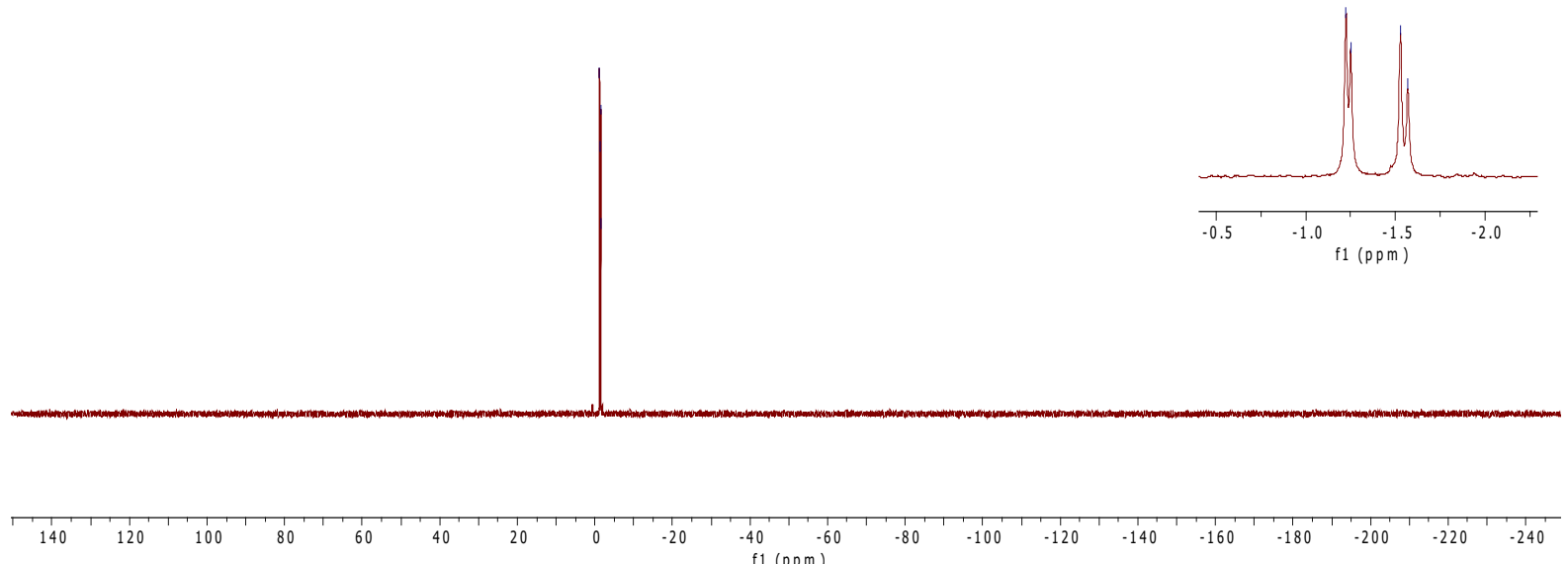

19F NMR (282 MHz, Methanol-d4)

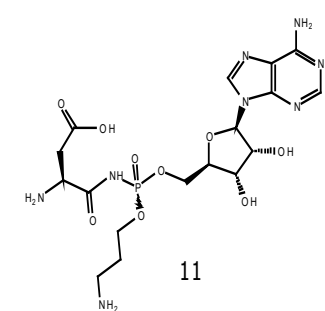

\begin{tabular}{rllllllllllllllllllllllllllllll}
\hline & 10 & 10 & 0 & -10 & -20 & -30 & -40 & -50 & -60 & -70 & -80 & -90 & -100 & -110 & -120 & -130 & -140 & -150 & -160 & -170 & -180 & -190 & -200 & -210 & -22
\end{tabular} 